\title{
Palladium-Catalyzed Asymmetric Trifluoromethylated Allylic Alkylation of Pyrazolones Enabled by $\alpha$-(Trifluoromethyl)alkenyl Acetates
}

Dong Li, Wande Zhang, Shuaibo Zhang, Wuding Sun, Jinfeng Zhao, Baomin Wang, Jingping Qu, Yuhan Zhou*

State Key Laboratory of Fine Chemicals, Department of Pharmaceutical Sciences, School of Chemical Engineering, Dalian University of Technology, 2 Linggong road, Dalian 116024, China. E-mail: zhouyh@dl.cn; zhouyh@dlut.edu.cn

\section{Table of Contents}

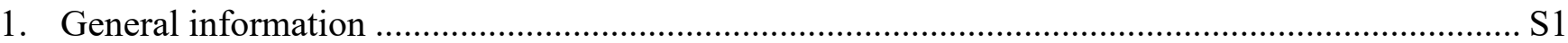

2. Optimization of reaction conditions and control experiments .................................................... S1

3. General procedure for the synthesis of $\alpha$-(trifluoromethyl)alkenyl esters ....................................... S5

4. General procedure for Pd-catalyzed asymmetric trifluoromethylated allylic alkylation S5

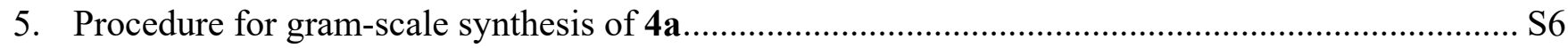

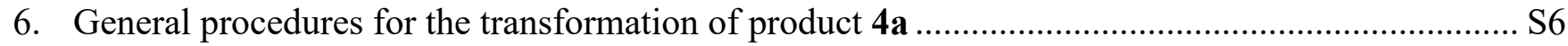

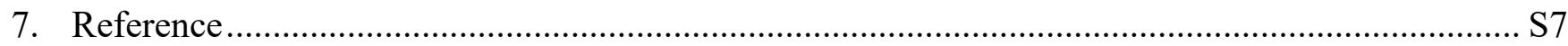

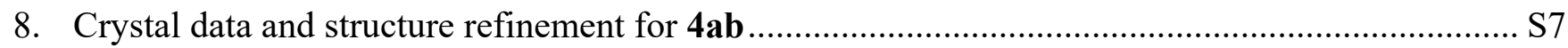

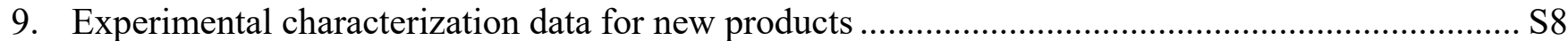

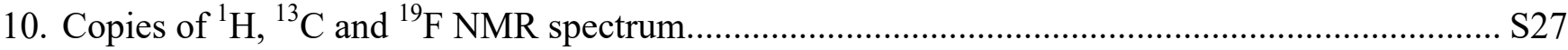

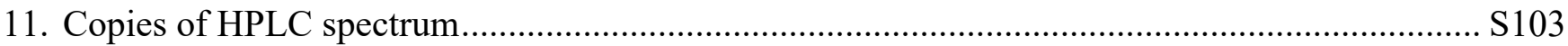




\section{General information}

Unless otherwise noted, all reactions were performed under an argon atmosphere in glassware with magnetic stirring. $\mathrm{NaH}(60 \%$ in mineral oil) was washed with anhydrous $n$-hexane to remove mineral oil prior to use. Other reagents were purchased from commercial sources and used without further purification. All solvents were treated with molecular sieve. Column chromatography was performed on silica gel (200-300 mesh) using petroleum ether/ethyl acetate as eluent. All ${ }^{1} \mathrm{H}$ NMR (400 MHz), ${ }^{13} \mathrm{C}$ NMR (101 MHz) and ${ }^{19} \mathrm{~F}$ NMR (377 MHz or $470 \mathrm{MHz}$ ) were recorded on Bruker AVANCE II-400 or Bruker AVANCE III-500 spectrometers (in $\mathrm{CDCl}_{3}$ with TMS as internal standard). Melting points were recorded on a Novel X-4 spectrometer. HRMS (ESI) were recorded on a Thermo LTQ Orbitrap XL spectrometer. HRMS (EI) were recorded on a Micromass GCT spectrometer. The enantiomeric excess was determined by chiral HPLC with $n$-hexane and $i$-propanol as eluents. Optical rotations were measured on a Rudolph AUTOPOL IV polarimeter. X-ray analysis was performed on a Bruker SMART APEX CCD diffractometer. Pyrazolones were prepared from $\beta$-keto esters according to the literature. ${ }^{1-3}$

\section{Optimization of reaction conditions and control experiments}

Table S1. Optimization of leaving groups. ${ }^{a}$

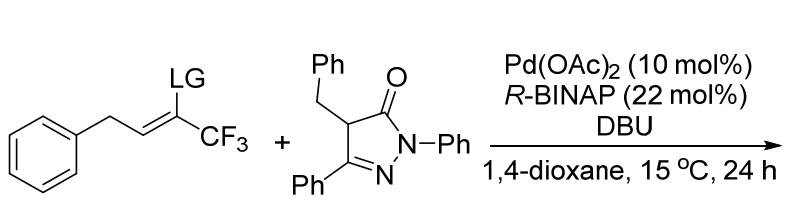

2
$3 a$

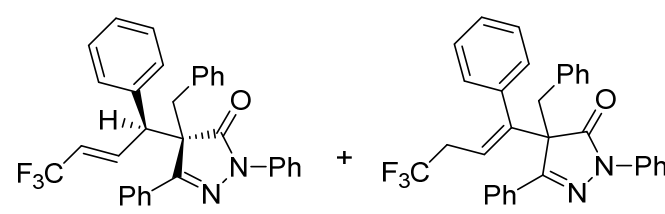

$4 \mathbf{a}$

$5 \mathbf{a}$

\begin{tabular}{cccccc}
\hline Entry & LG & ${\text { Yield of } \mathbf{4 a}(\%)^{b}}^{b}$ & ${\text { Yield of } \mathbf{5 a}(\%)^{b}}^{\mathrm{dr}_{\text {of }} \mathbf{4 a}}{ }^{c}$ & ${\text { ee of } \mathbf{4 a}(\%)^{d}}^{-}$ \\
\hline 1 & OTf & 0 & 0 & -- & - \\
2 & OTs & 0 & 0 & -- & -- \\
3 & OBoc & 63 & 12 & $>20: 1$ & 87 \\
4 & OAc & 44 & $<5$ & $>20: 1$ & 94 \\
5 & OBz & 47 & 8 & $>20: 1$ & 90 \\
\hline
\end{tabular}

${ }^{a}$ Reactions were performed with $2(0.375 \mathrm{mmol}), 3 \mathrm{a}(0.25 \mathrm{mmol}), \mathrm{Pd}(\mathrm{OAc})_{2}(10 \mathrm{~mol} \%), R$-BINAP $(22 \mathrm{~mol} \%)$ and DBU $(0.75 \mathrm{mmol})$ in $3.0 \mathrm{~mL}$ of 1,4 -dioxane at $15{ }^{\circ} \mathrm{C}$ for $24 \mathrm{~h} .{ }^{b}$ Yield of isolated product. ${ }^{c}$ Determined by ${ }^{1} \mathrm{H}$ NMR. ${ }^{d}$ Determined by chiral HPLC. LG $=$ leaving group.

4-Phenyl-1,1,1-trifluorobut-2-en-2-yl trifluoromethanesulfonate was first investigated owing to our continuous study on this molecular but the desired product was failed to detected (Table S1, entry 1). 4-Phenyl-1,1,1-trifluorobut-2-en-2-yl 4-methylbenzenesulfonate was next examed and the desired product was failed to detected too (Table S1, entry 2). We speculate that sulfonyloxy as a substrate for the leaving group is not suitable for this reaction. So next we looked at the 
substrate with carbonyloxy as the leaving group. As shown in Table S1, entries 3-5, substrates with tert-butyl carbonate, acetoxy and benzoyloxy as leaving group all fitted well for this reaction and the desired product was obtained in middle yields amd excellent stereoselectivity. At the same time, 5a was detected as by-product. Specially, the reaction took place smoothly with optimal control of regio-/stereochemistry when acetoxy was used as the leaving group (Table S1, entry 4 , $44 \%$ yield, $>20: 1 \mathrm{dr}, 94 \%$ ee in $24 \mathrm{~h}$ ).

Table S2. Optimization of ligands. ${ }^{a}$

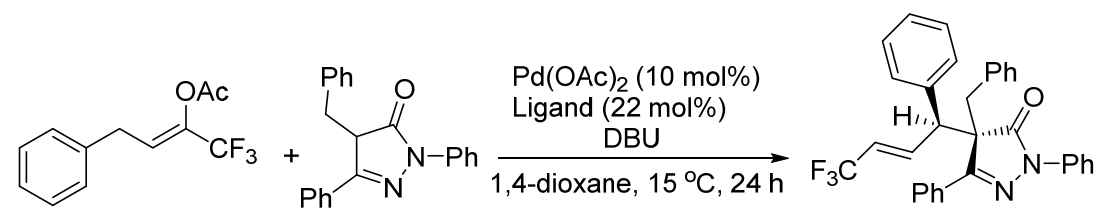

$2 a$

$3 a$

$4 a$

\begin{tabular}{ccccc}
\hline Entry & Ligand & ${\text { Yield of } \mathbf{4 a}(\%)^{b}}^{b}$ & dr of $\mathbf{4 a}^{c}$ & ${\text { ee of } \mathbf{4 a}(\%)^{d}}^{d}$ \\
\hline 1 & L1 & 44 & $>20: 1$ & 94 \\
2 & L2 & 40 & $>20: 1$ & -86 \\
3 & L3 & 30 & $>20: 1$ & 78 \\
4 & L4 & 0 & -- & -- \\
5 & L5 & 0 & -- & -- \\
6 & L6 & 0 & -- & -- \\
7 & L7 & 0 & -- & -- \\
8 & L8 & 0 & -- & -- \\
9 & L9 & 0 & -- & - \\
\hline
\end{tabular}

${ }^{a}$ Reactions were performed with $2 \mathbf{a}(0.375 \mathrm{mmol}), 3 \mathbf{a}(0.25 \mathrm{mmol}), \mathrm{Pd}(\mathrm{OAc})_{2}(10 \mathrm{~mol} \%)$, Ligand $(22 \mathrm{~mol} \%)$ and DBU $(0.75 \mathrm{mmol})$ in $3.0 \mathrm{~mL}$ of 1,4 -dioxane at $15{ }^{\circ} \mathrm{C}$ for $24 \mathrm{~h} .{ }^{b}$ Yield of isolated product. ${ }^{c}$ Determined by ${ }^{1} \mathrm{H}$ NMR. ${ }^{d}$ Determined by chiral HPLC.

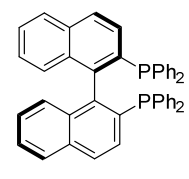
$R$-BINAP L1

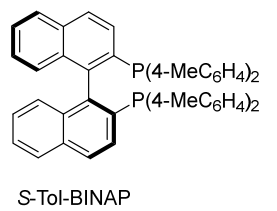

L2

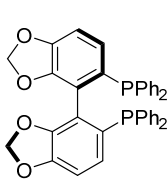

L3

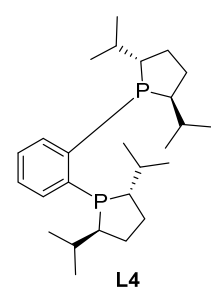

L4

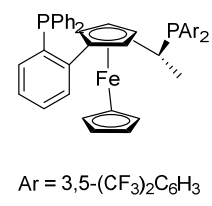

L5

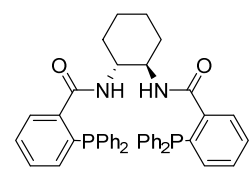

L6

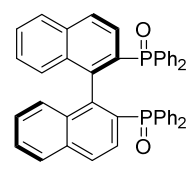

L7

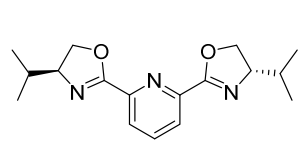

L8

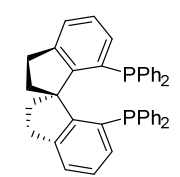

L9

As shown in Table S2, several chiral ligands were investigated and the reaction took place when L1 ( $R$-BINAP), L2 ( $S$-Tol-BINAP), and L3 were used. The enantioselectives of 4a was decreased when switching $\mathrm{L} 1$ to $\mathrm{L} 2$ or L3 (-86\% ee and $78 \%$ ee respectively). Ligands L4-L9 are 
not suitable for this reaction because the reaction did not occur when they were used.

Table S3. Optimization of catalysts and bases. ${ }^{a}$

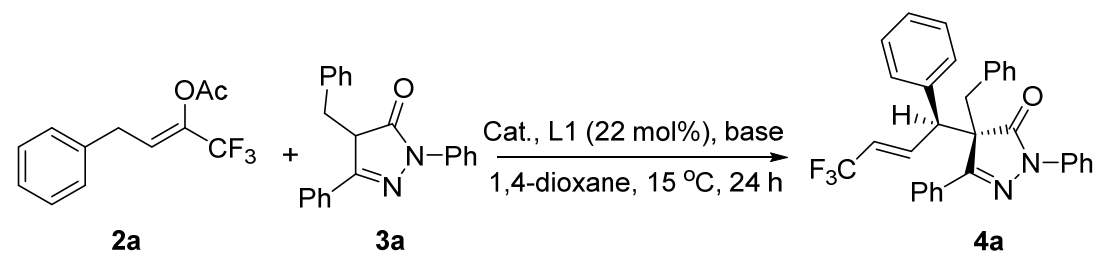

\begin{tabular}{|c|c|c|c|c|c|}
\hline Entry & Cat. ( $\mathrm{mol} \%)$ & Base & Yield of $4 a(\%)^{b}$ & $\mathrm{dr}$ of $\mathbf{4 a ^ { c }}$ & ee of $4 \mathbf{a}(\%)^{d}$ \\
\hline 1 & $\mathrm{Pd}(\mathrm{OAc})_{2}(10 \mathrm{~mol} \%)$ & DBU & 44 & $>20: 1$ & 94 \\
\hline 2 & $\mathrm{PdCl}_{2}(10 \mathrm{~mol} \%)$ & DBU & 0 & -- & -- \\
\hline 3 & $\mathrm{PdCl}_{2}\left(\mathrm{PPh}_{3}\right)_{2}(10 \mathrm{~mol} \%)$ & DBU & 0 & -- & -- \\
\hline 4 & $\mathrm{Pd}_{2}(\mathrm{dba})_{3}(5 \mathrm{~mol} \%)$ & DBU & 0 & -- & -- \\
\hline 5 & {$[\mathrm{Pd}(\text { allyl }) \mathrm{Cl}]_{2}(5 \mathrm{~mol} \%)$} & DBU & 52 & $>20: 1$ & 91 \\
\hline 6 & $\mathrm{Pd}(\mathrm{OAc})_{2}(10 \mathrm{~mol} \%)$ & TBD & 0 & -- & -- \\
\hline 7 & $\mathrm{Pd}(\mathrm{OAc})_{2}(10 \mathrm{~mol} \%)$ & $\mathrm{Et}_{3} \mathrm{~N}$ & 0 & -- & -- \\
\hline 8 & $\mathrm{Pd}(\mathrm{OAc})_{2}(10 \mathrm{~mol} \%)$ & TMG & 0 & -- & -- \\
\hline 9 & $\mathrm{Pd}(\mathrm{OAc})_{2}(10 \mathrm{~mol} \%)$ & DABCO & 0 & -- & -- \\
\hline 10 & $\mathrm{Pd}(\mathrm{OAc})_{2}(10 \mathrm{~mol} \%)$ & DMAP & 0 & -- & -- \\
\hline 11 & $\mathrm{Pd}(\mathrm{OAc})_{2}(10 \mathrm{~mol} \%)$ & DIPEA & 0 & -- & -- \\
\hline 12 & $\mathrm{Pd}(\mathrm{OAc})_{2}(10 \mathrm{~mol} \%)$ & pyiridine & 0 & -- & -- \\
\hline 13 & $\mathrm{Pd}(\mathrm{OAc})_{2}(10 \mathrm{~mol} \%)$ & piperidine & 0 & -- & -- \\
\hline
\end{tabular}

${ }^{a}$ Reactions were performed with $2 \mathrm{a}(0.375 \mathrm{mmol}), \mathbf{3 a}(0.25 \mathrm{mmol})$, Cat., $R$-BINAP $(22 \mathrm{~mol} \%)$ and base $(0.75$ mmol) in $3.0 \mathrm{~mL}$ of 1,4 -dioxane at $15{ }^{\circ} \mathrm{C}$ for $24 \mathrm{~h}$. ${ }^{b}$ Yield of isolated product. ${ }^{c}$ Determined by ${ }^{1} \mathrm{H}$ NMR.

${ }^{d}$ Determined by chiral HPLC.

Then the palladium-catalysts were explored (Table $\mathrm{S} 3$, entries 1-5) and $\mathrm{Pd}(\mathrm{OAc})_{2}$ was proved to be the best catalyst with slightly higher enantioselectivity than $\left[\mathrm{Pd}(\text { ally)Cl}]_{2}\right.$ (Table S1, entry 1 and entry 5, 94\% ee and $91 \%$ ee respectively). The reaction did not occur when $\mathrm{PdCl}_{2}, \mathrm{Pd}_{2}(\mathrm{dba})_{3}$ and $\mathrm{PdCl}_{2}\left(\mathrm{PPh}_{3}\right)_{2}$ were used.

The screening of bases showed that the reaction did not occur when switching DBU to other organobases (Table S3, entries 6-13), indicating that DBU was more efficient for the double-bond migration of $\alpha$-(trifluoromethyl)alkenyl acetates. So experiments were next performed to detect whether the double bond of $\alpha$-(trifluoromethyl)alkenyl acetates is isomerized in the presence of different organobases. As shown in Table S4, DBU is the most suitable base for the double-bond migration of $\alpha$-(trifluoromethyl)alkenyl acetates. 
Table S4. Double-bond migration of $2 \mathbf{a}$ with different bases. ${ }^{a}$

\begin{tabular}{|c|c|c|c|}
\hline Entry & Base & Conversion of $2 \mathbf{a}(\%)^{b}$ & Yield of $\mathbf{2} \mathbf{a}^{\prime}(\%)^{b}$ \\
\hline 1 & DBU & 100 & 100 \\
\hline 2 & TBD & 0 & 0 \\
\hline 3 & $\mathrm{Et}_{3} \mathrm{~N}$ & 8 & 8 \\
\hline 4 & TMG & 0 & 0 \\
\hline 5 & DABCO & 17 & 17 \\
\hline 6 & DMAP & 0 & 0 \\
\hline 7 & DIPEA & 0 & 0 \\
\hline 8 & pyiridine & 0 & 0 \\
\hline 9 & piperidine & 0 & 0 \\
\hline
\end{tabular}

${ }^{a}$ Reactions were performed with $\mathbf{2 a}(0.25 \mathrm{mmol})$, base $(0.75 \mathrm{mmol})$ in $2.0 \mathrm{~mL}$ of 1 ,4-dioxane at $15{ }^{\circ} \mathrm{C}$ for $24 \mathrm{~h}$.

${ }^{b}$ Yield and conversion were determined by ${ }^{1} \mathrm{H}$ NMR.

Table S5. Optimization of equivalents of ligand, DBU and 2a. ${ }^{a}$

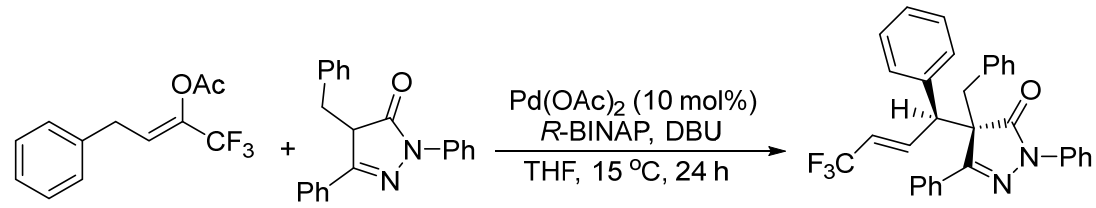

$2 \mathbf{a}$

$3 \mathbf{a}$

$4 a$

\begin{tabular}{ccccccc}
\hline Entry & $\mathbf{2 a}$ & $R$-BINAP & DBU & ${\text { Yield of } \mathbf{4 a}(\%)^{b}}^{b}$ & $\operatorname{dr}^{\text {of }} \mathbf{4 a ^ { c }}$ & ee of $\mathbf{4 a ~ ( \% ) ~}^{d}$ \\
\hline 1 & 1.5 eq. & $22 \mathrm{~mol} \%$ & 3.0 eq. & 40 & $>20: 1$ & 96 \\
2 & 1.5 eq. & $16 \mathrm{~mol} \%$ & 3.0 eq. & 46 & $>20: 1$ & 95 \\
3 & 1.5 eq. & $12 \mathrm{~mol} \%$ & 3.0 eq. & 51 & $>20: 1$ & 96 \\
4 & 1.5 eq. & $10 \mathrm{~mol} \%$ & 3.0 eq. & 49 & $>20: 1$ & 93 \\
5 & 1.2 eq. & $12 \mathrm{~mol} \%$ & 3.0 eq. & 50 & $>20: 1$ & 94 \\
6 & 1.5 eq. & $12 \mathrm{~mol} \%$ & 2.0 eq. & 50 & $>20: 1$ & 95 \\
7 & 1.5 eq. & $12 \mathrm{~mol} \%$ & 1.5 eq. & 52 & $>20: 1$ & 96 \\
8 & 1.5 eq. & $12 \mathrm{~mol} \%$ & 1.2 eq. & 44 & $>20: 1$ & 96 \\
$9^{e}$ & 1.5 eq. & $12 \mathrm{~mol} \%$ & 1.5 eq. & 80 & $>20: 1$ & 96 \\
\hline
\end{tabular}


${ }^{a}$ Reactions were performed with 2a, 3a $(0.25 \mathrm{mmol}), \mathrm{Pd}(\mathrm{OAc})_{2}(10 \mathrm{~mol} \%), R$-BINAP and DBU in $3.0 \mathrm{~mL}$ of THF at $15{ }^{\circ} \mathrm{C}$ for $24 \mathrm{~h}^{b}{ }^{b}$ Yield of isolated product 4a. ${ }^{c}$ Determined by ${ }^{1} \mathrm{H}$ NMR. ${ }^{d}$ Determined by chiral HPLC. e $52 \mathrm{~h}$.

The equivalents of ligand, DBU and 2a were next optimized. As shown in Table S5, entires 1-3, both the yield and stereoselectivity of $\mathbf{4 a}$ were maintained when the equivalent of $R$-BINAP was gradually reduced from $22 \mathrm{~mol} \%$ to $12 \mathrm{~mol} \%$. However, the stereoselectivity of $\mathbf{4 a}$ was decreased when the amount of $R$-BINAP was reduced to $10 \mathrm{~mol} \%$. The stereoselectivity of $\mathbf{4 a}$ is slightly lower when the amount of $\mathbf{2} \mathbf{a}$ was reduced to 1.2 equivalents (Table S5, entry 5, 94\% ee). Both the yield and stereoselectivity of $\mathbf{4 a}$ were maintained when the equivalent of DBU was reduced form 3.0 equivalent to 1.5 equivalent but the yield of $\mathbf{4 a}$ was decreased when 1.2 equivalent of DBU was used (Table S5, entires 3, 6-8). At last, the yield of $4 \mathbf{a}$ was increased to $80 \%$ with maintained stereoselectivity when the reaction time was exented to $52 \mathrm{~h}$.

Scheme S1. The experiment of double-bond migration in $\mathbf{2 p .}$

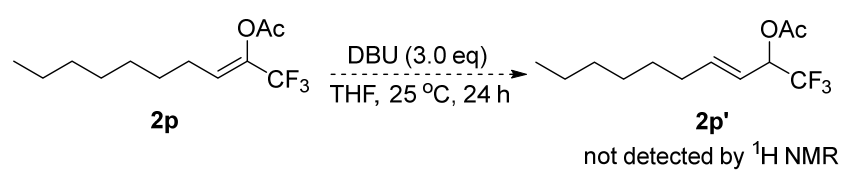

\section{General procedure for the synthesis of $\alpha$-(trifluoromethyl)alkenyl esters}

$\alpha$-(Trifluoromethyl)alkenyl esters were preparated following our previous works on synthesizing of $\alpha$-trifluoromethyl alkenyl triflates. ${ }^{4}$

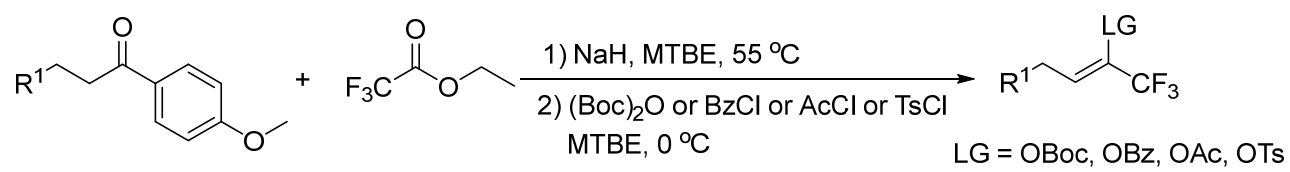

To a suspension of $\mathrm{NaH}(480 \mathrm{mg}, 20.0 \mathrm{mmol})$ in MTBE $(50 \mathrm{~mL})$ was added ethyl trifluoroacetate $(2.4 \mathrm{~mL}, 20.0 \mathrm{mmol})$ at room temperature under argon atmosphere. After about $1 \mathrm{~min}$ of stirring, enolizable ketone $(10.0 \mathrm{mmol})$ was added, and the mixture was refluxed for 6-12 $\mathrm{h}$. After the reaction was complete (monitored by TLC or GC analyses), the reaction solution was cooled to $0{ }^{\circ} \mathrm{C}$. Di-tert-butyl dicarbonate $\left((\mathrm{Boc})_{2} \mathrm{O}, 20.0 \mathrm{mmol}\right)$ or benzoyl chloride $(\mathrm{BzCl}, 20.0 \mathrm{mmol})$ or acetyl chloride ( $\mathrm{AcCl}, 20.0 \mathrm{mmol}$ ) or tosyl chloride ( $\mathrm{TsCl}, 20.0 \mathrm{mmol}$ ) was added slowly into the reaction mixture. After the reaction was complete (5-15 min, monitored by TLC or GC analyses), the reaction was quenched with ice-water. The aqueous layer was separated and extracted with ethyl acetate. The combined organic extracts were washed with brine, dried over $\mathrm{Na}_{2} \mathrm{SO}_{4}$, and the solvent was removed under reduced pressure. The crude product was purified by column chromatography on silica gel to afford the products.

\section{General procedure for Pd-catalyzed asymmetric trifluoromethylated allylic alkylation}




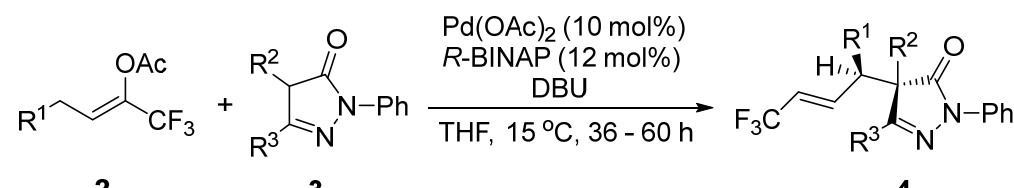

Dissolving the $\mathrm{Pd}(\mathrm{OAc})_{2}(11.2 \mathrm{mg}, 0.05 \mathrm{mmol}, 10 \mathrm{~mol} \%)$ and $R$-BINAP (37.4 mg, $0.06 \mathrm{mmol}, 12$ mol\%) in THF at $15{ }^{\circ} \mathrm{C}$ under argon atmosphere and the mixture was stirred for $5 \mathrm{~min}$. Subsequently, $\alpha$-(trifluoromethyl)alkenyl acetates $2(0.75 \mathrm{mmol}, 1.5$ equiv.), pyrazolones $3(0.5$ mmol, 1.0 equiv.) and DBU (114 mg, $0.75 \mathrm{mmol}, 1.5$ equiv.) were added. The reaction mixture was stirred at at $\quad 15{ }^{\circ} \mathrm{C}$ for $36-60 \mathrm{~h}$. The solution was concentrated under reduced pressure and the crude was purified by column chromatography on silica gel to afford the pyrazolone-derived products 4 .

\section{Procedure for gram-scale synthesis of $4 \mathrm{a}$}

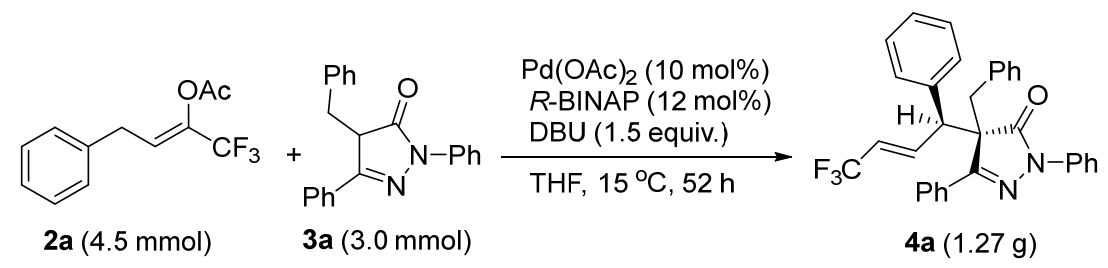

Dissolving the $\mathrm{Pd}(\mathrm{OAc})_{2}(67.4 \mathrm{mg}, 0.3 \mathrm{mmol}, 10 \mathrm{~mol} \%$ ) and $R$-BINAP (224.2 $\mathrm{mg}, 0.36 \mathrm{mmol}, 12$ mol\%) in $6 \mathrm{~mL}$ THF at $15{ }^{\circ} \mathrm{C}$ under argon atmosphere and the mixture was stirred for $5 \mathrm{~min}$. Subsequently, $\alpha$-(trifluoromethyl)alkenyl acetates 2 (1.10 g, 4.5 mmol, 1.5 equiv.), pyrazolones 3 (0.98 g, $3.0 \mathrm{mmol}, 1.0$ equiv.) and DBU (684.0 mg, $4.5 \mathrm{mmol}, 1.5$ equiv.) were added. The reaction mixture was stirred at at $15{ }^{\circ} \mathrm{C}$ for $52 \mathrm{~h}$. The solution was concentrated under reduced pressure and the crude was purified by column chromatography on silica gel to afford the products 4a $(1.27 \mathrm{~g}, 83 \%,>20: 1 \mathrm{dr}, 95 \%$ ee $)$.

\section{General procedures for the transformation of product 4 a}

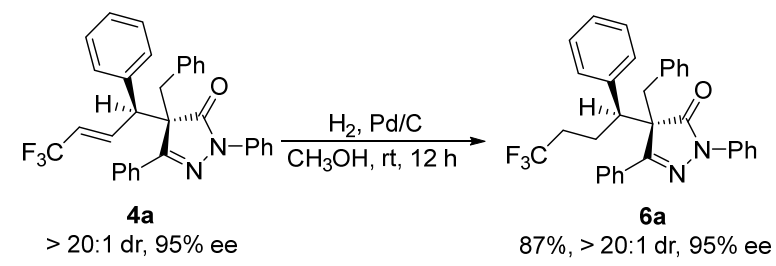

A solution of $4 \mathbf{a}(255.0 \mathrm{mg}, 0.5 \mathrm{mmol})$ and $10 \% \mathrm{Pd} / \mathrm{C}(25.5 \mathrm{mg}, 10 \% \mathrm{w} / \mathrm{w})$ in $3.0 \mathrm{~mL} \mathrm{CH}_{3} \mathrm{OH}$ was stirred at room temperature under a hydrogen atmosphere. The reaction mixture was stirred for about $12 \mathrm{~h}$ till completion (monitored by TLC). Then the mixture was filtered, concentrated under reduced pressure, and the crude was purified by column chromatography on silica gel to afford the desired product 6a $(87 \%$ yield, $>20: 1 \mathrm{dr}, 95 \%$ ee $)$. 


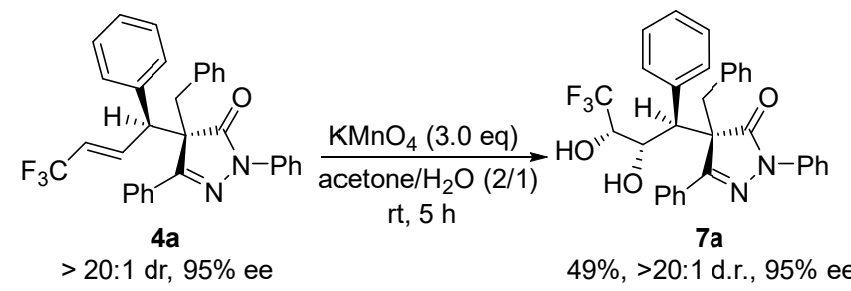

To a solution of $4 \mathbf{a}(204.0 \mathrm{mg}, 0.4 \mathrm{mmol})$ in acetone $/ \mathrm{H}_{2} \mathrm{O}(2: 1, \mathrm{v} / \mathrm{v}, 12 \mathrm{~mL})$ was added $\mathrm{KMnO}_{4}$ $(189.6 \mathrm{mg}, 1.2 \mathrm{mmol})$ at room temperature, and the mixture was stirred for $5 \mathrm{~h}$. The reaction mixture was concentrated under reduced pressure to remove acetone and extracted with $\mathrm{CH}_{2} \mathrm{Cl}_{2}$ for 3 times. The combined organic extracts were dried over $\mathrm{Na}_{2} \mathrm{SO}_{4}$, and the solvent was removed under reduced pressure. The crude product was purified by column chromatography on silica gel to afford the desired products $7 \mathbf{a}(49 \%$ yield, $>20: 1 \mathrm{dr}, 95 \%$ ee).

\section{Reference}

[1] H. Nakagawa, R. Ohyama, A. Kimata, T. Suzuki, N. Miyata, Bioorg. Med. Chem. Lett. 2006, 16, 5939-5942.

[2] A. Rioz-Martínez, A. Cuetos, C. Rodríguez, G. de Gonzalo, I. Lavandera, M. W. Fraaije, V. Gotor, Ange. Chem. Int.Ed. 2011, 50, 8387-8390.

[3] M. Kamlar, I. Císařová, J. Veselý, Org. Biomol. Chem. 2015, 13, 2884-2889.

[4] Y. Zhao, Y. Zhou, J. Liu, D. Yang, L. Tao, Y. Liu, X. Dong, J. Liu, J. Qu, J. Org. Chem. 2016, 81, 4797-4806.

\section{Crystal data and structure refinement for $4 a b$}

The crystals of $4 \mathbf{a b}$ were obtained by slow evaporation of the solution (in $\mathrm{CH}_{2} \mathrm{Cl}_{2} / n$-hexane (1:3) at $0{ }^{\circ} \mathrm{C}$.

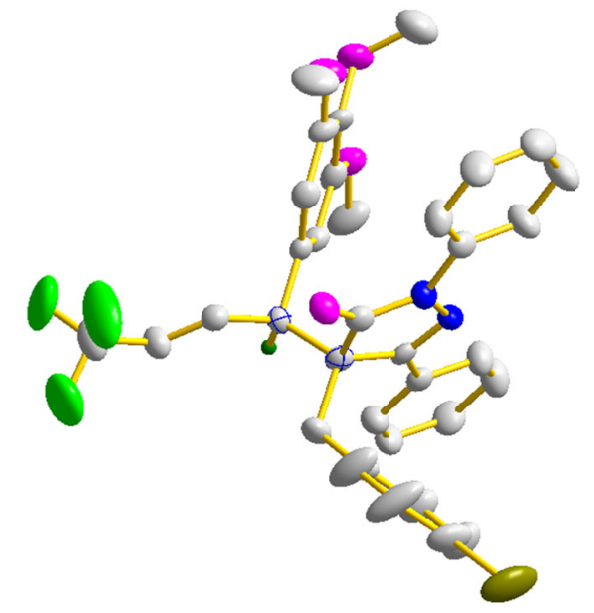

Figure S1. ORTEP diagrams of $\mathbf{4 a b}$ with thermal ellipsoids shown at a $50 \%$ probability level. Hydrogen atoms except for substituted on a chiral carbon atom are omitted for the sake of clarity. 
Identification code

Empirical formula

Formula weight

Temperature

Wavelength

Crystal system, space group

Unit cell dimensions

Volume

Z, Calculated density

Absorption coefficient

$\mathrm{F}(000)$

Crystal size

Theta range for data collection

Limiting indices

Reflections collected / unique

Completeness to theta $=24.999$

Absorption correction

Refinement method

Data / restraints / parameters

Goodness-of-fit on $\mathrm{F}^{\wedge} 2$

Final $\mathrm{R}$ indices [I $>2 \operatorname{sigma}(\mathrm{I})]$

$\mathrm{R}$ indices (all data)

Absolute structure parameter

Largest diff. peak and hole
$4 \mathrm{ab}$

$$
\mathrm{C}_{39} \mathrm{H}_{38} \mathrm{BrF}_{3} \mathrm{~N}_{2} \mathrm{O}_{5}
$$

751.62

173(2) K

$0.71073 \mathrm{~A}$

Orthorhombic, P2(1)2(1)2(1)

$$
\begin{array}{lc}
\mathrm{a}=11.3086(4) \mathrm{A} & \text { alpha }=90 \mathrm{deg} . \\
\mathrm{b}=11.5305(3) \mathrm{A} & \text { beta }=90 \mathrm{deg} . \\
\mathrm{c}=29.2389(9) \mathrm{A} & \text { gamma }=90 \mathrm{deg} .
\end{array}
$$

3812.6(2) $\mathrm{A}^{\wedge} 3$

4, $\quad 1.309 \mathrm{Mg} / \mathrm{m}^{\wedge} 3$

$1.136 \mathrm{~mm}^{\wedge}-1$

1552

$0.30 \times 0.04 \times 0.03 \mathrm{~mm}$

1.931 to $24.999 \mathrm{deg}$.

$-12<=\mathrm{h}<=13,-13<=\mathrm{k}<=13,-34<=\mathrm{l}<=34$

$33979 / 6716$ [R(int $)=0.0843]$

$99.9 \%$

Empirical

Full-matrix least-squares on $\mathrm{F}^{\wedge} 2$

$6716 / 0 / 406$

0.853

$\mathrm{R} 1=0.0460, \mathrm{wR} 2=0.1258$

$\mathrm{R} 1=0.0667, \mathrm{wR} 2=0.1361$

$0.087(7)$

0.339 and -0.420 e. $\mathrm{A}^{\wedge}-3$

\section{Experimental characterization data for new products}

1,1,1-Trifluoro-4-phenylbut-2-en-2-yl acetate (2a)

OAc Column chromatography elution: petroleum ether/ethyl acetate $=100: 1(\mathrm{v} / \mathrm{v})$, $\mathrm{CF}_{3}$ colorless oil, $2.10 \mathrm{~g}, 86 \%$ yield. 
${ }^{1} \mathrm{H}$ NMR $\left(400 \mathrm{MHz}, \mathrm{CDCl}_{3}\right) \delta 7.38-7.33(\mathrm{~m}, 2 \mathrm{H}), 7.30-7.28(\mathrm{~m}, 1 \mathrm{H}), 7.22(\mathrm{~d}, J=7.2 \mathrm{~Hz}, 2 \mathrm{H})$, $6.26(\mathrm{t}, J=7.4 \mathrm{~Hz}, 1 \mathrm{H}), 3.39$ (dd, $J=7.3,1.3 \mathrm{~Hz}, 2 \mathrm{H}), 2.30$ (s, 3H). ${ }^{13} \mathrm{C}$ NMR (101 MHz, $\left.\mathrm{CDCl}_{3}\right)$ $\delta 167.3,137.2,135.24(\mathrm{q}, J=36.4 \mathrm{~Hz}), 128.8,128.6,126.9,124.8(\mathrm{q}, J=3.0 \mathrm{~Hz}), 119.8(\mathrm{q}, J=$ $273.7 \mathrm{~Hz}$ ), 31.7, 20.1. ${ }^{19} \mathrm{~F}$ NMR $\left(470 \mathrm{MHz}, \mathrm{CDCl}_{3}\right) \delta-70.85$ (s). HRMS (EI) $\mathrm{m} / z$ : calcd for $\mathrm{C}_{12} \mathrm{H}_{11} \mathrm{~F}_{3} \mathrm{O}_{2}\left[\mathrm{M}^{+\bullet}\right]$ 244.0711, found: 244.0710.

\section{1,1,1-Trifluoro-4-(o-tolyl)but-2-en-2-yl acetate (2b)}

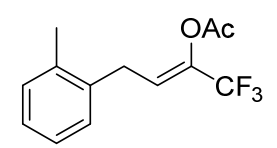

Column chromatography elution: petroleum ether/ethyl acetate $=100: 1(\mathrm{v} / \mathrm{v})$, colorless oil, $2.14 \mathrm{~g}, 83 \%$ yield.

${ }^{1} \mathrm{H}$ NMR (400 MHz, $\left.\mathrm{CDCl}_{3}\right) \delta 7.22-7.18(\mathrm{~m}, 3 \mathrm{H}), 7.17$ - $7.12(\mathrm{~m}, 1 \mathrm{H}), 6.20$ (t, $J=7.2 \mathrm{~Hz}, 1 \mathrm{H}), 3.37$ (dd, $J=7.1,1.7 \mathrm{~Hz}, 2 \mathrm{H}), 2.31$ (s, 3H), 2.29 (s, 3H). ${ }^{13} \mathrm{C}$ NMR $(101 \mathrm{MHz}$, $\left.\mathrm{CDCl}_{3}\right) \delta 167.3,136.3,135.6,135.2(\mathrm{q}, J=35.8 \mathrm{~Hz}), 130.5,129.1,127.2,126.4,124.4$ (q, $J=3.3$ $\mathrm{Hz}), 119.8$ (q, $J=272.8 \mathrm{~Hz}), 29.5,20.1,19.4 .{ }^{19} \mathrm{~F}$ NMR $\left(470 \mathrm{MHz}, \mathrm{CDCl}_{3}\right) \delta-70.78$ (s). HRMS (EI) $m / z$ : calcd for $\mathrm{C}_{13} \mathrm{H}_{13} \mathrm{~F}_{3} \mathrm{O}_{2}\left[\mathrm{M}^{+\bullet}\right] 258.0868$, found: 258.0874 .

\section{1,1,1-Trifluoro-4-( $m$-tolyl)but-2-en-2-yl acetate (2c)}

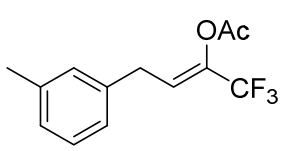

Column chromatography elution: petroleum ether/ethyl acetate $=100: 1(\mathrm{v} / \mathrm{v})$, colorless oil, $2.17 \mathrm{~g}, 84 \%$ yield.

${ }^{1} \mathrm{H}$ NMR $\left(400 \mathrm{MHz}, \mathrm{CDCl}_{3}\right) \delta 7.21-7.15(\mathrm{~m}, 1 \mathrm{H}), 7.04(\mathrm{~d}, J=7.6 \mathrm{~Hz}, 1 \mathrm{H})$, $6.97-6.95(\mathrm{~m}, 2 \mathrm{H}), 6.20(\mathrm{t}, J=7.4 \mathrm{~Hz}, 1 \mathrm{H}), 3.30(\mathrm{dd}, J=7.4,1.7 \mathrm{~Hz}, 2 \mathrm{H}), 2.31(\mathrm{~s}, 3 \mathrm{H}), 2.24(\mathrm{~s}$, $3 \mathrm{H}) .{ }^{13} \mathrm{C}$ NMR $\left(101 \mathrm{MHz}, \mathrm{CDCl}_{3}\right) \delta 167.3,138.5,137.1,135.1$ (q, $\left.J=36.4 \mathrm{~Hz}\right), 129.3,128.7$, 127.7, 125.6, 124.9 (q, $J=3.0 \mathrm{~Hz}), 119.8$ (q, $J=272.5 \mathrm{~Hz}), 31.6,21.3,20.2 .{ }^{19} \mathrm{~F}$ NMR (377 MHz, $\left.\mathrm{CDCl}_{3}\right) \delta-70.83$ (s). HRMS (EI) $\mathrm{m} / z$ : calcd for $\mathrm{C}_{13} \mathrm{H}_{13} \mathrm{~F}_{3} \mathrm{O}_{2}\left[\mathrm{M}^{+\bullet}\right]$ 258.0868, found: 258.0863 .

\section{1,1,1-Trifluoro-4-( $p$-tolyl)but-2-en-2-yl acetate (2d)}

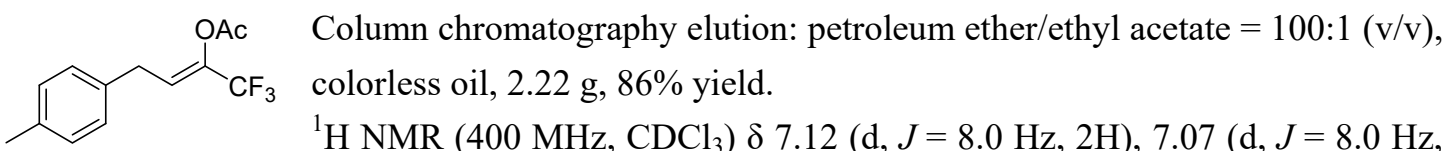
2H), $6.21(\mathrm{t}, J=7.4 \mathrm{~Hz}, 1 \mathrm{H}), 3.32(\mathrm{dd}, J=7.4,1.5 \mathrm{~Hz}, 2 \mathrm{H}), 2.32(\mathrm{~s}, 3 \mathrm{H}), 2.26(\mathrm{~s}, 3 \mathrm{H}) .{ }^{13} \mathrm{C} \mathrm{NMR}$ $\left(101 \mathrm{MHz}, \mathrm{CDCl}_{3}\right) \delta 167.3,136.5,135.0(\mathrm{q}, J=36.3 \mathrm{~Hz}), 134.1,129.5,128.4,125.1$ (q, $J=3.5$ Hz), 119.9 (q, $J=272.5 \mathrm{~Hz}$ ), 31.3, 21.0, 20.1. ${ }^{19} \mathrm{~F}$ NMR (377 MHz, $\mathrm{CDCl}_{3}$ ) $\delta-70.84$ (s). HRMS (EI) $m / z$ : calcd for $\mathrm{C}_{13} \mathrm{H}_{13} \mathrm{~F}_{3} \mathrm{O}_{2}\left[\mathrm{M}^{+\bullet}\right] 258.0868$, found: 258.0871 .

\section{1,1,1-Trifluoro-4-(4-isopropylphenyl)but-2-en-2-yl acetate (2e)}

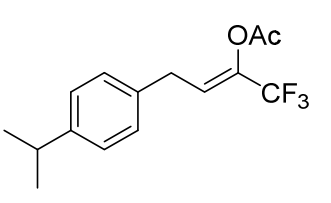

Column chromatography elution: petroleum ether/ethyl acetate $=100: 1$ (v/v), colorless oil, $2.57 \mathrm{~g}, 90 \%$ yield. ${ }^{1} \mathrm{H}$ NMR $\left(400 \mathrm{MHz}, \mathrm{CDCl}_{3}\right) \delta 7.19$ 
(d, $J=8.1 \mathrm{~Hz}, 2 \mathrm{H}), 7.12(\mathrm{~d}, J=8.1 \mathrm{~Hz}, 2 \mathrm{H}), 6.24$ (t, $J=7.2 \mathrm{~Hz}, 1 \mathrm{H}), 3.34$ (dd, $J=7.3,1.6 \mathrm{~Hz}$, 2H), 2.89 (heptet, $J=6.9 \mathrm{~Hz}, 1 \mathrm{H}), 2.27(\mathrm{~s}, 3 \mathrm{H}), 1.25(\mathrm{~d}, J=6.9 \mathrm{~Hz}, 6 \mathrm{H}) .{ }^{13} \mathrm{C}$ NMR $(101 \mathrm{MHz}$, $\left.\mathrm{CDCl}_{3}\right) \delta 167.3,147.6,135.1(\mathrm{q}, J=36.3 \mathrm{~Hz}), 134.5,128.5,126.9,125.0(\mathrm{q}, J=3.5 \mathrm{~Hz}), 119.9(\mathrm{q}$, $J=272.4 \mathrm{~Hz}$ ), 33.8, 31.3, 24.0, 20.1. ${ }^{19} \mathrm{~F}$ NMR $\left(377 \mathrm{MHz}, \mathrm{CDCl}_{3}\right) \delta-70.82$ (s). HRMS (EI) $m / z$ : calcd for $\mathrm{C}_{15} \mathrm{H}_{17} \mathrm{~F}_{3} \mathrm{O}_{2}\left[\mathrm{M}^{+\bullet}\right] 286.1181$, found: 286.1187 .

\section{1,1,1-Trifluoro-4-(2-methoxyphenyl)but-2-en-2-yl acetate (2f)}

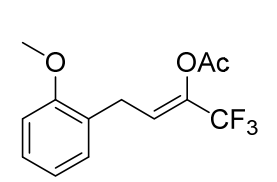

Column chromatography elution: petroleum ether/ethyl acetate $=100: 1(\mathrm{v} / \mathrm{v})$, light yellow oil, $2.19 \mathrm{~g}, 80 \%$ yield.

${ }^{1} \mathrm{H}$ NMR (400 MHz, $\left.\mathrm{CDCl}_{3}\right) \delta 7.22(\mathrm{~d}, J=7.8 \mathrm{~Hz}, 1 \mathrm{H}), 7.09(\mathrm{~d}, J=7.2 \mathrm{~Hz}$, $1 \mathrm{H}), 6.91-6.84(\mathrm{~m}, 2 \mathrm{H}), 6.24(\mathrm{t}, J=7.1 \mathrm{~Hz}, 1 \mathrm{H}), 3.82(\mathrm{~s}, 3 \mathrm{H}), 3.33$ (d, $J=7.1$ $\mathrm{Hz}, 2 \mathrm{H}), 2.25$ (s, 3H). ${ }^{13} \mathrm{C} \mathrm{NMR}\left(101 \mathrm{MHz}, \mathrm{CDCl}_{3}\right) \delta 167.4,157.3,134.9$ (q, $\left.J=36.4 \mathrm{~Hz}\right), 129.9$, $128.3,125.5,124.7$ (q, $J=3.6 \mathrm{~Hz}), 120.7,120.0(\mathrm{q}, J=273.7 \mathrm{~Hz}), 110.4,55.3,26.5,20.1 .{ }^{19} \mathrm{~F}$ NMR (377 MHz, $\left.\mathrm{CDCl}_{3}\right) \delta-70.69$ (s). HRMS (EI) $m / z$ : calcd for $\mathrm{C}_{13} \mathrm{H}_{13} \mathrm{~F}_{3} \mathrm{O}_{3}\left[\mathrm{M}^{+\bullet}\right.$ ] 274.0817, found: 274.0824 .

\section{1,1,1-Trifluoro-4-(naphthalen-2-yl)but-2-en-2-yl acetate (2g)}

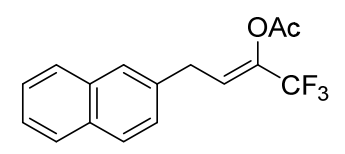

Column chromatography elution: petroleum ether/ethyl acetate $=50: 1$ (v/v), light yellow oil, $2.50 \mathrm{~g}, 85 \%$ yield. ${ }^{1} \mathrm{H}$ NMR $\left(400 \mathrm{MHz}, \mathrm{CDCl}_{3}\right) \delta$ $7.83-7.81(\mathrm{~m}, 3 \mathrm{H}), 7.64(\mathrm{~s}, 1 \mathrm{H}), 7.51-7.47(\mathrm{~m}, 2 \mathrm{H}), 7.32(\mathrm{~d}, J=8.3 \mathrm{~Hz}$, $1 \mathrm{H}), 6.33(\mathrm{t}, J=7.1 \mathrm{~Hz}, 1 \mathrm{H}), 3.54(\mathrm{~d}, J=7.1 \mathrm{~Hz}, 2 \mathrm{H}), 2.29$ (s, 3H). ${ }^{13} \mathrm{C} \mathrm{NMR}\left(101 \mathrm{MHz}, \mathrm{CDCl}_{3}\right)$ $\delta 167.4,135.4(\mathrm{q}, J=36.3 \mathrm{~Hz}), 134.7,133.6,132.4,128.6,127.7,127.6,127.0,126.8,126.3$, 125.8, 124.7 (d, $J=3.6 \mathrm{~Hz}), 119.9(\mathrm{~d}, J=272.7 \mathrm{~Hz}), 31.9,20.2 .{ }^{19} \mathrm{~F}$ NMR $\left(377 \mathrm{MHz}, \mathrm{CDCl}_{3}\right) \delta$ -70.78 (s). HRMS (EI) $m / z$ : calcd for $\mathrm{C}_{16} \mathrm{H}_{13} \mathrm{~F}_{3} \mathrm{O}_{2}\left[\mathrm{M}^{+}{ }^{*}\right]$ 294.0868, found: 294.0875.

\section{1,1,1-Trifluoro-4-([1,1'-biphenyl]-4-yl)but-2-en-2-yl acetate (2h)}

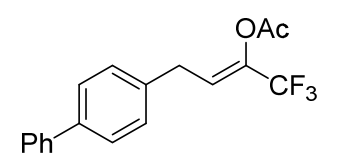

Column chromatography elution: petroleum ether/ethyl acetate $=50: 1(\mathrm{v} / \mathrm{v})$, white solid, mp $45-46{ }^{\circ} \mathrm{C}, 2.75 \mathrm{~g}, 86 \%$ yield. ${ }^{1} \mathrm{H}$ NMR (400 MHz, $\mathrm{CDCl}_{3}$ ) $\delta 7.65-7.56(\mathrm{~m}, 4 \mathrm{H}), 7.50-7.46(\mathrm{~m}, 2 \mathrm{H}), 7.40-7.37(\mathrm{~m}, 1 \mathrm{H}), 7.32-$ $7.30(\mathrm{~m}, 2 \mathrm{H}), 6.31(\mathrm{t}, J=7.2 \mathrm{~Hz}, 1 \mathrm{H}), 3.45(\mathrm{dd}, J=7.3,1.6 \mathrm{~Hz}, 2 \mathrm{H}), 2.31(\mathrm{~s}, 3 \mathrm{H}) .{ }^{13} \mathrm{C}$ NMR $(101$ $\left.\mathrm{MHz}, \mathrm{CDCl}_{3}\right) \delta 167.4,140.8,140.0,136.3,135.4(\mathrm{q}, J=36.3 \mathrm{~Hz}), 129.0,128.9,127.6,127.4$, 127.1, 124.7 (q, $J=3.6 \mathrm{~Hz}), 119.9(\mathrm{q}, J=271.3 \mathrm{~Hz}), 31.4,20.1 .{ }^{19} \mathrm{~F}$ NMR $\left(377 \mathrm{MHz}, \mathrm{CDCl}_{3}\right) \delta$ -70.75 (s). HRMS (EI) $m / z$ : calcd for $\mathrm{C}_{18} \mathrm{H}_{15} \mathrm{~F}_{3} \mathrm{O}_{2}\left[\mathrm{M}^{+\bullet}\right]$ 320.1024, found: 320.1032 . 


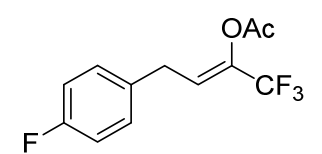

Column chromatography elution: petroleum ether/ethyl acetate $=100: 1$ (v/v), colorless oil, $2.33 \mathrm{~g}, 89 \%$ yield. ${ }^{1} \mathrm{H}$ NMR $\left(400 \mathrm{MHz}, \mathrm{CDCl}_{3}\right) \delta 7.17-$ $7.13(\mathrm{~m}, 2 \mathrm{H}), 7.04-6.97(\mathrm{~m}, 2 \mathrm{H}), 6.19(\mathrm{t}, J=7.4 \mathrm{~Hz}, 1 \mathrm{H}), 3.34$ (d, $J=7.4$ $\mathrm{Hz}, 2 \mathrm{H}), 2.27$ (s, 3H). ${ }^{13} \mathrm{C}$ NMR $\left(101 \mathrm{MHz}, \mathrm{CDCl}_{3}\right) \delta 167.3,161.9$ (d, $\left.J=245.2 \mathrm{~Hz}\right), 135.3$ (q, $J=$ $36.4 \mathrm{~Hz}), 132.9,130.0$ (d, $J=8.0 \mathrm{~Hz}), 124.5$ (q, $J=3.5 \mathrm{~Hz}), 119.8$ (q, $J=272.5 \mathrm{~Hz}), 115.6(\mathrm{~d}, J=$ $21.4 \mathrm{~Hz}$ ), 30.9, 20.0. ${ }^{19} \mathrm{~F}$ NMR (377 MHz, $\mathrm{CDCl}_{3}$ ) $\delta-70.93$ (s, 3F), -115.88 - -116.04 (m, 1F). HRMS (EI) $m / z$ : calcd for $\mathrm{C}_{12} \mathrm{H}_{10} \mathrm{~F}_{4} \mathrm{O}_{2}\left[\mathrm{M}^{+}\right]$262.0617, found: 262.0620 .

1,1,1-Trifluoro-4-(3-chlorophenyl)but-2-en-2-yl acetate (2j)

$\begin{array}{ll}\mathrm{OAc} & \text { Column chromatography elution: petroleum ether/ethyl acetate }=100: 1 \\ \mathrm{CF}_{3} & (\mathrm{v} / \mathrm{v}) \text {, colorless oil, } 2.42 \mathrm{~g}, 87 \% \text { yield. }{ }^{1} \mathrm{H} \mathrm{NMR}\left(400 \mathrm{MHz}, \mathrm{CDCl}_{3}\right) \delta 7.26-\end{array}$ $7.21(\mathrm{~m}, 2 \mathrm{H}), 7.16(\mathrm{~s}, 1 \mathrm{H}), 7.09-7.03(\mathrm{~m}, 1 \mathrm{H}), 6.18(\mathrm{t}, J=7.3 \mathrm{~Hz}, 1 \mathrm{H})$, $3.32(\mathrm{dd}, J=7.4,1.7 \mathrm{~Hz}, 2 \mathrm{H}), 2.26(\mathrm{~s}, 3 \mathrm{H}) .{ }^{13} \mathrm{C}$ NMR $\left(101 \mathrm{MHz}, \mathrm{CDCl}_{3}\right) \delta 167.2,139.2,135.7$ (q, $J=36.5 \mathrm{~Hz}), 134.5,130.0,128.7,127.2,126.7,123.9(\mathrm{q}, J=3.6 \mathrm{~Hz}), 119.7(\mathrm{q}, J=272.6 \mathrm{~Hz})$, 31.3, 20.1. ${ }^{19} \mathrm{~F}$ NMR $\left(377 \mathrm{MHz}, \mathrm{CDCl}_{3}\right) \delta-70.94$ (s). HRMS (EI) $\mathrm{m} / \mathrm{z}$ : calcd for $\mathrm{C}_{12} \mathrm{H}_{10} \mathrm{ClF}_{3} \mathrm{O}_{2}$ $\left[\mathrm{M}^{+}{ }^{\bullet}\right.$ 2 278.0321, found: 278.0324 .

1,1,1-Trifluoro-4-(4-bromophenyl)but-2-en-2-yl acetate (2k)

$\begin{array}{ll}\mathrm{CF}_{3} & \text { Column chromatography elution: petroleum ether/ethyl acetate }=100: 1 \\ & (\mathrm{v} / \mathrm{v}), \text { light yellow oil, } 2.75 \mathrm{~g}, 85 \% \text { yield. }{ }^{1} \mathrm{H} \text { NMR }\left(400 \mathrm{MHz}, \mathrm{CDCl}_{3}\right) \delta\end{array}$ $3.32(\mathrm{~d}, J=7.3 \mathrm{~Hz}, 2 \mathrm{H}), 2.27$ (s, 3H). ${ }^{13} \mathrm{C}$ NMR $\left(101 \mathrm{MHz}, \mathrm{CDCl}_{3}\right) \delta 167.2,136.2,135.6$ (q, $J=$ $36.5 \mathrm{~Hz}), 131.9,130.3,124.0$ (q, $J=3.5 \mathrm{~Hz}), 120.8,119.7$ (q, $J=273.7 \mathrm{~Hz}), 31.1,20.1 .{ }^{19} \mathrm{~F}$ NMR (377 MHz, $\mathrm{CDCl}_{3}$ ) $\delta-70.93$ (s). HRMS (EI) $\mathrm{m} / z$ : calcd for $\mathrm{C}_{12} \mathrm{H}_{10} \mathrm{BrF}_{3} \mathrm{O}_{2}\left[\mathrm{M}^{+}{ }^{\bullet}\right] 321.9816$, found: 321.9819 .

1,1,1-Trifluoro-4-(3-(trifluoromethyl)phenyl)but-2-en-2-yl acetate (2I)

Column chromatography elution: petroleum ether/ethyl acetate $=100: 1$
$(\mathrm{v} / \mathrm{v})$, colorless oil, $2.81 \mathrm{~g}, 90 \%$ yield. ${ }^{1} \mathrm{H} \mathrm{NMR}\left(400 \mathrm{MHz}, \mathrm{CDCl}_{3}\right) \delta 7.52$
$(\mathrm{~d}, J=7.6 \mathrm{~Hz}, 1 \mathrm{H}), 7.47-7.42(\mathrm{~m}, 2 \mathrm{H}), 7.39(\mathrm{~d}, J=7.6 \mathrm{~Hz}, 1 \mathrm{H}), 6.21(\mathrm{t}, J$ $=7.3 \mathrm{~Hz}, 1 \mathrm{H}), 3.42(\mathrm{~d}, J=7.2 \mathrm{~Hz}, 2 \mathrm{H}), 2.27(\mathrm{~s}, 3 \mathrm{H}) .{ }^{13} \mathrm{C} \mathrm{NMR}\left(101 \mathrm{MHz}, \mathrm{CDCl}_{3}\right) \delta 167.2,138.2$, 135.9 (q, $J=36.6 \mathrm{~Hz}), 131.9,131.1(\mathrm{q}, J=32.2 \mathrm{~Hz}), 129.3,125.3(\mathrm{q}, J=3.8 \mathrm{~Hz}), 124.0(\mathrm{q}, J=$ $272.7 \mathrm{~Hz}), 123.8(\mathrm{q}, J=3.8 \mathrm{~Hz}), 123.6(\mathrm{q}, J=3.5 \mathrm{~Hz}), 119.7(\mathrm{q}, J=272.7 \mathrm{~Hz}), 31.4,20.0 .{ }^{19} \mathrm{~F}$ NMR (377 MHz, $\left.\mathrm{CDCl}_{3}\right) \delta-62.72(\mathrm{~s}, 3 \mathrm{~F}),-71.01(\mathrm{~s}, 3 \mathrm{~F})$. HRMS (EI) $\mathrm{m} / \mathrm{z}$ : calcd for $\mathrm{C}_{13} \mathrm{H}_{10} \mathrm{~F}_{6} \mathrm{O}_{2}$ $\left[\mathrm{M}^{+\bullet}\right]$ 312.0585, found: 312.0587 . 
OAC Column chromatography elution: petroleum ether/ethyl acetate $=20: 1$ - $\mathrm{CF}_{3} \quad(\mathrm{v} / \mathrm{v})$, white solid, $\mathrm{mp} 67-68^{\circ} \mathrm{C}, 1.67 \mathrm{~g}, 62 \%$ yield. ${ }^{1} \mathrm{H}$ NMR $(400 \mathrm{MHz}$, $\left.\mathrm{NC} \mathrm{CDCl}_{3}\right) \delta 7.55(\mathrm{~d}, J=8.0 \mathrm{~Hz}, 2 \mathrm{H}), 7.30(\mathrm{~d}, J=8.0 \mathrm{~Hz}, 2 \mathrm{H}), 6.18(\mathrm{t}, J=7.4 \mathrm{~Hz}$, $1 \mathrm{H}), 3.42(\mathrm{~d}, J=7.4 \mathrm{~Hz}, 2 \mathrm{H}), 2.24(\mathrm{~s}, 3 \mathrm{H}) .{ }^{13} \mathrm{C}$ NMR $\left(101 \mathrm{MHz}, \mathrm{CDCl}_{3}\right) \delta 167.2,142.7,136.1$ (q, $J=$ $36.3 \mathrm{~Hz}), 132.4,129.4,123.1(\mathrm{q}, J=3.5 \mathrm{~Hz}), 119.6(\mathrm{q}, J=273.7 \mathrm{~Hz}), 118.6,110.8,31.5,20.0 .{ }^{19} \mathrm{~F}$ NMR (377 MHz, $\left.\mathrm{CDCl}_{3}\right) \delta-71.00$ (s). HRMS (EI) m/z: calcd for $\mathrm{C}_{13} \mathrm{H}_{10} \mathrm{~F}_{3} \mathrm{NO}_{2}\left[\mathrm{M}^{+}\right]$269.0664, found: 269.0658 .

1,1,1-Trifluoro-4-(3,4,5-trimethoxyphenyl)but-2-en-2-yl acetate (2n)

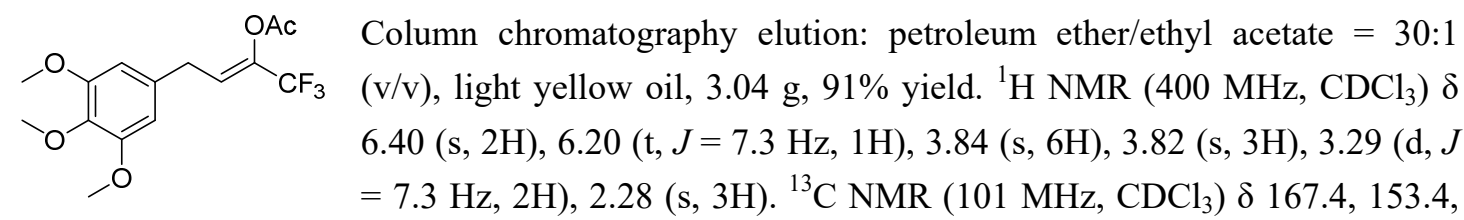
136.9, $135.2(\mathrm{q}, J=36.4 \mathrm{~Hz}), 132.8,124.7$ (q, $J=3.5 \mathrm{~Hz}), 119.8$ (q, $J=272.7 \mathrm{~Hz}), 105.5,60.8$, 56.1, 31.9, 20.2. ${ }^{19} \mathrm{~F}$ NMR (377 MHz, $\left.\mathrm{CDCl}_{3}\right) \delta-70.82$ (s). HRMS (EI) $m / z$ : calcd for $\mathrm{C}_{15} \mathrm{H}_{17} \mathrm{~F}_{3} \mathrm{O}_{5}$ $\left[\mathrm{M}^{+\bullet}\right]$ 334.1028, found: 334.1038 .

1,1,1-Trifluoro-4-(thiophen-2-yl)but-2-en-2-yl acetate (2o)

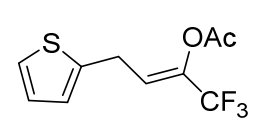

Column chromatography elution: petroleum ether/ethyl acetate $=100: 1(\mathrm{v} / \mathrm{v})$, light yellow oil, $2.20 \mathrm{~g}, 88 \%$ yield.

${ }^{1} \mathrm{H}$ NMR $\left(400 \mathrm{MHz}, \mathrm{CDCl}_{3}\right) \delta 7.18(\mathrm{dd}, J=5.2,1.1 \mathrm{~Hz}, 1 \mathrm{H}), 6.96-6.94(\mathrm{~m}$, $1 \mathrm{H}), 6.87-6.84(\mathrm{~m}, 1 \mathrm{H}), 6.26(\mathrm{t}, J=7.3 \mathrm{~Hz}, 1 \mathrm{H}), 3.57(\mathrm{dd}, J=7.3,1.6 \mathrm{~Hz}, 2 \mathrm{H}), 2.28(\mathrm{~s}, 3 \mathrm{H}) .{ }^{13} \mathrm{C}$ $\operatorname{NMR}\left(101 \mathrm{MHz}, \mathrm{CDCl}_{3}\right) \delta 167.1,139.1,135.3(\mathrm{q}, J=36.6 \mathrm{~Hz}), 127.2,125.7,124.4,123.9(\mathrm{q}, J=$ $3.5 \mathrm{~Hz}$ ), 119.7 (q, $\left.J=272.6 \mathrm{~Hz}), 25.9,20.1 .{ }^{19} \mathrm{~F} \mathrm{NMR} \mathrm{(377} \mathrm{MHz,} \mathrm{CDCl}_{3}\right) \delta-71.00$ (s). HRMS (EI) $\mathrm{m} / \mathrm{z}$ : calcd for $\mathrm{C}_{10} \mathrm{H}_{9} \mathrm{~F}_{3} \mathrm{O}_{2} \mathrm{~S}\left[\mathrm{M}^{+}\right.$] 250.0275 , found: 250.0277 .

1,1,1-Trifluorodec-2-en-2-yl acetate (2p)

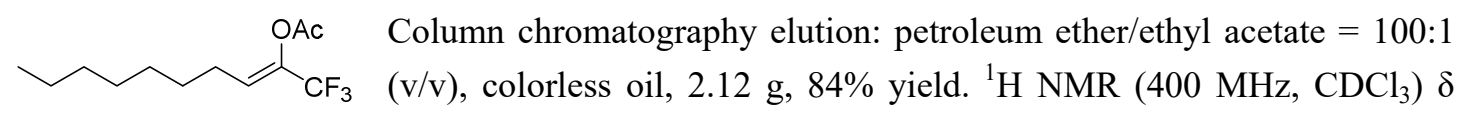
$6.04(\mathrm{t}, J=7.5 \mathrm{~Hz}, 1 \mathrm{H}), 2.23(\mathrm{~s}, 3 \mathrm{H}), 2.04-1.95(\mathrm{~m}, 2 \mathrm{H}), 1.43-1.38(\mathrm{~m}, 2 \mathrm{H}), 1.34-1.21(\mathrm{~m}$, $8 \mathrm{H}), 0.91-0.81(\mathrm{~m}, 3 \mathrm{H}) .{ }^{13} \mathrm{C} \mathrm{NMR}\left(101 \mathrm{MHz}, \mathrm{CDCl}_{3}\right) \delta 167.2,134.7$ (q, $\left.J=36.1 \mathrm{~Hz}\right), 126.2(\mathrm{q}$, $J=3.5 \mathrm{~Hz}), 119.9$ (q, $J=272.1 \mathrm{~Hz}), 31.7,29.1,28.9,27.8,25.4,22.6,20.1,14.0 .{ }^{19} \mathrm{~F}$ NMR $(377$ $\left.\mathrm{MHz}, \mathrm{CDCl}_{3}\right) \delta-70.95$ (s). HRMS (EI) $\mathrm{m} / \mathrm{z}$ : calcd for $\mathrm{C}_{12} \mathrm{H}_{19} \mathrm{~F}_{3} \mathrm{O}_{2}\left[\mathrm{M}^{+\bullet}\right] 252.1337$, found: 252.1339 . 


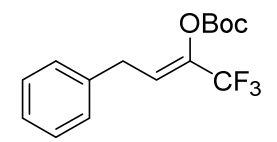

Column chromatography elution: petroleum ether/ethyl acetate $=100: 1(\mathrm{v} / \mathrm{v})$, colorless oil, $2.51 \mathrm{~g}, 83 \%$ yield.

${ }^{1} \mathrm{H}$ NMR $\left(400 \mathrm{MHz}, \mathrm{CDCl}_{3}\right) \delta 7.35-7.30(\mathrm{~m}, 2 \mathrm{H}), 7.29-7.23(\mathrm{~m}, 1 \mathrm{H}), 7.21$ $(\mathrm{d}, J=7.2 \mathrm{~Hz}, 2 \mathrm{H}), 6.20(\mathrm{t}, J=7.5 \mathrm{~Hz}, 1 \mathrm{H}), 3.44(\mathrm{dd}, J=7.5,1.5 \mathrm{~Hz}, 2 \mathrm{H}), 1.55(\mathrm{~s}, 9 \mathrm{H}) .{ }^{13} \mathrm{C}$ NMR $\left(101 \mathrm{MHz}, \mathrm{CDCl}_{3}\right) \delta 149.9,137.2,135.7(\mathrm{q}, J=36.5 \mathrm{~Hz}), 128.8,128.6,126.9,124.8(\mathrm{q}, J=3.3$ $\mathrm{Hz}), 119.8$ (q, $J=272.6 \mathrm{~Hz}), 84.9,31.4,27.5 .{ }^{19} \mathrm{~F}$ NMR $\left(377 \mathrm{MHz}, \mathrm{CDCl}_{3}\right) \delta-70.68$ (s). HRMS (EI) $\mathrm{m} / \mathrm{z}$ : calcd for $\mathrm{C}_{11} \mathrm{H}_{8} \mathrm{~F}_{3} \mathrm{O}_{2}[\mathrm{M}-\mathrm{OBu}]^{+} 229.0476$, found: 229.0478 .

\section{1,1,1-Trifluoro-4-phenylbut-2-en-2-yl benzoate (2-OBz)}

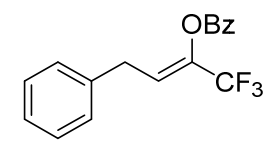

Column chromatography elution: petroleum ether/ethyl acetate $=50: 1(\mathrm{v} / \mathrm{v})$, colorless oil, $2.48 \mathrm{~g}, 81 \%$ yield.

${ }^{1} \mathrm{H}$ NMR $\left(400 \mathrm{MHz}, \mathrm{CDCl}_{3}\right) \delta 8.03-7.97(\mathrm{~m}, 2 \mathrm{H}), 7.75-7.67(\mathrm{~m}, 1 \mathrm{H}), 7.58$ $(\mathrm{dd}, J=10.8,4.9 \mathrm{~Hz}, 2 \mathrm{H}), 7.33$ (dd, $J=9.9,4.5 \mathrm{~Hz}, 2 \mathrm{H}), 7.29-7.19$ (m, 3H), $6.34(\mathrm{t}, J=7.5 \mathrm{~Hz}$, $1 \mathrm{H}), 3.70(\mathrm{dd}, J=7.5,1.8 \mathrm{~Hz}, 2 \mathrm{H}) .{ }^{13} \mathrm{C} \mathrm{NMR}\left(101 \mathrm{MHz}, \mathrm{CDCl}_{3}\right) \delta 163.3,137.3,135.4(\mathrm{~d}, J=$ $36.6 \mathrm{~Hz}), 134.3,130.5,130.3,128.8,128.6,127.9,126.9,125.1(\mathrm{~d}, J=3.5 \mathrm{~Hz}), 119.9(\mathrm{~d}, J=$ $272.8 \mathrm{~Hz}), 31.8 .{ }^{19} \mathrm{~F}$ NMR $\left(377 \mathrm{MHz}, \mathrm{CDCl}_{3}\right) \delta-70.59(\mathrm{~s})$.

HRMS (EI) $m / z$ : calcd for $\mathrm{C}_{17} \mathrm{H}_{13} \mathrm{~F}_{3} \mathrm{O}_{2}\left[\mathrm{M}^{+\bullet}\right]$ 306.0868, found: 306.0865 .

\section{1,1,1-Trifluoro-4-phenylbut-2-en-2-yl 4-methylbenzenesulfonate (2-OTs)}

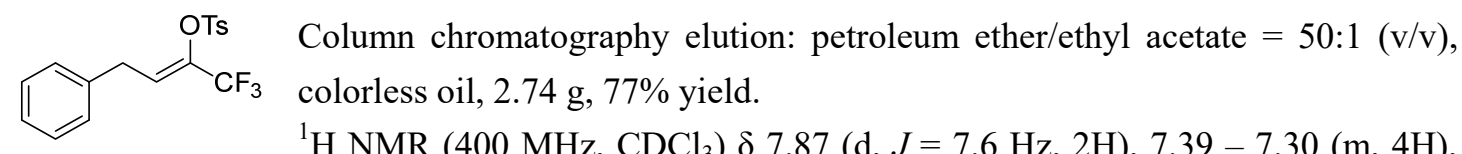
$7.26-7.20$ (m, 3H), 6.33 (t, $J=7.4 \mathrm{~Hz}, 1 \mathrm{H}), 3.69$ (d, $J=7.3 \mathrm{~Hz}, 2 \mathrm{H}), 2.46(\mathrm{~s}, 3 \mathrm{H}) .{ }^{13} \mathrm{C}$ NMR $(101$ $\left.\mathrm{MHz}, \mathrm{CDCl}_{3}\right) \delta 145.9,137.1,134.7(\mathrm{~d}, J=37.3 \mathrm{~Hz}), 133.2,129.9,128.8,128.65(\mathrm{q}, J=3.3 \mathrm{~Hz})$, 128.6, 128.2, 127.0, 119.4 (d, $J=273.2 \mathrm{~Hz}), 32.3,21.7 .{ }^{19} \mathrm{~F} \mathrm{NMR}\left(377 \mathrm{MHz}, \mathrm{CDCl}_{3}\right) \delta-69.02(\mathrm{~s})$. HRMS (EI) $m / z$ : calcd for $\mathrm{C}_{17} \mathrm{H}_{15} \mathrm{~F}_{3} \mathrm{O}_{3} \mathrm{~S}\left[\mathrm{M}^{+\bullet}\right]$ 356.0694, found: 356.0704 .

(E)-1,1,1-Trifluoro-4-phenylbut-3-en-2-yl acetate (2a')

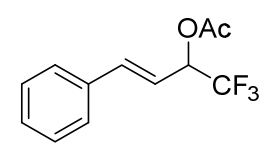

${ }^{1} \mathrm{H}$ NMR (400 MHz, $\left.\mathrm{CDCl}_{3}\right) \delta 7.43(\mathrm{dd}, J=7.9,1.3 \mathrm{~Hz}, 2 \mathrm{H}), 7.40-7.30(\mathrm{~m}$, $3 \mathrm{H}), 6.87(\mathrm{~d}, J=15.9 \mathrm{~Hz}, 1 \mathrm{H}), 6.13(\mathrm{dd}, J=15.9,7.8 \mathrm{~Hz}, 1 \mathrm{H}), 5.90-5.78(\mathrm{~m}$, $1 \mathrm{H}), 2.19(\mathrm{~s}, 3 \mathrm{H}) .{ }^{13} \mathrm{C} \mathrm{NMR}\left(101 \mathrm{MHz}, \mathrm{CDCl}_{3}\right) \delta 168.8,138.8,135.0,129.1$, 128.8, 127.0, 123.2 (q, $J=280.4 \mathrm{~Hz}), 117.3(\mathrm{q}, J=1.5 \mathrm{~Hz}), 71.1$ (q, $J=33.5 \mathrm{~Hz}), 20.6 .{ }^{19} \mathrm{~F}$ NMR $\left(377 \mathrm{MHz}, \mathrm{CDCl}_{3}\right) \delta-76.50\left(\mathrm{~d}, J=6.6 \mathrm{~Hz}\right.$ ). HRMS (EI) $\mathrm{m} / z$ : calcd for $\mathrm{C}_{12} \mathrm{H}_{11} \mathrm{~F}_{3} \mathrm{O}_{2}\left[\mathrm{M}^{+\bullet}\right]$ 244.0711, found: 244.0704 . 
(S)-4-Benzyl-1,3-diphenyl-4-((S,E)-4,4,4-trifluoro-1-phenylbut-2-en-1-yl)-1 $H$-pyrazol-5(4H)-one (4a)

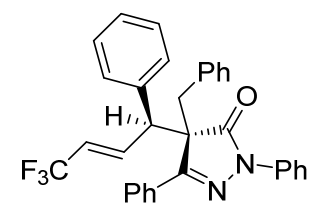

Column chromatography elution: petroleum ether/ethyl acetate $=50: 1(\mathrm{v} / \mathrm{v})$, colorless oil, $204.2 \mathrm{mg}, 80 \%$ yield, $>20: 1 \mathrm{dr}$, $94 \%$ ee.

$[\alpha]_{\mathrm{D}}^{20}=+67.08\left(\mathrm{c} 0.40, \mathrm{CH}_{2} \mathrm{Cl}_{2}\right)$.

${ }^{\mathrm{H}} \mathrm{NMR}\left(400 \mathrm{MHz}, \mathrm{CDCl}_{3}\right) \delta 7.90-7.82(\mathrm{~m}, 2 \mathrm{H}), 7.63-7.56(\mathrm{~m}, 1 \mathrm{H})$, $7.54-7.45(\mathrm{~m}, 3 \mathrm{H}), 7.42-7.39(\mathrm{~m}, 2 \mathrm{H}), 7.31-7.28(\mathrm{~m}, 2 \mathrm{H}), 7.15(\mathrm{t}, J=7.4 \mathrm{~Hz}, 1 \mathrm{H}), 7.12-$ $7.07(\mathrm{~m}, 1 \mathrm{H}), 7.07-6.96(\mathrm{~m}, 7 \mathrm{H}), 6.85(\mathrm{~d}, J=7.3 \mathrm{~Hz}, 2 \mathrm{H}), 5.92(\mathrm{dq}, J=15.6,6.2 \mathrm{~Hz}, 1 \mathrm{H}), 4.23$ $(\mathrm{d}, J=10.0 \mathrm{~Hz}, 1 \mathrm{H}), 3.60(\mathrm{~d}, J=13.6 \mathrm{~Hz}, 1 \mathrm{H}), 3.46(\mathrm{~d}, J=13.6 \mathrm{~Hz}, 1 \mathrm{H}) .{ }^{13} \mathrm{C}$ NMR $(101 \mathrm{MHz}$, $\left.\mathrm{CDCl}_{3}\right) \delta 174.0,157.3,137.1,137.0(\mathrm{q}, J=7.1 \mathrm{~Hz}), 136.5,134.2,131.7,130.4,129.3,128.9$, 128.7, 128.5, 128.2, 128.1, 127.8, 127.3, 126.7, 125.9, 122.7 (q, $J=270.7 \mathrm{~Hz}), 121.7$ (q, $J=33.9$ $\mathrm{Hz}), 120.4,63.6,53.5,41.5 .{ }^{19} \mathrm{~F} \mathrm{NMR}\left(377 \mathrm{MHz}, \mathrm{CDCl}_{3}\right) \delta-63.80(\mathrm{~d}, J=6.3 \mathrm{~Hz})$.

HPLC analysis: Daicel CHIRALPAK OD-H, $n$-hexane $/ i-\mathrm{PrOH}=95 / 5$, flow rate $=0.8 \mathrm{~mL} / \mathrm{min}, \lambda$ $=254 \mathrm{~nm}$, retention time: $t_{\text {minor }}=6.14 \mathrm{~min}, t_{\text {major }}=6.78 \mathrm{~min}$.

HRMS (ESI) $m / z$ : calcd for $\mathrm{C}_{32} \mathrm{H}_{26} \mathrm{~F}_{3} \mathrm{~N}_{2} \mathrm{O}[\mathrm{M}+\mathrm{H}]^{+}$511.1997, found: 511.1989.

(S)-4-Benzyl-1,3-diphenyl-4-((S,E)-4,4,4-trifluoro-1-(o-tolyl)but-2-en-1-yl)-1 $H$-pyrazol-5(4H)-on e (4b)

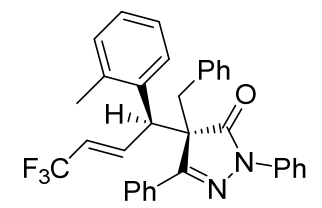

Column chromatography elution: petroleum ether/ethyl acetate $=50: 1(\mathrm{v} / \mathrm{v})$, colorless oil, $236.1 \mathrm{mg}, 90 \%$ yield, $>20: 1 \mathrm{dr}, 99 \%$ ee.

$[\alpha]_{\mathrm{D}}^{20}=+68.45\left(\mathrm{c} 0.50, \mathrm{CH}_{2} \mathrm{Cl}_{2}\right)$.

${ }^{1} \mathrm{H}$ NMR $\left(400 \mathrm{MHz}, \mathrm{CDCl}_{3}\right) \delta 7.94-7.83(\mathrm{~m}, 2 \mathrm{H}), 7.66-7.55(\mathrm{~m}, 1 \mathrm{H})$, $7.54-7.40(\mathrm{~m}, 5 \mathrm{H}), 7.32(\mathrm{t}, J=7.4 \mathrm{~Hz}, 2 \mathrm{H}), 7.17(\mathrm{t}, J=7.2 \mathrm{~Hz}, 1 \mathrm{H}), 7.09-6.93(\mathrm{~m}, 5 \mathrm{H}), 6.84(\mathrm{~d}$, $J=7.4 \mathrm{~Hz}, 2 \mathrm{H}), 6.76(\mathrm{~d}, J=7.4 \mathrm{~Hz}, 2 \mathrm{H}), 5.94-5.89(\mathrm{~m}, 1 \mathrm{H}), 4.22(\mathrm{~d}, J=9.9 \mathrm{~Hz}, 1 \mathrm{H}), 3.60(\mathrm{~d}, J$ $=13.5 \mathrm{~Hz}, 1 \mathrm{H}), 3.46(\mathrm{~d}, J=13.5 \mathrm{~Hz}, 1 \mathrm{H}), 2.16(\mathrm{~s}, 3 \mathrm{H}) .{ }^{13} \mathrm{C} \mathrm{NMR}\left(101 \mathrm{MHz}, \mathrm{CDCl}_{3}\right)($ one aromatic carbon missing) $\delta 174.1,157.5,137.8,137.2(\mathrm{q}, J=6.1 \mathrm{~Hz}), 137.13,137.11,134.3$, $133.5,131.7,130.4,129.3,129.2,128.9,128.7,128.2,127.7,127.3,126.7,125.9,122.8$ (q, $J=$ $271.7 \mathrm{~Hz}), 121.4(\mathrm{q}, J=33.8,1 \mathrm{H}), 120.4,63.7,53.2,41.6,21.0 .{ }^{19} \mathrm{~F} \mathrm{NMR}\left(377 \mathrm{MHz}, \mathrm{CDCl}_{3}\right) \delta$ $-63.81(\mathrm{~d}, J=6.3 \mathrm{~Hz})$.

HPLC analysis: Daicel CHIRALPAK AD-H, $n$-hexane $/ i-\mathrm{PrOH}=95 / 5$, flow rate $=0.8 \mathrm{~mL} / \mathrm{min}, \lambda$ $=254 \mathrm{~nm}$, retention time: $t_{\text {minor }}=5.19 \mathrm{~min}, t_{\text {major }}=6.45 \mathrm{~min}$.

HRMS (ESI) $m / z$ : calcd for $\mathrm{C}_{33} \mathrm{H}_{28} \mathrm{~F}_{3} \mathrm{~N}_{2} \mathrm{O}[\mathrm{M}+\mathrm{H}]^{+}$525.2154, found: 525.2142 .

(S)-4-Benzyl-1,3-diphenyl-4-((S,E)-4,4,4-trifluoro-1-( $m$-tolyl)but-2-en-1-yl)-1 $H$-pyrazol-5(4H)-on $\mathrm{e}(\mathbf{4 c})$

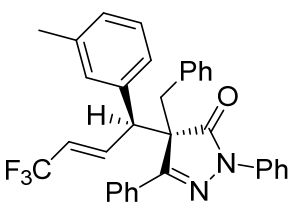

Column chromatography elution: petroleum ether/ethyl acetate $=50: 1(\mathrm{v} / \mathrm{v})$, colorless oil, $215.1 \mathrm{mg}, 82 \%$ yield, $>20: 1 \mathrm{dr}, 94 \%$ ee. $[\alpha]_{\mathrm{D}}^{20}=+83.61\left(\mathrm{c} 0.77, \mathrm{CH}_{2} \mathrm{Cl}_{2}\right)$.

${ }^{1} \mathrm{H}$ NMR $\left(400 \mathrm{MHz}, \mathrm{CDCl}_{3}\right) \delta 7.90-7.84(\mathrm{~m}, 2 \mathrm{H}), 7.65-7.55(\mathrm{~m}, 1 \mathrm{H})$, 
$7.55-7.49(\mathrm{~m}, 3 \mathrm{H}), 7.49-7.44(\mathrm{~m}, 2 \mathrm{H}), 7.37-7.30(\mathrm{~m}, 2 \mathrm{H}), 7.22-7.16(\mathrm{~m}, 1 \mathrm{H}), 7.10-7.02(\mathrm{~m}$, $5 \mathrm{H}), 6.96-6.89(\mathrm{~m}, 2 \mathrm{H}), 6.70-6.63(\mathrm{~m}, 2 \mathrm{H}), 5.94(\mathrm{dq}, J=15.6,6.2 \mathrm{~Hz}, 1 \mathrm{H}), 4.19(\mathrm{~d}, J=10.0$ $\mathrm{Hz}, 1 \mathrm{H}), 3.63(\mathrm{~d}, J=13.6 \mathrm{~Hz}, 1 \mathrm{H}), 3.49(\mathrm{~d}, J=13.6 \mathrm{~Hz}, 1 \mathrm{H}), 2.03(\mathrm{~s}, 3 \mathrm{H}) .{ }^{13} \mathrm{C} \mathrm{NMR}(101 \mathrm{MHz}$, $\left.\mathrm{CDCl}_{3}\right) \delta 174.1,157.5,138.2,137.1,137.0(\mathrm{q}, J=6.5 \mathrm{~Hz}), 136.3,134.3,131.8,130.4,129.3$, $128.9,128.8,128.7,128.5,128.3,128.2,127.3,126.8,125.9,124.9,122.7$ (q, $J=270.7 \mathrm{~Hz}$ ), $121.5(\mathrm{q}, J=33.8 \mathrm{~Hz}), 120.3,63.7,53.6,41.4,21.1 .{ }^{19} \mathrm{~F}$ NMR $\left(377 \mathrm{MHz}, \mathrm{CDCl}_{3}\right) \delta-63.75(\mathrm{~d}, J=$ $6.3 \mathrm{~Hz})$.

HPLC analysis: Daicel CHIRALPAK AD-H, $n$-hexane $/ i-\mathrm{PrOH}=50 / 1$, flow rate $=0.8 \mathrm{~mL} / \mathrm{min}, \lambda$ $=254 \mathrm{~nm}$, retention time: $t_{\text {major }}=8.77 \mathrm{~min}, t_{\text {minor }}=10.56 \mathrm{~min}$.

HRMS (ESI) $m / z$ : calcd for $\mathrm{C}_{33} \mathrm{H}_{28} \mathrm{~F}_{3} \mathrm{~N}_{2} \mathrm{O}[\mathrm{M}+\mathrm{H}]^{+}$525.2154, found: 525.2143 .

(S)-4-Benzyl-1,3-diphenyl-4-((S,E)-4,4,4-trifluoro-1-( $p$-tolyl)but-2-en-1-yl)-1 $H$-pyrazol-5(4H)-on e (4d)

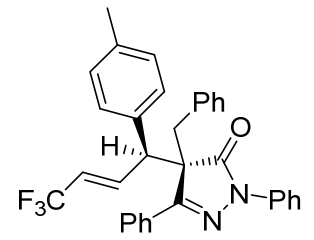

Column chromatography elution: petroleum ether/ethyl acetate $=50: 1(\mathrm{v} / \mathrm{v})$, colorless oil, $212.5 \mathrm{mg}, 81 \%$ yield, $>20: 1 \mathrm{dr}, 91 \%$ ee.

$[\alpha]_{\mathrm{D}}^{20}=+62.83\left(\mathrm{c} 0.67, \mathrm{CH}_{2} \mathrm{Cl}_{2}\right)$.

${ }^{1} \mathrm{H}$ NMR $\left(400 \mathrm{MHz}, \mathrm{CDCl}_{3}\right) \delta 7.91-7.84(\mathrm{~m}, 2 \mathrm{H}), 7.62-7.55(\mathrm{~m}, 1 \mathrm{H})$, $7.53-7.46(\mathrm{~m}, 3 \mathrm{H}), 7.43(\mathrm{dd}, J=8.6,1.0 \mathrm{~Hz}, 2 \mathrm{H}), 7.36-7.28(\mathrm{~m}, 2 \mathrm{H})$, $7.19-7.15(\mathrm{~m}, 1 \mathrm{H}), 7.10-6.95(\mathrm{~m}, 5 \mathrm{H}), 6.84(\mathrm{~d}, J=8.0 \mathrm{~Hz}, 2 \mathrm{H}), 6.75(\mathrm{~d}, J=8.2 \mathrm{~Hz}, 2 \mathrm{H}), 5.91$ $(\mathrm{dq}, J=15.6,6.2 \mathrm{~Hz}, 1 \mathrm{H}), 4.21(\mathrm{~d}, J=10.0 \mathrm{~Hz}, 1 \mathrm{H}), 3.59(\mathrm{~d}, J=13.5 \mathrm{~Hz}, 1 \mathrm{H}), 3.45(\mathrm{~d}, J=13.5$ $\mathrm{Hz}, 1 \mathrm{H}), 2.17$ (s, 3H). ${ }^{13} \mathrm{C} \mathrm{NMR}\left(101 \mathrm{MHz}, \mathrm{CDCl}_{3}\right) \delta 174.1,157.5,137.8,137.2$ (q, $\left.J=6.1 \mathrm{~Hz}\right)$, 137.1, 134.2, 133.5, 131.7, 130.4, 129.3, 129.2, 128.9, 128.7, 128.2, 127.7, 127.3, 126.7, 125.9, 122.7 (q, $J=270.7 \mathrm{~Hz}), 121.4(\mathrm{q}, J=33.9 \mathrm{~Hz}), 120.4,63.7,53.2,41.6,21.0 .{ }^{19} \mathrm{~F}$ NMR $(377 \mathrm{MHz}$, $\left.\mathrm{CDCl}_{3}\right) \delta-63.80(\mathrm{~d}, J=6.2 \mathrm{~Hz})$.

HPLC analysis: Daicel CHIRALPAK AD-H, $n$-hexane $/ i-\mathrm{PrOH}=50 / 1$, flow rate $=0.8 \mathrm{~mL} / \mathrm{min}, \lambda$ $=254 \mathrm{~nm}$, retention time: $t_{\text {major }}=9.57 \mathrm{~min}, t_{\text {minor }}=10.79 \mathrm{~min}$.

HRMS (ESI) $m / z$ : calcd for $\mathrm{C}_{33} \mathrm{H}_{28} \mathrm{~F}_{3} \mathrm{~N}_{2} \mathrm{O}[\mathrm{M}+\mathrm{H}]^{+}$525.2154, found: 525.2149 .

(S)-4-Benzyl-1,3-diphenyl-4-((S,E)-4,4,4-trifluoro-1-(4-isopropylphenyl)but-2-en-1-yl)-1 $H$-pyrazo $1-5(4 H)$-one (4e)

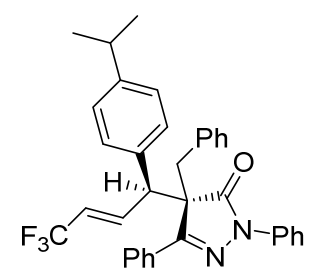

Column chromatography elution: petroleum ether/ethyl acetate $=50: 1(\mathrm{v} / \mathrm{v})$, colorless oil, $229.3 \mathrm{mg}, 83 \%$ yield, $>20: 1 \mathrm{dr}, 99 \%$ ee.

$[\alpha]_{\mathrm{D}}^{20}=+59.70\left(\mathrm{c} 0.41, \mathrm{CH}_{2} \mathrm{Cl}_{2}\right)$.

${ }^{1} \mathrm{H}$ NMR $\left(400 \mathrm{MHz}, \mathrm{CDCl}_{3}\right) \delta 7.78(\mathrm{~d}, J=4.6 \mathrm{~Hz}, 2 \mathrm{H}), 7.55-7.45(\mathrm{~m}, 1 \mathrm{H})$, $7.44-7.37(\mathrm{~m}, 3 \mathrm{H}), 7.27(\mathrm{~d}, J=8.0 \mathrm{~Hz}, 2 \mathrm{H}), 7.20(\mathrm{t}, J=7.6 \mathrm{~Hz}, 2 \mathrm{H}), 7.07$ (t, $J=7.2 \mathrm{~Hz}, 1 \mathrm{H}), 6.99-6.85(\mathrm{~m}, 5 \mathrm{H}), 6.79(\mathrm{~d}, J=7.7 \mathrm{~Hz}, 2 \mathrm{H}), 6.70(\mathrm{~d}, J=7.7 \mathrm{~Hz}, 2 \mathrm{H}), 5.83(\mathrm{dq}$, $J=12.3,6.0 \mathrm{~Hz}, 1 \mathrm{H}), 4.14(\mathrm{~d}, J=10.1 \mathrm{~Hz}, 1 \mathrm{H}), 3.50(\mathrm{~d}, J=13.5 \mathrm{~Hz}, 1 \mathrm{H}), 3.35(\mathrm{~d}, J=13.5 \mathrm{~Hz}$, $1 \mathrm{H}), 2.63$ (heptet, $J=6.9 \mathrm{~Hz}, 1 \mathrm{H}), 1.00(\mathrm{~d}, J=6.9 \mathrm{~Hz}, 6 \mathrm{H}) .{ }^{13} \mathrm{C} \mathrm{NMR}\left(101 \mathrm{MHz}, \mathrm{CDCl}_{3}\right) \delta 174.1$, 157.4, 148.7, 137.08 (q, $J=7.1 \mathrm{~Hz}), 137.07,134.3,133.6,131.8,130.4,129.3,128.9,128.6$, 
$128.2,127.8,127.3,126.7,126.4,125.9,122.7$ (q, $J=270.7 \mathrm{~Hz}), 121.4(\mathrm{q}, J=34.0 \mathrm{~Hz}), 120.5$, 63.8, 53.1, 41.3, 33.6, 23.9, 23.7. ${ }^{19} \mathrm{~F}$ NMR $\left(377 \mathrm{MHz}, \mathrm{CDCl}_{3}\right) \delta-63.73(\mathrm{~d}, J=6.0 \mathrm{~Hz})$.

HPLC analysis: Daicel CHIRALPAK AD-H, $n$-hexane $/ i-\mathrm{PrOH}=50 / 1$, flow rate $=0.8 \mathrm{~mL} / \mathrm{min}, \lambda$ $=254 \mathrm{~nm}$, retention time: $t_{\text {major }}=7.04 \mathrm{~min}, t_{\text {minor }}=8.75 \mathrm{~min}$.

HRMS (ESI) $m / z$ : calcd for $\mathrm{C}_{35} \mathrm{H}_{32} \mathrm{~F}_{3} \mathrm{~N}_{2} \mathrm{O}[\mathrm{M}+\mathrm{H}]^{+}$553.2467, found: 553.2457.

(S)-4-Benzyl-1,3-diphenyl-4-((S,E)-4,4,4-trifluoro-1-(2-methoxyphenyl)but-2-en-1-yl)-1H-pyrazol $-5(4 H)$-one $(\mathbf{4 f})$

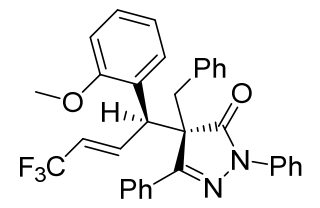

Column chromatography elution: petroleum ether/ethyl acetate $=50: 1(\mathrm{v} / \mathrm{v})$, colorless oil, $221.6 \mathrm{mg}, 82 \%$ yield, $>20: 1 \mathrm{dr}, 97 \%$ ee.

$[\alpha]_{\mathrm{D}}^{20}=+90.33\left(\mathrm{c} 0.33, \mathrm{CH}_{2} \mathrm{Cl}_{2}\right)$.

${ }^{1} \mathrm{H}$ NMR $\left(400 \mathrm{MHz}, \mathrm{CDCl}_{3}\right) \delta 7.88-7.81(\mathrm{~m}, 2 \mathrm{H}), 7.55-7.46(\mathrm{~m}, 3 \mathrm{H})$, $7.45-7.39(\mathrm{~m}, 3 \mathrm{H}), 7.31(\mathrm{t}, J=7.9 \mathrm{~Hz}, 2 \mathrm{H}), 7.19-7.12(\mathrm{~m}, 2 \mathrm{H}), 7.07-6.97(\mathrm{~m}, 6 \mathrm{H}), 6.66(\mathrm{t}, J=$ $7.6 \mathrm{~Hz}, 1 \mathrm{H}), 6.53(\mathrm{~d}, J=8.1 \mathrm{~Hz}, 1 \mathrm{H}), 5.96-5.84(\mathrm{~m}, 1 \mathrm{H}), 4.95(\mathrm{~d}, J=9.8 \mathrm{~Hz}, 1 \mathrm{H}), 3.63(\mathrm{~d}, J=$ $13.5 \mathrm{~Hz}, 1 \mathrm{H}), 3.50$ (d, $J=13.5 \mathrm{~Hz}, 1 \mathrm{H}), 3.14(\mathrm{~s}, 3 \mathrm{H}) .{ }^{13} \mathrm{C} \mathrm{NMR}\left(101 \mathrm{MHz}, \mathrm{CDCl}_{3}\right) \delta 174.4,157.8$, 155.8, $137.4(\mathrm{q}, J=6.5 \mathrm{~Hz}), 137.2,134.3,131.7,129.9,129.4,128.9,128.7,128.3,128.1,128.0$, $127.2,126.9,125.7,125.1,122.8(\mathrm{q}, J=269.9 \mathrm{~Hz}), 121.0(\mathrm{q}, J=33.7 \mathrm{~Hz}), 120.5,120.2,110.2$, 63.3, 54.3, 44.6, 41.9. ${ }^{19} \mathrm{~F}$ NMR $\left(377 \mathrm{MHz}, \mathrm{CDCl}_{3}\right) \delta-63.66(\mathrm{~d}, J=6.2 \mathrm{~Hz})$.

HPLC analysis: Daicel CHIRALPAK AD-H, $n$-hexane $/ i-\mathrm{PrOH}=50 / 1$, flow rate $=0.8 \mathrm{~mL} / \mathrm{min}, \lambda$ $=254 \mathrm{~nm}$, retention time: $t_{\text {major }}=7.28 \mathrm{~min}, t_{\text {minor }}=14.43 \mathrm{~min}$.

HRMS (ESI) $m / z$ : calcd for $\mathrm{C}_{33} \mathrm{H}_{28} \mathrm{~F}_{3} \mathrm{~N}_{2} \mathrm{O}_{2}[\mathrm{M}+\mathrm{H}]^{+}$541.2103, found: 541.2094.

(S)-4-Benzyl-1,3-diphenyl-4-((S,E)-4,4,4-trifluoro-1-(naphthalen-2-yl)but-2-en-1-yl)-1H-pyrazol$5(4 H)$-one $(\mathbf{4 g})$

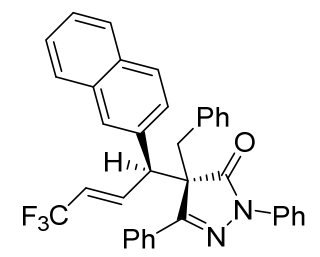

Column chromatography elution: petroleum ether/ethyl acetate $=50: 1(\mathrm{v} / \mathrm{v})$, colorless oil, $246.7 \mathrm{mg}, 88 \%$ yield, $>20: 1 \mathrm{dr}, 94 \%$ ee.

$[\alpha]_{\mathrm{D}}^{20}=+83.53\left(\mathrm{c} 0.16, \mathrm{CH}_{2} \mathrm{Cl}_{2}\right)$.

${ }^{1} \mathrm{H}$ NMR $\left(400 \mathrm{MHz}, \mathrm{CDCl}_{3}\right) \delta 7.83(\mathrm{~d}, J=6.9 \mathrm{~Hz}, 2 \mathrm{H}), 7.77-7.66(\mathrm{~m}, 1 \mathrm{H})$, $7.63(\mathrm{~d}, J=7.6 \mathrm{~Hz}, 1 \mathrm{H}), 7.55-7.44(\mathrm{~m}, 4 \mathrm{H}), 7.43-7.28(\mathrm{~m}, 5 \mathrm{H}), 7.28-$ $7.19(\mathrm{~m}, 3 \mathrm{H}), 7.12(\mathrm{t}, J=7.3 \mathrm{~Hz}, 1 \mathrm{H}), 7.03(\mathrm{~d}, J=3.6 \mathrm{~Hz}, 6 \mathrm{H}), 5.95(\mathrm{dq}, J=15.6,6.1 \mathrm{~Hz}, 1 \mathrm{H})$, $4.39(\mathrm{~d}, J=9.6 \mathrm{~Hz}, 1 \mathrm{H}), 3.67(\mathrm{~d}, J=13.5,1 \mathrm{H}), 3.54(\mathrm{~d}, J=13.5,1 \mathrm{H}) .{ }^{13} \mathrm{C}$ NMR $(101 \mathrm{MHz}$, $\mathrm{CDCl}_{3}$ ) (one aromatic carbon missing) $\delta 174.1,157.5,137.0,136.9(\mathrm{q}, J=6.1 \mathrm{~Hz}), 134.1,133.9$, 133.0, 132.8, 131.8, 130.5, 129.3, 128.9, 128.7, 128.33, 128.31, 127.8, 127.5, 127.4, 127.3, 126.8, $126.3,126.0,125.3,122.7$ (q, $J=270.7 \mathrm{~Hz}), 121.82(\mathrm{q}, J=33.8 \mathrm{~Hz}), 120.4,63.7,53.7,41.7 .{ }^{19} \mathrm{~F}$ $\operatorname{NMR}\left(377 \mathrm{MHz}, \mathrm{CDCl}_{3}\right) \delta-63.83(\mathrm{~d}, J=5.9 \mathrm{~Hz})$.

HPLC analysis: Daicel CHIRALPAK AD-H, $n$-hexane $/ i-\mathrm{PrOH}=100 / 1$, flow rate $=0.8 \mathrm{~mL} / \mathrm{min}, \lambda$ $=254 \mathrm{~nm}$, retention time: $t_{\text {major }}=16.38 \mathrm{~min}, t_{\text {minor }}=21.72 \mathrm{~min}$.

HRMS (ESI) $m / z$ : calcd for $\mathrm{C}_{36} \mathrm{H}_{28} \mathrm{~F}_{3} \mathrm{~N}_{2} \mathrm{O}[\mathrm{M}+\mathrm{H}]^{+}$561.2154, found: 561.2144 . 
(S)-4-((S,E)-1-([1,1'-Biphenyl]-4-yl)-4,4,4-trifluorobut-2-en-1-yl)-4-benzyl-1,3-diphenyl-1H-pyra zol-5(4H)-one (4h)

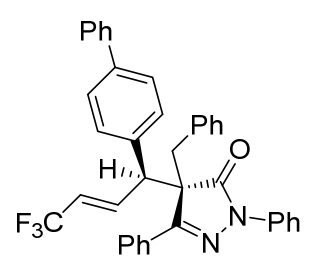

Column chromatography elution: petroleum ether/ethyl acetate $=50: 1(\mathrm{v} / \mathrm{v})$, colorless oil, $249.3 \mathrm{mg}, 85 \%$ yield, $>20: 1 \mathrm{dr}, 94 \%$ ee.

$[\alpha]_{\mathrm{D}}{ }^{20}=+53.86\left(\mathrm{c} 0.44, \mathrm{CH}_{2} \mathrm{Cl}_{2}\right)$.

${ }^{1} \mathrm{H}$ NMR $\left(400 \mathrm{MHz}, \mathrm{CDCl}_{3}\right) \delta 7.89(\mathrm{~d}, J=5.4 \mathrm{~Hz}, 2 \mathrm{H}), 7.68-7.58(\mathrm{~m}$, $1 \mathrm{H}), 7.54-7.49(\mathrm{~m}, 3 \mathrm{H}), 7.45-7.40(\mathrm{~m}, 4 \mathrm{H}), 7.39-7.23(\mathrm{~m}, 8 \mathrm{H}), 7.16(\mathrm{t}$, $J=7.3 \mathrm{~Hz}, 1 \mathrm{H}), 7.10-6.98(\mathrm{~m}, 5 \mathrm{H}), 6.93(\mathrm{~d}, J=7.6 \mathrm{~Hz}, 2 \mathrm{H}), 6.03-5.90$ $(\mathrm{m}, 1 \mathrm{H}), 4.29(\mathrm{~d}, J=9.9 \mathrm{~Hz}, 1 \mathrm{H}), 3.62(\mathrm{~d}, J=13.5 \mathrm{~Hz}, 1 \mathrm{H}), 3.49(\mathrm{~d}, J=13.5 \mathrm{~Hz}, 1 \mathrm{H}) .{ }^{13} \mathrm{C}$ NMR $\left(101 \mathrm{MHz}, \mathrm{CDCl}_{3}\right)$ (one aromatic carbon missing) $\delta 174.0,157.3,140.8,140.2,137.0,136.9$ (q, $J$ $=6.7 \mathrm{~Hz}), 135.5,134.2,131.7,130.5,129.3,129.0,128.74,128.73,128.3,127.5,127.4,127.1$, 127.0, 126.7, 126.0, 122.7 (q, $J=270.7 \mathrm{~Hz}), 121.7$ (q, $J=34.0 \mathrm{~Hz}), 120.4,63.7,53.2,41.5 .{ }^{19} \mathrm{~F}$ $\operatorname{NMR}\left(377 \mathrm{MHz}, \mathrm{CDCl}_{3}\right) \delta-63.73(\mathrm{~d}, J=6.1 \mathrm{~Hz})$.

HPLC analysis: Daicel CHIRALPAK AD-H, $n$-hexane $/ i-\mathrm{PrOH}=50 / 1$, flow rate $=0.8 \mathrm{~mL} / \mathrm{min}, \lambda$ $=254 \mathrm{~nm}$, retention time: $t_{\text {major }}=9.53 \mathrm{~min}, t_{\text {minor }}=11.93 \mathrm{~min}$.

HRMS (ESI) $m / z$ : calcd for $\mathrm{C}_{38} \mathrm{H}_{30} \mathrm{~F}_{3} \mathrm{~N}_{2} \mathrm{O}[\mathrm{M}+\mathrm{H}]^{+}$587.2310, found: 587.2309.

(S)-4-Benzyl-1,3-diphenyl-4-((S,E)-4,4,4-trifluoro-1-(4-fluorophenyl)but-2-en-1-yl)-1H-pyrazol-5 (4H)-one (4i)

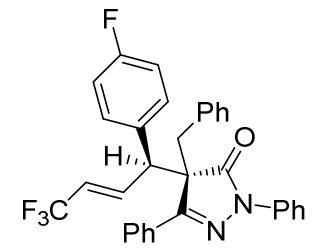

Column chromatography elution: petroleum ether/ethyl acetate $=50: 1(\mathrm{v} / \mathrm{v})$, colorless oil, $222.0 \mathrm{mg}, 84 \%$ yield, $>20: 1 \mathrm{dr}, 95 \%$ ee.

$[\alpha]_{\mathrm{D}}^{20}=+62.40\left(\mathrm{c} 0.53, \mathrm{CH}_{2} \mathrm{Cl}_{2}\right)$.

${ }^{1} \mathrm{H}$ NMR $\left(400 \mathrm{MHz}, \mathrm{CDCl}_{3}\right) \delta 7.89-7.86(\mathrm{~m}, 2 \mathrm{H}), 7.64-7.54(\mathrm{~m}, 1 \mathrm{H})$, $7.54-7.47(\mathrm{~m}, 3 \mathrm{H}), 7.42(\mathrm{~d}, J=8.1 \mathrm{~Hz}, 2 \mathrm{H}), 7.31(\mathrm{dd}, J=10.7,5.1 \mathrm{~Hz}$, 2H), $7.18-7.14(\mathrm{~m}, 1 \mathrm{H}), 7.07-6.96(\mathrm{~m}, 5 \mathrm{H}), 6.85-6.79(\mathrm{~m}, 2 \mathrm{H}), 6.76-6.68(\mathrm{~m}, 2 \mathrm{H}), 5.91(\mathrm{dq}$, $J=15.6,6.1 \mathrm{~Hz}, 1 \mathrm{H}), 4.23(\mathrm{~d}, J=9.9 \mathrm{~Hz}, 1 \mathrm{H}), 3.58(\mathrm{~d}, J=13.5 \mathrm{~Hz}, 1 \mathrm{H}), 3.45(\mathrm{~d}, J=13.5 \mathrm{~Hz}$, 1H). ${ }^{13} \mathrm{C}$ NMR $\left(101 \mathrm{MHz}, \mathrm{CDCl}_{3}\right) \delta 173.9,162.2(\mathrm{~d}, J=247.5 \mathrm{~Hz}), 157.2,136.9,136.6$ (q, $J=6.6$ $\mathrm{Hz}), 134.0,132.3,131.5,130.5,129.5(\mathrm{~d}, J=8.1 \mathrm{~Hz}), 129.2,129.0,128.8,128.3,127.4,126.6$, 126.0, 122.6 (q, $J=270.7 \mathrm{~Hz}), 121.85$ (q, $J=34.0 \mathrm{~Hz}), 120.2,115.4$ (d, $J=21.5 \mathrm{~Hz}), 63.6,52.6$, 41.4. ${ }^{19} \mathrm{~F}$ NMR $\left(377 \mathrm{MHz}, \mathrm{CDCl}_{3}\right) \delta-63.85(\mathrm{~d}, J=6.0 \mathrm{~Hz}, 3 \mathrm{~F}),-113.49--113.56(\mathrm{~m}, 1 \mathrm{~F})$.

HPLC analysis: Daicel CHIRALPAK AD-H, $n$-hexane $/ i-\mathrm{PrOH}=100 / 1$, flow rate $=0.8 \mathrm{~mL} / \mathrm{min}, \lambda$ $=254 \mathrm{~nm}$, retention time: $t_{\text {major }}=14.74 \mathrm{~min}, t_{\text {minor }}=18.59 \mathrm{~min}$.

HRMS (ESI) $m / z$ : calcd for $\mathrm{C}_{32} \mathrm{H}_{25} \mathrm{~F}_{4} \mathrm{~N}_{2} \mathrm{O}[\mathrm{M}+\mathrm{H}]^{+}$529.1903, found: 529.1896 .

(S)-4-Benzyl-4-((S,E)-1-(3-chlorophenyl)-4,4,4-trifluorobut-2-en-1-yl)-1,3-diphenyl-1 $H$-pyrazol-5 $(4 H)$-one $(\mathbf{4 j})$

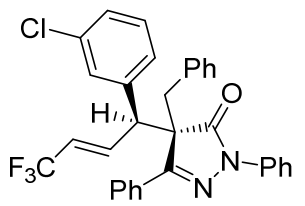

Column chromatography elution: petroleum ether/ethyl acetate $=50: 1(\mathrm{v} / \mathrm{v})$, white solid, mp $54-55{ }^{\circ} \mathrm{C}, 234.3 \mathrm{mg}, 86 \%$ yield, > 20:1 dr, $92 \%$ ee. 
$[\alpha]_{\mathrm{D}}^{20}=+75.27\left(\mathrm{c} 0.64, \mathrm{CH}_{2} \mathrm{Cl}_{2}\right)$.

${ }^{1} \mathrm{H}$ NMR (400 MHz, $\left.\mathrm{CDCl}_{3}\right) \delta 7.85$ (dd, $\left.J=7.2,2.4 \mathrm{~Hz}, 2 \mathrm{H}\right), 7.61-7.47$ (m, 4H), 7.41 (d, $J=8.3$ $\mathrm{Hz}, 2 \mathrm{H}), 7.37-7.28(\mathrm{~m}, 2 \mathrm{H}), 7.22-7.14(\mathrm{~m}, 1 \mathrm{H}), 7.09-6.95(\mathrm{~m}, 7 \mathrm{H}), 6.81-6.74(\mathrm{~m}, 2 \mathrm{H}), 5.93$ (dq, $J=15.6,6.1 \mathrm{~Hz}, 1 \mathrm{H}), 4.17(\mathrm{~d}, J=10.0 \mathrm{~Hz}, 1 \mathrm{H}), 3.58(\mathrm{~d}, J=13.6 \mathrm{~Hz}, 1 \mathrm{H}), 3.46(\mathrm{~d}, J=13.6$ $\mathrm{Hz}, 1 \mathrm{H}) .{ }^{13} \mathrm{C}$ NMR $\left(101 \mathrm{MHz}, \mathrm{CDCl}_{3}\right) \delta 173.8,157.2,138.4,136.8,136.1$ (q, $\left.J=7.1 \mathrm{~Hz}\right), 134.3$, 133.9, 131.5, 130.6, 129.8, 129.2, 129.1, 128.8, 128.3, 128.28, 128.22, 127.5, 126.7, 126.2, 125.8, $122.5(\mathrm{q}, J=271.7 \mathrm{~Hz}), 122.2(\mathrm{q}, J=34.0 \mathrm{~Hz}), 120.5,63.4,53.0,41.4 .{ }^{19} \mathrm{~F}$ NMR $(377 \mathrm{MHz}$, $\left.\mathrm{CDCl}_{3}\right) \delta-63.93(\mathrm{~d}, J=5.9 \mathrm{~Hz})$.

HPLC analysis: Daicel CHIRALPAK AD-H, $n$-hexane $/ i-\mathrm{PrOH}=95 / 5$, flow rate $=0.8 \mathrm{~mL} / \mathrm{min}, \lambda$ $=254 \mathrm{~nm}$, retention time: $t_{\text {major }}=7.08 \mathrm{~min}, t_{\text {minor }}=7.93 \mathrm{~min}$.

HRMS (ESI) $m / z$ : calcd for $\mathrm{C}_{32} \mathrm{H}_{25} \mathrm{ClF}_{3} \mathrm{~N}_{2} \mathrm{O}[\mathrm{M}+\mathrm{H}]^{+}$545.1608, found: 545.1599.

(S)-4-Benzyl-4-((S,E)-1-(4-bromophenyl)-4,4,4-trifluorobut-2-en-1-yl)-1,3-diphenyl-1 $H$-pyrazol-5 (4H)-one (4k)

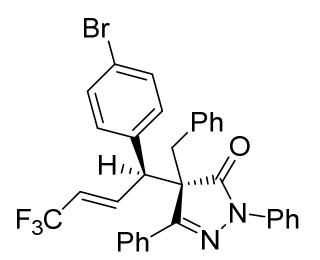

Column chromatography elution: petroleum ether/ethyl acetate $=50: 1(\mathrm{v} / \mathrm{v})$, white solid, mp $71-72{ }^{\circ} \mathrm{C}, 232.8 \mathrm{mg}, 79 \%$ yield, $>20: 1 \mathrm{dr}, 94 \%$ ee. $[\alpha]_{\mathrm{D}}{ }^{20}=+61.62\left(\mathrm{c} 0.56, \mathrm{CH}_{2} \mathrm{Cl}_{2}\right)$.

${ }^{1} \mathrm{H}$ NMR $\left(400 \mathrm{MHz}, \mathrm{CDCl}_{3}\right) \delta 7.88-7.84(\mathrm{~m}, 2 \mathrm{H}), 7.62-7.54(\mathrm{~m}, 1 \mathrm{H})$, $7.54-7.46(\mathrm{~m}, 3 \mathrm{H}), 7.45-7.39(\mathrm{~m}, 2 \mathrm{H}), 7.35-7.27(\mathrm{~m}, 2 \mathrm{H}), 7.21-7.13$ (m, 3H), $7.07-6.95(\mathrm{~m}, 5 \mathrm{H}), 6.75-6.69(\mathrm{~m}, 2 \mathrm{H}), 5.91(\mathrm{dq}, J=15.6,6.1 \mathrm{~Hz}, 1 \mathrm{H}), 4.20(\mathrm{~d}, J=$ $10.0 \mathrm{~Hz}, 1 \mathrm{H}), 3.57(\mathrm{~d}, J=13.5 \mathrm{~Hz}, 1 \mathrm{H}), 3.45(\mathrm{~d}, J=13.5 \mathrm{~Hz}, 1 \mathrm{H}) .{ }^{13} \mathrm{C} \mathrm{NMR}\left(101 \mathrm{MHz}, \mathrm{CDCl}_{3}\right) \delta$ 173.8, 157.1, 136.9, 136.3 (q, $J=6.5 \mathrm{~Hz}), 135.6,133.9,131.6,131.4,130.6,129.5,129.2,129.1$, $128.8,128.3,127.4,126.6,126.1,122.5$ (q, $J=270.7 \mathrm{~Hz}), 122.2,122.1$ (q, $J=34.3 \mathrm{~Hz}), 120.3$, 63.4, 52.8, 41.5. $\left.{ }^{19} \mathrm{~F} \mathrm{NMR} \mathrm{(377} \mathrm{MHz,} \mathrm{CDCl}_{3}\right) \delta-63.93(\mathrm{~d}, J=6.1 \mathrm{~Hz})$.

HPLC analysis: Daicel CHIRALPAK AD-H, $n$-hexane $/ i$-PrOH $=50 / 1$, flow rate $=0.8 \mathrm{~mL} / \mathrm{min}, \lambda$ $=254 \mathrm{~nm}$, retention time: $t_{\text {major }}=10.38 \mathrm{~min}, t_{\text {minor }}=12.90 \mathrm{~min}$.

HRMS (ESI) $m / z$ : calcd for $\mathrm{C}_{32} \mathrm{H}_{25} \mathrm{BrF}_{3} \mathrm{~N}_{2} \mathrm{O}[\mathrm{M}+\mathrm{H}]^{+}$589.1102, found: 589.1094 .

(S)-4-Benzyl-1,3-diphenyl-4-((S,E)-4,4,4-trifluoro-1-(3-(trifluoromethyl)phenyl)but-2-en-1-yl)-1H -pyrazol-5(4H)-one (4I)

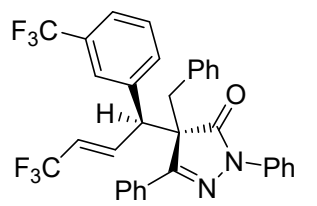

Column chromatography elution: petroleum ether/ethyl acetate $=50: 1(\mathrm{v} / \mathrm{v})$, colorless oil, $245.9 \mathrm{mg}, 85 \%$ yield, $>20: 1 \mathrm{dr}, 96 \%$ ee.

$[\alpha]_{\mathrm{D}}{ }^{20}=+94.57\left(\mathrm{c} 0.41, \mathrm{CH}_{2} \mathrm{Cl}_{2}\right)$.

${ }^{1} \mathrm{H}$ NMR $\left(400 \mathrm{MHz}, \mathrm{CDCl}_{3}\right) \delta 7.84(\mathrm{~d}, J=5.5 \mathrm{~Hz}, 2 \mathrm{H}), 7.65-7.57(\mathrm{~m}, 1 \mathrm{H})$, $7.55-7.46(\mathrm{~m}, 3 \mathrm{H}), 7.44-7.33(\mathrm{~m}, 3 \mathrm{H}), 7.32-7.26(\mathrm{~m}, 2 \mathrm{H}), 7.20-7.11(\mathrm{~m}, 3 \mathrm{H}), 7.09-6.98(\mathrm{~m}$, $5 \mathrm{H}), 6.97-6.91(\mathrm{~m}, 1 \mathrm{H}), 5.98-5.93(\mathrm{~m}, 1 \mathrm{H}), 4.26(\mathrm{~d}, J=9.8 \mathrm{~Hz}, 1 \mathrm{H}), 3.62(\mathrm{~d}, J=13.6 \mathrm{~Hz}, 1 \mathrm{H})$, $3.50(\mathrm{~d}, J=13.6 \mathrm{~Hz}, 1 \mathrm{H}) .{ }^{13} \mathrm{C} \mathrm{NMR}\left(101 \mathrm{MHz}, \mathrm{CDCl}_{3}\right) \delta 173.6,157.2,137.5,136.8,135.9(\mathrm{q}, J=$ $6.5 \mathrm{~Hz}), 133.8,131.3,130.9,130.8(\mathrm{q}, J=32.3 \mathrm{~Hz}), 130.7,129.2,129.1,129.0,128.7,128.3$, 127.5, 126.6, 126.1, 125.1 (q, $J=4.0 \mathrm{~Hz}), 124.9$ (q, $J=4.0 \mathrm{~Hz}), 123.5$ (q, $J=272.7 \mathrm{~Hz}), 122.5(\mathrm{q}$, $J=34.2 \mathrm{~Hz}, 1 \mathrm{H}), 122.4(\mathrm{q}, J=270.7 \mathrm{~Hz}), 120.2,63.4,53.1,41.5 .{ }^{19} \mathrm{~F} \mathrm{NMR}\left(377 \mathrm{MHz}, \mathrm{CDCl}_{3}\right) \delta$ 
$-62.92(\mathrm{~s}, 3 \mathrm{~F}),-63.94(\mathrm{~d}, J=5.9 \mathrm{~Hz}, 3 \mathrm{~F})$.

HPLC analysis: Daicel CHIRALPAK AD-H, $n$-hexane $/ i-\mathrm{PrOH}=50 / 1$, flow rate $=0.8 \mathrm{~mL} / \mathrm{min}, \lambda$ $=254 \mathrm{~nm}$, retention time: $t_{\text {major }}=7.74 \mathrm{~min}, t_{\text {minor }}=10.81 \mathrm{~min}$.

HRMS (ESI) $m / z$ : calcd for $\mathrm{C}_{33} \mathrm{H}_{25} \mathrm{~F}_{6} \mathrm{~N}_{2} \mathrm{O}[\mathrm{M}+\mathrm{H}]^{+}$579.1871, found: 579.1862.

(S)-4-Benzyl-4-((S,E)-1-(4-cyanophenyl)-4,4,4-trifluorobut-2-en-1-yl)-1,3-diphenyl-1H-pyrazol-5 (4H)-one (4m)

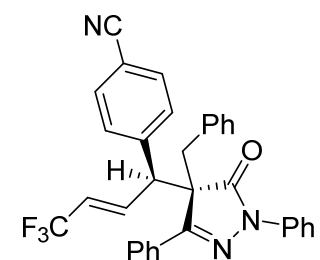

Column chromatography elution: petroleum ether/ethyl acetate $=$ $30: 1$ (v/v), colorless oil, $187.3 \mathrm{mg}, 70 \%$ yield, > 20:1 dr, $95 \%$ ee. $[\alpha]_{\mathrm{D}}^{20}=+72.50\left(\mathrm{c} 0.28, \mathrm{CH}_{2} \mathrm{Cl}_{2}\right)$.

${ }^{1} \mathrm{H}$ NMR $\left(400 \mathrm{MHz}, \mathrm{CDCl}_{3}\right) \delta 7.91(\mathrm{~d}, J=5.6 \mathrm{~Hz}, 2 \mathrm{H}), 7.66-7.53(\mathrm{~m}$, $4 \mathrm{H}), 7.46-7.42(\mathrm{~m}, 2 \mathrm{H}), 7.40-7.32(\mathrm{~m}, 4 \mathrm{H}), 7.26-7.19(\mathrm{~m}, 1 \mathrm{H}), 7.11$ $-6.97(\mathrm{~m}, 7 \mathrm{H}), 6.01(\mathrm{dq}, J=12.1,6.0 \mathrm{~Hz}, 1 \mathrm{H}), 4.34(\mathrm{~d}, J=9.9 \mathrm{~Hz}, 1 \mathrm{H}), 3.64(\mathrm{~d}, J=13.5 \mathrm{~Hz}$, $1 \mathrm{H}), 3.52(\mathrm{~d}, J=13.5 \mathrm{~Hz}, 1 \mathrm{H}) .{ }^{13} \mathrm{C} \mathrm{NMR}\left(101 \mathrm{MHz}, \mathrm{CDCl}_{3}\right.$ ) (one aromatic carbon missing) $\delta$ 173.5, 156.8, 141.9, 136.7, 135.5 (d, $J=6.6 \mathrm{~Hz}), 133.6,132.2,131.2,130.8,129.2,128.9,128.7$, 128.3, 127.6, 126.5, 126.2, 122.9 (d, $J=34.3 \mathrm{~Hz}), 122.4$ (d, $J=271.7 \mathrm{~Hz}), 120.0,118.2,112.1$, 63.2, 53.1, 41.4. ${ }^{19} \mathrm{~F}$ NMR (376 MHz, $\left.\mathrm{CDCl}_{3}\right) \delta-64.05(\mathrm{~d}, J=6.0 \mathrm{~Hz})$.

HPLC analysis: Daicel CHIRALPAK AD-H, $n$-hexane $/ i$-PrOH $=9 / 1$, flow rate $=0.8 \mathrm{~mL} / \mathrm{min}, \lambda=$ $254 \mathrm{~nm}$, retention time: $t_{\text {major }}=7.75 \mathrm{~min}, t_{\text {minor }}=9.36 \mathrm{~min}$.

HRMS (ESI) $m / z$ : calcd for $\mathrm{C}_{33} \mathrm{H}_{25} \mathrm{~F}_{3} \mathrm{~N}_{3} \mathrm{O}[\mathrm{M}+\mathrm{H}]^{+}$536.1950, found: 536.1944 .

(S)-4-Benzyl-1,3-diphenyl-4-((S,E)-4,4,4-trifluoro-1-(3,4,5-trimethoxyphenyl)but-2-en-1-yl)-1H-p yrazol-5(4H)-one (4n)

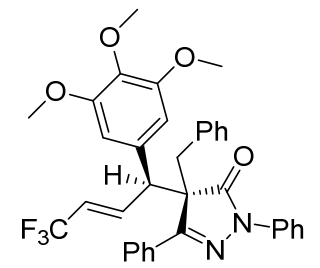

Column chromatography elution: petroleum ether/ethyl acetate $=20: 1(\mathrm{v} / \mathrm{v})$, white solid, $\mathrm{mp} 194-195{ }^{\circ} \mathrm{C}, 270.3 \mathrm{mg}, 90 \%$ yield, > 20:1 dr, 94\% ee. $[\alpha]_{\mathrm{D}}^{20}=+76.95\left(\mathrm{c} 0.56, \mathrm{CH}_{2} \mathrm{Cl}_{2}\right)$.

${ }^{1} \mathrm{H}$ NMR (400 MHz, $\left.\mathrm{CDCl}_{3}\right) \delta 7.93-7.87(\mathrm{~m}, 2 \mathrm{H}), 7.60-7.48(\mathrm{~m}, 6 \mathrm{H})$, $7.34-7.28(\mathrm{~m}, 2 \mathrm{H}), 7.20-7.12(\mathrm{~m}, 1 \mathrm{H}), 7.09-7.01(\mathrm{~m}, 5 \mathrm{H}), 5.99(\mathrm{~s}, 2 \mathrm{H})$, $5.97-5.91(\mathrm{~m}, 1 \mathrm{H}), 4.13(\mathrm{~d}, J=9.9 \mathrm{~Hz}, 1 \mathrm{H}), 3.69(\mathrm{~s}, 3 \mathrm{H}), 3.61(\mathrm{~d}, J=13.7 \mathrm{~Hz}, 1 \mathrm{H}), 3.48(\mathrm{~d}, J=$ $13.7 \mathrm{~Hz}, 1 \mathrm{H}), 3.31$ (s, 6H). ${ }^{13} \mathrm{C}$ NMR $\left(101 \mathrm{MHz}, \mathrm{CDCl}_{3}\right) \delta 174.1,157.3,152.9,137.4,137.2$, 136.5 (q, $J=6.1 \mathrm{~Hz}), 134.1,131.9,131.8,130.3,129.2,128.9,128.7,128.3,127.4,126.7,125.8$, $122.7(\mathrm{q}, J=270.7 \mathrm{~Hz}), 121.6(\mathrm{q}, J=33.8 \mathrm{~Hz}), 119.7,104.5,63.8,60.7,55.5,53.9,41.5 .{ }^{19} \mathrm{~F}$ $\operatorname{NMR}\left(377 \mathrm{MHz}, \mathrm{CDCl}_{3}\right) \delta-63.77(\mathrm{~d}, J=5.8 \mathrm{~Hz})$.

HPLC analysis: Daicel CHIRALPAK AD-H, $n$-hexane $/ i-\mathrm{PrOH}=95 / 5$, flow rate $=0.8 \mathrm{~mL} / \mathrm{min}, \lambda$ $=254 \mathrm{~nm}$, retention time: $t_{\text {major }}=9.93 \mathrm{~min}, t_{\text {minor }}=12.22 \mathrm{~min}$.

HRMS (ESI) $m / z$ : calcd for $\mathrm{C}_{35} \mathrm{H}_{32} \mathrm{~F}_{3} \mathrm{~N}_{2} \mathrm{O}_{4}[\mathrm{M}+\mathrm{H}]^{+}$601.2314, found: 601.2306.

(S)-4-Benzyl-1,3-diphenyl-4-((R,E)-4,4,4-trifluoro-1-(thiophen-2-yl)but-2-en-1-yl)-1H-pyrazol-5( $4 H)$-one (4o) 


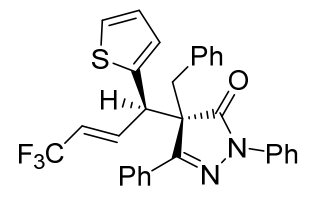

Column chromatography elution: petroleum ether/ethyl acetate $=50: 1(\mathrm{v} / \mathrm{v})$, white solid, $\mathrm{mp} 118-120{ }^{\circ} \mathrm{C}, 209.2 \mathrm{mg}, 81 \%$ yield, $>20: 1 \mathrm{dr}, 85 \%$ ee. $[\alpha]_{\mathrm{D}}^{20}=+95.65\left(\mathrm{c} 0.12, \mathrm{CH}_{2} \mathrm{Cl}_{2}\right)$.

${ }^{1} \mathrm{H}$ NMR $\left(400 \mathrm{MHz}, \mathrm{CDCl}_{3}\right) \delta 7.90(\mathrm{dd}, J=6.6,3.0 \mathrm{~Hz}, 2 \mathrm{H}), 7.59-7.46(\mathrm{~m}$, $6 \mathrm{H}), 7.36-7.30(\mathrm{~m}, 2 \mathrm{H}), 7.18(\mathrm{t}, J=7.4 \mathrm{~Hz}, 1 \mathrm{H}), 7.11-6.97(\mathrm{~m}, 6 \mathrm{H}), 6.73(\mathrm{dd}, J=5.1,3.6 \mathrm{~Hz}$, $1 \mathrm{H}), 6.59(\mathrm{~d}, J=3.1 \mathrm{~Hz}, 1 \mathrm{H}), 5.96(\mathrm{dq}, J=15.5,6.1 \mathrm{~Hz}, 1 \mathrm{H}), 4.58(\mathrm{~d}, J=9.9 \mathrm{~Hz}, 1 \mathrm{H}), 3.57(\mathrm{~d}, J$ $=13.4 \mathrm{~Hz}, 1 \mathrm{H}), 3.46(\mathrm{~d}, J=13.4 \mathrm{~Hz}, 1 \mathrm{H}) .{ }^{13} \mathrm{C} \mathrm{NMR}\left(101 \mathrm{MHz}, \mathrm{CDCl}_{3}\right) \delta$ 173.7, 157.2, 138.1, $137.1,136.5(\mathrm{q}, J=6.5 \mathrm{~Hz}), 133.9,131.4,130.5,129.2,129.0,128.7,128.2,127.4,126.7,126.6$, 125.9, 125.7, 124.9, 122.7 (q, $J=271.7 \mathrm{~Hz}), 121.6(\mathrm{q}, J=34.1 \mathrm{~Hz}), 120.2,63.8,48.5,41.6 .{ }^{19} \mathrm{~F}$ $\operatorname{NMR}\left(377 \mathrm{MHz}, \mathrm{CDCl}_{3}\right) \delta-63.91(\mathrm{~d}, J=6.1 \mathrm{~Hz})$.

HPLC analysis: Daicel CHIRALPAK AD-H, $n$-hexane $/ i$-PrOH $=50 / 1$, flow rate $=0.8 \mathrm{~mL} / \mathrm{min}, \lambda$ $=254 \mathrm{~nm}$, retention time: $t_{\text {minor }}=19.75 \mathrm{~min}, t_{\text {major }}=21.71 \mathrm{~min}$.

HRMS (ESI) $m / z$ : calcd for $\mathrm{C}_{30} \mathrm{H}_{24} \mathrm{~F}_{3} \mathrm{~N}_{2} \mathrm{OS}[\mathrm{M}+\mathrm{H}]^{+}$517.1561, found: 517.1554.

(S)-4-(3-Methylbenzyl)-1,3-diphenyl-4-((S,E)-4,4,4-trifluoro-1-( $m$-tolyl)but-2-en-1-yl)-1 $H$-pyrazo $1-5(4 H)$-one (4q)

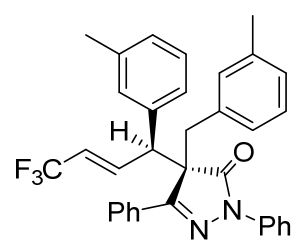

Column chromatography elution: petroleum ether/ethyl acetate $=50: 1(\mathrm{v} / \mathrm{v})$, colorless oil, $237.0 \mathrm{mg}, 88 \%$ yield, $>20: 1 \mathrm{dr}, 95 \%$ ee.

$[\alpha]_{\mathrm{D}}^{20}=+69.33\left(\mathrm{c} 0.53, \mathrm{CH}_{2} \mathrm{Cl}_{2}\right)$.

${ }^{1} \mathrm{H}$ NMR $\left(400 \mathrm{MHz}, \mathrm{CDCl}_{3}\right) \delta 7.89-7.81(\mathrm{~m}, 2 \mathrm{H}), 7.65-7.56(\mathrm{~m}, 1 \mathrm{H}), 7.55$ $-7.45(\mathrm{~m}, 5 \mathrm{H}), 7.38-7.31(\mathrm{~m}, 2 \mathrm{H}), 7.20(\mathrm{t}, J=7.4 \mathrm{~Hz}, 1 \mathrm{H}), 6.97-6.79(\mathrm{~m}$, $6 \mathrm{H}), 6.71-6.67(\mathrm{~m}, 1 \mathrm{H}), 6.65(\mathrm{~s}, 1 \mathrm{H}), 5.93(\mathrm{dq}, J=15.6,6.2 \mathrm{~Hz}, 1 \mathrm{H}), 4.19(\mathrm{~d}, J=10.0 \mathrm{~Hz}, 1 \mathrm{H})$, $3.61(\mathrm{~d}, J=13.6 \mathrm{~Hz}, 1 \mathrm{H}), 3.43(\mathrm{~d}, J=13.6 \mathrm{~Hz}, 1 \mathrm{H}), 2.06(\mathrm{~s}, 3 \mathrm{H}), 2.03(\mathrm{~s}, 3 \mathrm{H}) .{ }^{13} \mathrm{C}$ NMR $(101$ $\mathrm{MHz}, \mathrm{CDCl}_{3}$ ) (one aromatic carbon missing) $\delta 174.2,157.7,138.2,137.8,137.1,137.0$ (q, $J=6.1$ $\mathrm{Hz}), 136.3,134.1,131.9,130.3,130.0,128.8,128.7,128.6,128.5,128.3,128.1,128.0,126.8$, 126.2, 125.9, 124.9, 122.7 (q, $J=270.7 \mathrm{~Hz}), 121.4$ (q, $J=34.3 \mathrm{~Hz}), 120.3,63.6,53.7,41.5,21.1$. ${ }^{19} \mathrm{~F} \mathrm{NMR}\left(377 \mathrm{MHz}, \mathrm{CDCl}_{3}\right) \delta-63.76(\mathrm{~d}, J=6.2 \mathrm{~Hz})$.

HPLC analysis: Daicel CHIRALPAK AD-H, $n$-hexane $/ i-\mathrm{PrOH}=50 / 1$, flow rate $=0.8 \mathrm{~mL} / \mathrm{min}, \lambda$ $=254 \mathrm{~nm}$, retention time: $t_{\text {major }}=8.11 \mathrm{~min}, t_{\text {minor }}=10.20 \mathrm{~min}$.

HRMS (ESI) $m / z$ : calcd for $\mathrm{C}_{34} \mathrm{H}_{30} \mathrm{~F}_{3} \mathrm{~N}_{2} \mathrm{O}[\mathrm{M}+\mathrm{H}]^{+}$539.2310, found: 539.2301 .

(S)-4-(Naphthalen-1-ylmethyl)-1,3-diphenyl-4-((S,E)-4,4,4-trifluoro-1-( $m$-tolyl)but-2-en-1-yl)-1 Hpyrazol-5(4H)-one (4r)

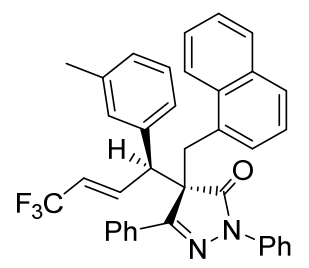

Column chromatography elution: petroleum ether/ethyl acetate $=50: 1(\mathrm{v} / \mathrm{v})$, colorless oil, $229.8 \mathrm{mg}, 80 \%$ yield, $>20: 1 \mathrm{dr}$, $96 \%$ ee.

$[\alpha]_{\mathrm{D}}^{20}=+87.55\left(\mathrm{c} 0.25, \mathrm{CH}_{2} \mathrm{Cl}_{2}\right)$.

${ }^{1} \mathrm{H}$ NMR $\left(400 \mathrm{MHz}, \mathrm{CDCl}_{3}\right) \delta 8.15(\mathrm{~d}, J=8.3 \mathrm{~Hz}, 1 \mathrm{H}), 7.79(\mathrm{~d}, J=7.4 \mathrm{~Hz}$, $2 \mathrm{H}), 7.76-7.65(\mathrm{~m}, 2 \mathrm{H}), 7.61(\mathrm{~d}, J=7.0 \mathrm{~Hz}, 1 \mathrm{H}), 7.51-7.40(\mathrm{~m}, 5 \mathrm{H}), 7.34$ $-7.23(\mathrm{~m}, 4 \mathrm{H}), 7.17-7.09(\mathrm{~m}, 3 \mathrm{H}), 6.98-6.92(\mathrm{~m}, 2 \mathrm{H}), 6.74-6.66(\mathrm{~m}, 2 \mathrm{H}), 6.09-5.97(\mathrm{~m}$, 
1H), $4.28(\mathrm{~d}, J=10.1 \mathrm{~Hz}, 1 \mathrm{H}), 4.23(\mathrm{~d}, J=15.0 \mathrm{~Hz}, 1 \mathrm{H}), 3.90(\mathrm{~d}, J=15.0 \mathrm{~Hz}, 1 \mathrm{H}), 2.02(\mathrm{~s}, 3 \mathrm{H})$. ${ }^{13} \mathrm{C}$ NMR $\left(101 \mathrm{MHz}, \mathrm{CDCl}_{3}\right) \delta 173.9,158.0,138.2,137.1(\mathrm{q}, J=6.3 \mathrm{~Hz}), 137.0,136.0,133.7$, $131.8,131.7,130.8,130.3,128.82,128.80,128.62,128.60,128.5,128.3,128.1,126.9,126.5$, 125.84, 125.80, 125.6, 125.1, 124.9, 123.7, 122.7 (q, $J=270.7 \mathrm{~Hz}), 121.7$ (q, $J=34.0 \mathrm{~Hz}), 120.1$, 62.6, 54.5, 36.2, 21.1. ${ }^{19} \mathrm{~F}$ NMR $\left(377 \mathrm{MHz}, \mathrm{CDCl}_{3}\right) \delta-63.72(\mathrm{~d}, J=5.9 \mathrm{~Hz})$.

HPLC analysis: Daicel CHIRALPAK AD-H, $n$-hexane $/ i-\mathrm{PrOH}=50 / 1$, flow rate $=0.8 \mathrm{~mL} / \mathrm{min}, \lambda$ $=254 \mathrm{~nm}$, retention time: $t_{\text {major }}=8.29 \mathrm{~min}, t_{\text {minor }}=26.40 \mathrm{~min}$.

HRMS (ESI) $m / z$ : calcd for $\mathrm{C}_{37} \mathrm{H}_{30} \mathrm{~F}_{3} \mathrm{~N}_{2} \mathrm{O}[\mathrm{M}+\mathrm{H}]^{+}$575.2310, found: 575.2304 .

(S)-4-(2-Fluorobenzyl)-1,3-diphenyl-4-((S,E)-4,4,4-trifluoro-1-( $m$-tolyl)but-2-en-1-yl)-1H-pyrazol $-5(4 H)$-one $(4 \mathbf{s})$

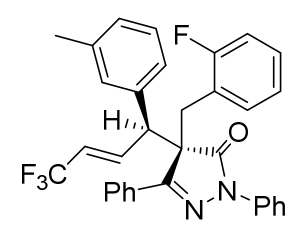

Column chromatography elution: petroleum ether/ethyl acetate $=50: 1(\mathrm{v} / \mathrm{v})$, colorless oil, $195.3 \mathrm{mg}, 72 \%$ yield, $>20: 1 \mathrm{dr}, 97 \%$ ee.

$[\alpha]_{\mathrm{D}}^{20}=+73.09\left(\mathrm{c} 0.20, \mathrm{CH}_{2} \mathrm{Cl}_{2}\right)$.

${ }^{1} \mathrm{H}$ NMR $\left(400 \mathrm{MHz}, \mathrm{CDCl}_{3}\right) \delta 7.80(\mathrm{~d}, J=7.4 \mathrm{~Hz}, 2 \mathrm{H}), 7.63-7.52(\mathrm{~m}, 3 \mathrm{H})$, $7.51-7.41(\mathrm{~m}, 3 \mathrm{H}), 7.38-7.31(\mathrm{~m}, 2 \mathrm{H}), 7.20(\mathrm{t}, J=7.4 \mathrm{~Hz}, 1 \mathrm{H}), 7.14-$ $7.01(\mathrm{~m}, 2 \mathrm{H}), 6.95-6.87(\mathrm{~m}, 2 \mathrm{H}), 6.87-6.77(\mathrm{~m}, 2 \mathrm{H}), 6.67-6.57$ (m, 2H), 5.94 (dq, $J=15.5$, $6.2 \mathrm{~Hz}, 1 \mathrm{H}), 4.17(\mathrm{~d}, J=10.1 \mathrm{~Hz}, 1 \mathrm{H}), 3.68(\mathrm{~d}, J=14.2 \mathrm{~Hz}, 1 \mathrm{H}), 3.54(\mathrm{~d}, J=14.2 \mathrm{~Hz}, 1 \mathrm{H}), 2.00$ (s, 3H). ${ }^{13} \mathrm{C} \mathrm{NMR}\left(101 \mathrm{MHz}, \mathrm{CDCl}_{3}\right) \delta 174.1,160.6$ (d, $\left.J=246.6 \mathrm{~Hz}\right), 157.8,138.1,137.1,136.7$ (q, $J=6.5 \mathrm{~Hz}), 136.0,131.2,130.7(\mathrm{~d}, J=3.7 \mathrm{~Hz}), 130.3,129.1(\mathrm{~d}, J=8.4 \mathrm{~Hz}), 128.8,128.7$, 128.55, 128.53, 128.3, $126.8(\mathrm{~d}, J=1.7 \mathrm{~Hz}), 125.9,124.9,124.0(\mathrm{~d}, J=3.7 \mathrm{~Hz}), 122.6(\mathrm{q}, J=$ $271.7 \mathrm{~Hz}), 121.6(\mathrm{q}, J=34.0 \mathrm{~Hz}), 121.5,120.1,115.4(\mathrm{~d}, J=22.9 \mathrm{~Hz}), 62.6,54.0,33.7,21.1 .{ }^{19} \mathrm{~F}$ NMR (377 MHz, $\left.\mathrm{CDCl}_{3}\right) \delta-63.83(\mathrm{~d}, J=6.2 \mathrm{~Hz}, 3 \mathrm{~F}),-114.58--114.68(\mathrm{~m}, 1 \mathrm{~F})$.

HPLC analysis: Daicel CHIRALPAK AD-H, $n$-hexane $/ i-\mathrm{PrOH}=50 / 1$, flow rate $=0.8 \mathrm{~mL} / \mathrm{min}, \lambda$ $=254 \mathrm{~nm}$, retention time: $t_{\text {major }}=8.85 \mathrm{~min}, t_{\text {minor }}=10.14 \mathrm{~min}$.

HRMS (ESI) $m / z$ : calcd for $\mathrm{C}_{33} \mathrm{H}_{27} \mathrm{~F}_{4} \mathrm{~N}_{2} \mathrm{O}[\mathrm{M}+\mathrm{H}]^{+} 543.2060$, found: 543.2051 .

(S)-4-(3,5-Bis(trifluoromethyl)benzyl)-1,3-diphenyl-4-((S,E)-4,4,4-trifluoro-1-( $m$-tolyl)but-2-en-1 -yl)-1 $H$-pyrazol-5(4H)-one (4t)

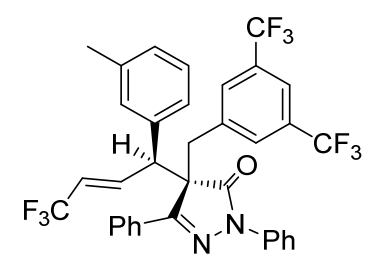

Column chromatography elution: petroleum ether/ethyl acetate $=50: 1$ (v/v), colorless oil, $287.3 \mathrm{mg}, 87 \%$ yield, $10: 1 \mathrm{dr}, 95 \%$ ee.

$[\alpha]_{\mathrm{D}}^{20}=+71.67\left(\mathrm{c} 0.60, \mathrm{CH}_{2} \mathrm{Cl}_{2}\right)$.

${ }^{1} \mathrm{H}$ NMR (400 MHz, $\left.\mathrm{CDCl}_{3}\right) \delta 7.84(\mathrm{~d}, J=6.1 \mathrm{~Hz}, 2 \mathrm{H}), 7.62-7.50(\mathrm{~m}$, $5 \mathrm{H}), 7.44-7.35(\mathrm{~m}, 4 \mathrm{H}), 7.36-7.29(\mathrm{~m}, 2 \mathrm{H}), 7.20(\mathrm{t}, J=7.4 \mathrm{~Hz}, 1 \mathrm{H})$, $6.98-6.90(\mathrm{~m}, 2 \mathrm{H}), 6.76-6.68(\mathrm{~m}, 2 \mathrm{H}), 5.98(\mathrm{dq}, J=14.7,6.3 \mathrm{~Hz}, 1 \mathrm{H}), 4.30(\mathrm{~d}, J=9.9 \mathrm{~Hz}, 1 \mathrm{H})$, $3.71(\mathrm{~d}, J=13.5 \mathrm{~Hz}, 1 \mathrm{H}), 3.52(\mathrm{~d}, J=13.5 \mathrm{~Hz}, 1 \mathrm{H}), 2.02(\mathrm{~s}, 3 \mathrm{H}) .{ }^{13} \mathrm{C} \mathrm{NMR}\left(101 \mathrm{MHz}, \mathrm{CDCl}_{3}\right) \delta$ 173.4, 156.6, 138.4, 136.9, 136.6, 136.3 (q, $J=6.3 \mathrm{~Hz}), 135.7,131.5$ (q, $J=33.3 \mathrm{~Hz}), 131.1$, $130.9,129.7,129.1,129.0,128.7,128.5,128.3,126.3,126.2,124.8,122.6$ (q, $J=270.7 \mathrm{~Hz})$, $122.9(\mathrm{q}, J=273.7 \mathrm{~Hz}), 121.9(\mathrm{q}, J=34.0 \mathrm{~Hz}), 121.2(\mathrm{q}, J=9.1 \mathrm{~Hz}), 120.1,63.4,53.6,40.5,21.1$. ${ }^{19} \mathrm{~F}$ NMR $\left(377 \mathrm{MHz}, \mathrm{CDCl}_{3}\right) \delta-63.20(\mathrm{~s}, 6 \mathrm{~F}),-63.91(\mathrm{~d}, J=6.2 \mathrm{~Hz}, 3 \mathrm{~F})$. 
HPLC analysis: Daicel CHIRALPAK AD-H, $n$-hexane $/ i-\mathrm{PrOH}=99 / 1$, flow rate $=0.8 \mathrm{~mL} / \mathrm{min}, \lambda$ $=254 \mathrm{~nm}$, retention time: $t_{\text {major }}=7.25 \mathrm{~min}, t_{\text {minor }}=7.58 \mathrm{~min}$.

HRMS (ESI) $m / z$ : calcd for $\mathrm{C}_{35} \mathrm{H}_{26} \mathrm{~F}_{9} \mathrm{~N}_{2} \mathrm{O}[\mathrm{M}+\mathrm{H}]^{+}$661.1901, found: 661.1901 .

(S)-4-(4-Nitrobenzyl)-1,3-diphenyl-4-((S,E)-4,4,4-trifluoro-1-( $m$-tolyl)but-2-en-1-yl)-1H-pyrazol$5(4 H)$-one $(4 \mathbf{u})$<smiles>Cc1cccc(C(=CC(F)(F)F)C(Cc2cccc([N+](=O)[O-])c2)C2(Cc3ccccc3)NN(c3ccccc3)C2c2ccccc2)c1</smiles>

Column chromatography elution: petroleum ether/ethyl acetate $=50: 1$ (v/v), colorless oil, $196.5 \mathrm{mg}, 69 \%$ yield, $>20: 1 \mathrm{dr}, 77 \%$ ee.

$[\alpha]_{\mathrm{D}}{ }^{20}=+46.93\left(\mathrm{c} 0.41, \mathrm{CH}_{2} \mathrm{Cl}_{2}\right)$.

${ }^{1} \mathrm{H}$ NMR $\left(400 \mathrm{MHz}, \mathrm{CDCl}_{3}\right) \delta 7.91(\mathrm{~d}, J=8.6 \mathrm{~Hz}, 2 \mathrm{H}), 7.89-7.80(\mathrm{~m}$, $2 \mathrm{H}), 7.62-7.50(\mathrm{~m}, 4 \mathrm{H}), 7.47(\mathrm{~d}, J=8.3 \mathrm{~Hz}, 2 \mathrm{H}), 7.36-7.31(\mathrm{~m}, 2 \mathrm{H})$, $7.25-7.15(\mathrm{~m}, 3 \mathrm{H}), 6.97-6.89(\mathrm{~m}, 2 \mathrm{H}), 6.72-6.58(\mathrm{~m}, 2 \mathrm{H}), 6.05-5.92(\mathrm{~m}, 1 \mathrm{H}), 4.24(\mathrm{~d}, J=$ $10.0 \mathrm{~Hz}, 1 \mathrm{H}), 3.69(\mathrm{~d}, J=13.5 \mathrm{~Hz}, 1 \mathrm{H}), 3.57(\mathrm{~d}, J=13.5 \mathrm{~Hz}, 1 \mathrm{H}), 2.01(\mathrm{~s}, 3 \mathrm{H}) .{ }^{13} \mathrm{C}$ NMR $(101$ $\left.\mathrm{MHz}, \mathrm{CDCl}_{3}\right) \delta 173.5,156.8,147.2,142.0,138.3,136.7,136.4(\mathrm{q}, J=6.7 \mathrm{~Hz}), 135.7,131.3$, $130.8,130.3,129.1,129.0,128.8,128.4,128.3,126.5,126.2,124.8,123.4,122.6$ (q, $J=270.7$ $\mathrm{Hz}), 121.9(\mathrm{q}, J=33.9 \mathrm{~Hz}), 119.9,63.3,53.7,40.7,21.1 .{ }^{19} \mathrm{~F}$ NMR $\left(377 \mathrm{MHz}, \mathrm{CDCl}_{3}\right) \delta-63.82(\mathrm{~d}$, $J=5.9 \mathrm{~Hz})$.

HPLC analysis: Daicel CHIRALPAK AD-H, $n$-hexane $/ i-\mathrm{PrOH}=95 / 5$, flow rate $=0.8 \mathrm{~mL} / \mathrm{min}, \lambda$ $=254 \mathrm{~nm}$, retention time: $t_{\text {major }}=13.37 \mathrm{~min}, t_{\text {minor }}=16.54 \mathrm{~min}$.

HRMS (ESI) $m / z$ : calcd for $\mathrm{C}_{33} \mathrm{H}_{27} \mathrm{~F}_{3} \mathrm{~N}_{3} \mathrm{O}_{3}[\mathrm{M}+\mathrm{H}]^{+} 570.2005$, found: 570.1993 .

(S)-4-Benzyl-1-phenyl-3-(o-tolyl)-4-((S,E)-4,4,4-trifluoro-1-( $m$-tolyl)but-2-en-1-yl)-1H-pyrazol-5( $4 H)$-one $(\mathbf{4 v})$

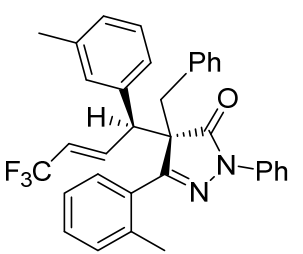

Column chromatography elution: petroleum ether/ethyl acetate $=50: 1(\mathrm{v} / \mathrm{v})$, colorless oil, $239.7 \mathrm{mg}, 89 \%$ yield, $>20: 1 \mathrm{dr}, 94 \%$ ee. $[\alpha]_{\mathrm{D}}^{20}=+97.13\left(\mathrm{c} 0.21, \mathrm{CH}_{2} \mathrm{Cl}_{2}\right)$.

${ }^{1} \mathrm{H}$ NMR $\left(400 \mathrm{MHz}, \mathrm{CDCl}_{3}\right) \delta 7.70(\mathrm{~d}, J=7.8 \mathrm{~Hz}, 1 \mathrm{H}), 7.59(\mathrm{~s}, 1 \mathrm{H}), 7.58-$ $7.51(\mathrm{~m}, 1 \mathrm{H}), 7.48-7.39(\mathrm{~m}, 3 \mathrm{H}), 7.36-7.29(\mathrm{~m}, 3 \mathrm{H}), 7.18(\mathrm{t}, J=7.4 \mathrm{~Hz}$, 1H), $7.11-6.99(\mathrm{~m}, 5 \mathrm{H}), 6.97-6.88(\mathrm{~m}, 2 \mathrm{H}), 6.67(\mathrm{~d}, J=6.7 \mathrm{~Hz}, 1 \mathrm{H}), 6.63(\mathrm{~s}, 1 \mathrm{H}), 5.92(\mathrm{dq}, J=$ 15.6, $6.2 \mathrm{~Hz}, 1 \mathrm{H}), 4.16(\mathrm{~d}, J=10.0 \mathrm{~Hz}, 1 \mathrm{H}), 3.59(\mathrm{~d}, J=13.6 \mathrm{~Hz}, 1 \mathrm{H}), 3.47$ (d, $J=13.6 \mathrm{~Hz}, 1 \mathrm{H})$, $2.43(\mathrm{~s}, 3 \mathrm{H}), 2.03(\mathrm{~s}, 3 \mathrm{H}) .{ }^{13} \mathrm{C} \mathrm{NMR}\left(101 \mathrm{MHz}, \mathrm{CDCl}_{3}\right) \delta 174.0,157.7,138.7,138.1,137.04$, $137.00(\mathrm{q}, J=6.7 \mathrm{~Hz}), 136.3,134.3,131.7,131.2,129.3,128.7,128.67,128.60,128.5,128.3$, 128.2, 127.4, 127.3, 125.9, 125.0, 123.9, 123.0 (q, $J=208.1 \mathrm{~Hz}), 121.5$ (q, $J=33.9 \mathrm{~Hz}), 120.4$, 63.6, 53.6, 41.4, 21.6, 21.1. ${ }^{19} \mathrm{~F}$ NMR $\left(377 \mathrm{MHz}, \mathrm{CDCl}_{3}\right) \delta-63.78(\mathrm{~d}, J=6.1 \mathrm{~Hz})$.

HPLC analysis: Daicel CHIRALPAK AD-H, $n$-hexane $/ i-\mathrm{PrOH}=50 / 1$, flow rate $=0.8 \mathrm{~mL} / \mathrm{min}, \lambda$ $=254 \mathrm{~nm}$, retention time: $t_{\text {major }}=7.62 \mathrm{~min}, t_{\text {minor }}=10.52 \mathrm{~min}$.

HRMS (ESI) $m / z$ : calcd for $\mathrm{C}_{34} \mathrm{H}_{30} \mathrm{~F}_{3} \mathrm{~N}_{2} \mathrm{O}[\mathrm{M}+\mathrm{H}]^{+}$539.2310, found: 539.2311 .

(S)-4-Benzyl-1-phenyl-3-( $m$-tolyl)-4-((S,E)-4,4,4-trifluoro-1-( $m$-tolyl)but-2-en-1-yl)-1H-pyrazol-5 $(4 H)$-one $(4 \mathbf{w})$ 


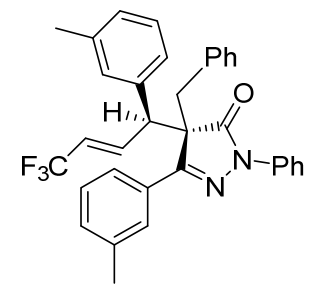

Column chromatography elution: petroleum ether/ethyl acetate $=50: 1(\mathrm{v} / \mathrm{v})$, colorless oil, $237.0 \mathrm{mg}, 88 \%$ yield, $>20: 1 \mathrm{dr}, 92 \%$ ee.

$[\alpha]_{\mathrm{D}}^{20}=+72.00\left(\mathrm{c} 0.43, \mathrm{CH}_{2} \mathrm{Cl}_{2}\right)$.

${ }^{1} \mathrm{H}$ NMR $\left(400 \mathrm{MHz}, \mathrm{CDCl}_{3}\right) \delta 7.70(\mathrm{~d}, J=7.6 \mathrm{~Hz}, 1 \mathrm{H}), 7.64-7.52(\mathrm{~m}, 2 \mathrm{H})$, $7.47-7.38(\mathrm{~m}, 3 \mathrm{H}), 7.36-7.28(\mathrm{~m}, 3 \mathrm{H}), 7.18(\mathrm{t}, J=7.4 \mathrm{~Hz}, 1 \mathrm{H}), 7.11-$ $6.99(\mathrm{~m}, 5 \mathrm{H}), 6.99-6.88(\mathrm{~m}, 2 \mathrm{H}), 6.74-6.60(\mathrm{~m}, 2 \mathrm{H}), 6.01-5.86(\mathrm{~m}, 1 \mathrm{H}), 4.17(\mathrm{~d}, J=10.0 \mathrm{~Hz}$, $1 \mathrm{H}), 3.60(\mathrm{~d}, J=13.5 \mathrm{~Hz}, 1 \mathrm{H}), 3.48(\mathrm{~d}, J=13.5 \mathrm{~Hz}, 1 \mathrm{H}), 2.43(\mathrm{~s}, 3 \mathrm{H}), 2.03(\mathrm{~s}, 3 \mathrm{H}) .{ }^{13} \mathrm{C} \mathrm{NMR}$ $\left(101 \mathrm{MHz}, \mathrm{CDCl}_{3}\right) \delta 174.1,157.8,138.7,138.1,137.1,137.0$ (q, $\left.J=6.7 \mathrm{~Hz}\right), 136.4,134.3,131.8$, $131.3,129.3,128.8,128.7,128.66,128.65,128.3,128.2,127.5,127.3,125.9,125.0,123.9,122.8$ $(\mathrm{q}, J=270.7 \mathrm{~Hz}), 121.5$ (q, $J=34.3 \mathrm{~Hz}), 120.4,63.7,53.6,41.4,21.6,21.2 .{ }^{19} \mathrm{~F}$ NMR $(377 \mathrm{MHz}$, $\left.\mathrm{CDCl}_{3}\right) \delta-63.77(\mathrm{~d}, J=5.9 \mathrm{~Hz})$.

HPLC analysis: Daicel CHIRALPAK AD-H, $n$-hexane $/ i-\mathrm{PrOH}=50 / 1$, flow rate $=0.8 \mathrm{~mL} / \mathrm{min}, \lambda$ $=254 \mathrm{~nm}$, retention time: $t_{\text {major }}=7.28 \mathrm{~min}, t_{\text {minor }}=10.17 \mathrm{~min}$.

HRMS (ESI) $m / z$ : calcd for $\mathrm{C}_{34} \mathrm{H}_{30} \mathrm{~F}_{3} \mathrm{~N}_{2} \mathrm{O}[\mathrm{M}+\mathrm{H}]^{+}$539.2310, found: 539.2310 .

(S)-4-Benzyl-1-phenyl-3-(p-tolyl)-4-((S,E)-4,4,4-trifluoro-1-( $m$-tolyl)but-2-en-1-yl)-1H-pyrazol-5( $4 H)$-one $(\mathbf{4} \mathbf{x})$

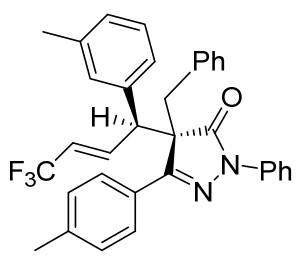

Column chromatography elution: petroleum ether/ethyl acetate $=50: 1(\mathrm{v} / \mathrm{v})$, white solid, mp $61-62{ }^{\circ} \mathrm{C}, 223.5 \mathrm{mg}, 83 \%$ yield, $>20: 1 \mathrm{dr}, 94 \%$ ee.

$[\alpha]_{\mathrm{D}}^{20}=+77.90\left(\mathrm{c} 0.44, \mathrm{CH}_{2} \mathrm{Cl}_{2}\right)$.

${ }^{1} \mathrm{H}$ NMR $\left(400 \mathrm{MHz}, \mathrm{CDCl}_{3}\right) \delta 7.76(\mathrm{~d}, J=8.6 \mathrm{~Hz}, 2 \mathrm{H}), 7.66-7.54(\mathrm{~m}, 1 \mathrm{H})$, $7.50-7.41(\mathrm{~m}, 2 \mathrm{H}), 7.37-7.28(\mathrm{~m}, 4 \mathrm{H}), 7.17(\mathrm{t}, J=7.4 \mathrm{~Hz}, 1 \mathrm{H}), 7.11-$ $7.01(\mathrm{~m}, 5 \mathrm{H}), 6.97-6.88(\mathrm{~m}, 2 \mathrm{H}), 6.66(\mathrm{~d}, J=6.5 \mathrm{~Hz}, 2 \mathrm{H}), 5.93(\mathrm{dq}, J=12.4,6.0 \mathrm{~Hz}, 1 \mathrm{H}), 4.18$ (d, $J=10.0 \mathrm{~Hz}, 1 \mathrm{H}), 3.60$ (d, $J=13.6 \mathrm{~Hz}, 1 \mathrm{H}), 3.46$ (d, $J=13.6 \mathrm{~Hz}, 1 \mathrm{H}), 2.48$ (s, 3H), 2.03 (s, $3 \mathrm{H}) .{ }^{13} \mathrm{C}$ NMR $\left(101 \mathrm{MHz}, \mathrm{CDCl}_{3}\right) \delta 174.0,157.6,140.7,138.1,137.2,137.1$ (q, $J=6.7 \mathrm{~Hz}$ ), 136.3, 134.4, 129.6, 129.3, 129.1, 128.7, 128.6, 128.5, 128.3, 128.2, 127.3, 126.7, 125.8, 125.0, $122.8(\mathrm{q}, J=271.7 \mathrm{~Hz}), 121.4(\mathrm{q}, J=33.3 \mathrm{~Hz}), 120.3,63.6,53.7,41.3,21.6,21.2 .{ }^{19} \mathrm{~F}$ NMR $(377$ $\left.\mathrm{MHz}, \mathrm{CDCl}_{3}\right) \delta-63.75(\mathrm{~d}, J=6.0 \mathrm{~Hz})$.

HPLC analysis: Daicel CHIRALPAK AD-H, $n$-hexane $/ i-\mathrm{PrOH}=50 / 1$, flow rate $=0.8 \mathrm{~mL} / \mathrm{min}, \lambda$ $=254 \mathrm{~nm}$, retention time: $t_{\text {major }}=8.74 \mathrm{~min}, t_{\text {minor }}=10.30 \mathrm{~min}$.

HRMS (ESI) $m / z$ : calcd for $\mathrm{C}_{34} \mathrm{H}_{30} \mathrm{~F}_{3} \mathrm{~N}_{2} \mathrm{O}[\mathrm{M}+\mathrm{H}]^{+}$539.2310, found: 539.2298 .

(S)-4-Benzyl-3-(naphthalen-2-yl)-1-phenyl-4-(( $S, E)-4,4,4-$ trifluoro-1-( $m$-tolyl)but-2-en-1-yl)-1H-p yrazol-5(4H)-one (4y)

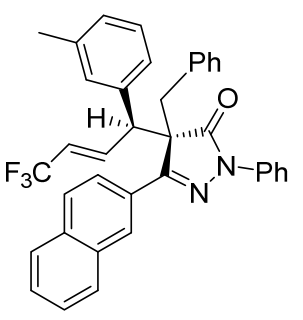

Column chromatography elution: petroleum ether/ethyl acetate $=50: 1(\mathrm{v} / \mathrm{v})$, white solid, $\mathrm{mp} 88-89{ }^{\circ} \mathrm{C}, 232.7 \mathrm{mg}, 81 \%$ yield, $>20: 1 \mathrm{dr}, 96 \%$ ee. $[\alpha]_{\mathrm{D}}^{20}=+72.74\left(\mathrm{c} 0.58, \mathrm{CH}_{2} \mathrm{Cl}_{2}\right)$.

${ }^{1} \mathrm{H}$ NMR $\left(400 \mathrm{MHz}, \mathrm{CDCl}_{3}\right) \delta 8.32(\mathrm{~s}, 1 \mathrm{H}), 8.04-7.97(\mathrm{~m}, 1 \mathrm{H}), 7.96-$ $7.87(\mathrm{~m}, 3 \mathrm{H}), 7.66-7.54(\mathrm{~m}, 3 \mathrm{H}), 7.49$ (d, $J=7.9 \mathrm{~Hz}, 2 \mathrm{H}), 7.38-7.33(\mathrm{~m}$, 
2H), $7.24-7.17(\mathrm{~m}, 1 \mathrm{H}), 7.07-6.96(\mathrm{~m}, 5 \mathrm{H}), 6.93-6.84(\mathrm{~m}, 2 \mathrm{H}), 6.71-6.60(\mathrm{~m}, 2 \mathrm{H}), 5.98(\mathrm{dq}$, $J=12.4,6.0 \mathrm{~Hz}, 1 \mathrm{H}), 4.30(\mathrm{~d}, J=9.9 \mathrm{~Hz}, 1 \mathrm{H}), 3.69(\mathrm{~d}, J=13.5 \mathrm{~Hz}, 1 \mathrm{H}), 3.61(\mathrm{~d}, J=13.5 \mathrm{~Hz}$, $1 \mathrm{H}), 1.96(\mathrm{~s}, 3 \mathrm{H}) .{ }^{13} \mathrm{C} \mathrm{NMR}\left(101 \mathrm{MHz}, \mathrm{CDCl}_{3}\right.$ ) (two aromatic carbons missing) $\delta 174.1,157.4$, $138.2,137.1,137.0$ (q, $J=6.5 \mathrm{~Hz}), 136.3,134.3,134.1,132.9,129.5,129.3,128.8,128.7,128.5$, $128.4,128.3,128.0,127.6,127.3,127.0,126.6,126.0,125.1,123.9,122.8$ (q, $J=270.7 \mathrm{~Hz})$, $121.6(\mathrm{q}, J=33.9 \mathrm{~Hz}), 120.4,63.8,53.8,41.5,21.1 .{ }^{19} \mathrm{~F} \mathrm{NMR}\left(377 \mathrm{MHz}, \mathrm{CDCl}_{3}\right) \delta-63.74(\mathrm{~d}, J=$ $5.9 \mathrm{~Hz})$.

HPLC analysis: Daicel CHIRALPAK AD-H, $n$-hexane $/ i-\mathrm{PrOH}=50 / 1$, flow rate $=0.8 \mathrm{~mL} / \mathrm{min}, \lambda$ $=254 \mathrm{~nm}$, retention time: $t_{\text {major }}=10.16 \mathrm{~min}, t_{\text {minor }}=16.43 \mathrm{~min}$.

HRMS (ESI) $m / z$ : calcd for $\mathrm{C}_{37} \mathrm{H}_{30} \mathrm{~F}_{3} \mathrm{~N}_{2} \mathrm{O}[\mathrm{M}+\mathrm{H}]^{+}$575.2310, found: 575.2299 .

(S)-4-Benzyl-1-phenyl-3-(thiophen-2-yl)-4-((S,E)-4,4,4-trifluoro-1-( $m$-tolyl)but-2-en-1-yl)-1 $H$-pyr azol-5(4H)-one $(\mathbf{4 z})$

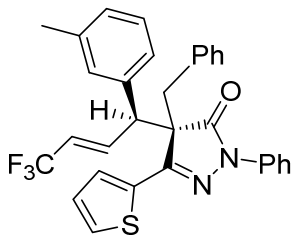

Column chromatography elution: petroleum ether/ethyl acetate $=50: 1(\mathrm{v} / \mathrm{v})$, white solid, mp $48-49^{\circ} \mathrm{C}, 222.9 \mathrm{mg}, 84 \%$ yield, $10: 1 \mathrm{dr}, 97 \%$ ee.

$[\alpha]_{\mathrm{D}}^{20}=+59.42\left(\mathrm{c} 0.31, \mathrm{CH}_{2} \mathrm{Cl}_{2}\right)$.

${ }^{1} \mathrm{H}$ NMR $\left(400 \mathrm{MHz}, \mathrm{CDCl}_{3}\right) \delta 7.64(\mathrm{~d}, J=3.4 \mathrm{~Hz}, 1 \mathrm{H}), 7.62-7.53(\mathrm{~m}, 1 \mathrm{H})$, $7.49(\mathrm{~d}, J=5.1 \mathrm{~Hz}, 1 \mathrm{H}), 7.42-7.35(\mathrm{~m}, 2 \mathrm{H}), 7.33-7.27(\mathrm{~m}, 2 \mathrm{H}), 7.25-$ $7.21(\mathrm{~m}, 1 \mathrm{H}), 7.16(\mathrm{t}, J=7.4 \mathrm{~Hz}, 1 \mathrm{H}), 7.12-7.00(\mathrm{~m}, 5 \mathrm{H}), 7.00-6.90(\mathrm{~m}, 2 \mathrm{H}), 6.74(\mathrm{~d}, J=6.0$ $\mathrm{Hz}, 2 \mathrm{H}), 5.93$ (dq, $J=12.3,6.1 \mathrm{~Hz}, 1 \mathrm{H}), 4.14(\mathrm{~d}, J=9.9 \mathrm{~Hz}, 1 \mathrm{H}), 3.58$ (d, $J=13.5 \mathrm{~Hz}, 1 \mathrm{H}), 3.34$ $(\mathrm{d}, J=13.5 \mathrm{~Hz}, 1 \mathrm{H}), 2.06(\mathrm{~s}, 3 \mathrm{H}) \cdot{ }^{13} \mathrm{C} \mathrm{NMR}\left(101 \mathrm{MHz}, \mathrm{CDCl}_{3}\right.$ ) (one aromatic carbon missing) $\delta$ 173.4, 154.0, 138.3, 136.9, $136.8(\mathrm{q}, J=6.5 \mathrm{~Hz}), 136.2,135.4,134.1,129.4,128.8,128.6,128.43$, $128.41,128.3,127.6,127.3,127.2,125.9,124.9,122.6(\mathrm{q}, J=271.7 \mathrm{~Hz}), 121.6(\mathrm{q}, J=34.0 \mathrm{~Hz})$, 120.4, 63.7, 54.1, 41.1, 21.2. ${ }^{19} \mathrm{~F}$ NMR $\left(377 \mathrm{MHz}, \mathrm{CDCl}_{3}\right) \delta-63.79(\mathrm{~d}, J=6.1 \mathrm{~Hz})$.

HPLC analysis: Daicel CHIRALPAK AD-H, $n$-hexane $/ i$-PrOH $=50 / 1$, flow rate $=0.8 \mathrm{~mL} / \mathrm{min}, \lambda$ $=254 \mathrm{~nm}$, retention time: $t_{\text {major }}=14.32 \mathrm{~min}, t_{\text {minor }}=16.49 \mathrm{~min}$.

HRMS (ESI) $m / z$ : calcd for $\mathrm{C}_{31} \mathrm{H}_{26} \mathrm{~F}_{3} \mathrm{~N}_{2} \mathrm{OS}[\mathrm{M}+\mathrm{H}]^{+} 531.1718$, found: 531.1711 .

(S)-4-Benzyl-3-methyl-1-phenyl-4-((S,E)-4,4,4-trifluoro-1-(m-tolyl)but-2-en-1-yl)-1H-pyrazol-5(4 $H)$-one (4aa)

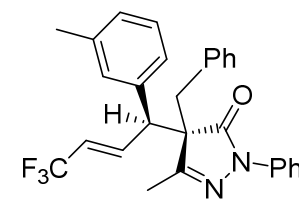

Column chromatography elution: petroleum ether/ethyl acetate $=50: 1(\mathrm{v} / \mathrm{v})$, colorless oil, $208.1 \mathrm{mg}, 90 \%$ yield, $3: 1 \mathrm{dr}, 92 \%$ ee.

$[\alpha]_{\mathrm{D}}^{20}=+32.47\left(\mathrm{c} 0.65, \mathrm{CH}_{2} \mathrm{Cl}_{2}\right)$.

$7.20-7.04(\mathrm{~m}, 7 \mathrm{H}), 7.02-6.90(\mathrm{~m}, 3 \mathrm{H}), 5.79(\mathrm{dq}, J=12.3,6.0 \mathrm{~Hz}, 1 \mathrm{H}), 3.65(\mathrm{~d}, J=9.9 \mathrm{~Hz}, 1 \mathrm{H})$, $3.44(\mathrm{~d}, J=13.5 \mathrm{~Hz}, 1 \mathrm{H}), 2.81(\mathrm{~d}, J=13.5 \mathrm{~Hz}, 1 \mathrm{H}), 2.14(\mathrm{~s}, 3 \mathrm{H}), 2.03(\mathrm{~s}, 3 \mathrm{H}) .{ }^{13} \mathrm{C}$ NMR $(101$ $\left.\mathrm{MHz}, \mathrm{CDCl}_{3}\right) \delta 173.7,159.9,138.8,137.1,136.9(\mathrm{q}, J=6.6 \mathrm{~Hz}), 136.6,134.3,129.1,129.0$, 128.9, 128.7, 128.5, 128.1, 127.5, 125.6, 124.8, 122.6 (q, $J=270.7 \mathrm{~Hz}), 121.3$ (q, $J=33.3 \mathrm{~Hz})$, 120.0, 63.4, 53.6, 40.6, 21.3, 14.9. ${ }^{19} \mathrm{~F}$ NMR $\left(377 \mathrm{MHz}, \mathrm{CDCl}_{3}\right) \delta-63.85(\mathrm{~d}, J=6.0 \mathrm{~Hz})$. 
HPLC analysis: Daicel CHIRALPAK AD-H, $n$-hexane $/ i-\mathrm{PrOH}=50 / 1$, flow rate $=0.8 \mathrm{~mL} / \mathrm{min}, \lambda$ $=254 \mathrm{~nm}$, retention time: $t_{\text {major }}=12.39 \mathrm{~min}, t_{\text {minor }}=16.49 \mathrm{~min}$.

HRMS (ESI) $m / z$ : calcd for $\mathrm{C}_{28} \mathrm{H}_{26} \mathrm{~F}_{3} \mathrm{~N}_{2} \mathrm{O}[\mathrm{M}+\mathrm{H}]^{+}$463.1997, found: 463.1986 .

(S)-4-(4-Bromobenzyl)-1,3-diphenyl-4-((S,E)-4,4,4-trifluoro-1-(3,4,5-trimethoxyphenyl)but-2-en1-yl)-1 $H$-pyrazol-5(4H)-one (4ab)

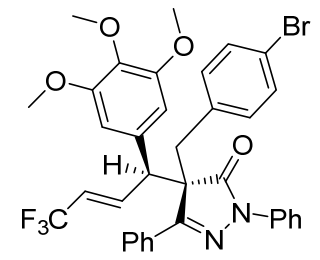

Column chromatography elution: petroleum ether/ethyl acetate $=20: 1(\mathrm{v} / \mathrm{v})$, white solid, mp $194-195{ }^{\circ} \mathrm{C}, 302.4 \mathrm{mg}, 89 \%$ yield, $>20: 1 \mathrm{dr}, 98 \%$ ee. $[\alpha]_{\mathrm{D}}^{20}=+64.80\left(\mathrm{c} 0.25, \mathrm{CH}_{2} \mathrm{Cl}_{2}\right)$.

${ }^{1} \mathrm{H}$ NMR $\left(400 \mathrm{MHz}, \mathrm{CDCl}_{3}\right) \delta 7.89(\mathrm{~d}, J=1.8 \mathrm{~Hz}, 2 \mathrm{H}), 7.61-7.43(\mathrm{~m}, 6 \mathrm{H})$, $7.38-7.29(\mathrm{~m}, 2 \mathrm{H}), 7.22-7.14(\mathrm{~m}, 3 \mathrm{H}), 6.89$ (d, $J=7.6 \mathrm{~Hz}, 2 \mathrm{H}), 5.98(\mathrm{~s}$, 2H), $5.97-5.90(\mathrm{~m}, 1 \mathrm{H}), 4.12(\mathrm{~d}, J=9.8 \mathrm{~Hz}, 1 \mathrm{H}), 3.69(\mathrm{~s}, 3 \mathrm{H}), 3.55(\mathrm{~d}, J=13.5 \mathrm{~Hz}, 1 \mathrm{H}), 3.43(\mathrm{q}$, $J=13.5 \mathrm{~Hz}, 1 \mathrm{H}), 3.30$ (s, 6H). ${ }^{13} \mathrm{C}$ NMR $\left(101 \mathrm{MHz}, \mathrm{CDCl}_{3}\right) \delta 173.9,157.0,152.9,137.5,137.0$, 136.3 (q, $J=6.4 \mathrm{~Hz}$ ), 133.1, 131.7, 131.5, 131.4, 130.9, 130.5, 129.0, 128.8, 126.6, 126.0, 122.6 $(\mathrm{q}, J=270.7 \mathrm{~Hz}), 121.8(\mathrm{q}, J=34.0 \mathrm{~Hz}), 121.5,119.6,104.5,63.6,60.7,55.5,53.9,40.7 .{ }^{19} \mathrm{~F}$ $\operatorname{NMR}\left(377 \mathrm{MHz}, \mathrm{CDCl}_{3}\right) \delta-63.79(\mathrm{~d}, J=5.9 \mathrm{~Hz})$.

HPLC analysis: Daicel CHIRALPAK AD-H, $n$-hexane $/ i-\mathrm{PrOH}=95 / 5$, flow rate $=0.8 \mathrm{~mL} / \mathrm{min}, \lambda$ $=254 \mathrm{~nm}$, retention time: $t_{\text {major }}=19.54 \mathrm{~min}, t_{\text {minor }}=26.65 \mathrm{~min}$.

HRMS (ESI) $m / z$ : calcd for $\mathrm{C}_{35} \mathrm{H}_{31} \mathrm{BrF}_{3} \mathrm{~N}_{2} \mathrm{O}_{4}[\mathrm{M}+\mathrm{H}]^{+}$679.1419, found: 679.1416.

4-Benzyl-1,3-diphenyl-4-(4,4,4-trifluoro-1-phenylbut-1-en-1-yl)-1 $H$-pyrazol-5(4H)-one (5a)

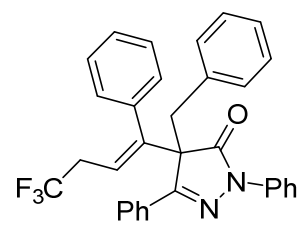

Column chromatography elution: petroleum ether/ethyl acetate $=50: 1(\mathrm{v} / \mathrm{v})$, colorless oil.

${ }^{1} \mathrm{H}$ NMR (400 MHz, $\left.\mathrm{CDCl}_{3}\right) \delta 7.89(\mathrm{~d}, J=8.4 \mathrm{~Hz}, 2 \mathrm{H}), 7.48-7.40(\mathrm{~m}, 3 \mathrm{H})$, $7.30(\mathrm{~d}, J=8.4 \mathrm{~Hz}, 2 \mathrm{H}), 7.22(\mathrm{t}, J=7.9 \mathrm{~Hz}, 2 \mathrm{H}), 7.16-7.05(\mathrm{~m}, 4 \mathrm{H}), 7.02-$ $6.99(\mathrm{~m}, 1 \mathrm{H}), 6.98-6.91(\mathrm{~m}, 4 \mathrm{H}), 6.79(\mathrm{~d}, J=7.8 \mathrm{~Hz}, 2 \mathrm{H}), 6.18(\mathrm{t}, J=15.6$ $\mathrm{Hz}, 1 \mathrm{H}), 3.63(\mathrm{~d}, J=12.6 \mathrm{~Hz}, 1 \mathrm{H}), 3.54(\mathrm{~d}, J=12.6 \mathrm{~Hz}, 1 \mathrm{H}), 2.85-2.62(\mathrm{~m}, 2 \mathrm{H}) .{ }^{13} \mathrm{C}$ NMR $(101$ $\left.\mathrm{MHz}, \mathrm{CDCl}_{3}\right) \delta 173.3,156.9,144.1,137.1,135.5,133.7,131.8,130.5,129.7,128.9,128.8,128.6$, $128.5(\mathrm{q}, J=259.7 \mathrm{~Hz}), 128.4,128.3,128.0,127.3,126.4,125.9,120.4,119.7$ (q, $J=3.5 \mathrm{~Hz})$, 66.0, 39.2, $34.4(\mathrm{q}, J=29.7 \mathrm{~Hz}) .{ }^{19} \mathrm{~F}$ NMR $\left(377 \mathrm{MHz}, \mathrm{CDCl}_{3}\right) \delta-65.23(\mathrm{td}, J=10.6,0.9 \mathrm{~Hz})$. HRMS (ESI) $m / z$ : calcd for $\mathrm{C}_{32} \mathrm{H}_{26} \mathrm{~F}_{3} \mathrm{~N}_{2} \mathrm{O}[\mathrm{M}+\mathrm{H}]^{+}$511.1997, found: 511.1987.

(S)-4-Benzyl-1,3-diphenyl-4-((S)-4,4,4-trifluoro-1-phenylbutyl)-1H-pyrazol-5(4H)-one (6a)

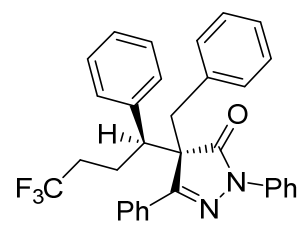

Column chromatography elution: petroleum ether/ethyl acetate $=50: 1(\mathrm{v} / \mathrm{v})$, colorless oil, $222.7 \mathrm{mg}, 87 \%$ yield, $>20: 1 \mathrm{dr}, 95 \%$ ee.

$[\alpha]_{\mathrm{D}}^{20}=+19.00\left(\mathrm{c} 0.30, \mathrm{CH}_{2} \mathrm{Cl}_{2}\right)$.

${ }^{1} \mathrm{H} \mathrm{NMR}\left(400 \mathrm{MHz}, \mathrm{CDCl}_{3}\right) \delta 7.82(\mathrm{~d}, J=7.0 \mathrm{~Hz}, 2 \mathrm{H}), 7.53-7.39(\mathrm{~m}, 5 \mathrm{H})$, $7.35-7.28(\mathrm{~m}, 2 \mathrm{H}), 7.20-7.13(\mathrm{~m}, 1 \mathrm{H}), 7.12-6.94(\mathrm{~m}, 8 \mathrm{H}), 6.80(\mathrm{~d}, J=$ 
$7.4 \mathrm{~Hz}, 2 \mathrm{H}), 3.84$ (d, $J=13.3 \mathrm{~Hz}, 1 \mathrm{H}), 3.56$ (d, $J=10.1 \mathrm{~Hz}, 1 \mathrm{H}), 3.50$ (d, $J=13.3 \mathrm{~Hz}, 1 \mathrm{H}), 2.89-$ $2.77(\mathrm{~m}, 1 \mathrm{H}), 2.55-2.43(\mathrm{~m}, 1 \mathrm{H}), 1.94-1.75(\mathrm{~m}, 2 \mathrm{H}) .{ }^{13} \mathrm{C} \mathrm{NMR}\left(101 \mathrm{MHz}, \mathrm{CDCl}_{3}\right)$ (one aromatic carbon missing) $\delta 174.5,158.3,137.2,136.8,134.5,131.9,130.1,129.2,128.7,128.6$, $128.3(\mathrm{q}, J=258.6 \mathrm{~Hz}), 128.28,128.1,127.8,127.2,126.8,125.8,120.4,63.6,50.2,40.6,32.1$ (q, $J=28.7 \mathrm{~Hz}), 20.1(\mathrm{q}, J=3.0 \mathrm{~Hz}) .{ }^{19} \mathrm{~F}$ NMR $\left(377 \mathrm{MHz}, \mathrm{CDCl}_{3}\right) \delta-65.75(\mathrm{t}, J=10.8 \mathrm{~Hz})$.

HPLC analysis: Daicel CHIRALPAK OD-H, $n$-hexane $/ i-\mathrm{PrOH}=95 / 5$, flow rate $=0.8 \mathrm{~mL} / \mathrm{min}, \lambda$ $=254 \mathrm{~nm}$, retention time: $t_{\text {minor }}=6.11 \mathrm{~min}, t_{\text {major }}=6.56 \mathrm{~min}$.

HRMS (ESI) $m / z$ : calcd for $\mathrm{C}_{32} \mathrm{H}_{28} \mathrm{~F}_{3} \mathrm{~N}_{2} \mathrm{O}[\mathrm{M}+\mathrm{H}]^{+}$513.2154, found: 513.2145.

(S)-4-Benzyl-1,3-diphenyl-4-((1S,2S,3S)-4,4,4-trifluoro-2,3-dihydroxy-1-phenylbutyl)-1 $H$-pyrazol $-5(4 H)$-one $(7 \mathbf{a})$

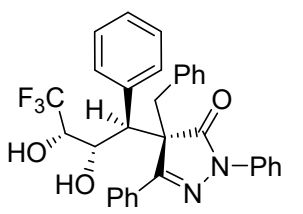

Column chromatography elution: petroleum ether/ethyl acetate $=10: 1(\mathrm{v} / \mathrm{v})$, white solid, mp $234-235{ }^{\circ} \mathrm{C}, 106.7 \mathrm{mg}, 49 \%$ yield, $>20: 1 \mathrm{dr}, 95 \%$ ee.

$[\alpha]_{\mathrm{D}}^{20}=+31.11\left(\mathrm{c} 0.27, \mathrm{CH}_{2} \mathrm{Cl}_{2}\right)$.

${ }^{1} \mathrm{H}$ NMR $\left(400 \mathrm{MHz}, \mathrm{CDCl}_{3}\right) \delta 7.77-7.71(\mathrm{~m}, 2 \mathrm{H}), 7.56-7.49(\mathrm{~m}, 2 \mathrm{H})$, $7.43-7.33(\mathrm{~m}, 5 \mathrm{H}), 7.26-7.21(\mathrm{~m}, 1 \mathrm{H}), 7.09-7.00(\mathrm{~m}, 5 \mathrm{H}), 7.00-6.92(\mathrm{~m}, 3 \mathrm{H}), 6.80(\mathrm{~d}, J=$ $7.1 \mathrm{~Hz}, 2 \mathrm{H}), 5.30(\mathrm{~d}, J=5.0 \mathrm{~Hz}, 1 \mathrm{H}), 4.83(\mathrm{dd}, J=10.3,5.0 \mathrm{~Hz}, 1 \mathrm{H}), 4.33(\mathrm{~d}, J=13.7 \mathrm{~Hz}, 1 \mathrm{H})$, $3.92(\mathrm{~d}, J=10.3 \mathrm{~Hz}, 1 \mathrm{H}), 3.67$ (d, $J=13.7 \mathrm{~Hz}, 1 \mathrm{H}), 3.60-3.51(\mathrm{~m}, 1 \mathrm{H}), 3.40$ (d, $J=11.6 \mathrm{~Hz}$, 1H). ${ }^{13} \mathrm{C} \mathrm{NMR}\left(101 \mathrm{MHz}, \mathrm{CDCl}_{3}\right.$ ) (two aromatic carbons missing) $\delta 176.1,159.9,136.9,136.7$, $134.2,131.3,130.3,129.5,128.9,128.6,128.5,128.1,127.4,126.8,126.5,124.5$ (q, $J=283.7$ $\mathrm{Hz}), 120.7,70.7,69.6(\mathrm{q}, J=30.4 \mathrm{~Hz}), 64.5,53.1,43.5 .{ }^{19} \mathrm{~F} \mathrm{NMR}\left(377 \mathrm{MHz}, \mathrm{CDCl}_{3}\right) \delta-77.05(\mathrm{~d}$, $J=4.5 \mathrm{~Hz}$ ).

HPLC analysis: Daicel CHIRALPAK OD-H, $n$-hexane $/ i$-PrOH $=9 / 1$, flow rate $=0.8 \mathrm{~mL} / \mathrm{min}, \lambda=$ $254 \mathrm{~nm}$, retention time: $t_{\text {minor }}=7.51 \mathrm{~min}, t_{\text {major }}=15.90 \mathrm{~min}$.

HRMS (ESI) $m / z$ : calcd for $\mathrm{C}_{32} \mathrm{H}_{28} \mathrm{~F}_{3} \mathrm{~N}_{2} \mathrm{O}_{3}[\mathrm{M}+\mathrm{H}]^{+}$545.2052, found: 545.2052. 
10. Copies of ${ }^{1} \mathrm{H},{ }^{13} \mathrm{C}$ and ${ }^{19} \mathrm{~F}$ NMR spectrum
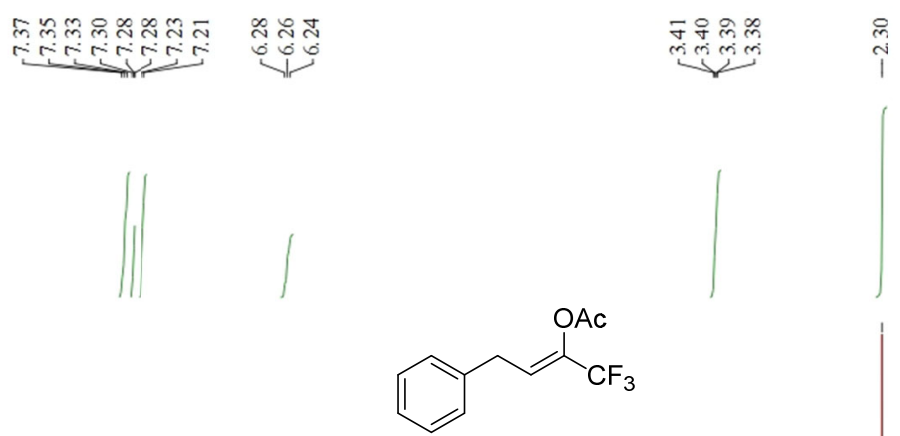

2a ${ }^{1} \mathrm{H} \mathrm{NMR}\left(\mathrm{CDCl}_{3}, 400 \mathrm{MHz}\right)$

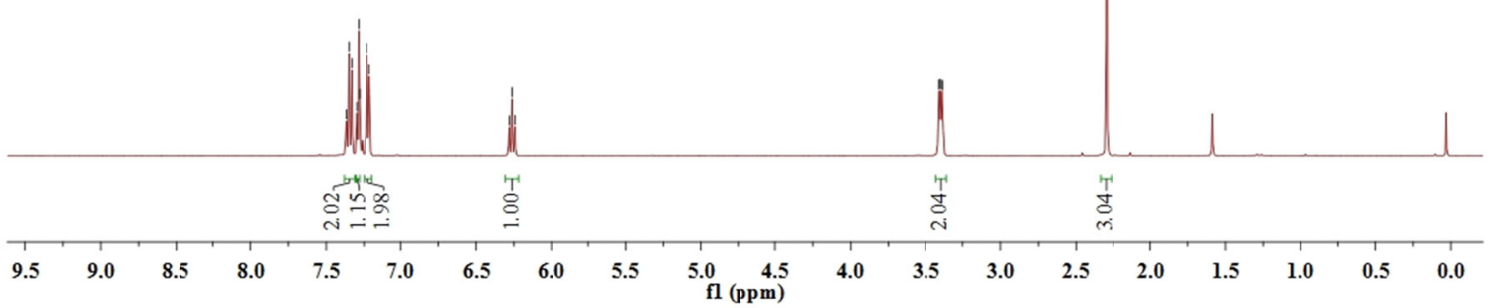

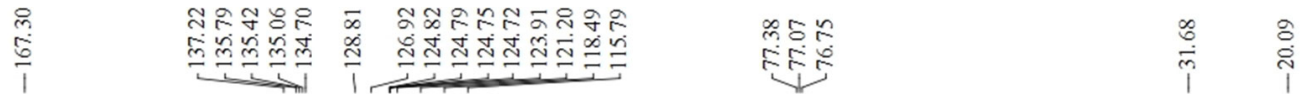

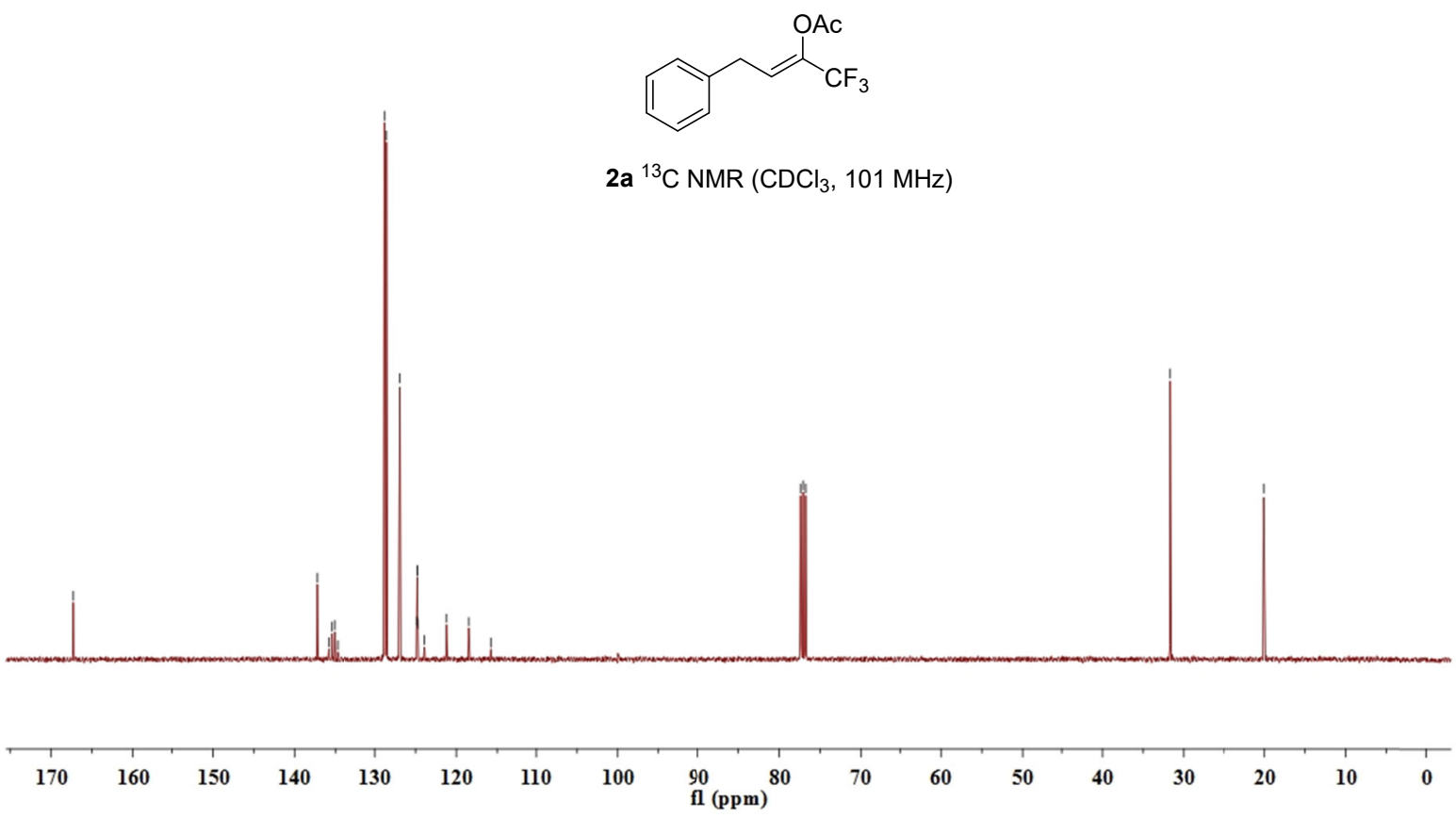


<smiles>CC(=O)OC(=CCc1ccccc1)C(F)(F)F</smiles>

2a ${ }^{19} \mathrm{~F} \mathrm{NMR}\left(\mathrm{CDCl}_{3}, 470 \mathrm{MHz}\right)$
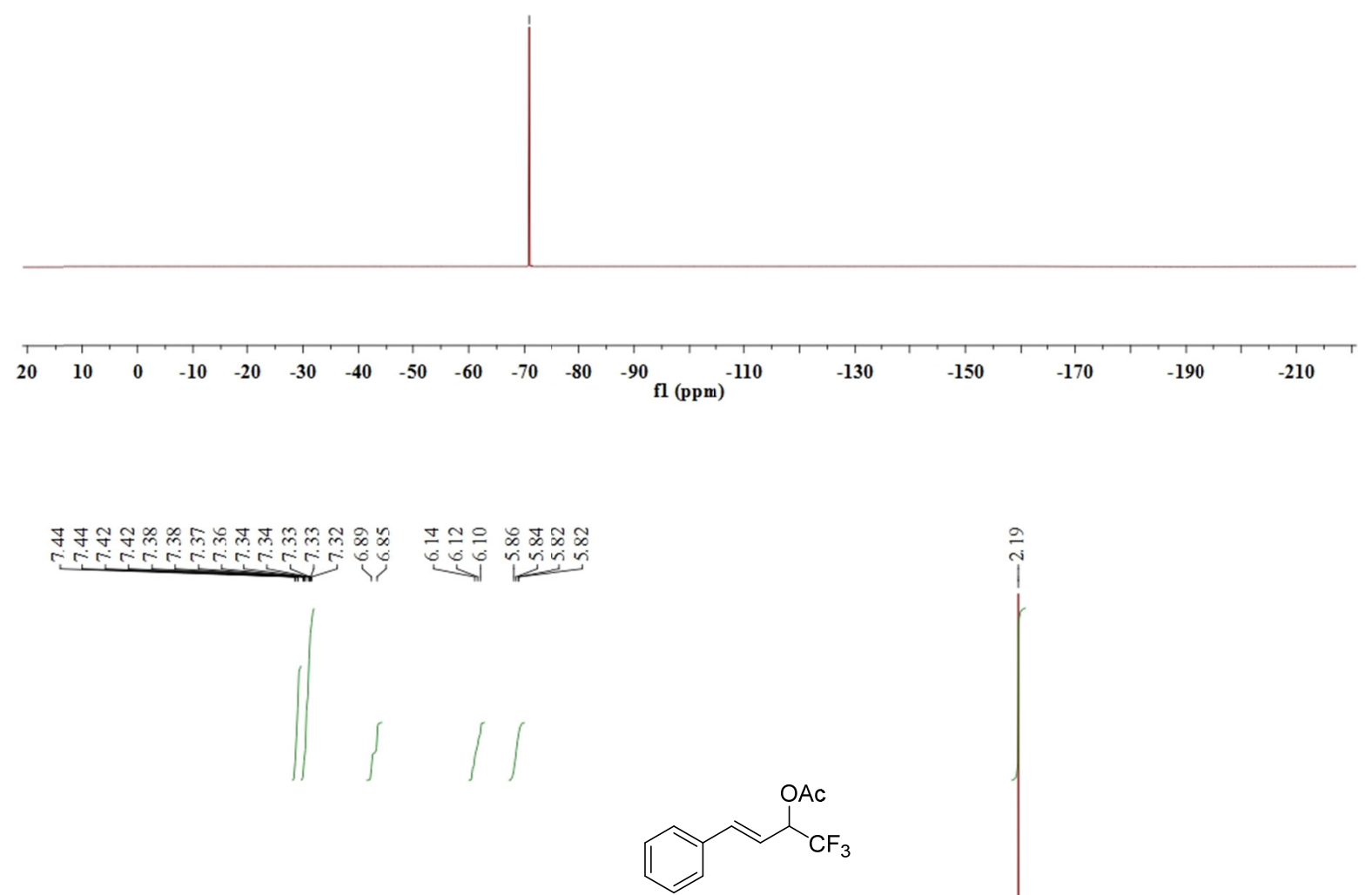

2a' ${ }^{1} \mathrm{H}$ NMR $\left(\mathrm{CDCl}_{3}, 400 \mathrm{MHz}\right)$

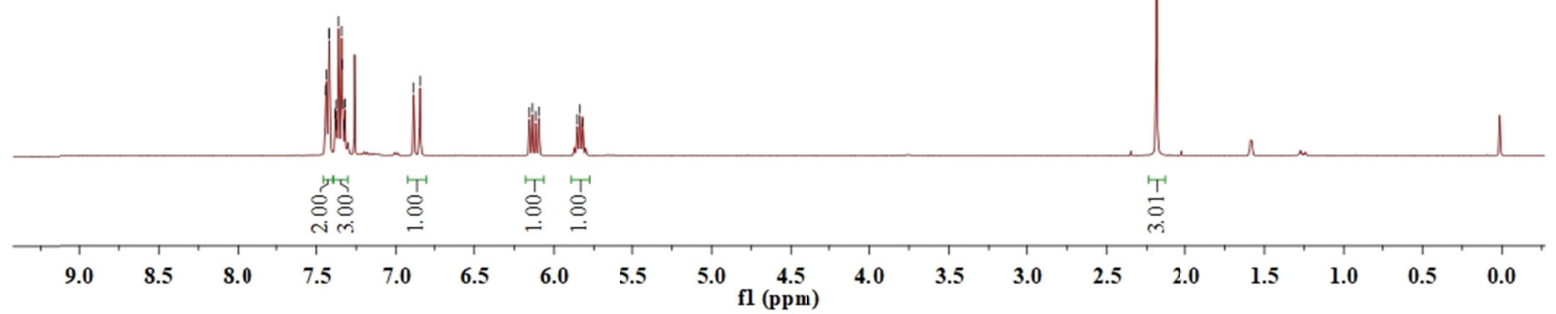




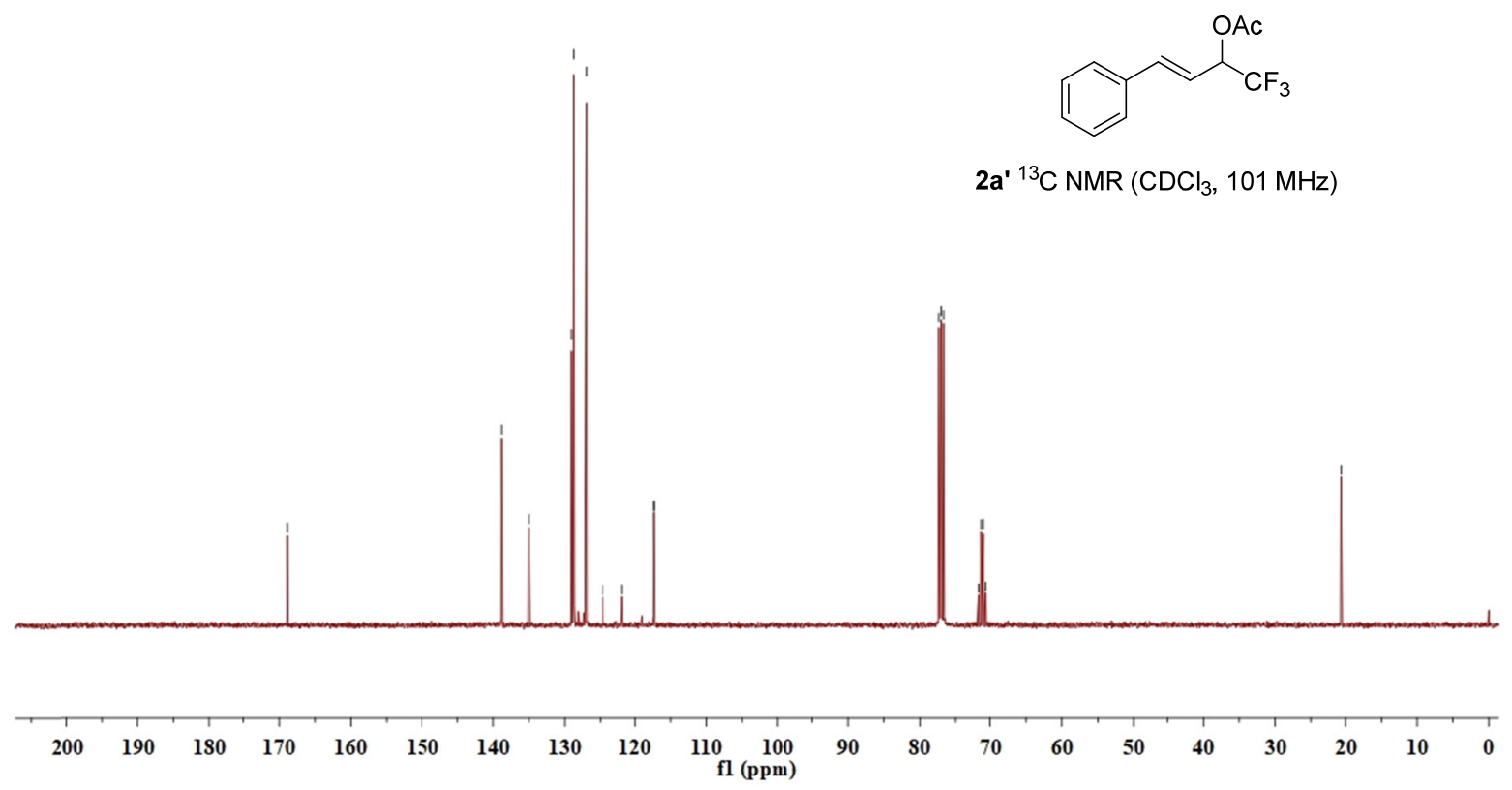

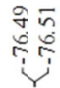

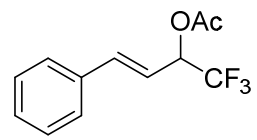

$2 \mathbf{a}^{19} \mathrm{~F} \mathrm{NMR}\left(\mathrm{CDCl}_{3}, 377 \mathrm{MHz}\right)$

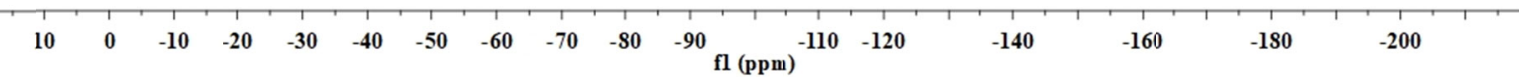



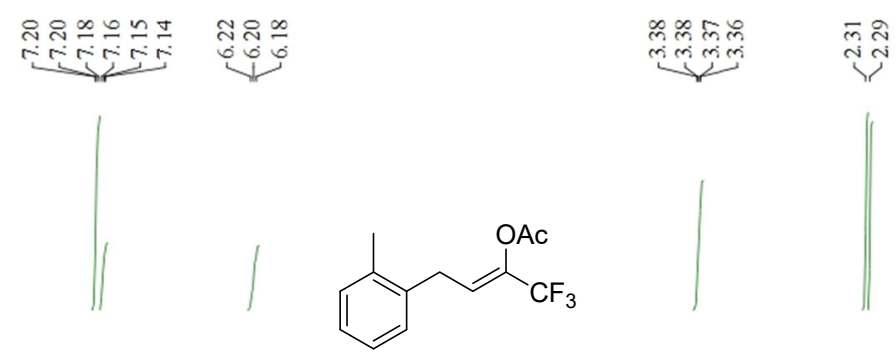

2b ${ }^{1} \mathrm{H}$ NMR $\left(\mathrm{CDCl}_{3}, 400 \mathrm{MHz}\right)$
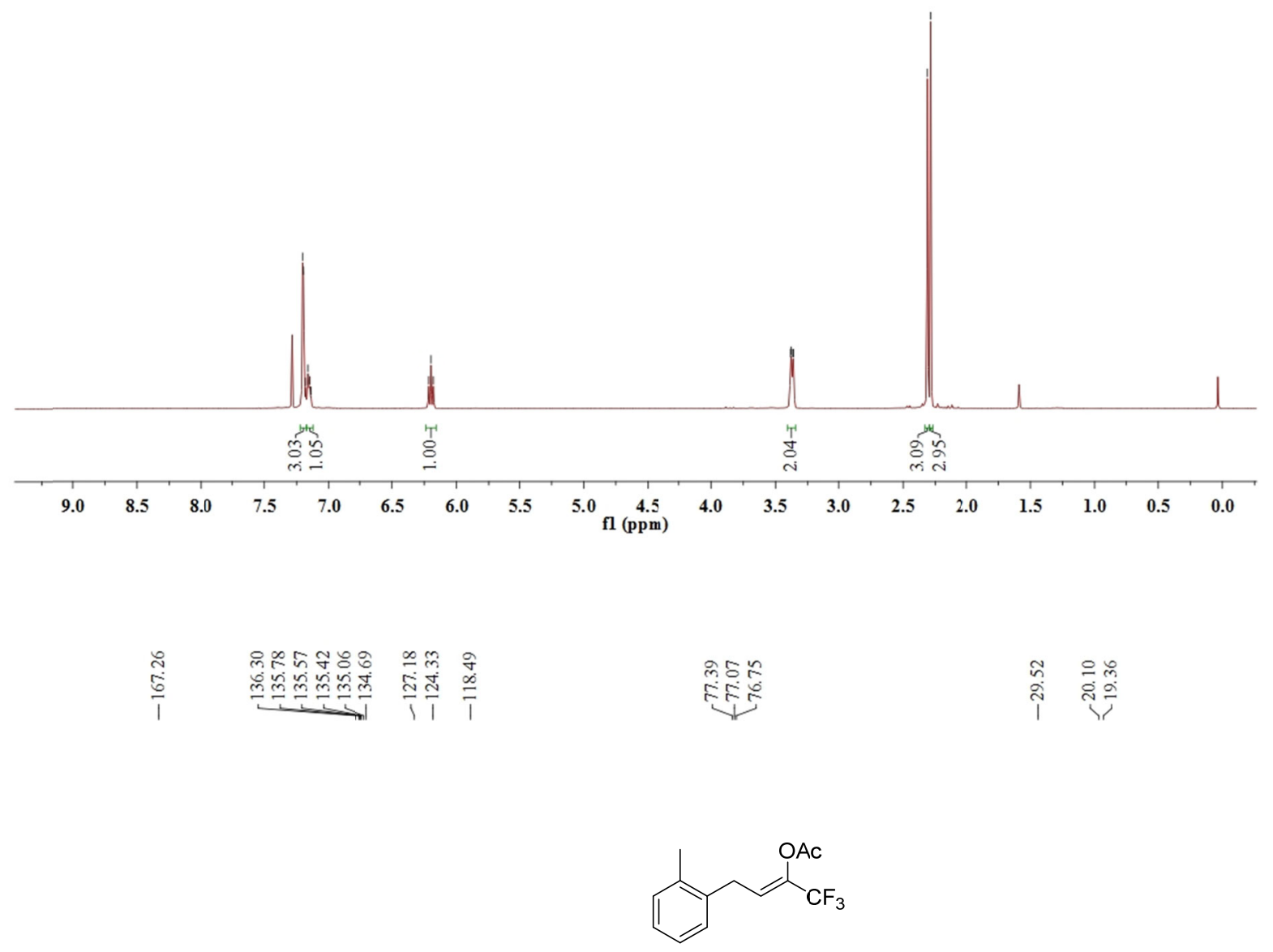

2b ${ }^{13} \mathrm{C}$ NMR $\left(\mathrm{CDCl}_{3}, 101 \mathrm{MHz}\right)$

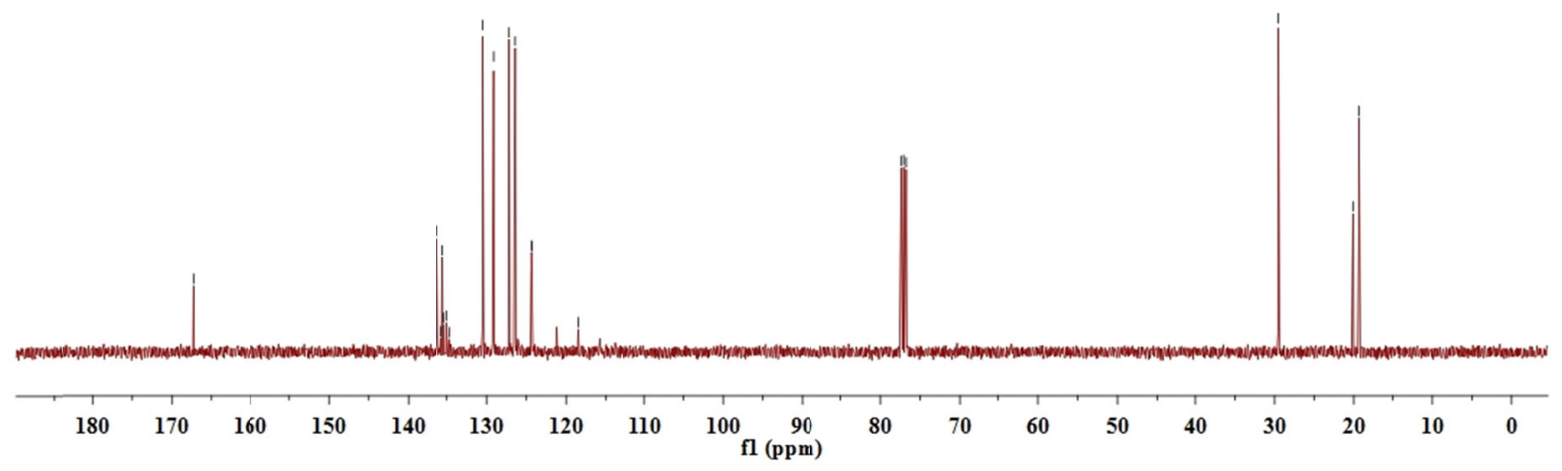


<smiles>CC(=O)OC(=CCc1ccccc1C)C(F)(F)F</smiles>

2b ${ }^{19} \mathrm{~F} \mathrm{NMR}\left(\mathrm{CDCl}_{3}, 470 \mathrm{MHz}\right)$
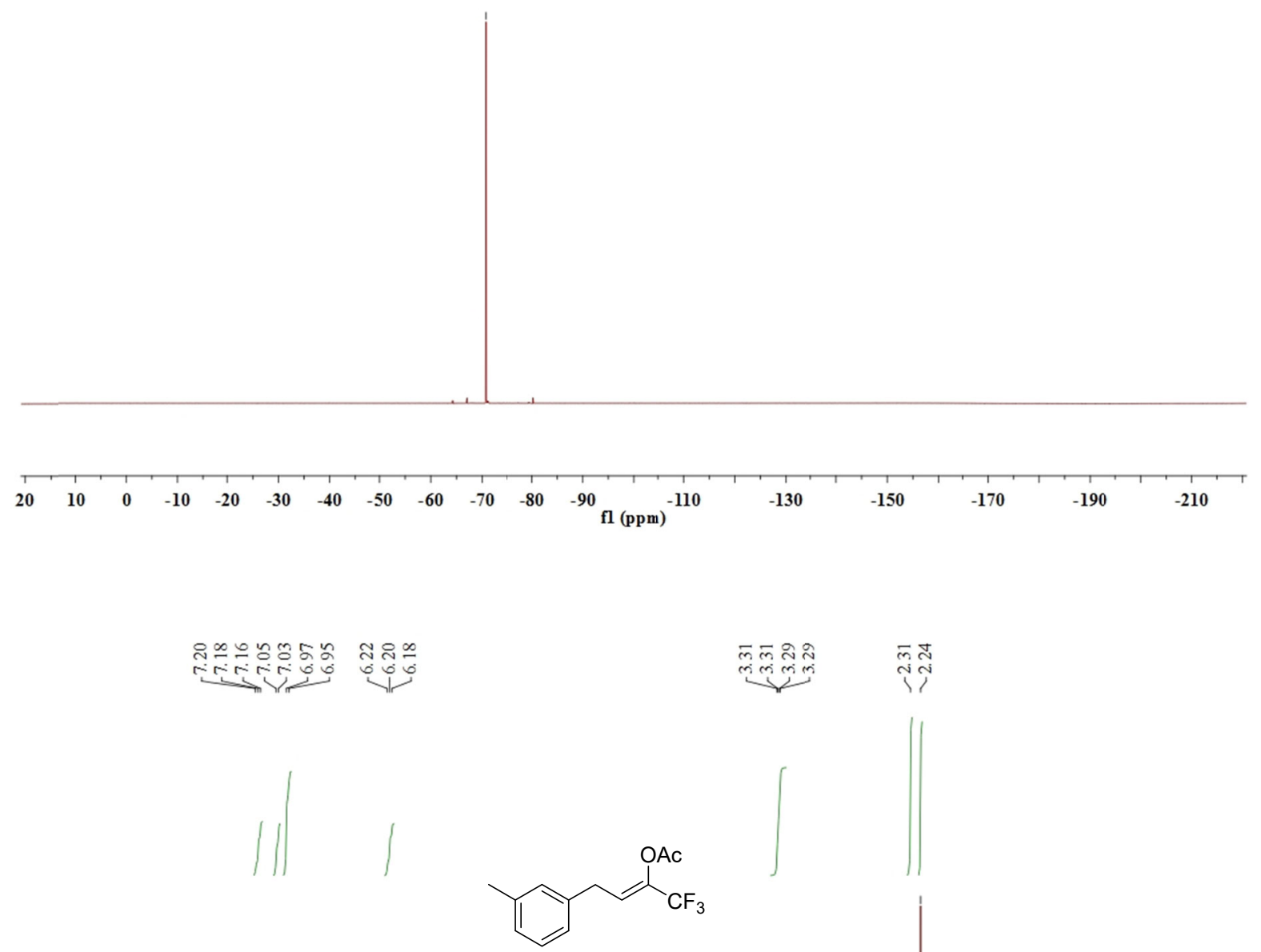

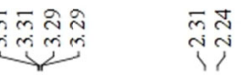

2c ${ }^{1} \mathrm{H}$ NMR $\left(\mathrm{CDCl}_{3}, 400 \mathrm{MHz}\right)$

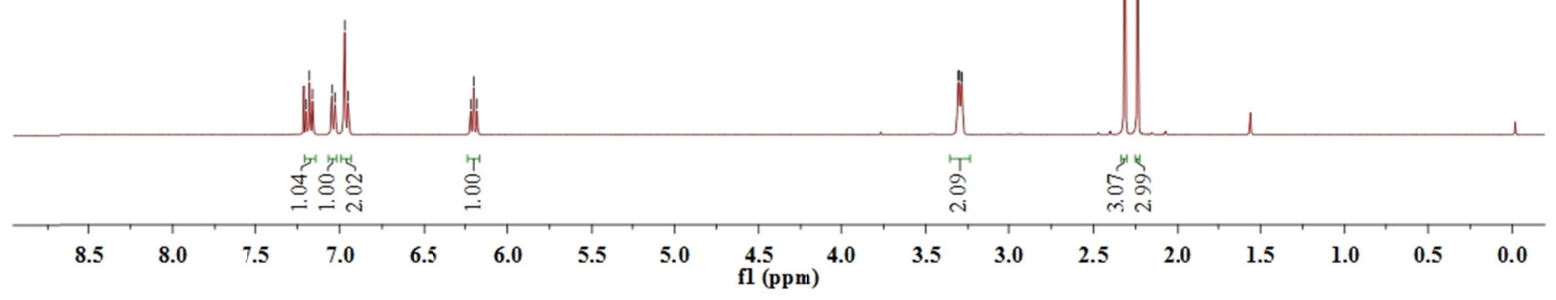




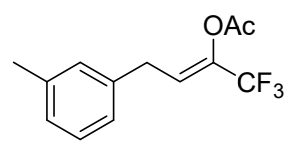

$2{ }^{13} \mathrm{C} \mathrm{NMR}\left(\mathrm{CDCl}_{3}, 101 \mathrm{MHz}\right)$

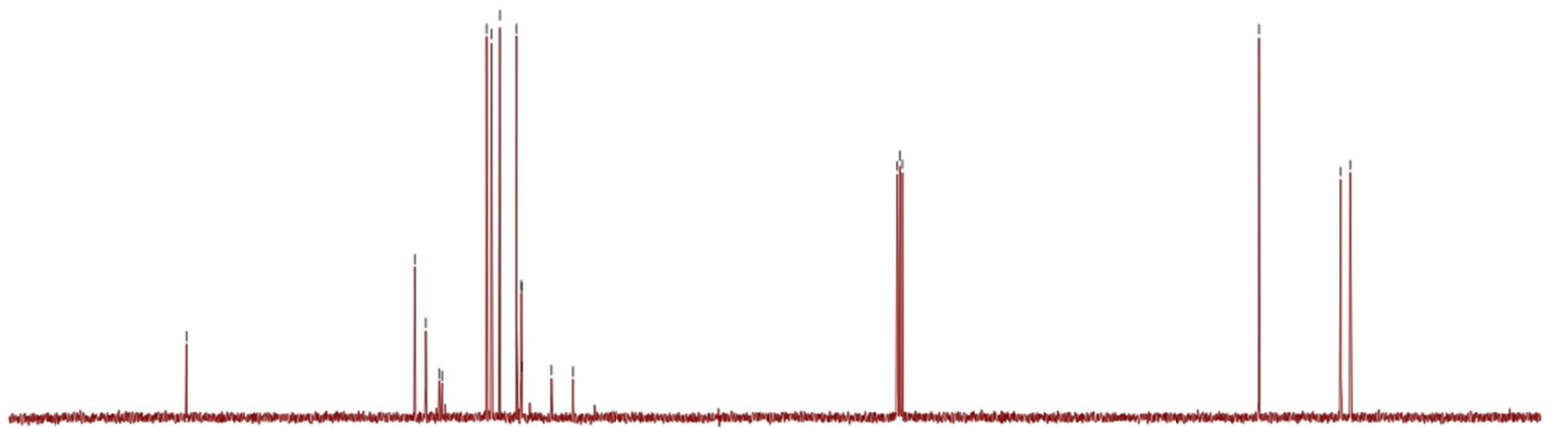

$\begin{array}{lllllllllllllllllll}180 & 170 & 160 & 150 & 140 & 130 & 120 & 110 & 100 & 90 & 80 & 70 & 60 & 50 & 40 & 30 & 20 & 10 & 0\end{array}$

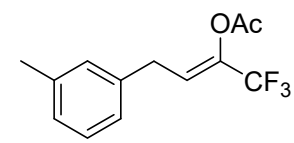

2c ${ }^{19} \mathrm{~F} \mathrm{NMR}\left(\mathrm{CDCl}_{3}, 377 \mathrm{MHz}\right)$

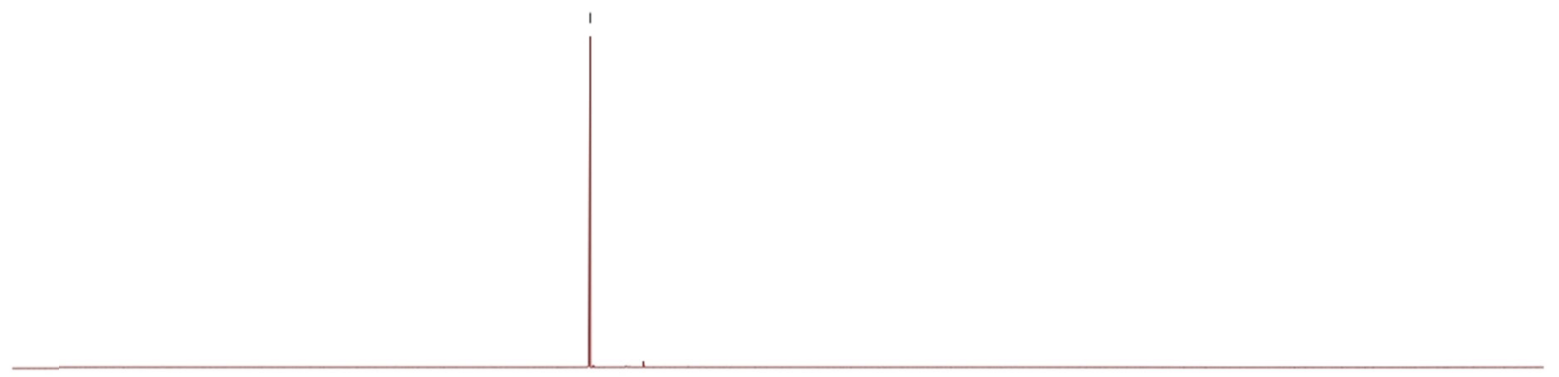




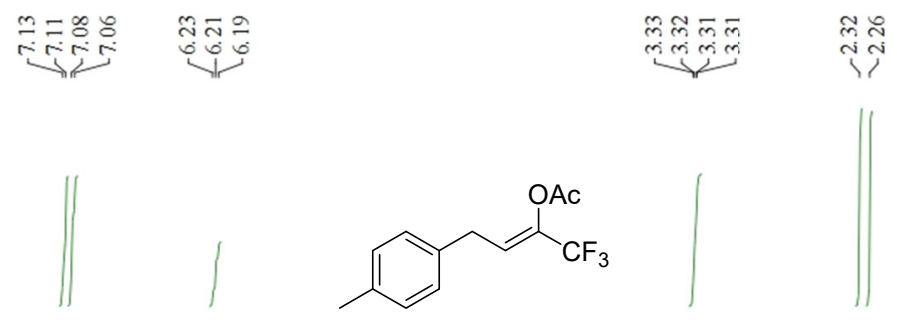

2d ${ }^{1} \mathrm{H}$ NMR $\left(\mathrm{CDCl}_{3}, 400 \mathrm{MHz}\right)$

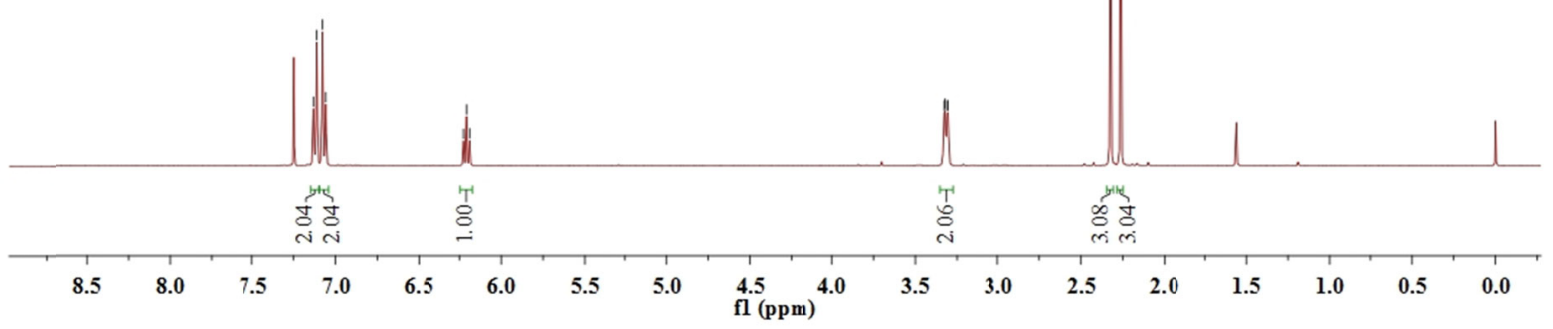

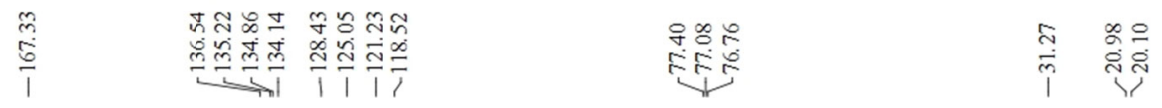

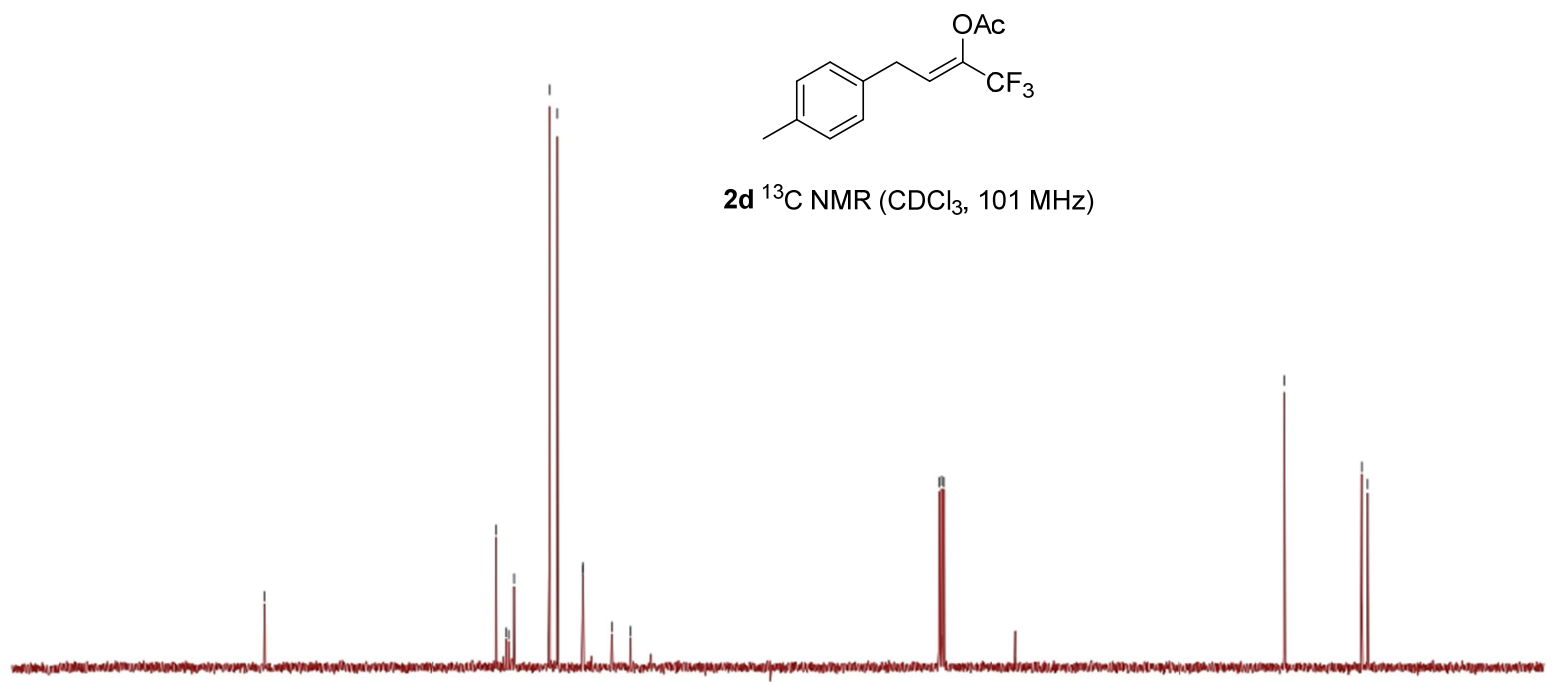

$2{ }^{13} \mathrm{C} \mathrm{NMR}\left(\mathrm{CDCl}_{3}, 101 \mathrm{MHz}\right)$

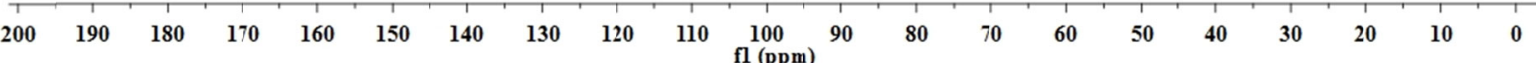




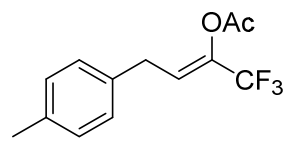

2d ${ }^{19} \mathrm{~F} \mathrm{NMR}\left(\mathrm{CDCl}_{3}, 377 \mathrm{MHz}\right)$

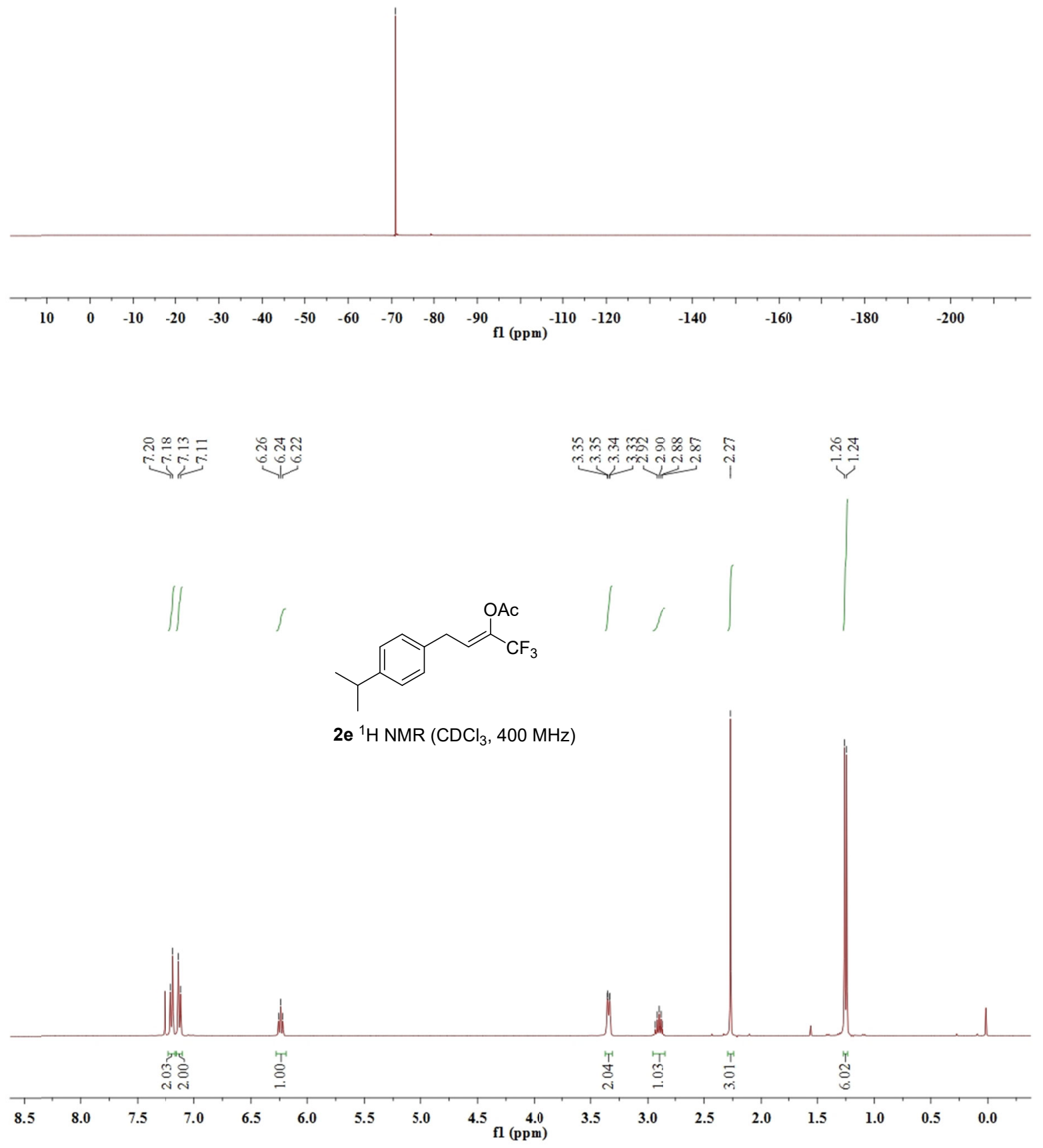



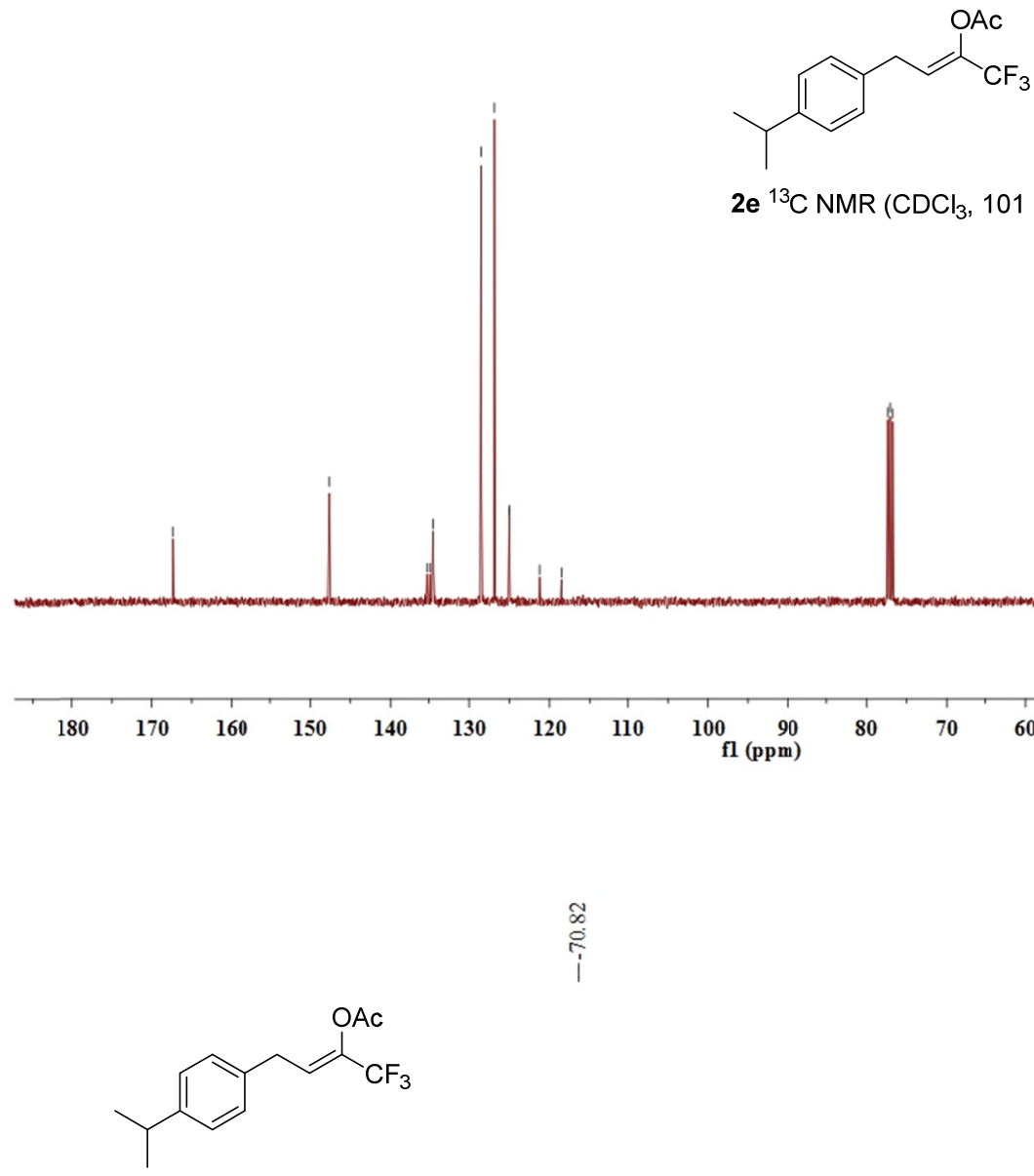

$2 e^{13} \mathrm{C} \mathrm{NMR}\left(\mathrm{CDCl}_{3}, 101 \mathrm{MHz}\right)$

$2 e^{19} \mathrm{~F} \mathrm{NMR}\left(\mathrm{CDCl}_{3}, 377 \mathrm{MHz}\right)$

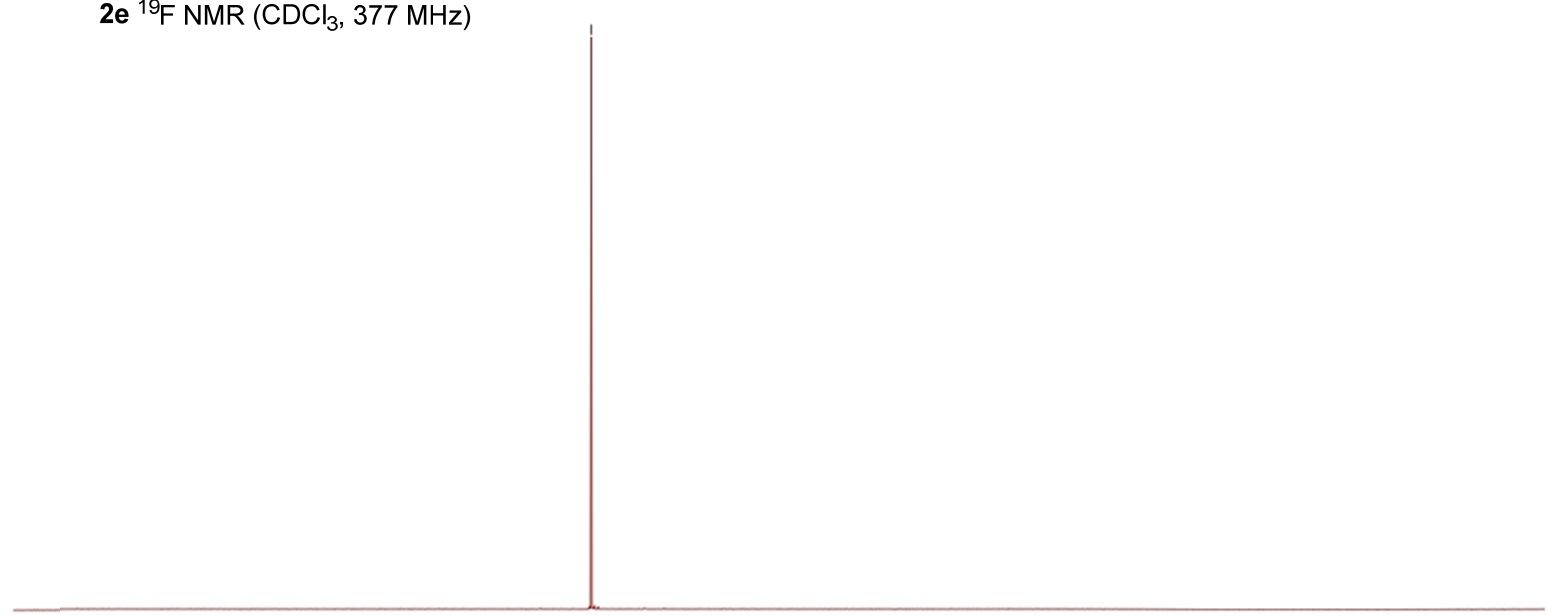




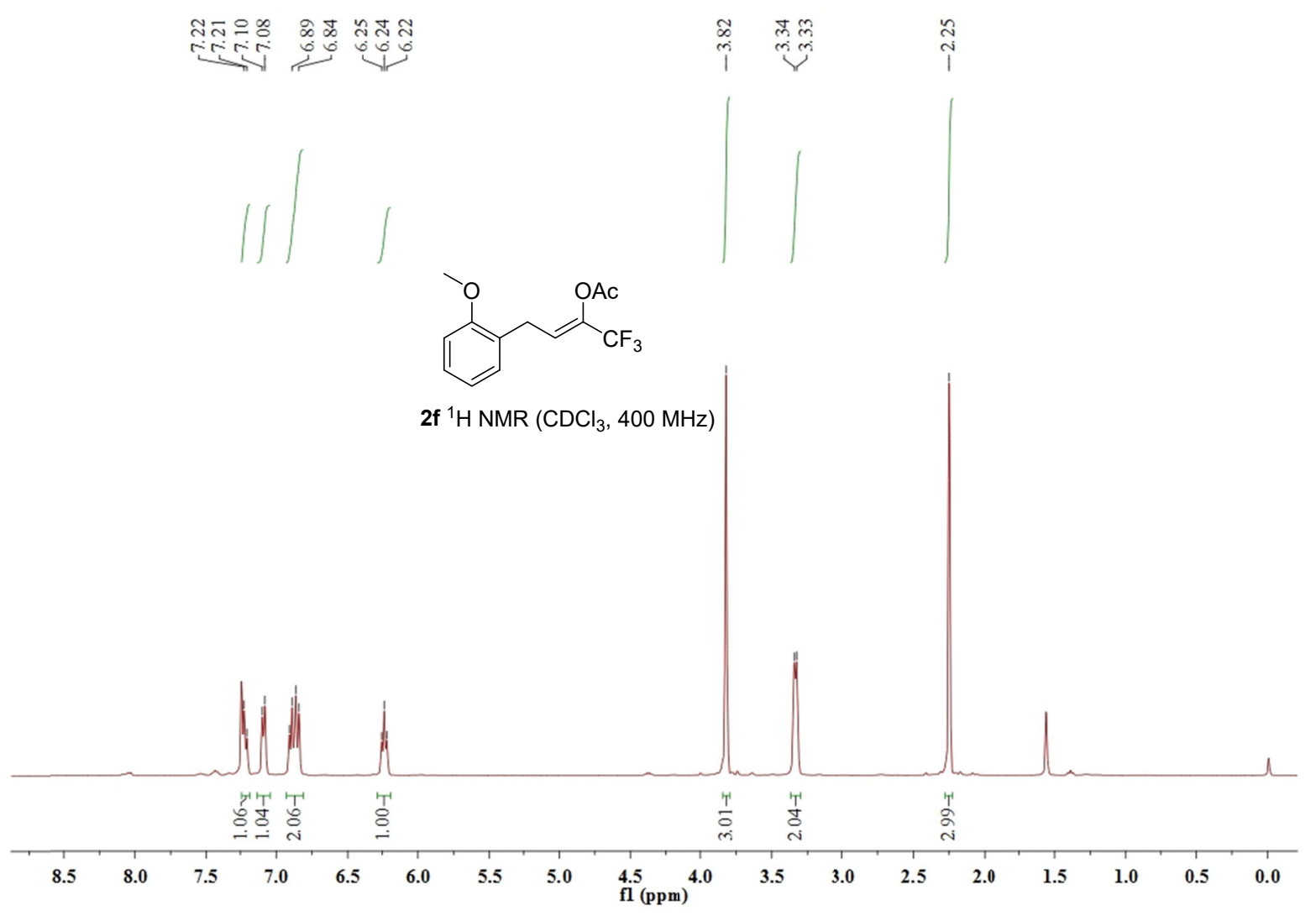

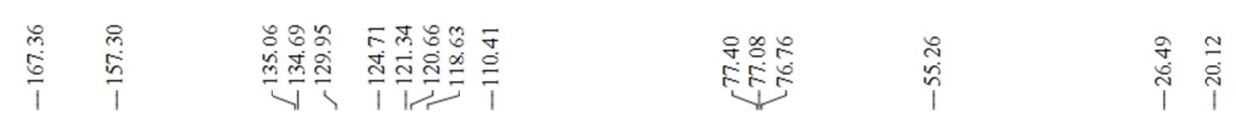<smiles>COc1ccccc1CC=C(C(C)=O)C(F)(F)F</smiles>

$2{ }^{13} \mathrm{C} \mathrm{NMR}\left(\mathrm{CDCl}_{3}, 101 \mathrm{MHz}\right)$

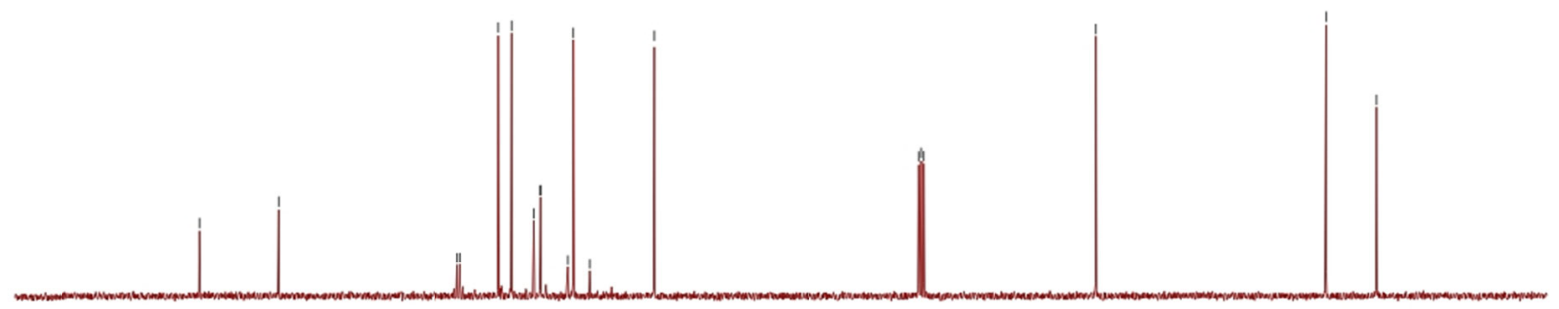

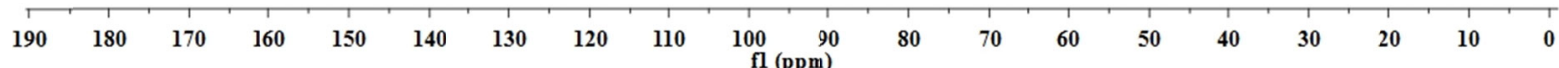




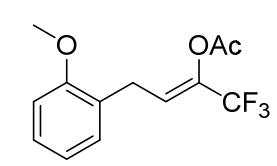

$\stackrel{8}{\circ}$

2f ${ }^{19} \mathrm{~F} \mathrm{NMR}\left(\mathrm{CDCl}_{3}, 377 \mathrm{MHz}\right)$

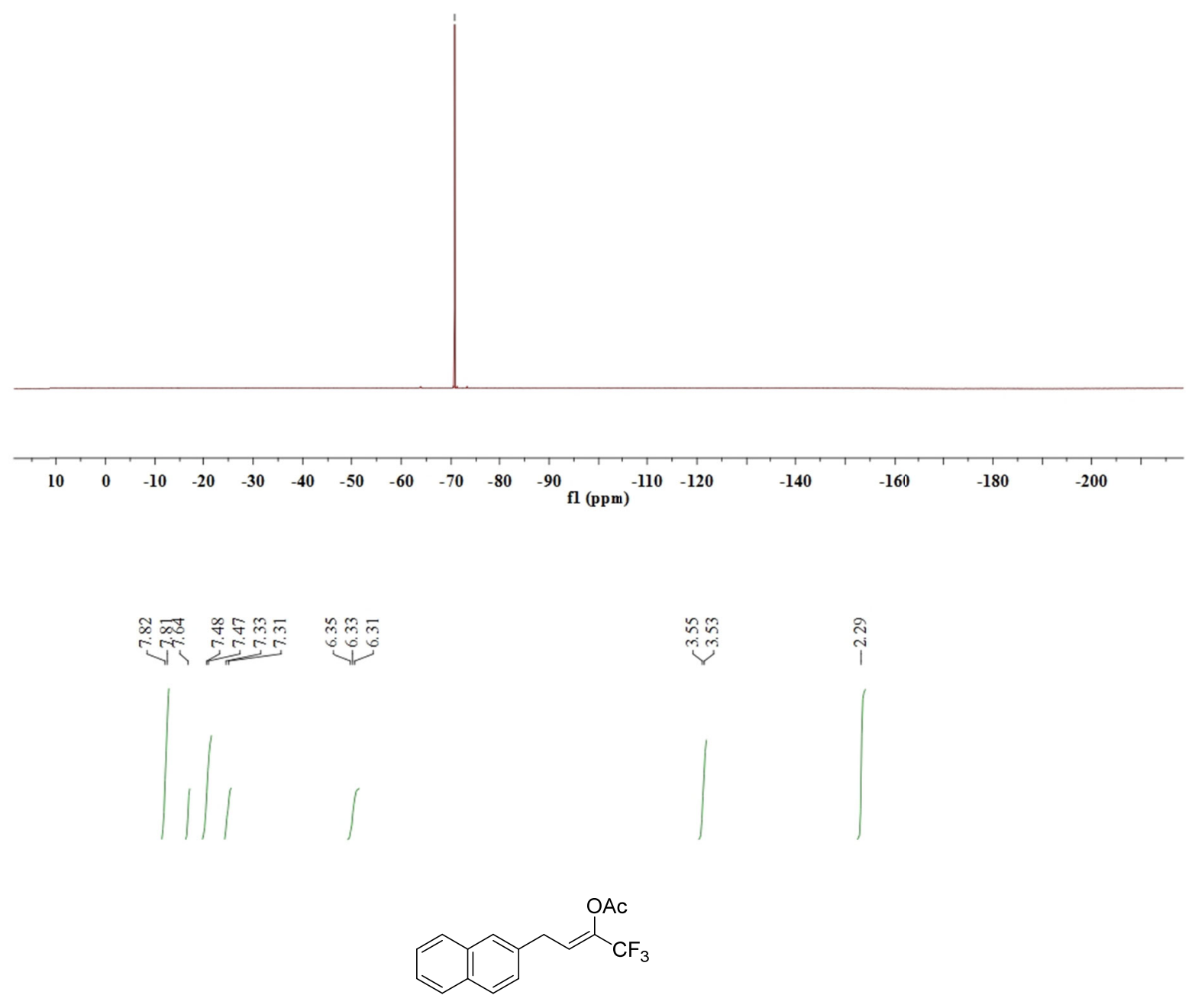

2g ${ }^{1} \mathrm{H} \mathrm{NMR}\left(\mathrm{CDCl}_{3}, 400 \mathrm{MHz}\right)$

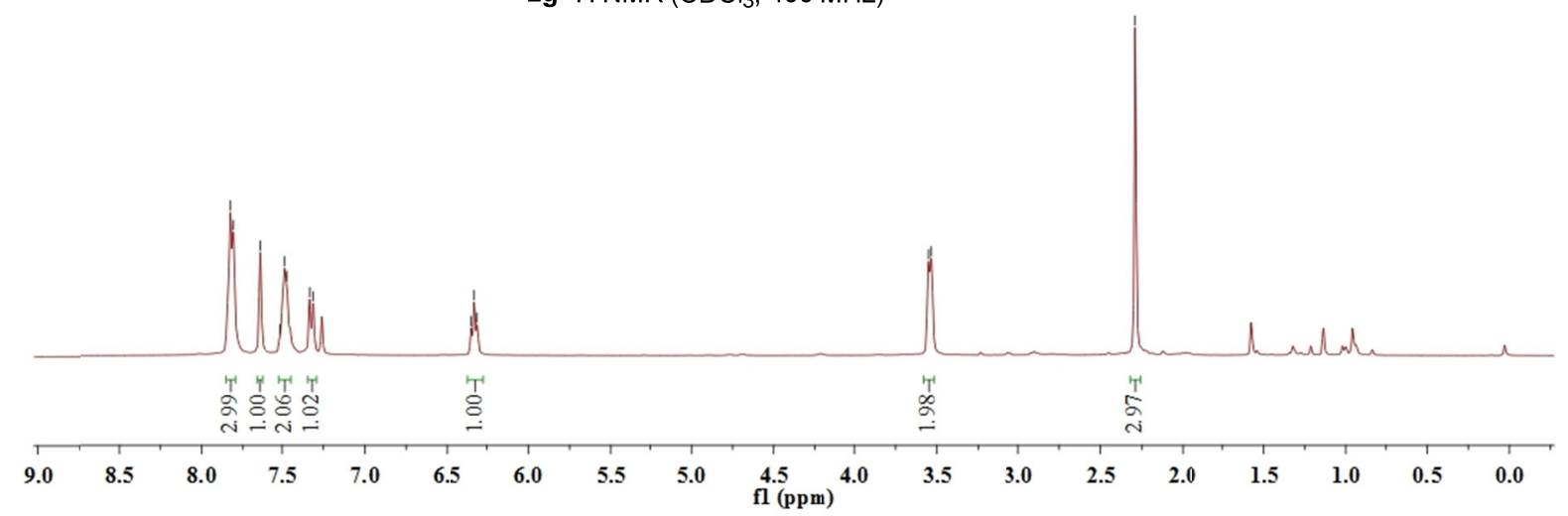




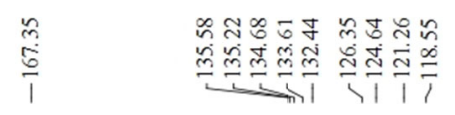

实

$\begin{array}{ll}\infty & \frac{\infty}{\infty} \\ \frac{\infty}{1} & \stackrel{0}{1}\end{array}$

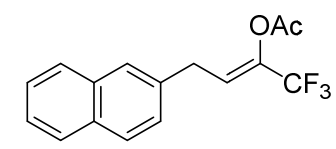

2g ${ }^{13} \mathrm{C} \mathrm{NMR}\left(\mathrm{CDCl}_{3}, 101 \mathrm{MHz}\right)$
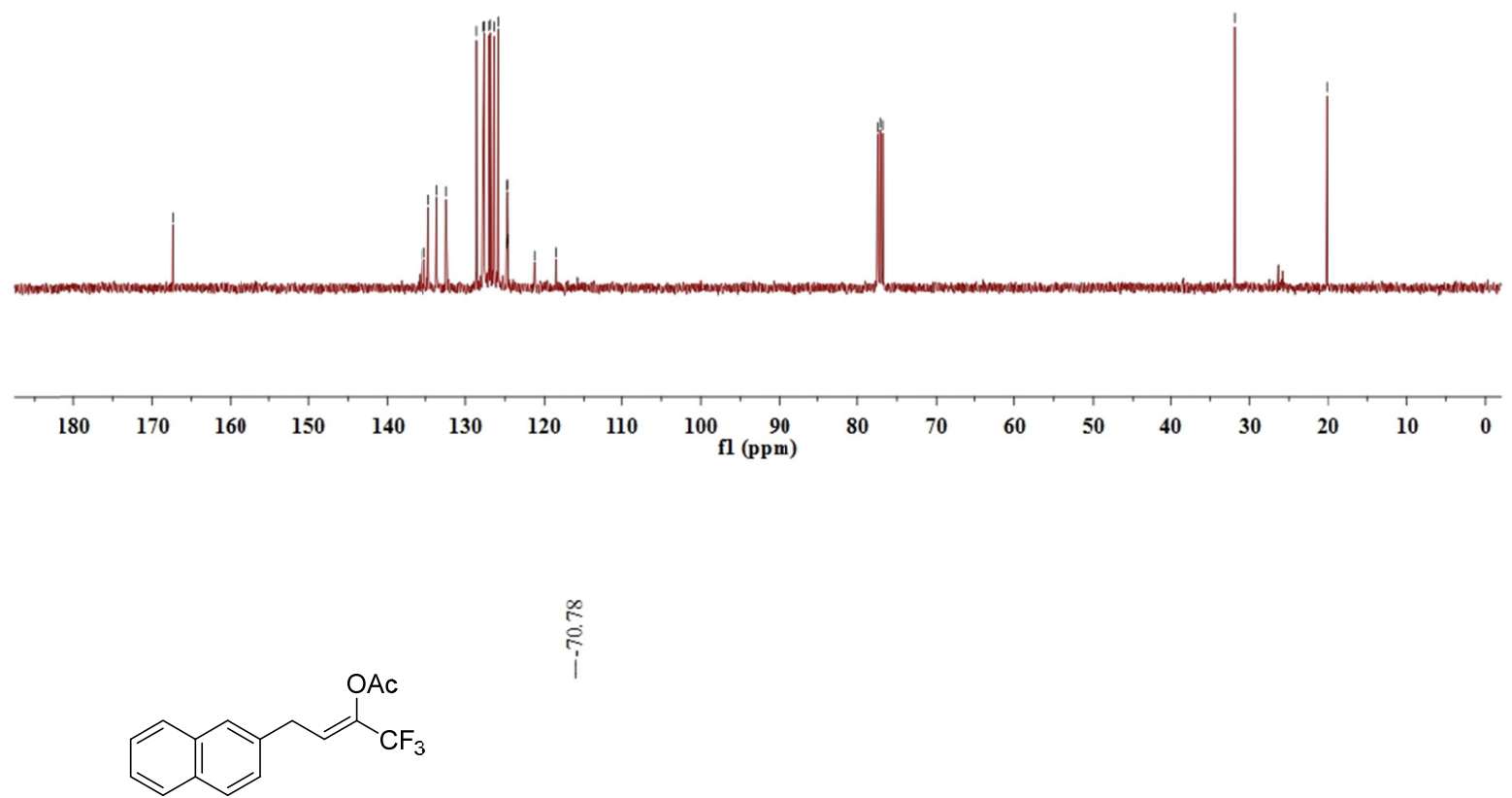

$\stackrel{\infty}{\stackrel{\infty}{i}}$

2g ${ }^{19} \mathrm{~F} \mathrm{NMR}\left(\mathrm{CDCl}_{3}, 377 \mathrm{MHz}\right)$

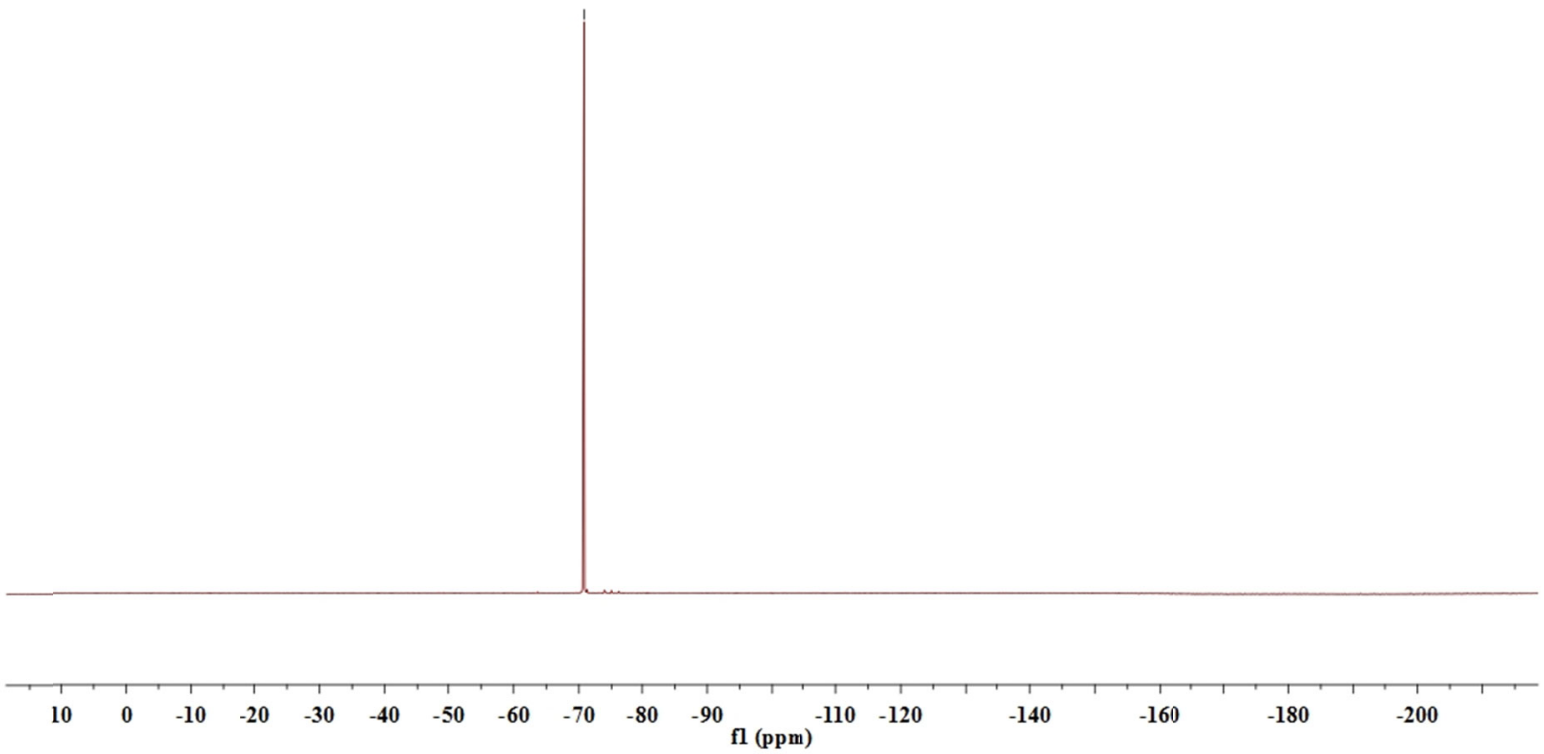

S38 
<smiles>CC(=O)OC(=CCc1ccc(-c2ccccc2)cc1)C(F)(F)F</smiles>

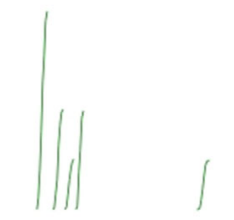

2h ${ }^{1} \mathrm{H}$ NMR $\left(\mathrm{CDCl}_{3}, 400 \mathrm{MHz}\right)$
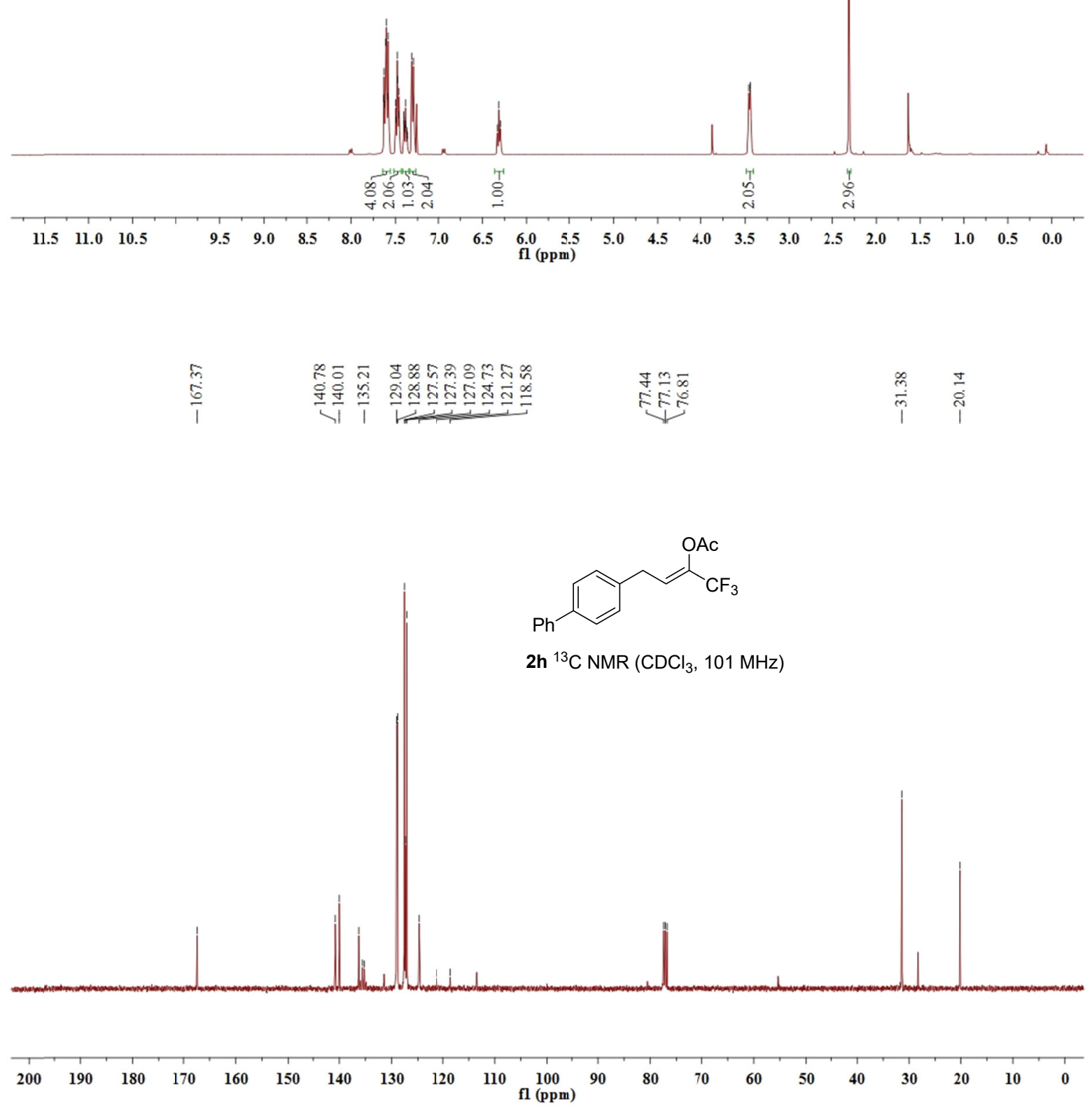


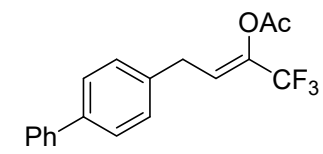

2h $\left.{ }^{19} \mathrm{~F} \mathrm{NMR} \mathrm{(} \mathrm{CDCl}_{3}, 377 \mathrm{MHz}\right)$

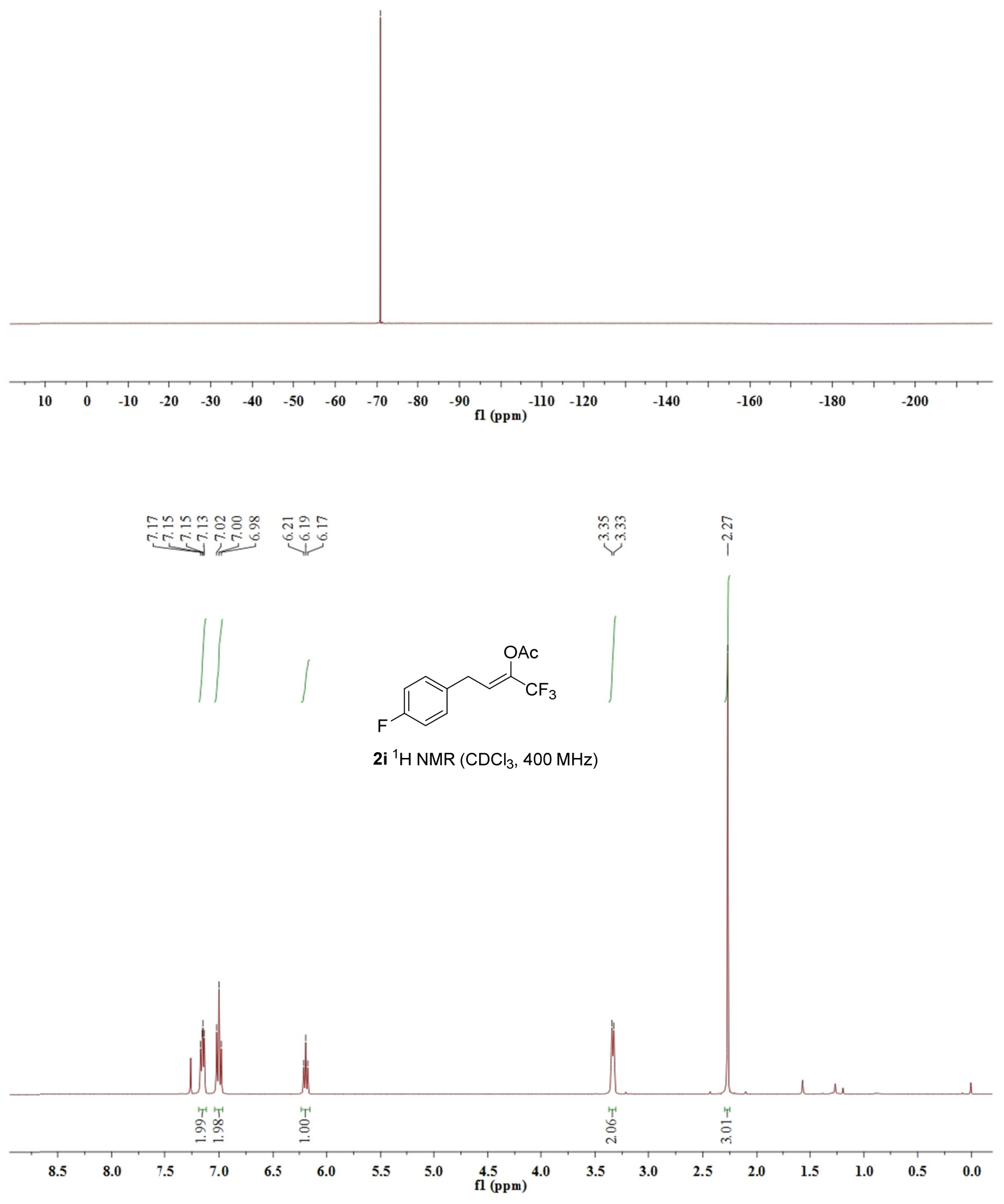




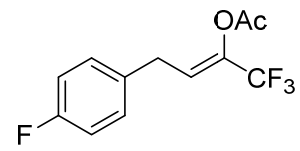

$2 \mathbf{i}{ }^{13} \mathrm{C}$ NMR $\left(\mathrm{CDCl}_{3}, 101 \mathrm{MHz}\right)$
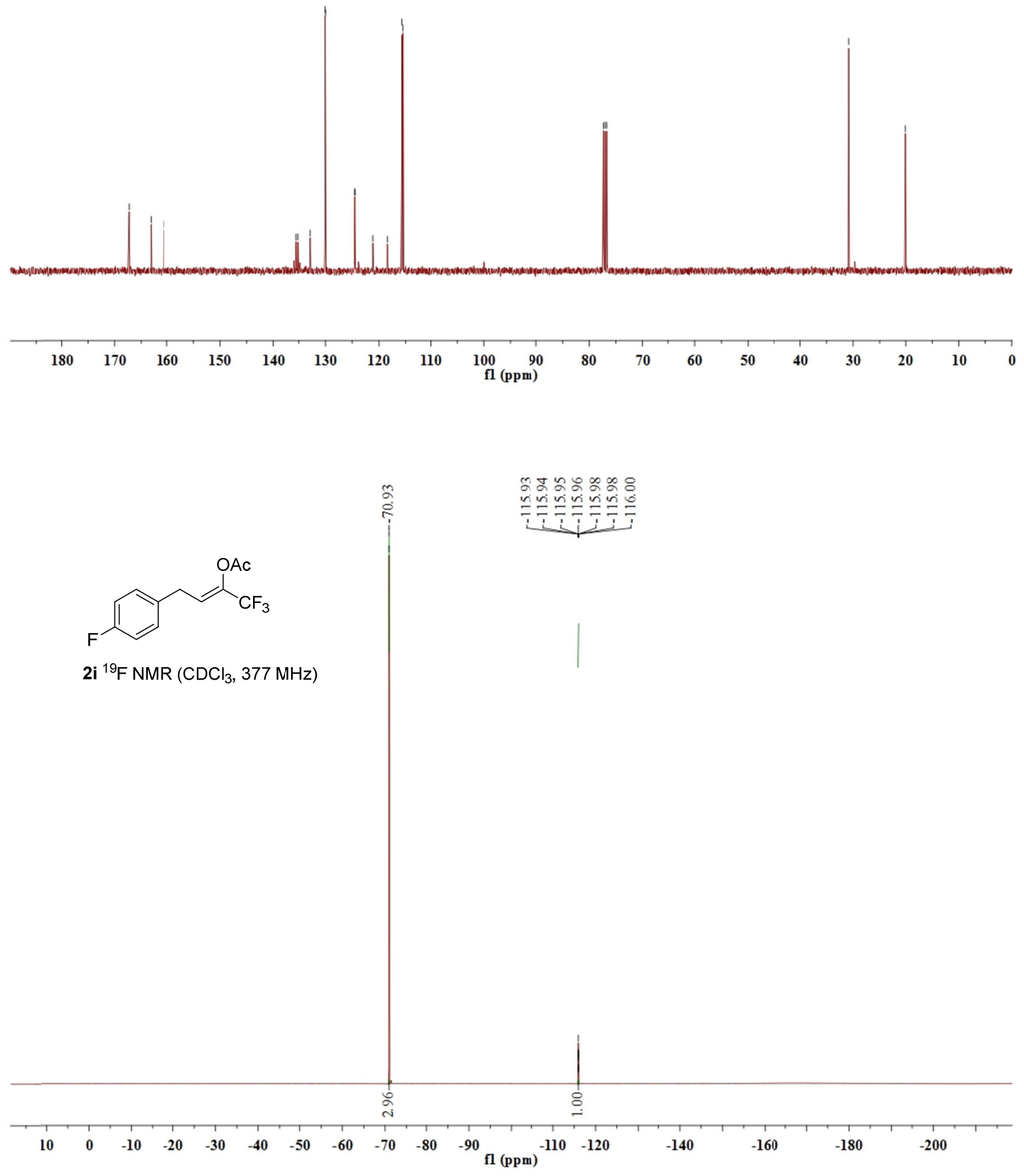


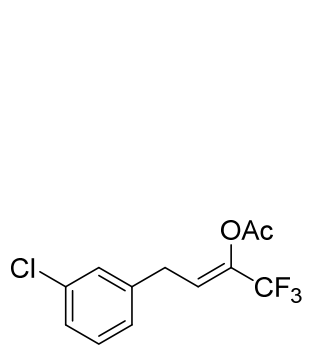

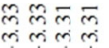

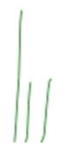<smiles>CCCC</smiles>

2j ${ }^{1} \mathrm{H}$ NMR $\left(\mathrm{CDCl}_{3}, 400 \mathrm{MHz}\right)$
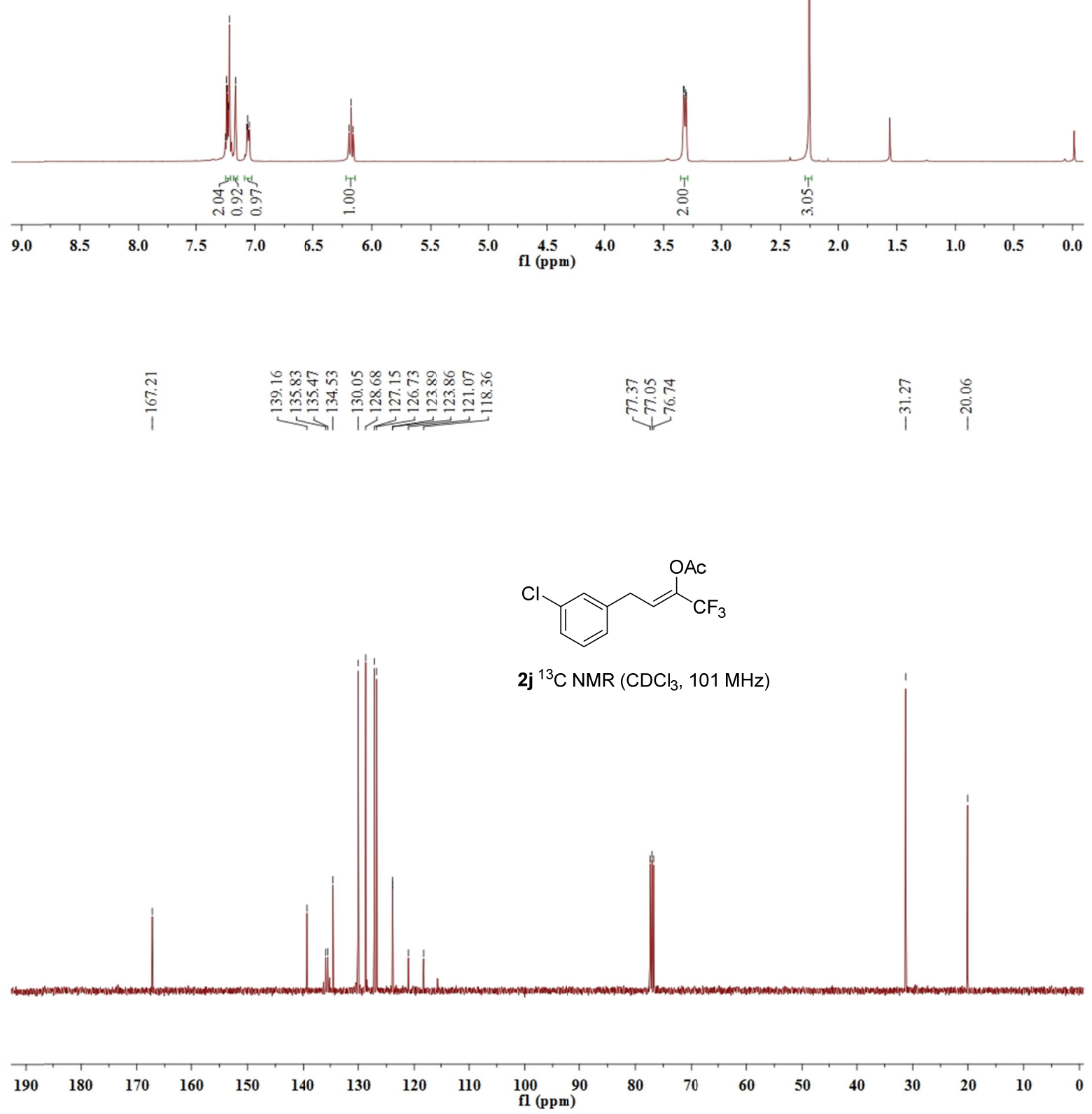
<smiles>COC(=O)C(F)(F)C(=CCc1cccc(Cl)c1)C(F)(F)F</smiles>

2j ${ }^{19} \mathrm{~F} \mathrm{NMR}\left(\mathrm{CDCl}_{3}, 377 \mathrm{MHz}\right)$

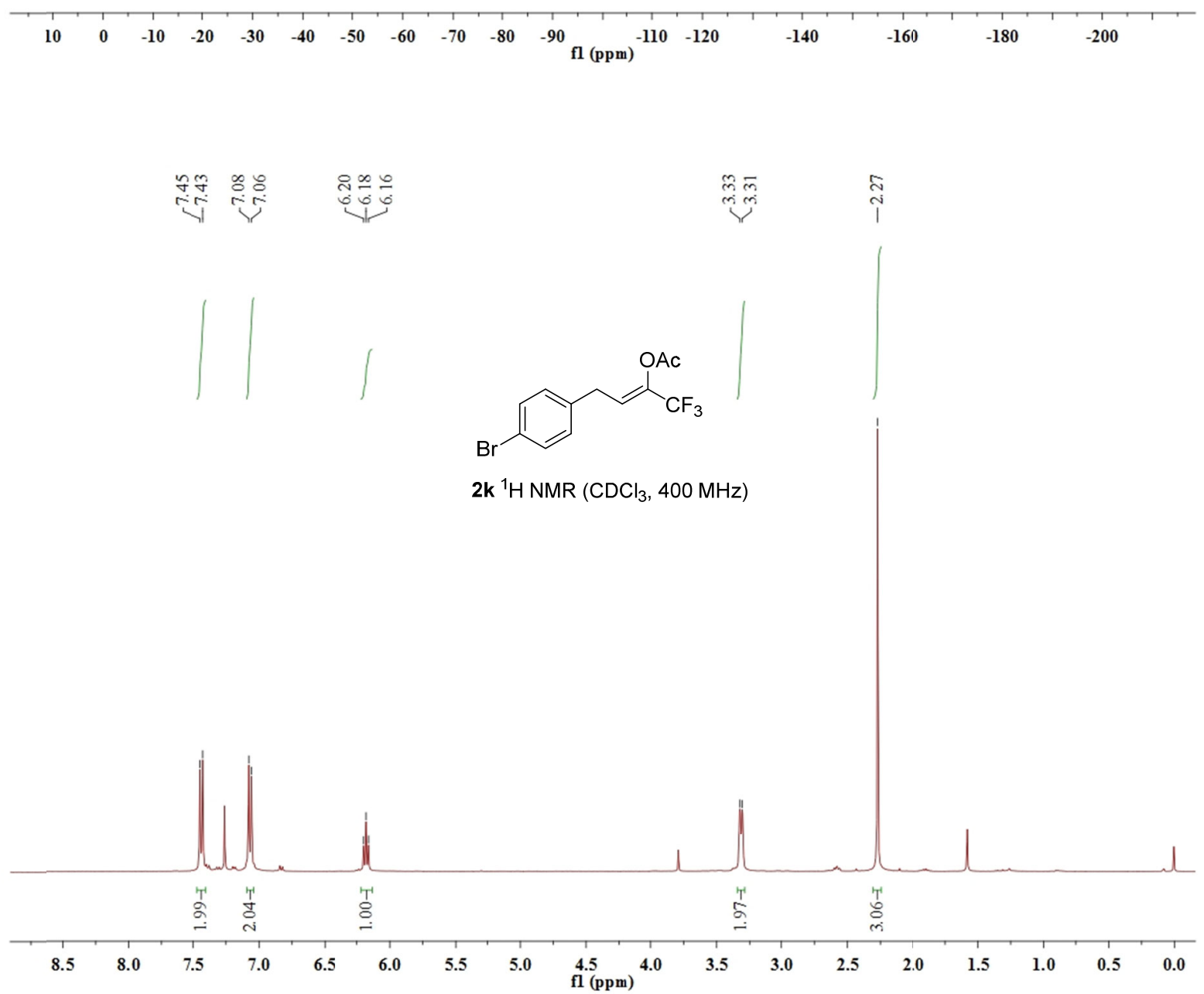




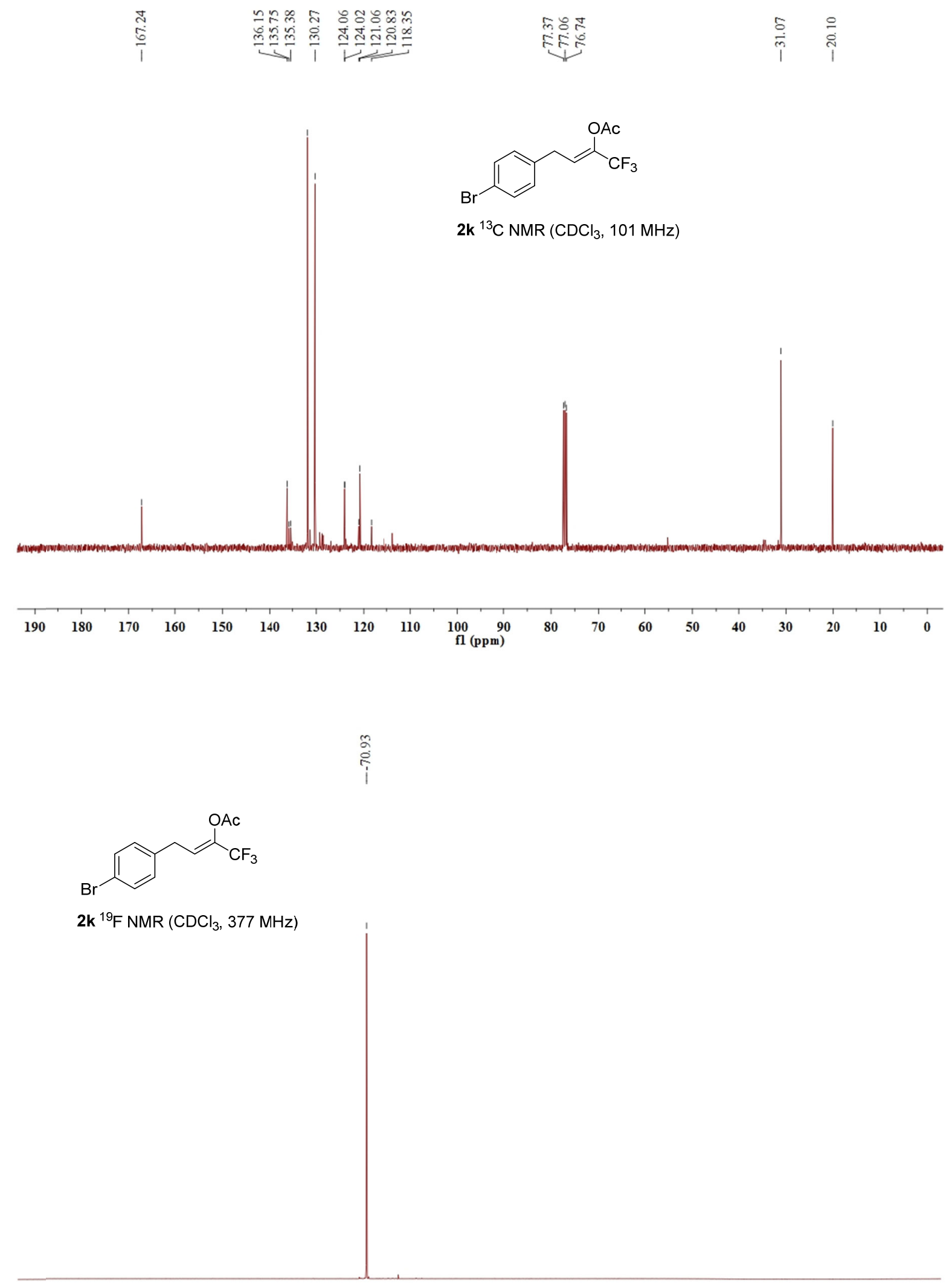

$\begin{array}{llllllllllllllllllll}1 & 0 & 0 & -10 & -20 & -30 & -40 & -50 & -60 & -70 & -80 & -90 & -110 & -120 & -140 & -160 & -180 & -200 & \end{array}$




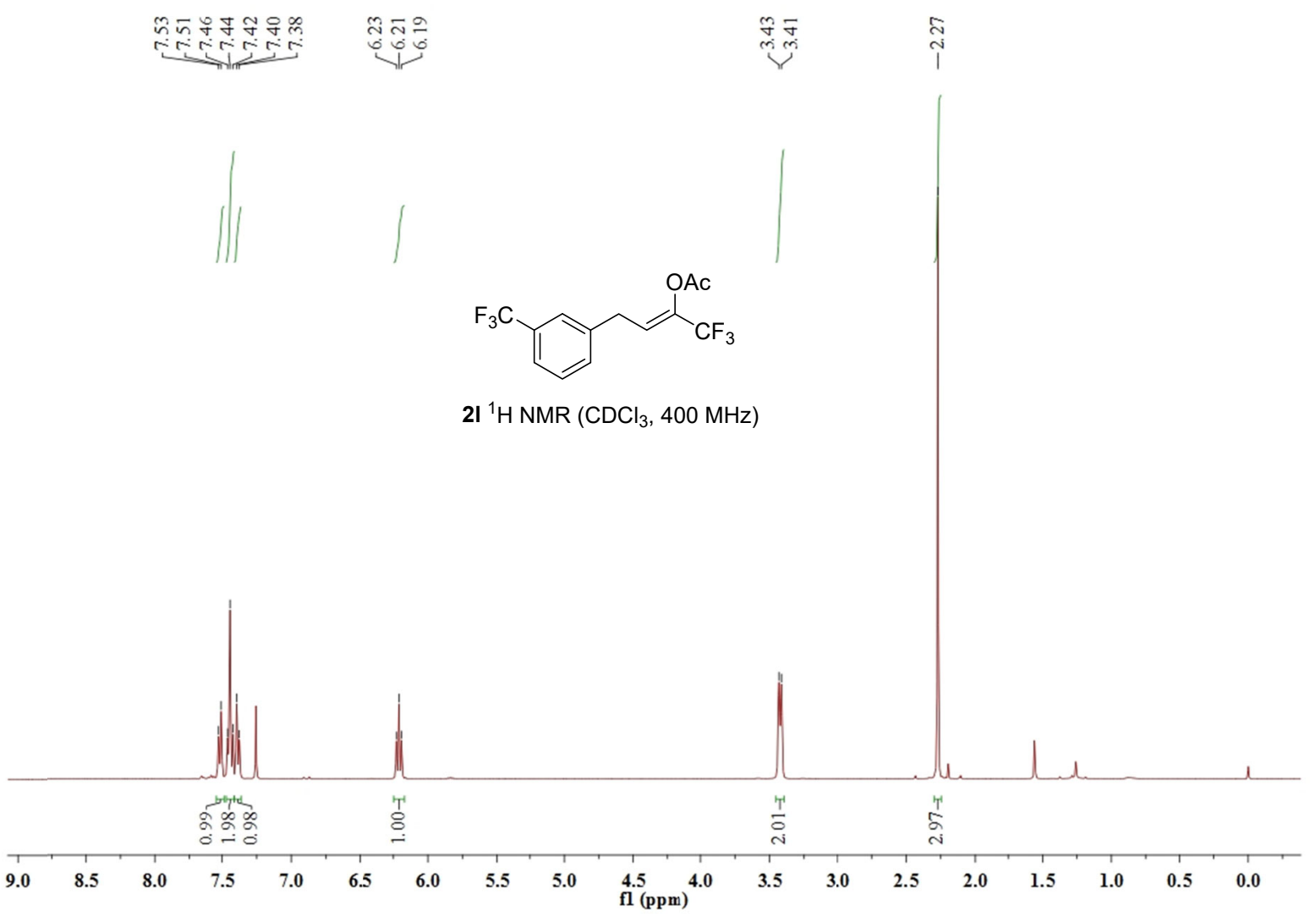

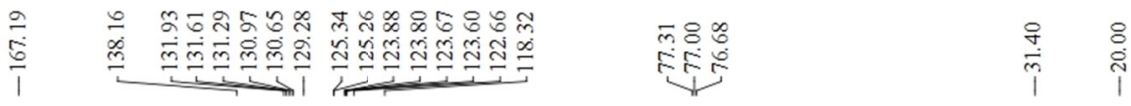

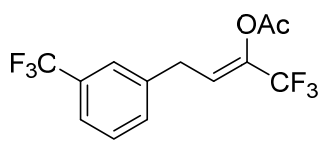

2l ${ }^{13} \mathrm{C}$ NMR $\left(\mathrm{CDCl}_{3}, 101 \mathrm{MHz}\right)$

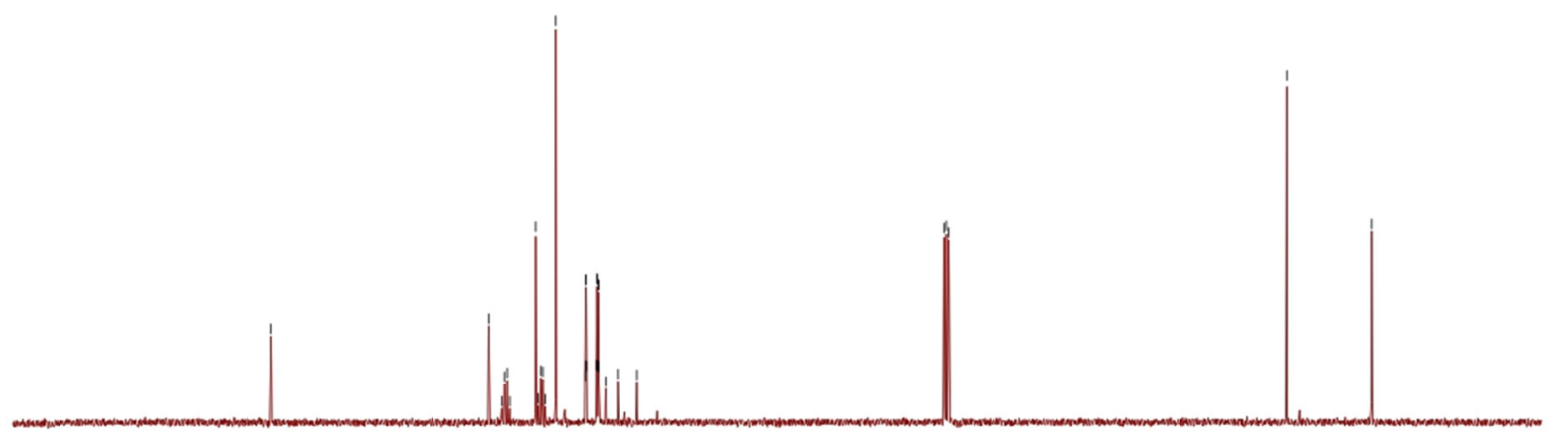

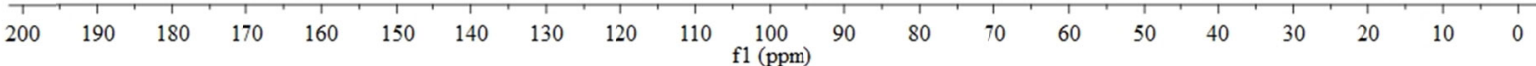




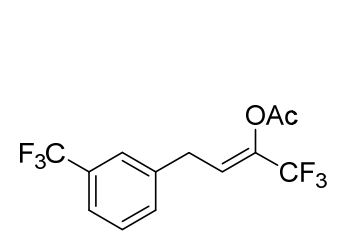

2I ${ }^{19} \mathrm{~F} \mathrm{NMR}\left(\mathrm{CDCl}_{3}, 377 \mathrm{MHz}\right)$

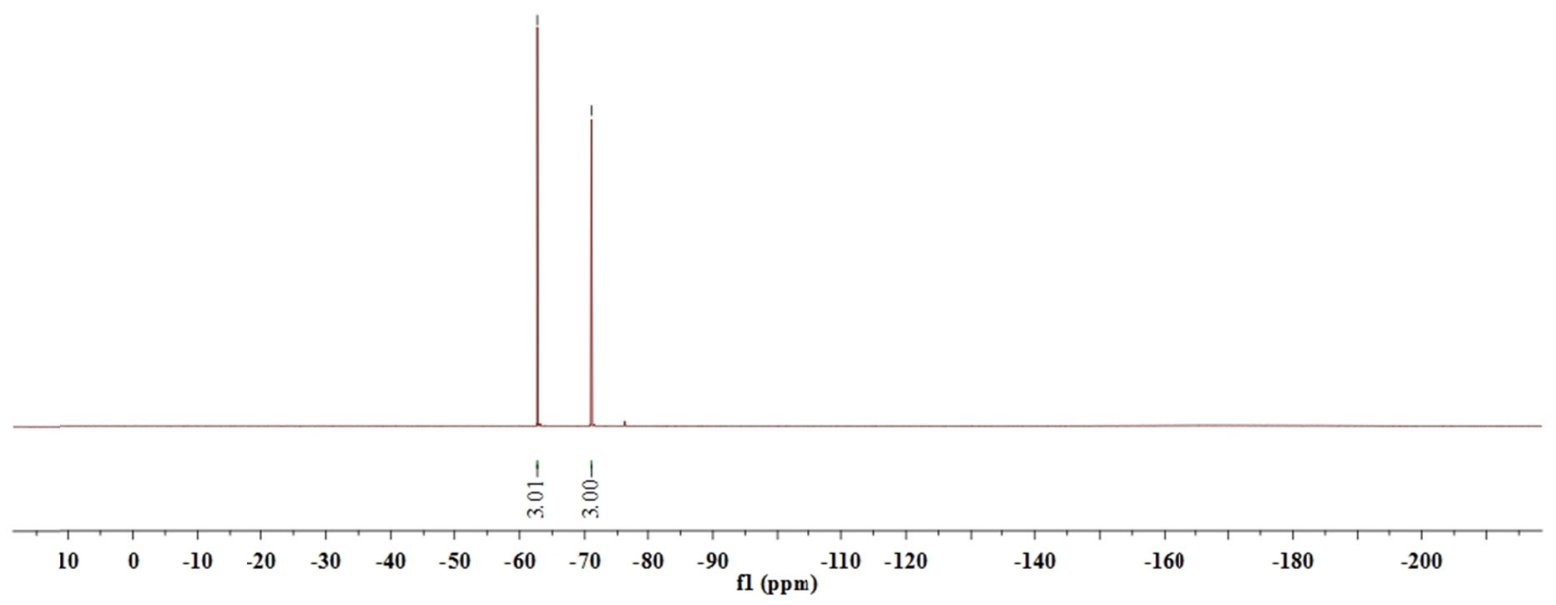

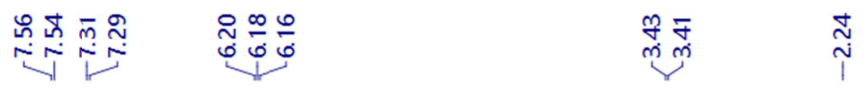

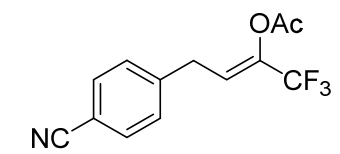

$2 \mathrm{~m}^{1} \mathrm{H} \mathrm{NMR}\left(\mathrm{CDCl}_{3}, 400 \mathrm{MHz}\right)$

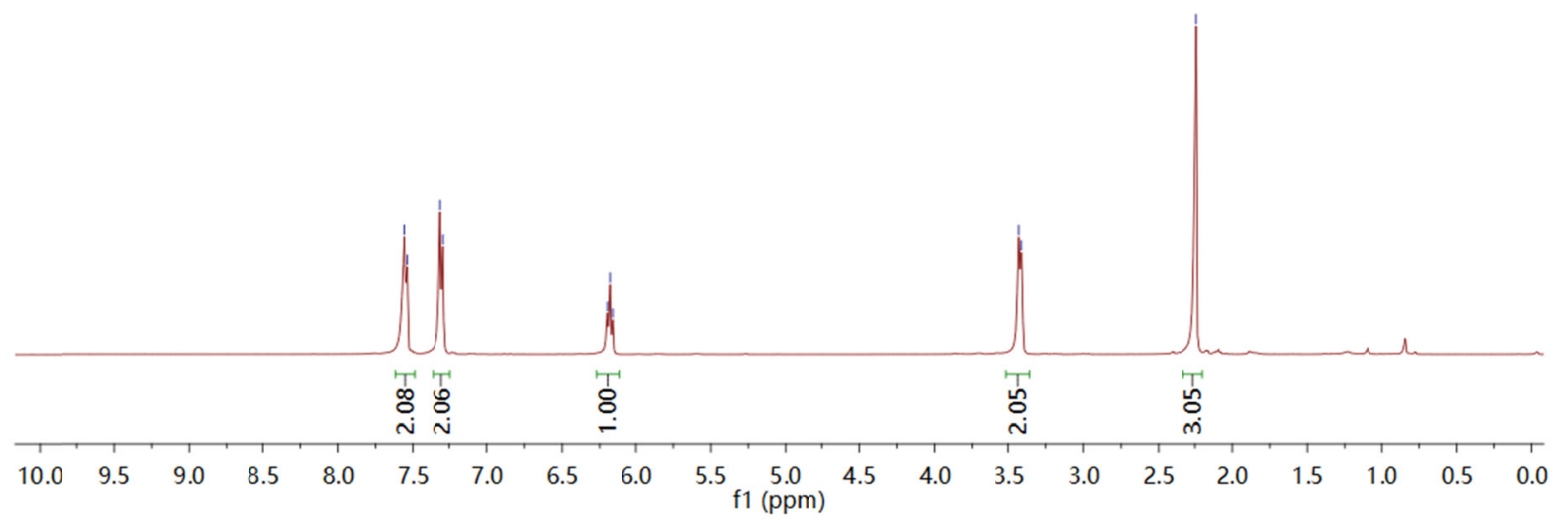



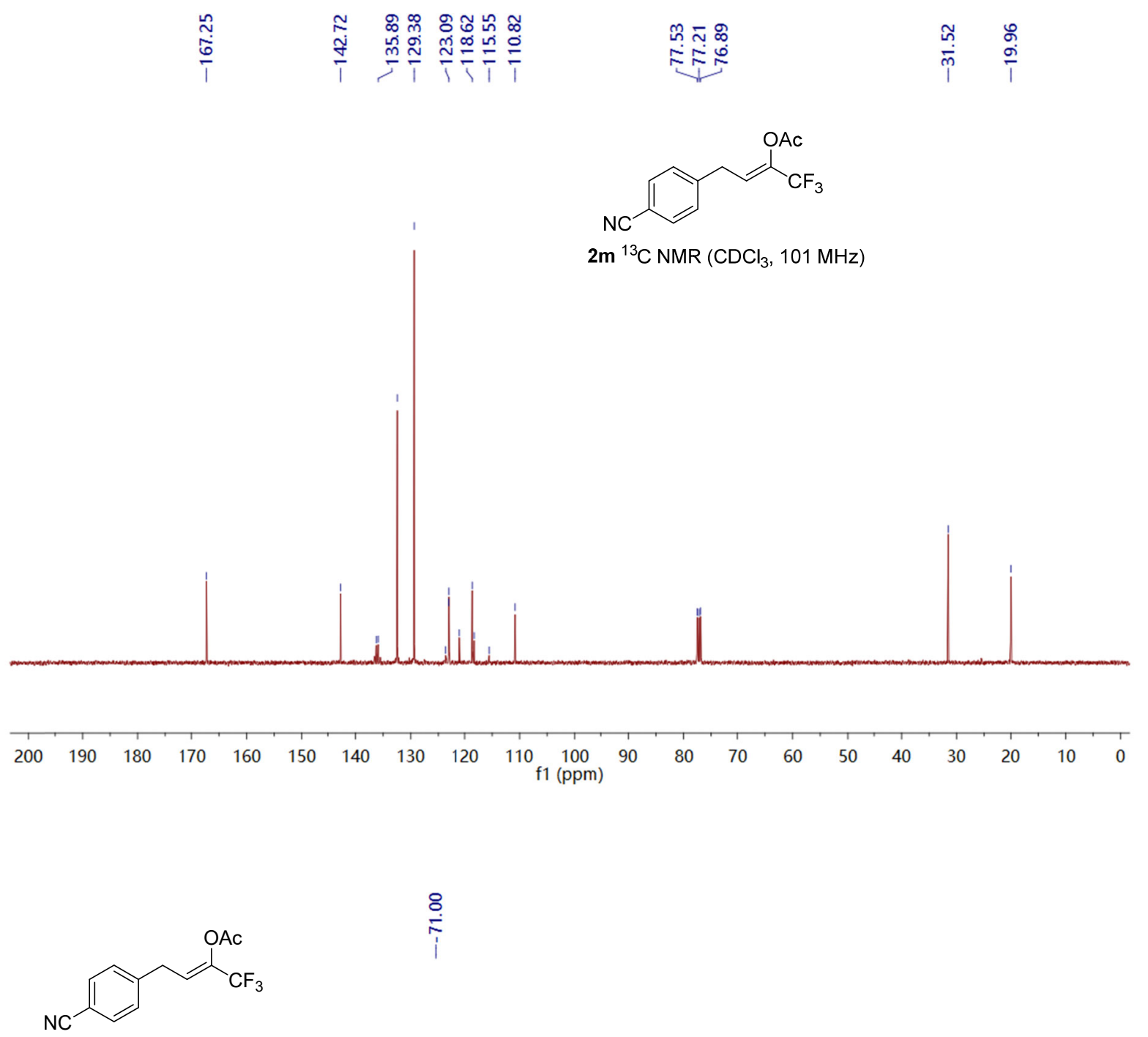

2m ${ }^{19} \mathrm{~F} \mathrm{NMR}\left(\mathrm{CDCl}_{3}, 377 \mathrm{MHz}\right)$

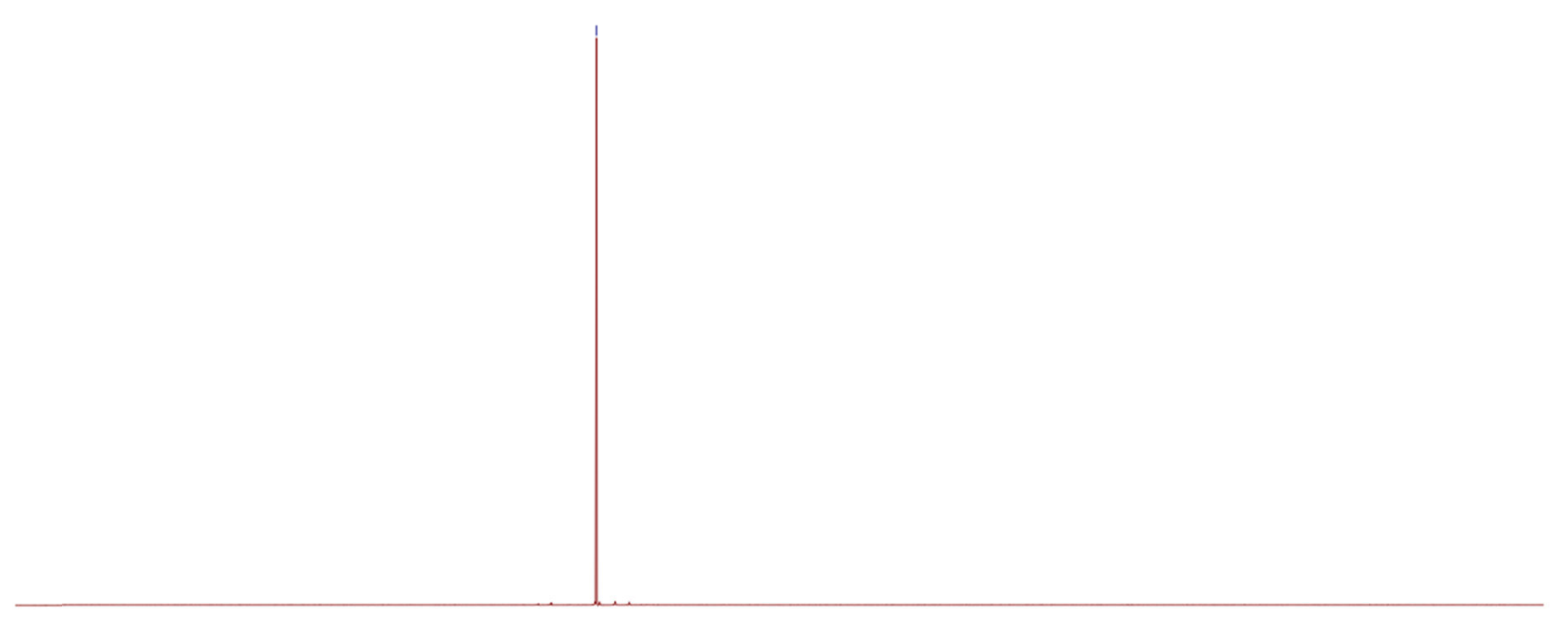

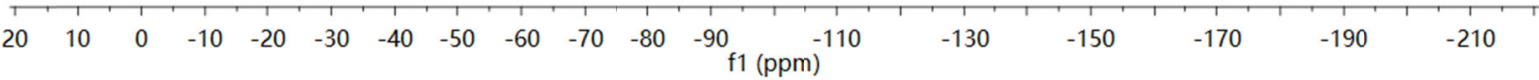




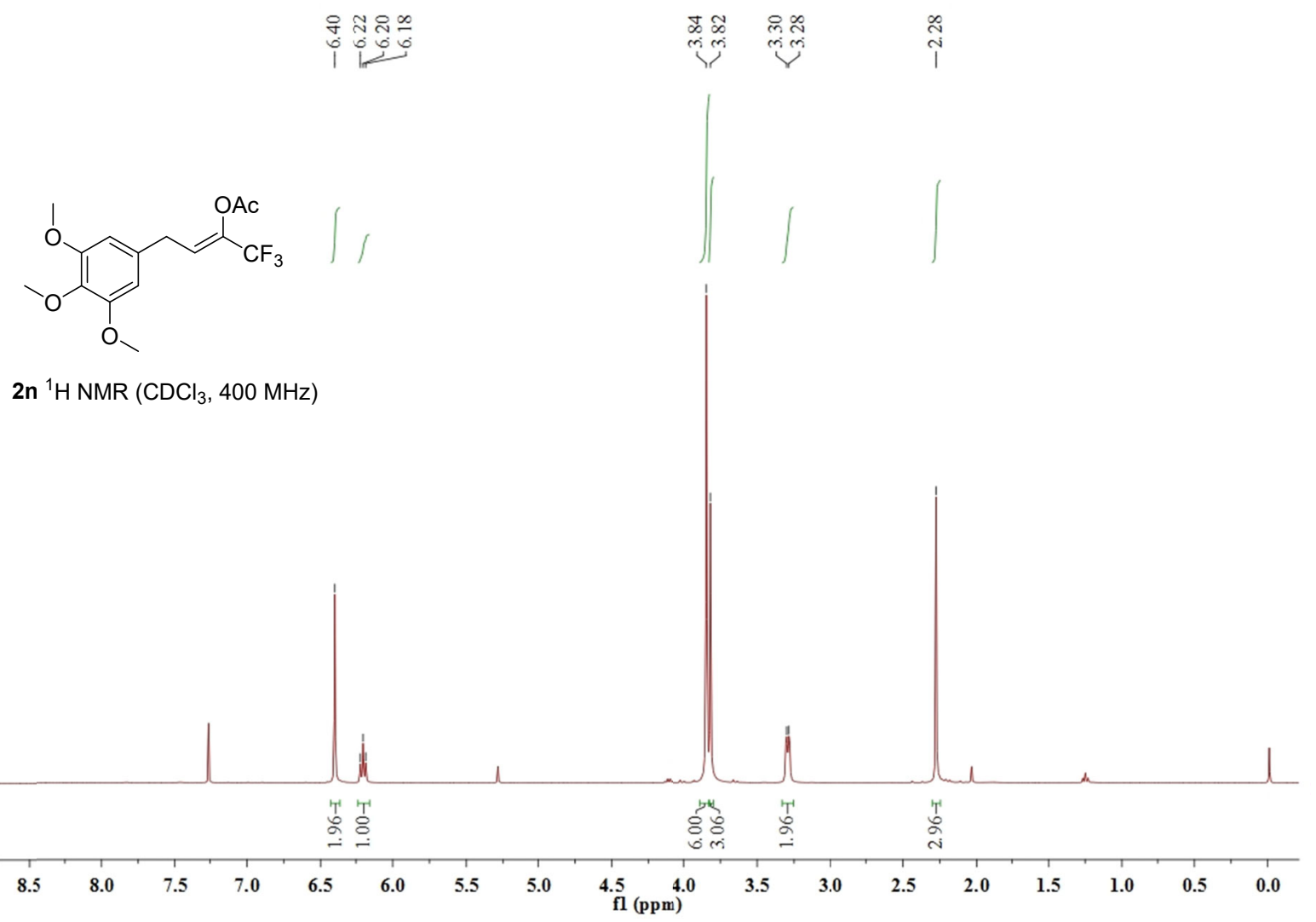

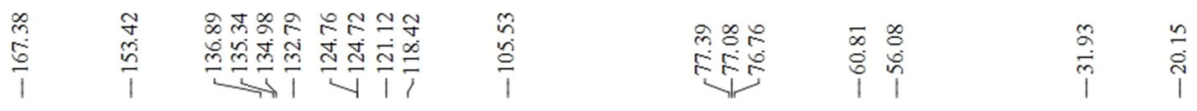<smiles>COc1cc(C/C=C(\OC(C)=O)C(F)(F)F)cc(OC)c1OC</smiles>

$2 n^{13} \mathrm{C}$ NMR $\left(\mathrm{CDCl}_{3}, 101 \mathrm{MHz}\right)$

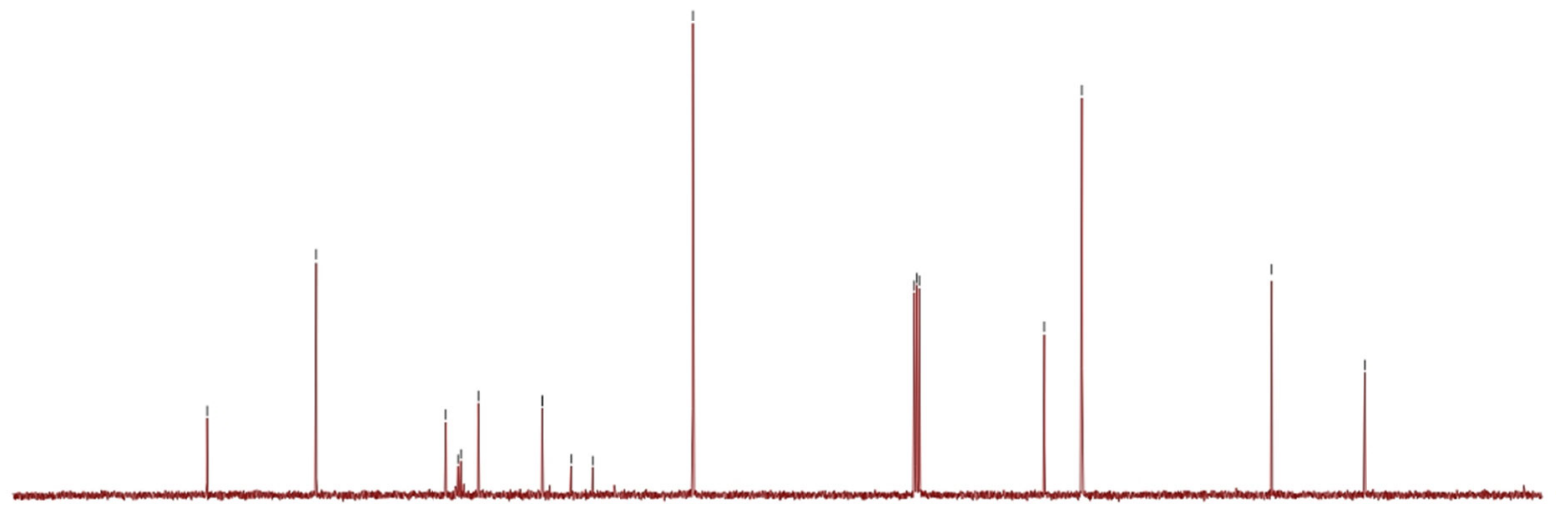

$\begin{array}{llllllllll}190 & 180 & 170 & 160 & 150 & 140 & 130 & 120 & 110 & \begin{array}{c}100 \\ \mathrm{fl}(\mathrm{ppm})\end{array}\end{array}$ 
<smiles>CO/C(=C\Cc1cc(OC)c(OC)c(OC)c1)C(F)(F)F</smiles>

2n ${ }^{19} \mathrm{~F} \mathrm{NMR}\left(\mathrm{CDCl}_{3}, 377 \mathrm{MHz}\right)$

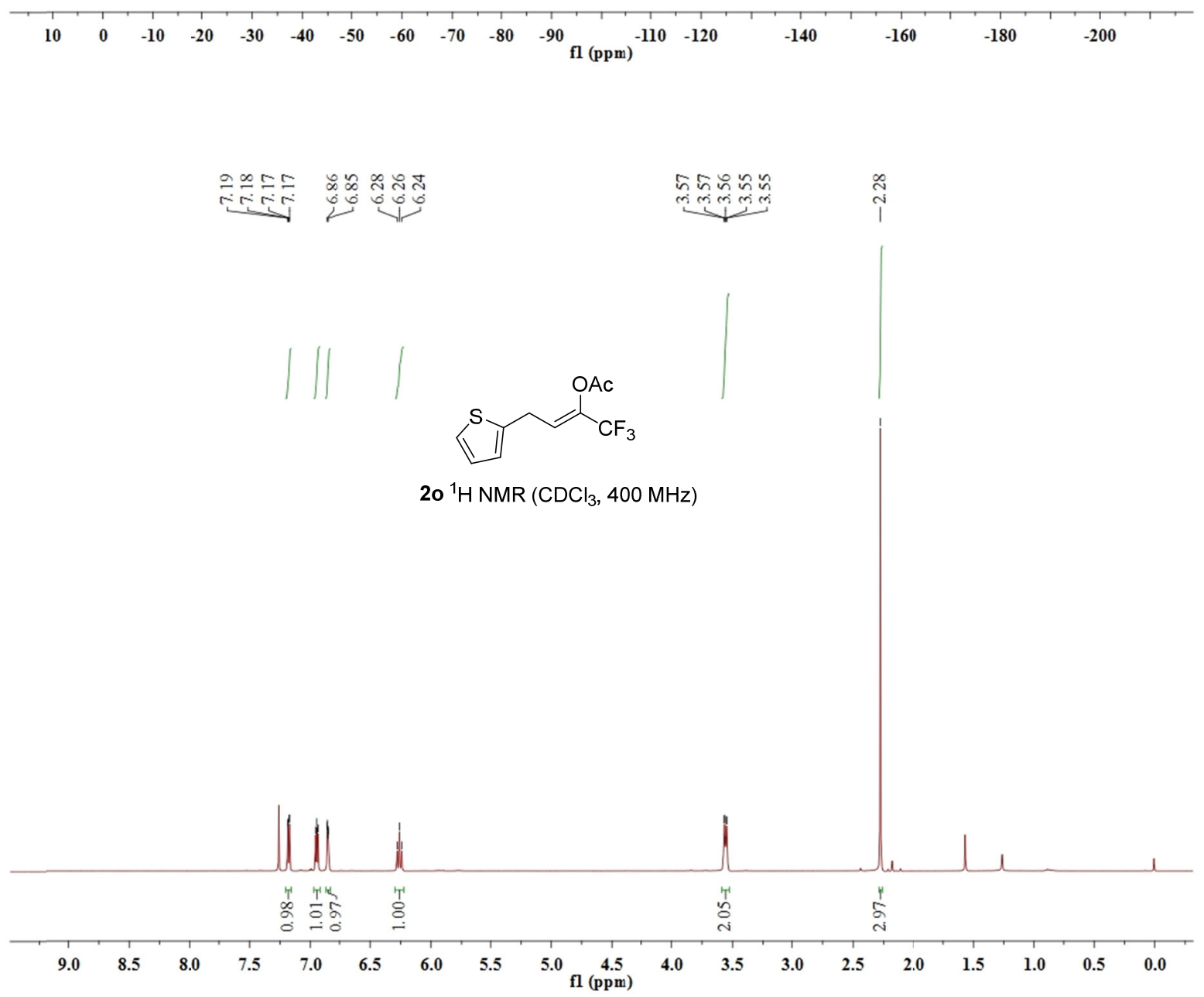




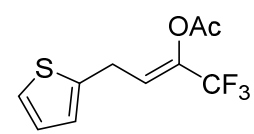

$20{ }^{13} \mathrm{C} \mathrm{NMR}\left(\mathrm{CDCl}_{3}, 101 \mathrm{MHz}\right)$

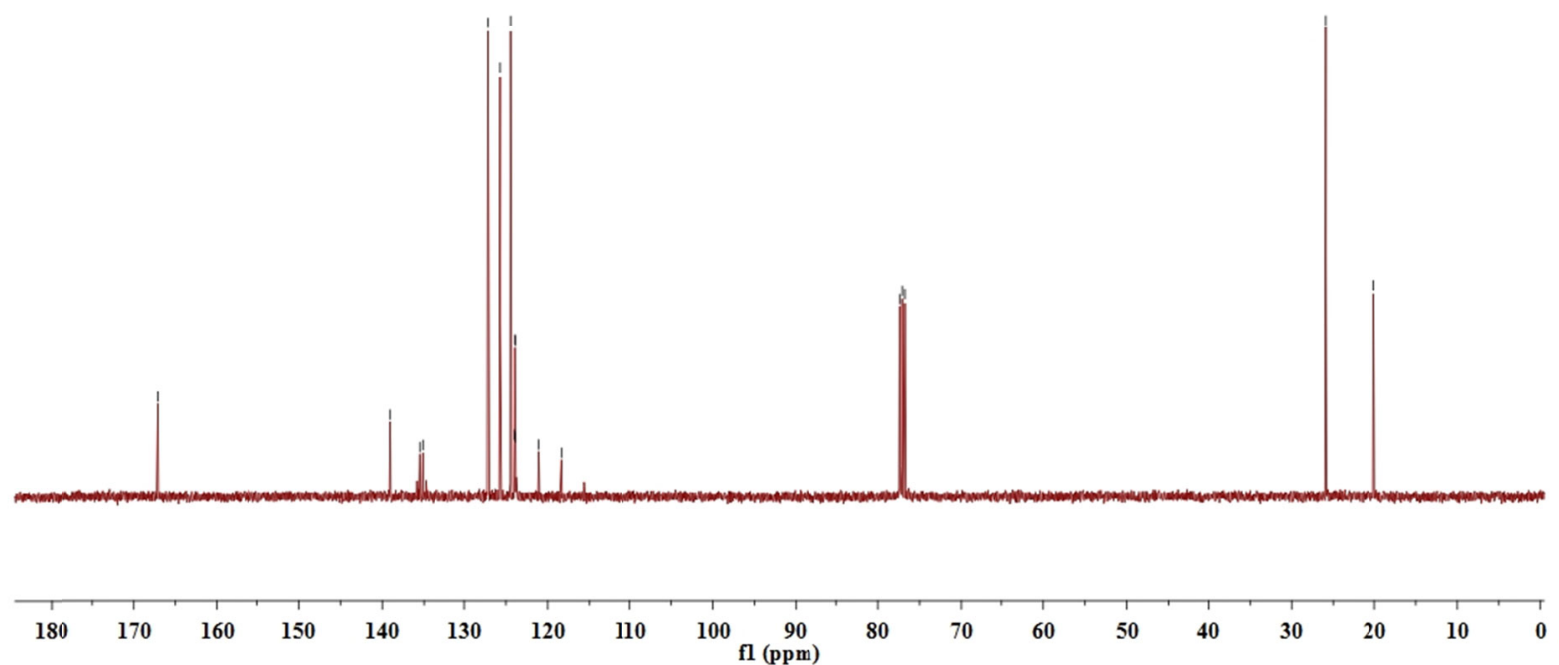<smiles>CC(=O)OC(=CCc1cccs1)C(F)(F)F</smiles>

20 ${ }^{19} \mathrm{~F} \mathrm{NMR}\left(\mathrm{CDCl}_{3}, 377 \mathrm{MHz}\right)$

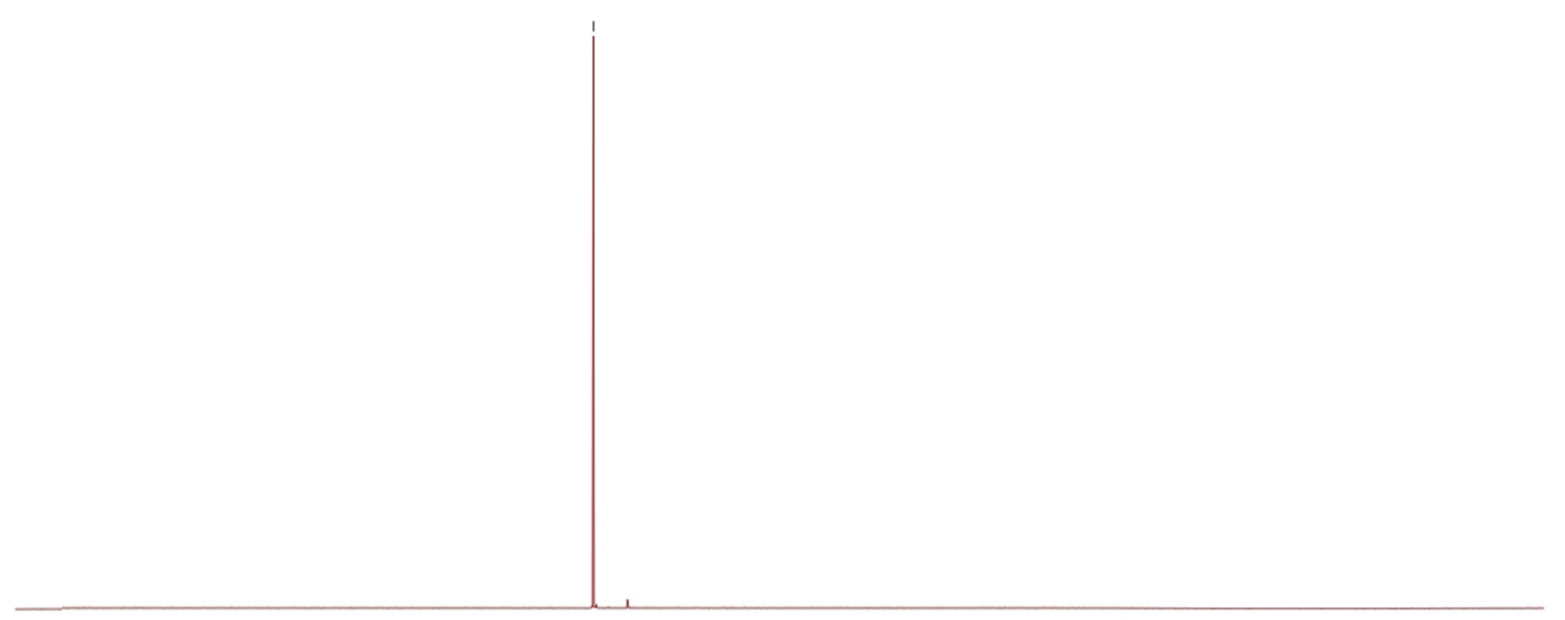




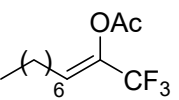

2p ${ }^{1} \mathrm{H} \mathrm{NMR}\left(\mathrm{CDCl}_{3}, 400 \mathrm{MHz}\right)$
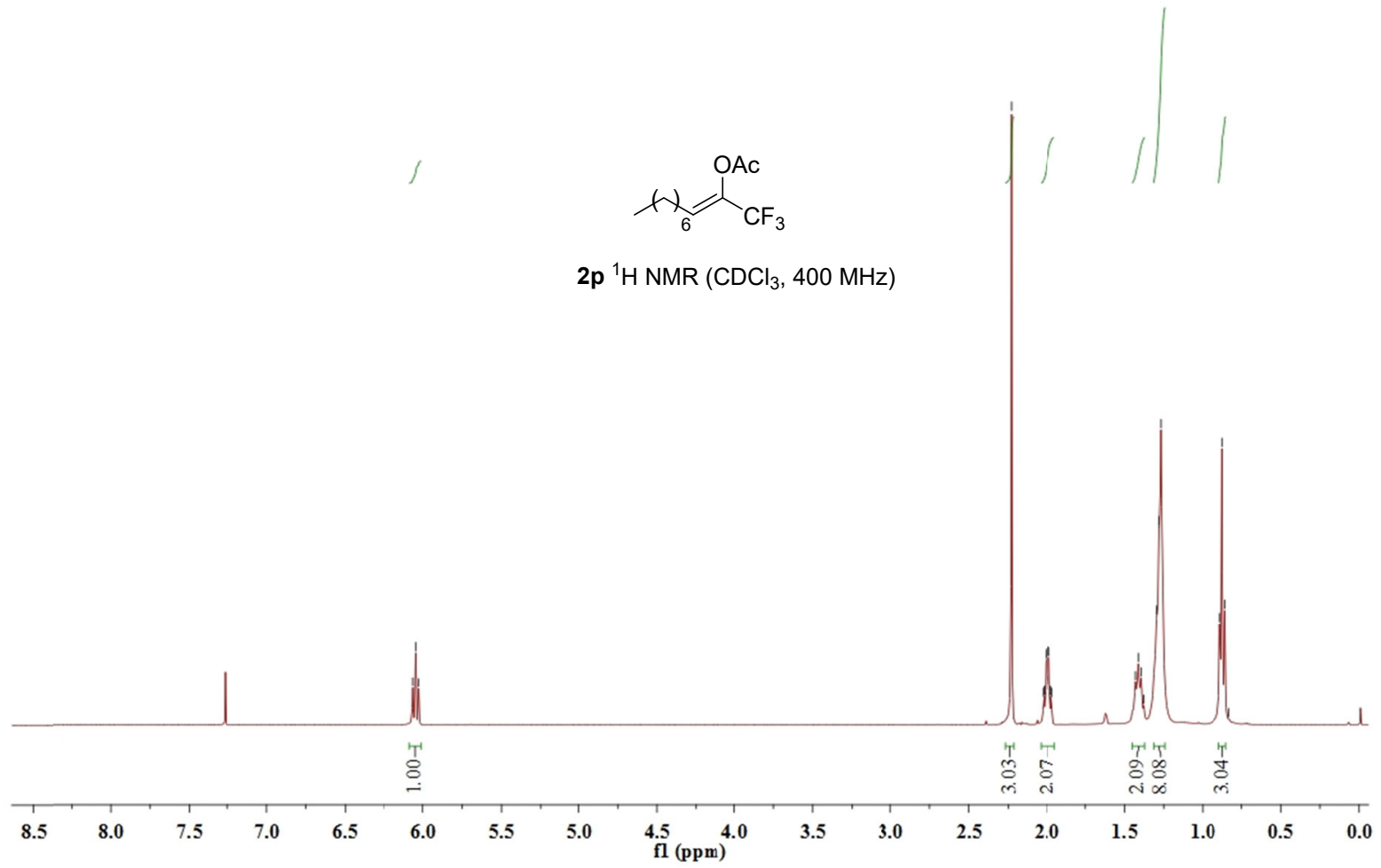

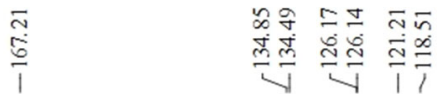

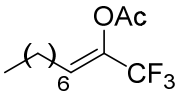

$2 p{ }^{13} \mathrm{C} \mathrm{NMR}\left(\mathrm{CDCl}_{3}, 101 \mathrm{MHz}\right)$

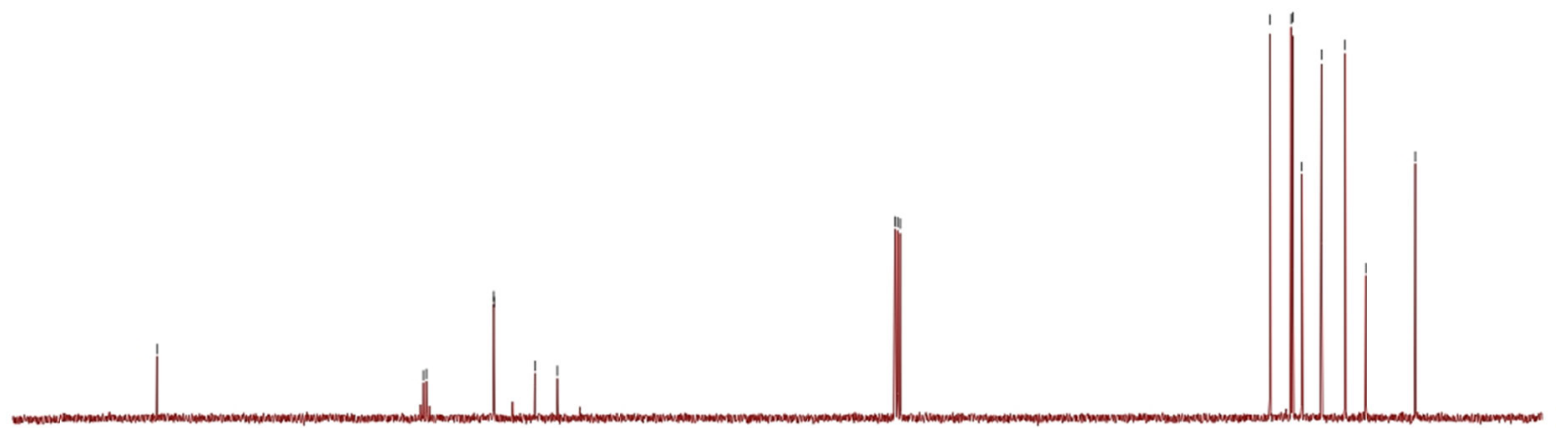

$\begin{array}{llllllll}180 & 170 & 160 & 150 & 140 & 130 & 120 & 110\end{array}$ $100 \underset{\text { fl }(\mathrm{ppm})}{90}$

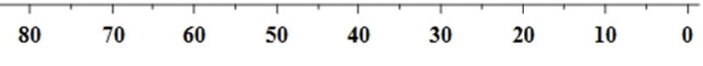


<smiles>CC(C)=CC(=CC(C)C)C(F)(F)F</smiles>

2p ${ }^{19} \mathrm{~F} \mathrm{NMR}\left(\mathrm{CDCl}_{3}, 377 \mathrm{MHz}\right)$

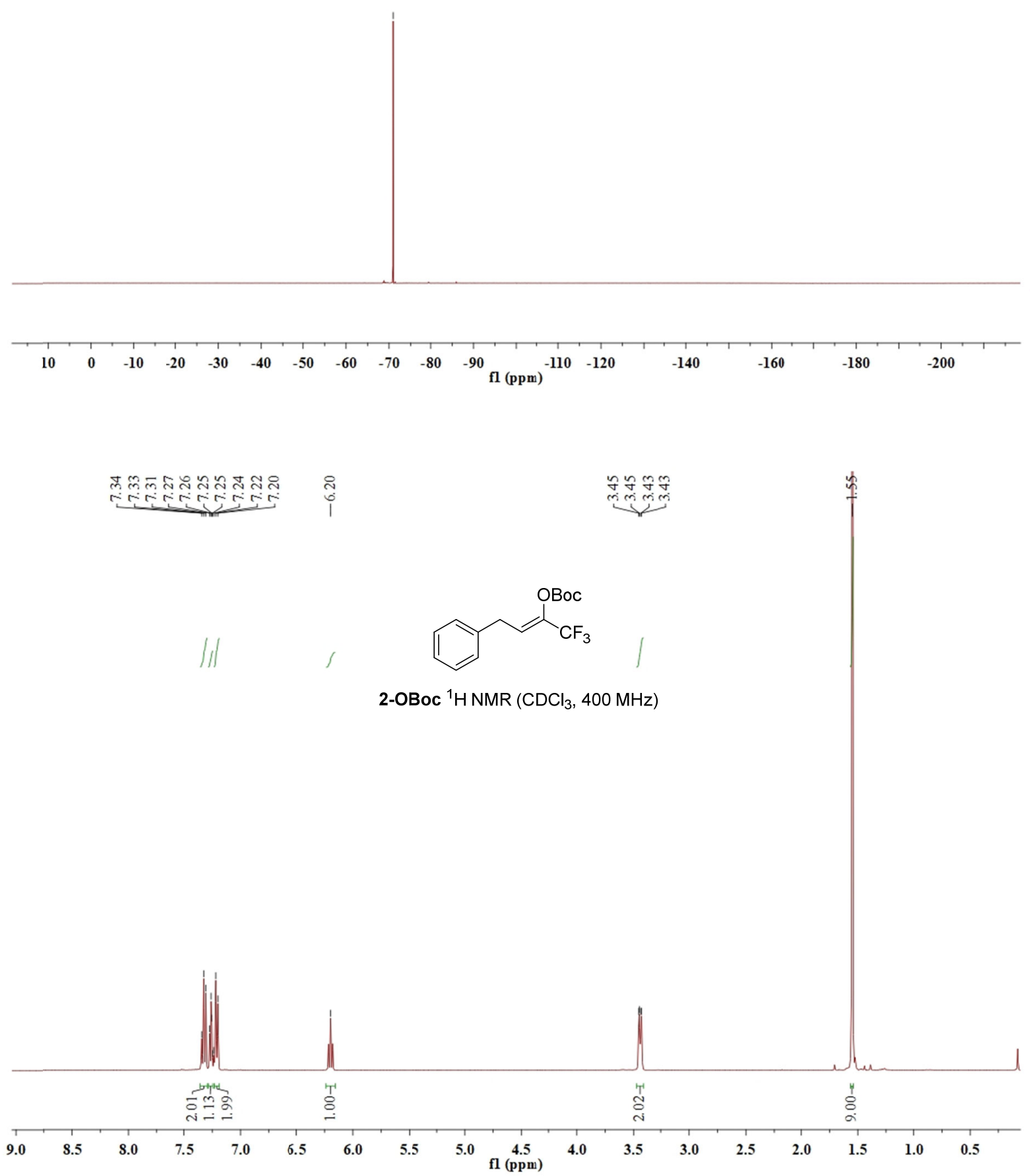




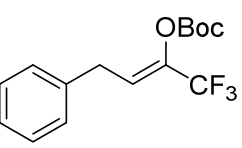

2-OBoc $\left.{ }^{13} \mathrm{C} \mathrm{NMR} \mathrm{(} \mathrm{CDCl}_{3}, 101 \mathrm{MHz}\right)$

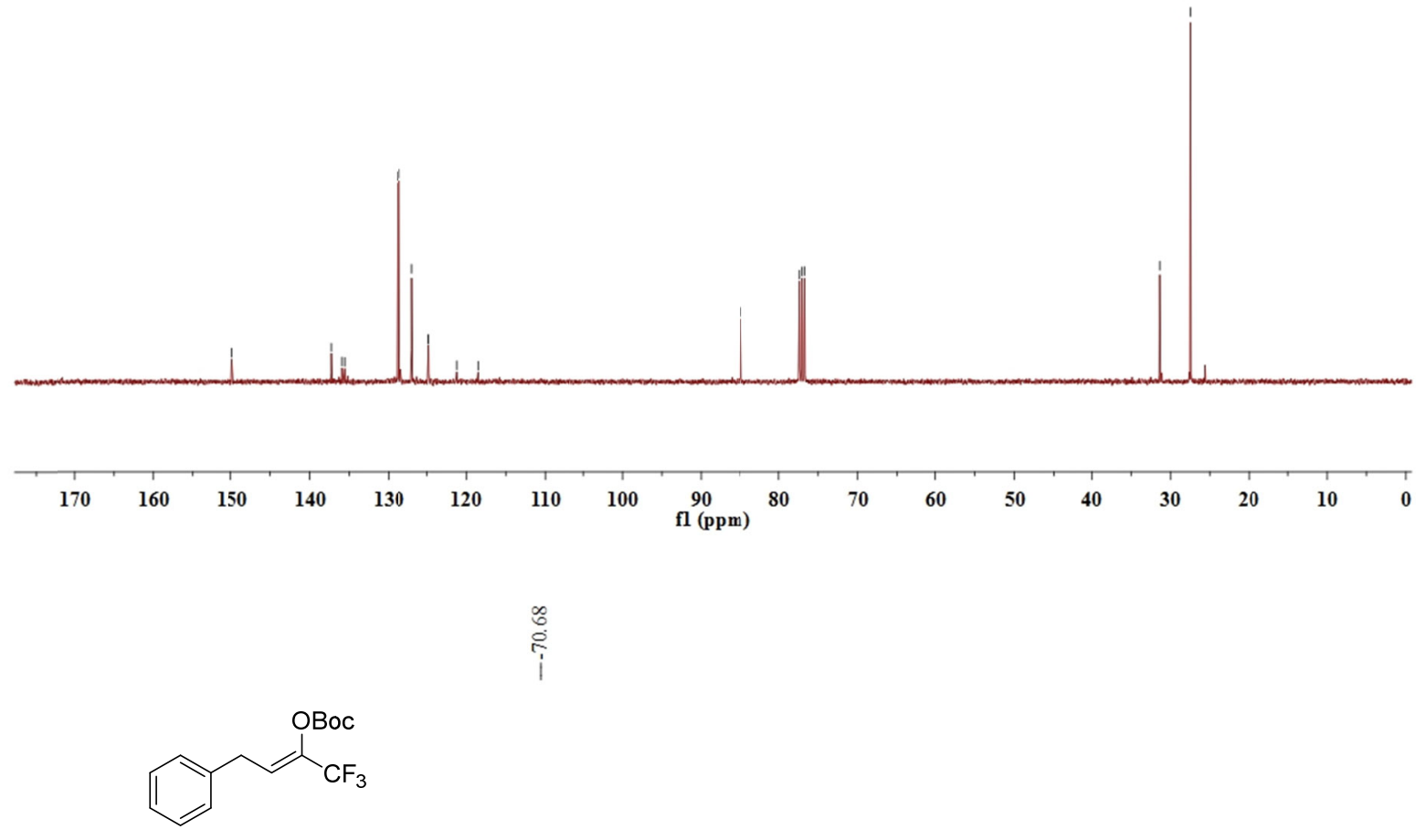

2-OBoc ${ }^{19} \mathrm{~F} \mathrm{NMR}\left(\mathrm{CDCl}_{3}, 377 \mathrm{MHz}\right)$

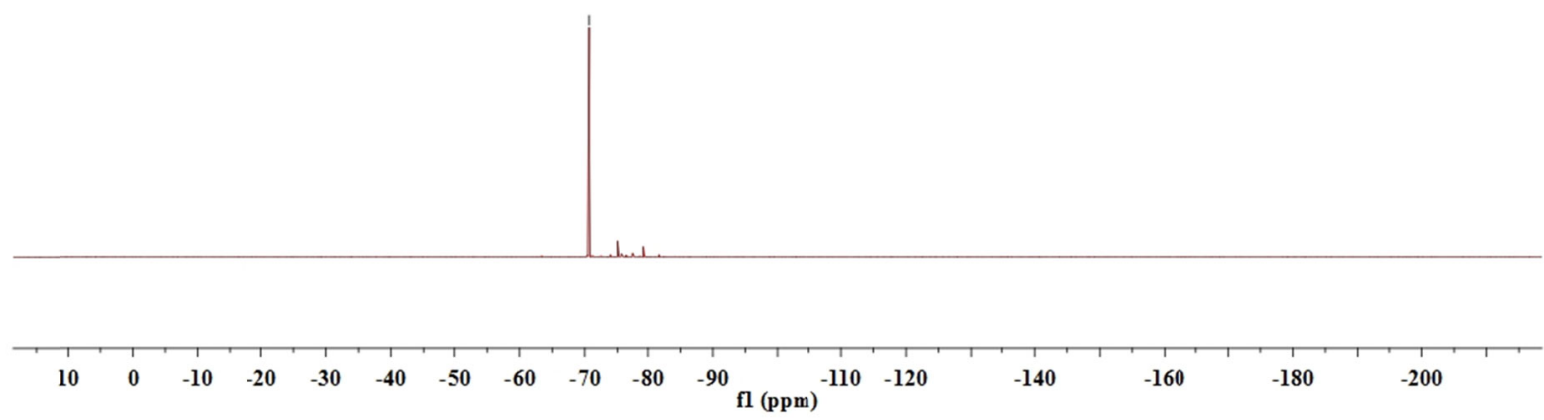




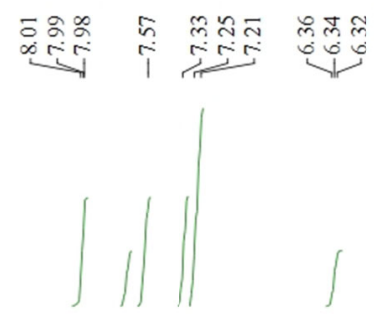

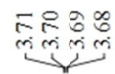
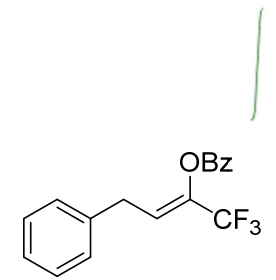

2-OBz ${ }^{1} \mathrm{H} \mathrm{NMR}\left(\mathrm{CDCl}_{3}, 400 \mathrm{MHz}\right)$

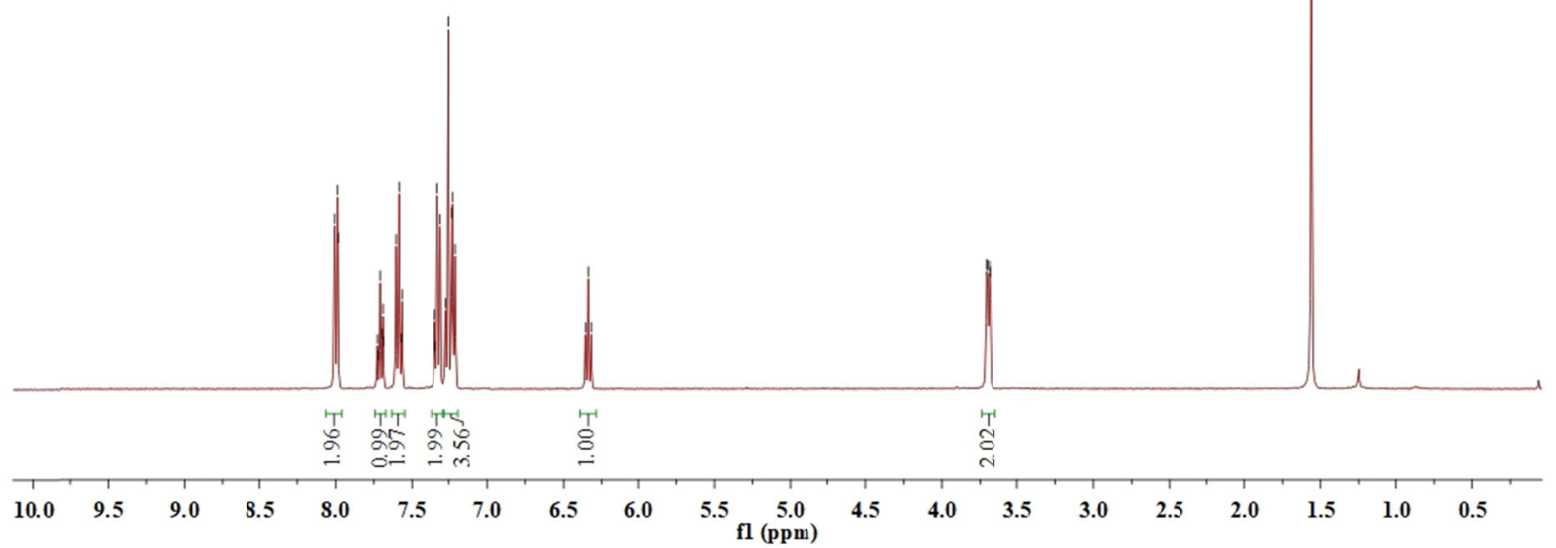

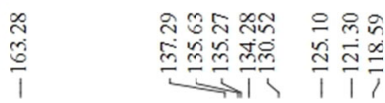

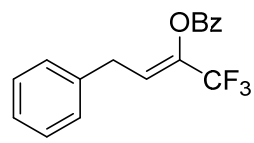

2-OBz ${ }^{13} \mathrm{C} \mathrm{NMR}\left(\mathrm{CDCl}_{3}, 101 \mathrm{MHz}\right)$

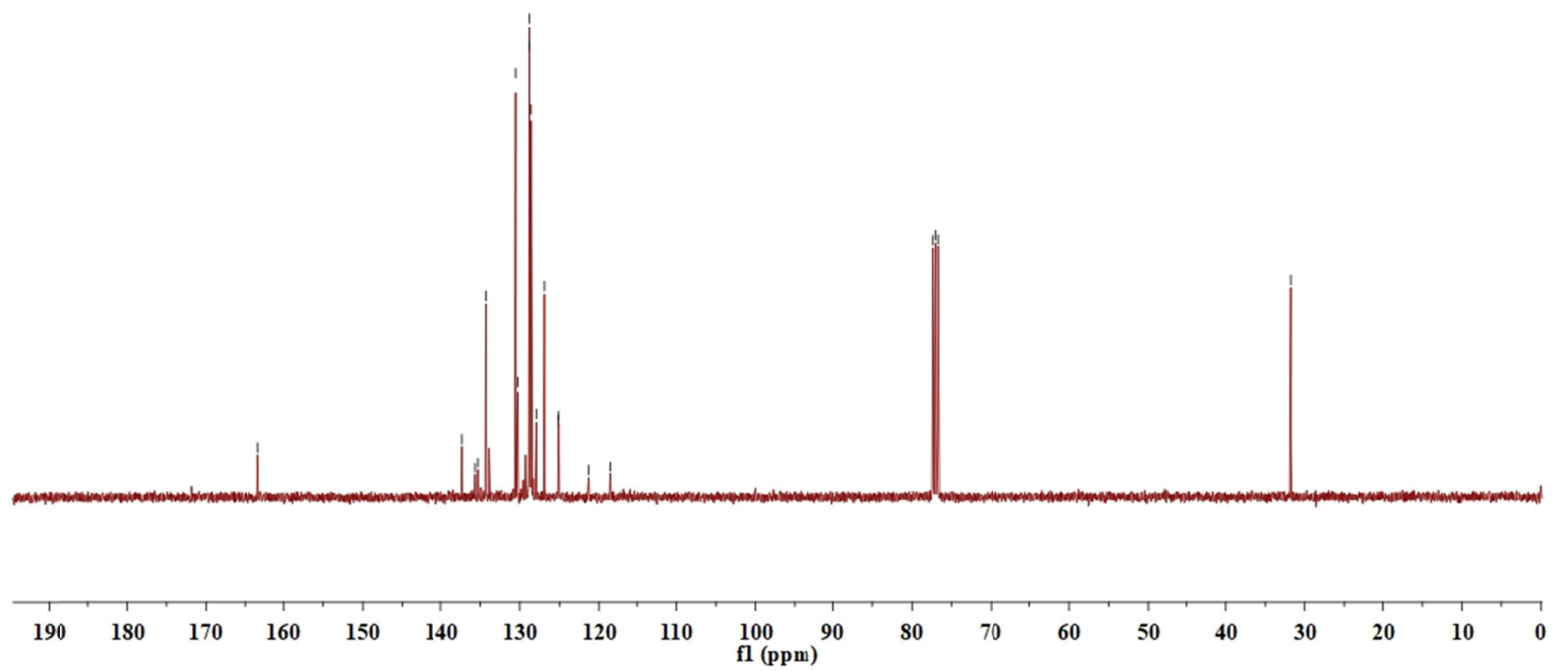


<smiles>CC(C)(C)OC(=CCc1ccccc1)C(F)(F)F</smiles>

2-OBz ${ }^{19} \mathrm{~F} \mathrm{NMR}\left(\mathrm{CDCl}_{3}, 377 \mathrm{MHz}\right)$

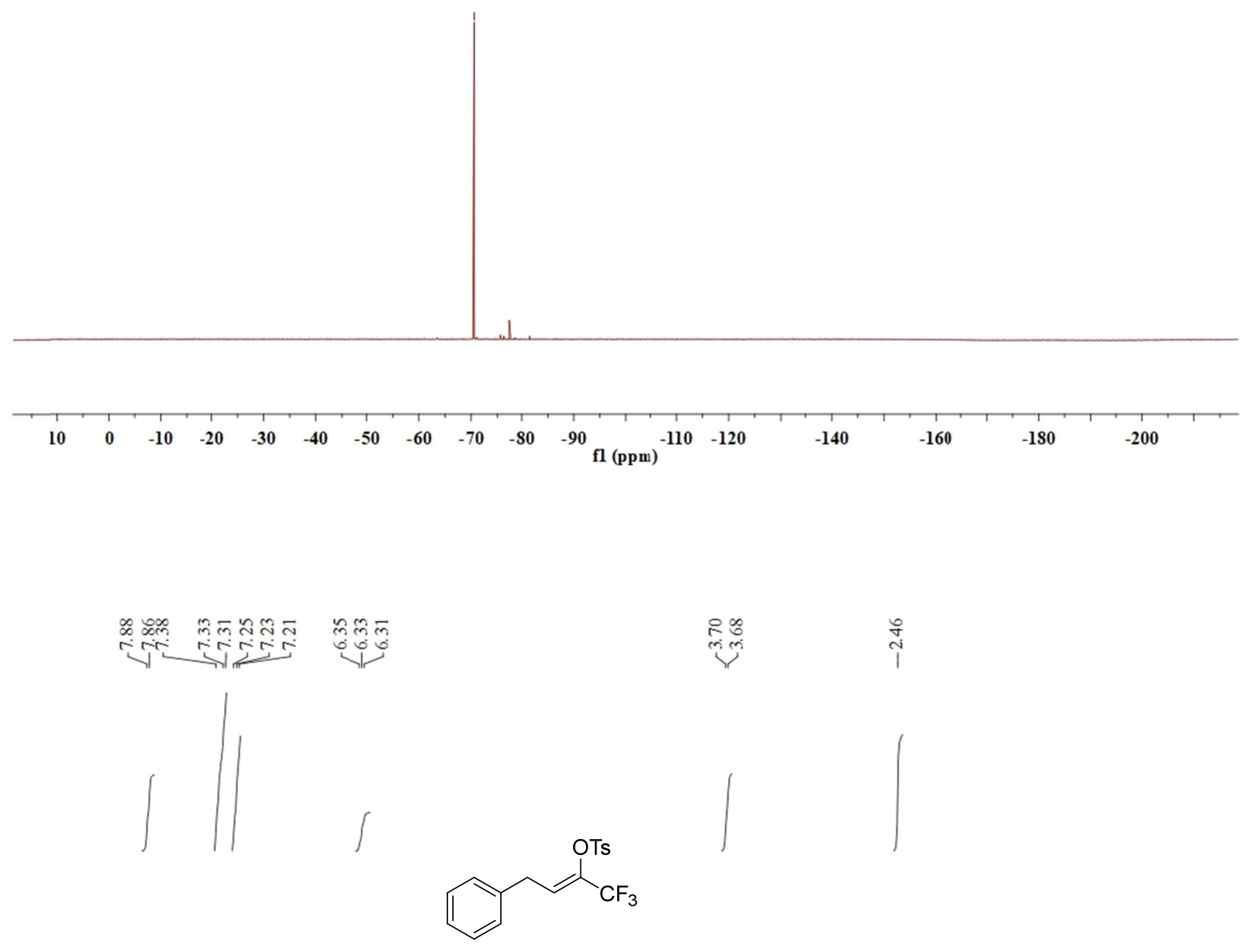

2-OTs ${ }^{1} \mathrm{H}$ NMR $\left(\mathrm{CDCl}_{3}, 400 \mathrm{MHz}\right)$

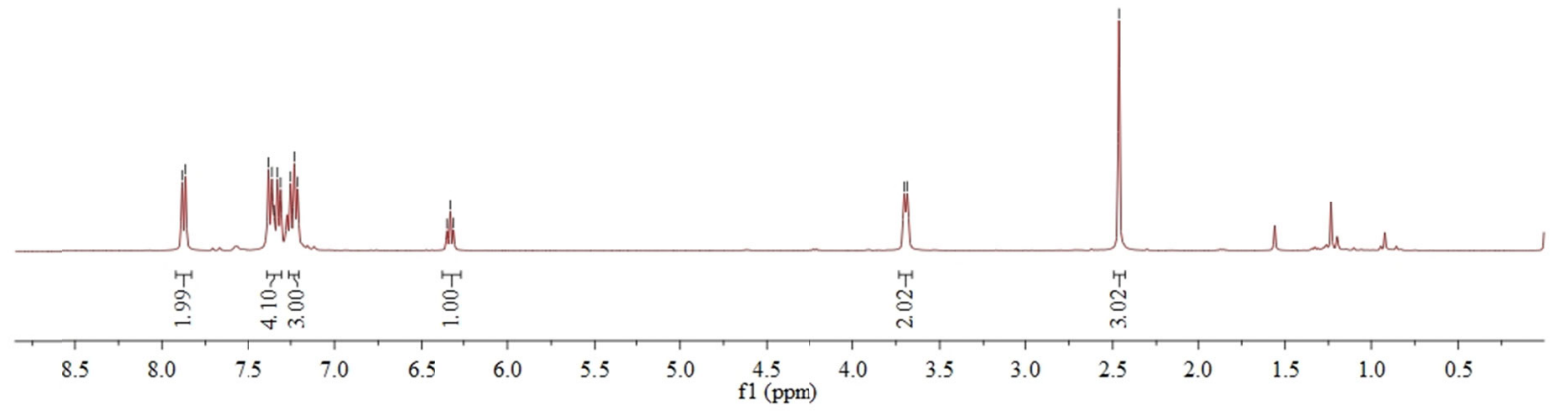




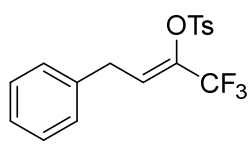

2-OTs ${ }^{13} \mathrm{C} \mathrm{NMR}\left(\mathrm{CDCl}_{3}, 101 \mathrm{MHz}\right)$
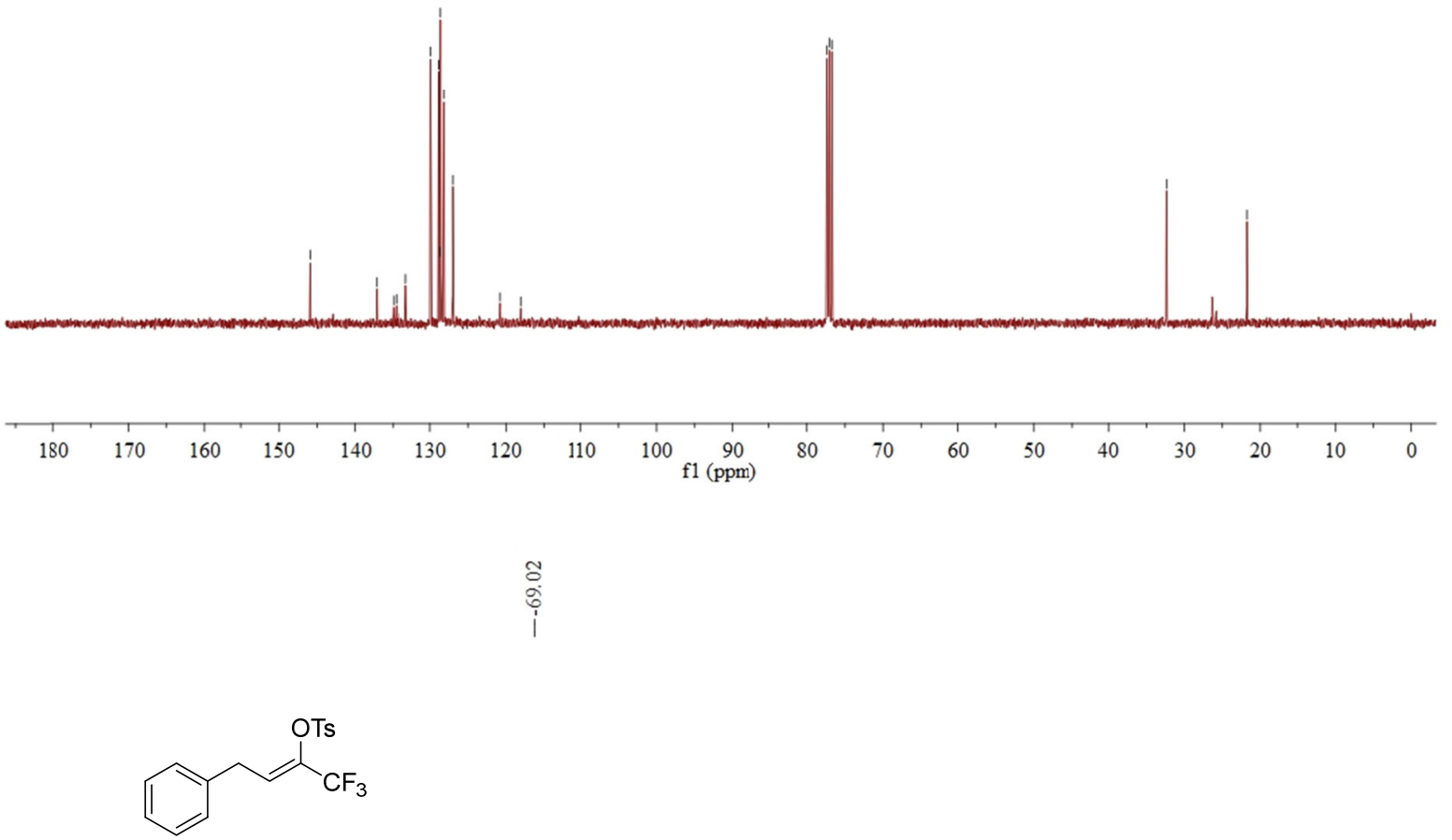

2-OTs ${ }^{19} \mathrm{~F} \mathrm{NMR}\left(\mathrm{CDCl}_{3}, 377 \mathrm{MHz}\right)$

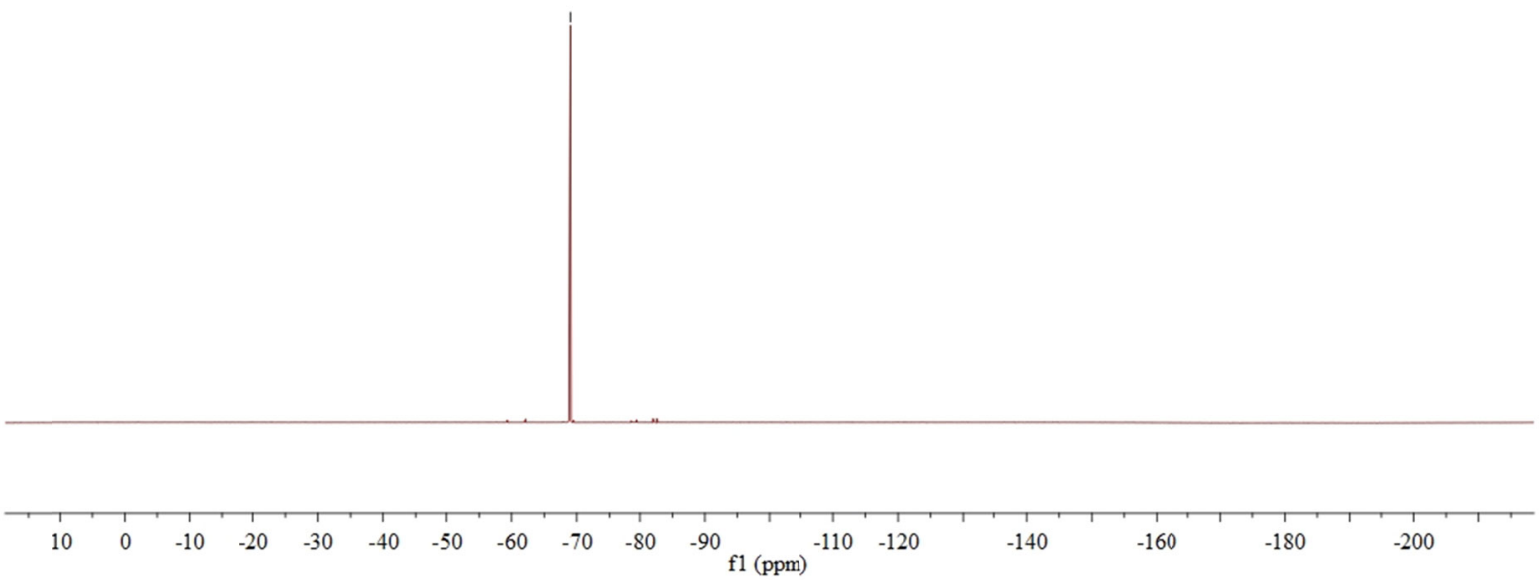




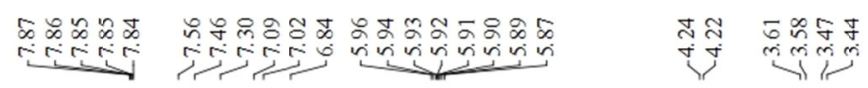
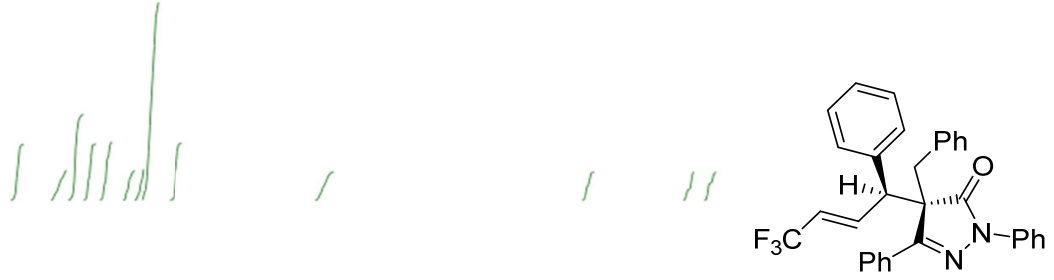

4a ${ }^{1} \mathrm{H}$ NMR $\left(\mathrm{CDCl}_{3}, 400 \mathrm{MHz}\right)$

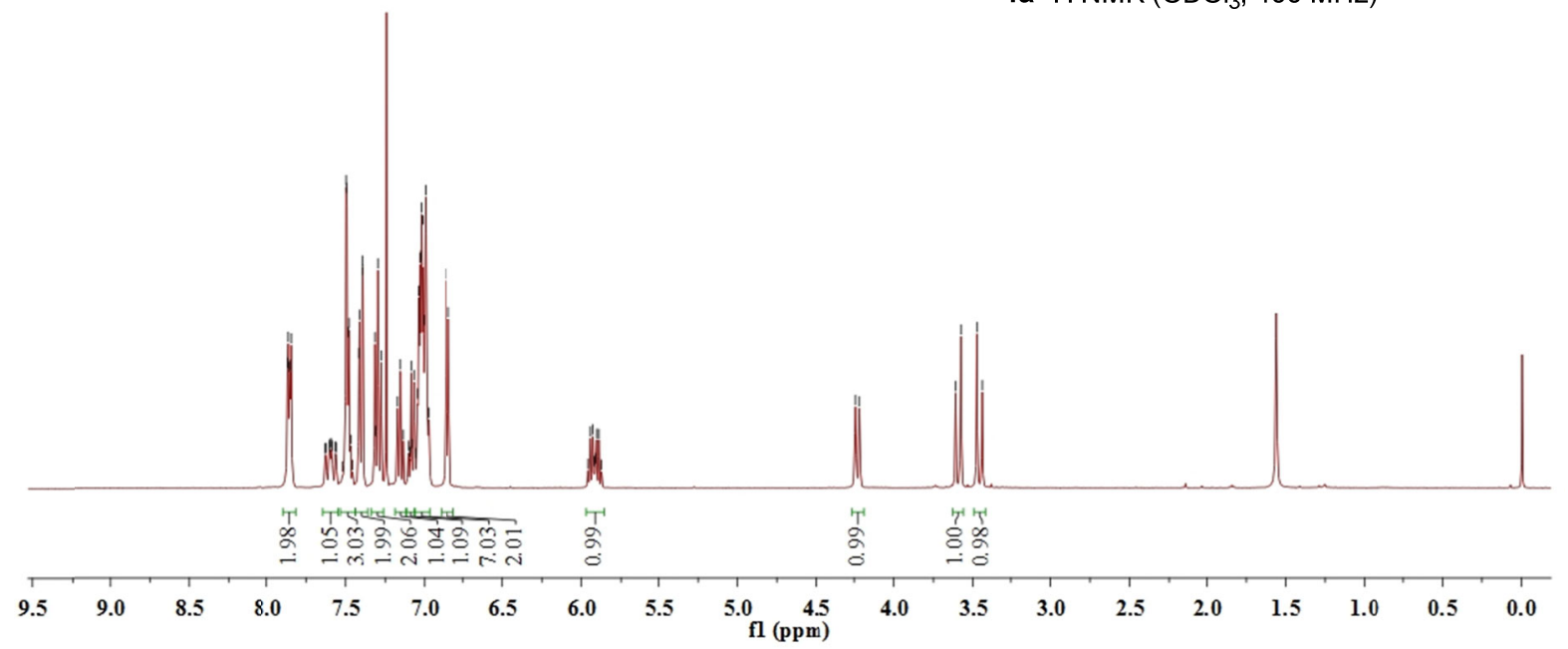

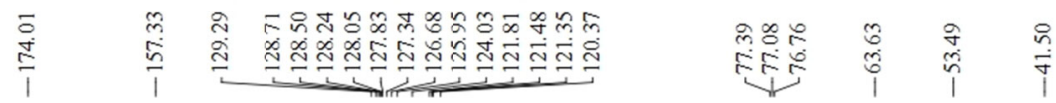

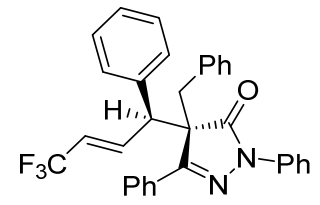

4a ${ }^{13} \mathrm{C} \mathrm{NMR}\left(\mathrm{CDCl}_{3}, 101 \mathrm{MHz}\right)$

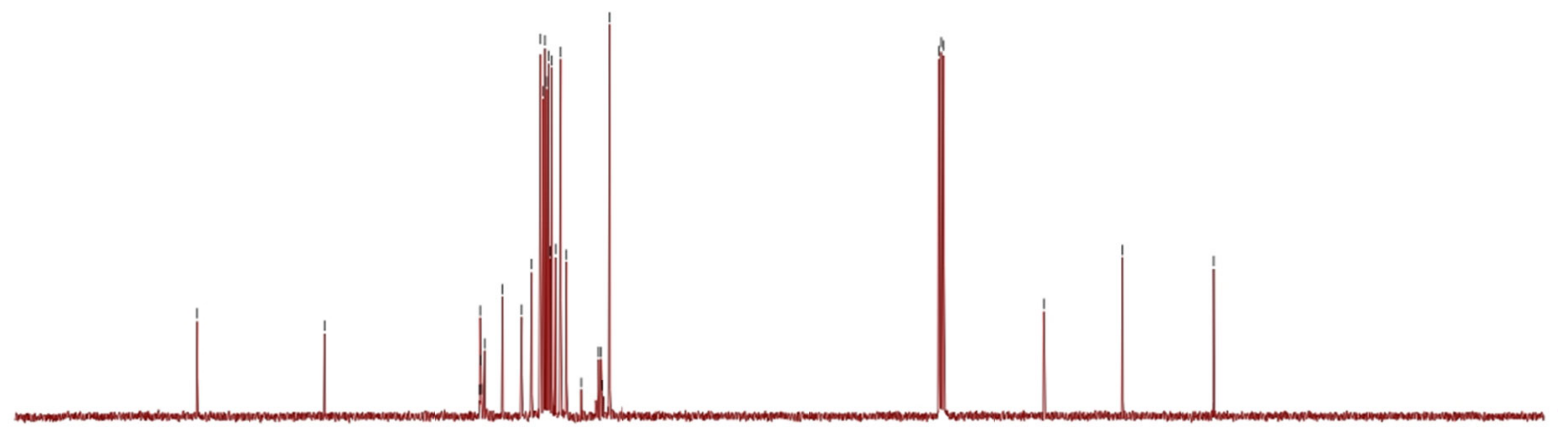

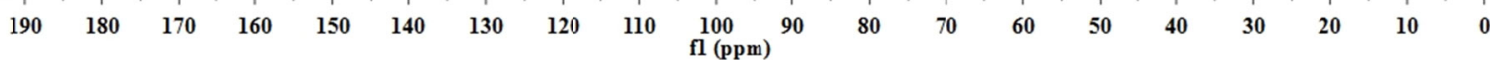




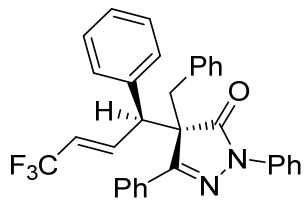

4a ${ }^{19} \mathrm{~F} \mathrm{NMR}\left(\mathrm{CDCl}_{3}, 377 \mathrm{MHz}\right)$
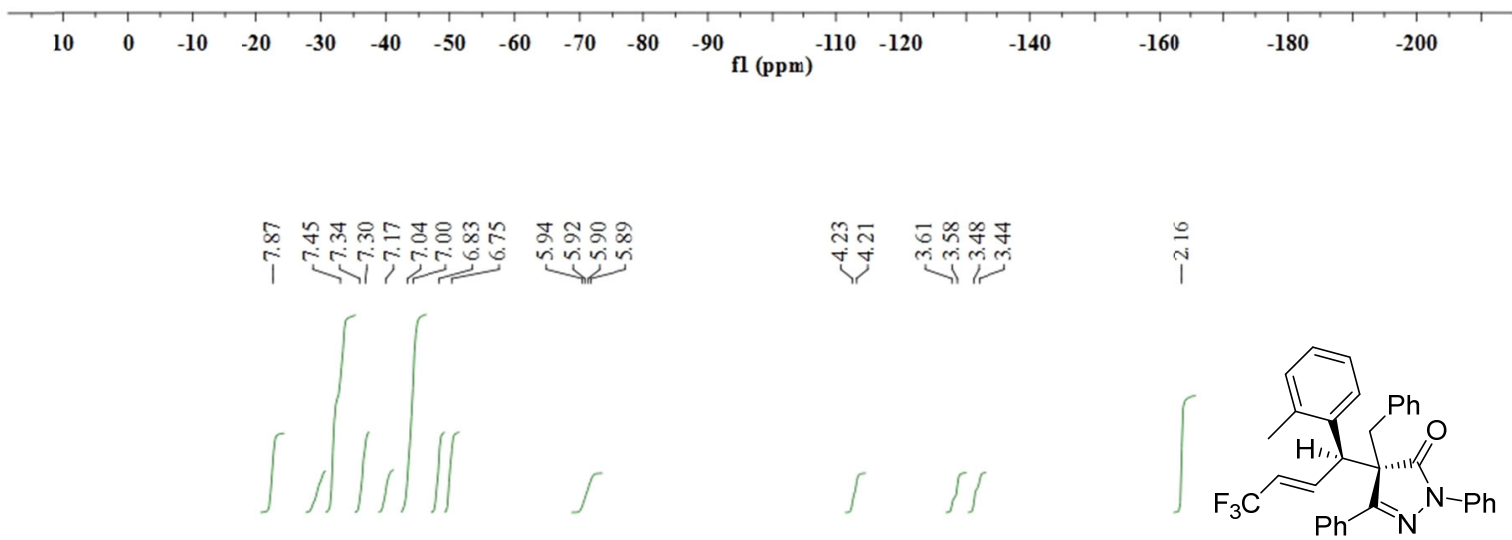

4b ${ }^{1} \mathrm{H} \mathrm{NMR}\left(\mathrm{CDCl}_{3}, 400 \mathrm{MHz}\right)$

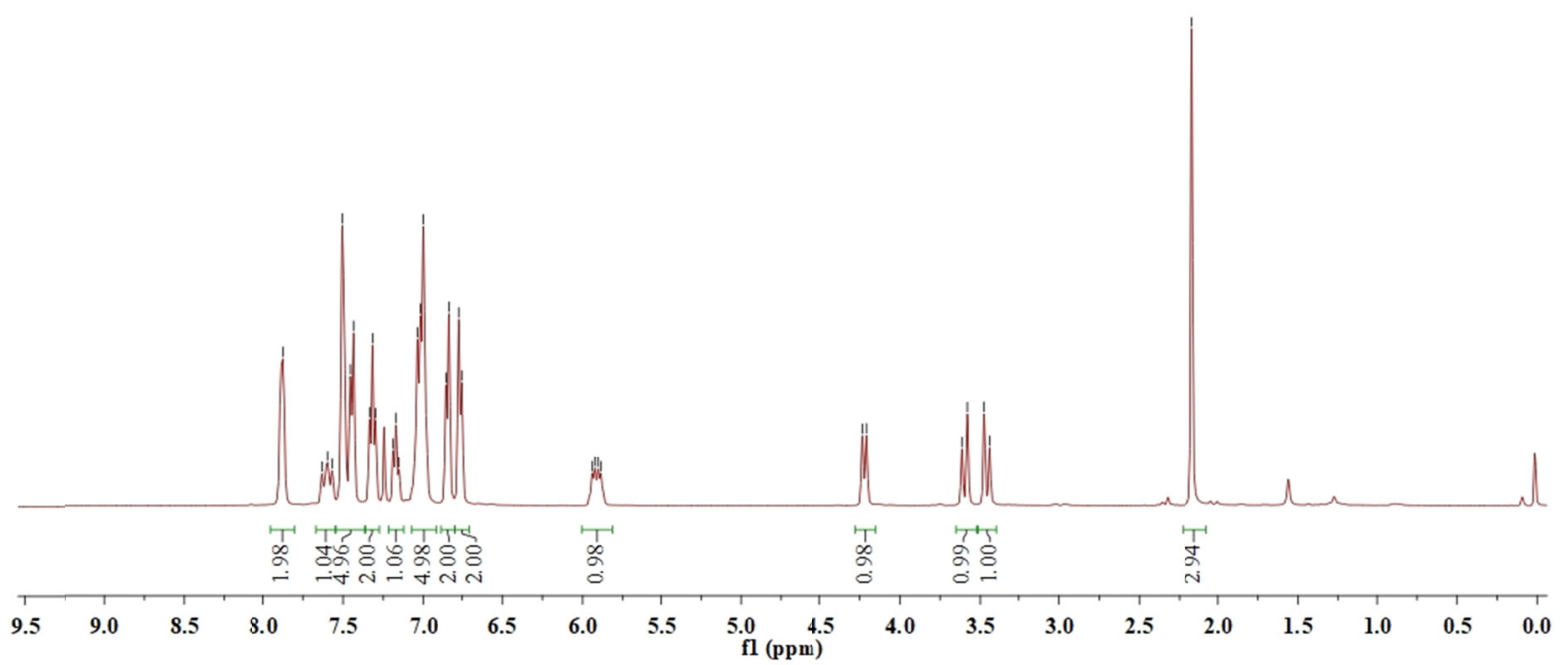



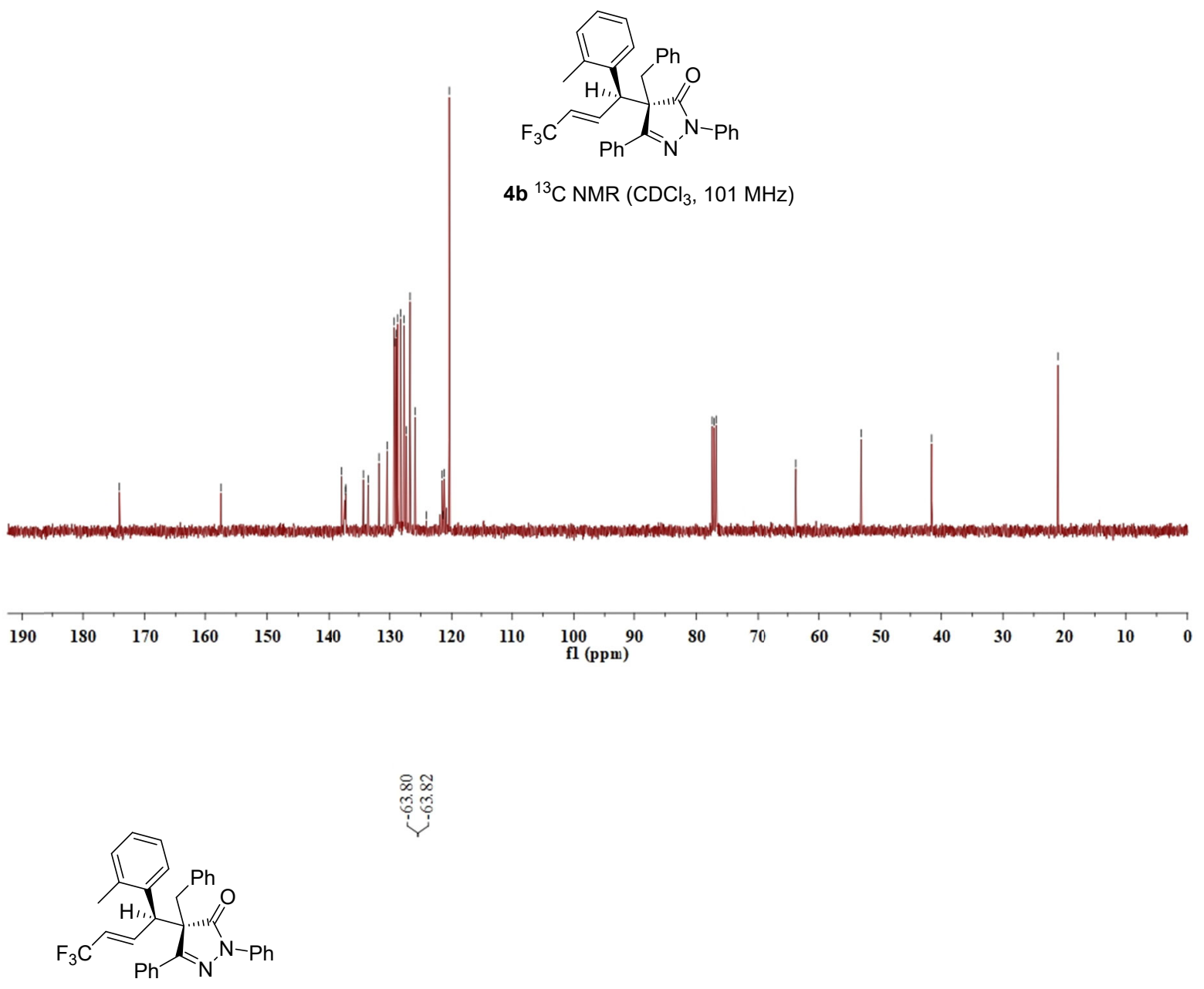

ن̃

4b ${ }^{19} \mathrm{~F} \mathrm{NMR}\left(\mathrm{CDCl}_{3}, 377 \mathrm{MHz}\right)$

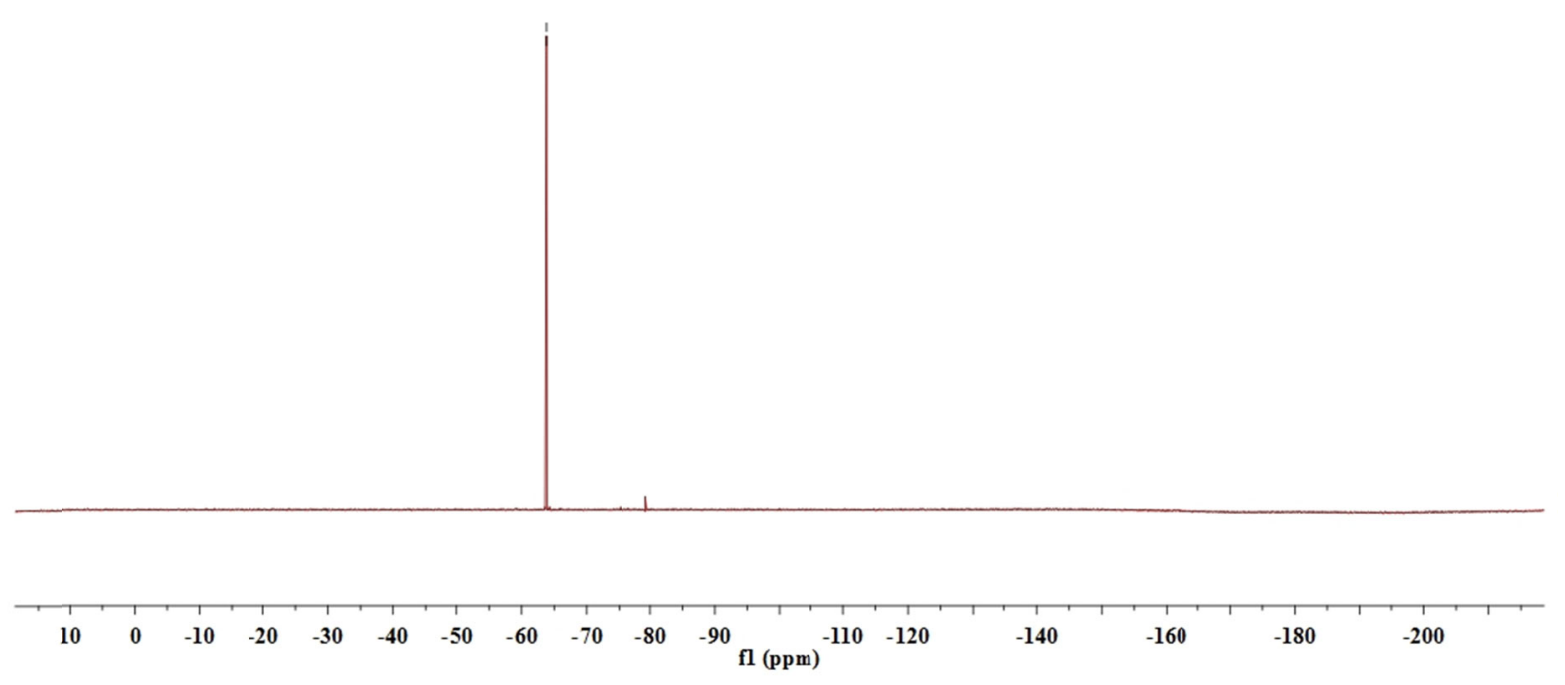




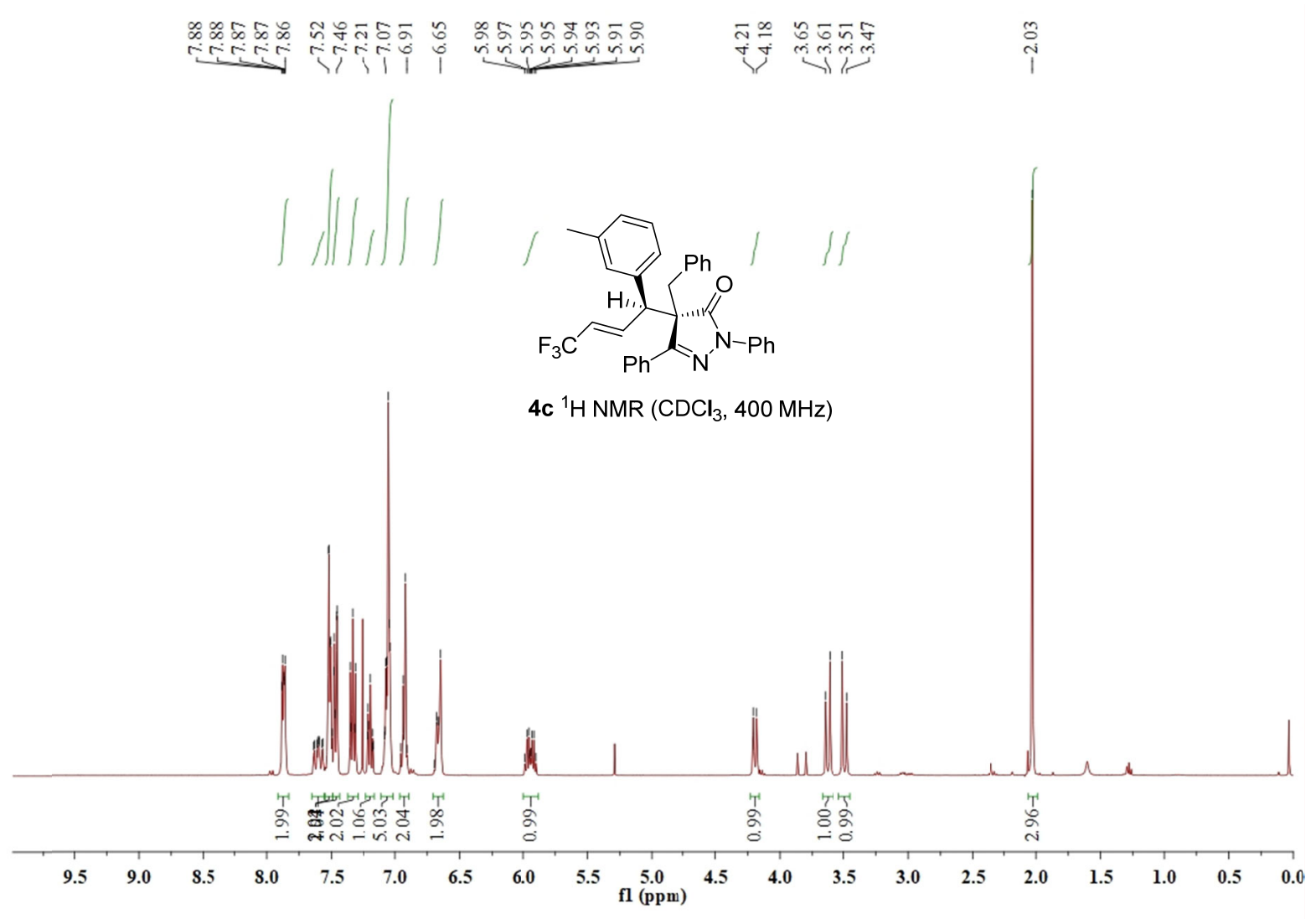

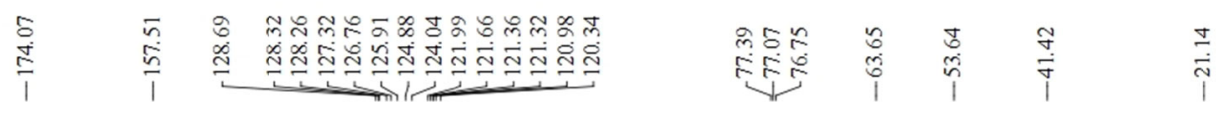

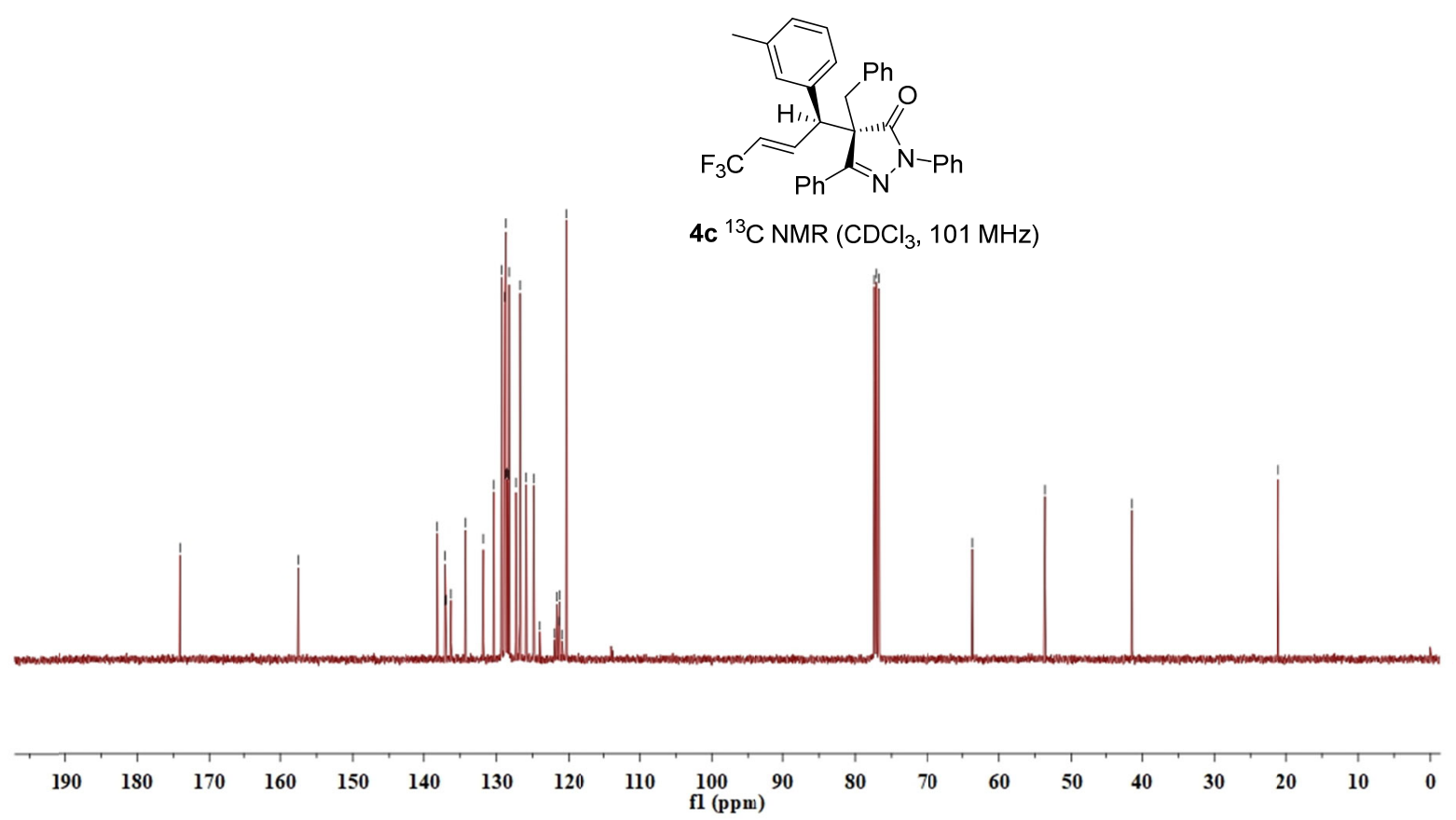




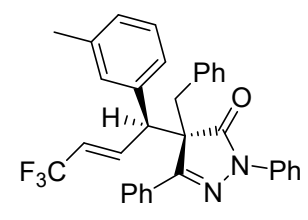

4c ${ }^{19} \mathrm{~F} \mathrm{NMR}\left(\mathrm{CDCl}_{3}, 377 \mathrm{MHz}\right)$
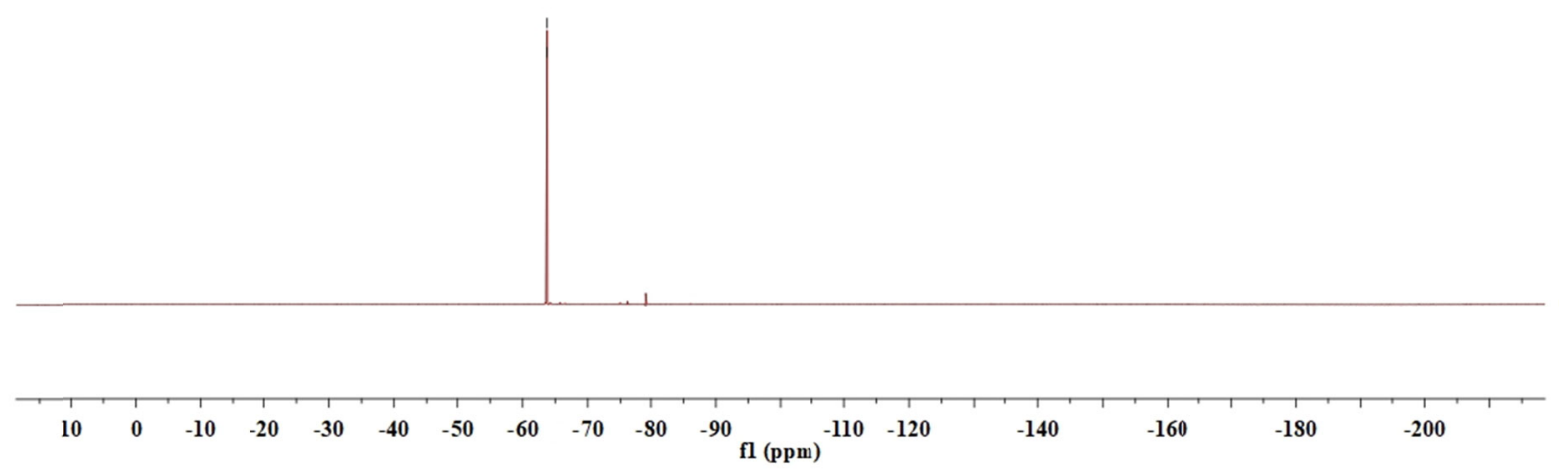

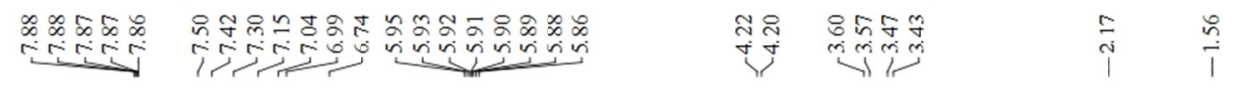

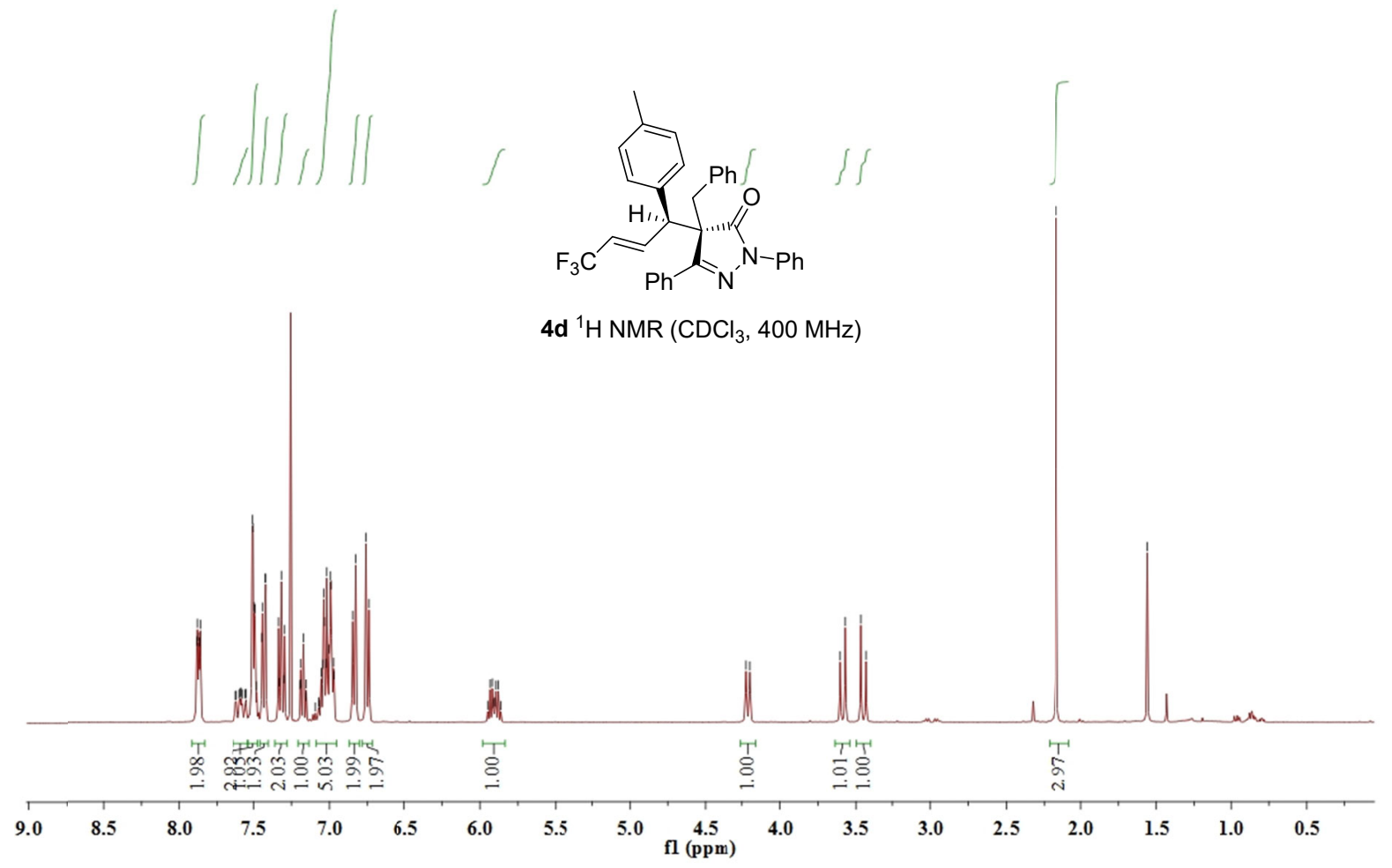




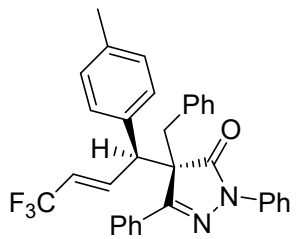

4d ${ }^{13} \mathrm{C} \mathrm{NMR}\left(\mathrm{CDCl}_{3}, 101 \mathrm{MHz}\right)$

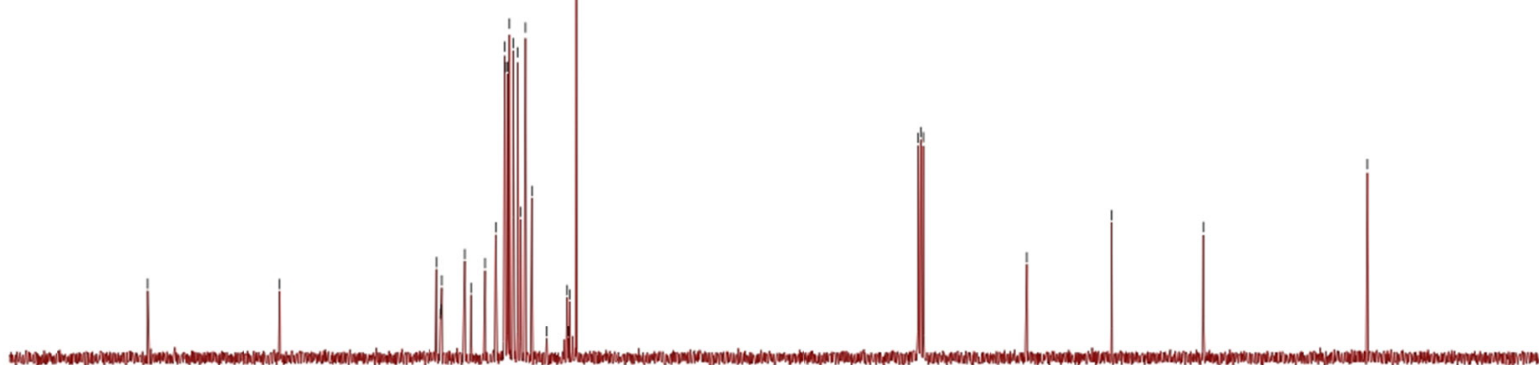

$\begin{array}{llllllllllllllllllll}190 & 180 & 170 & 160 & 150 & 140 & 130 & 120 & 110 & 100 & 90 & 80 & 70 & 60 & 50 & 40 & 30 & 20 & 10 & 0\end{array}$

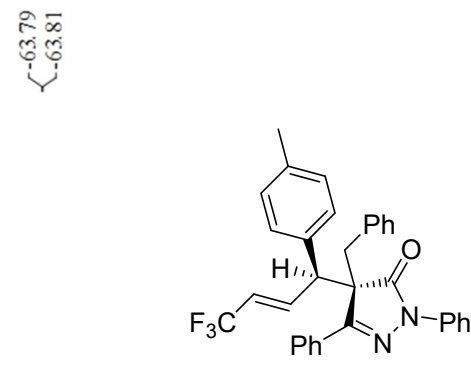

4d ${ }^{19} \mathrm{~F} \mathrm{NMR}\left(\mathrm{CDCl}_{3}, 377 \mathrm{MHz}\right)$

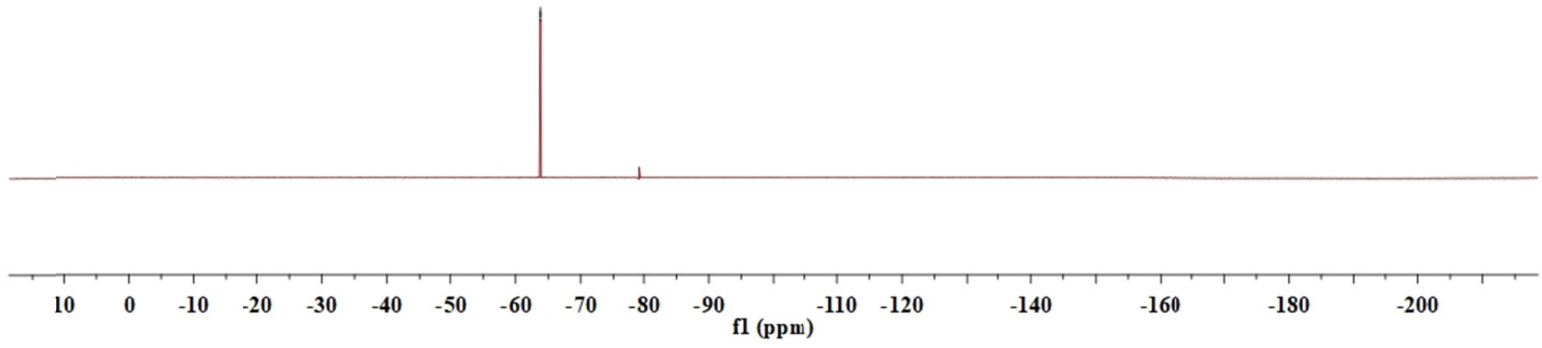




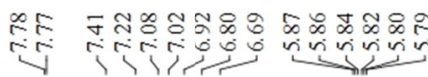

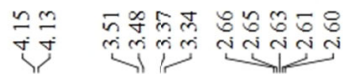

$\underset{-}{-8}$
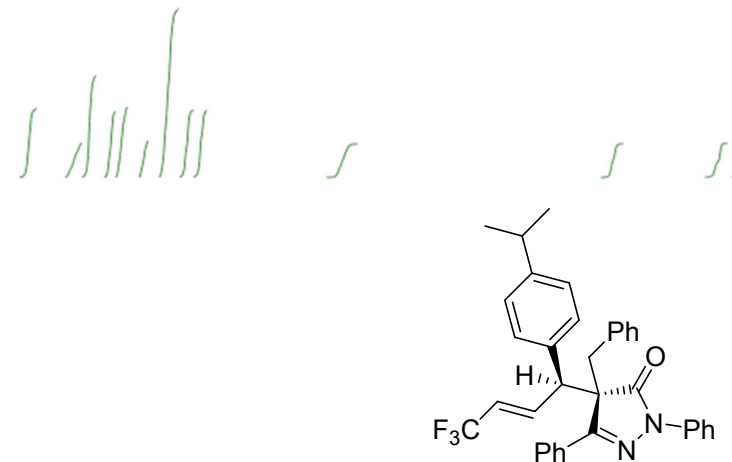

4e ${ }^{1} \mathrm{H} \mathrm{NMR}\left(\mathrm{CDCl}_{3}, 400 \mathrm{MHz}\right)$

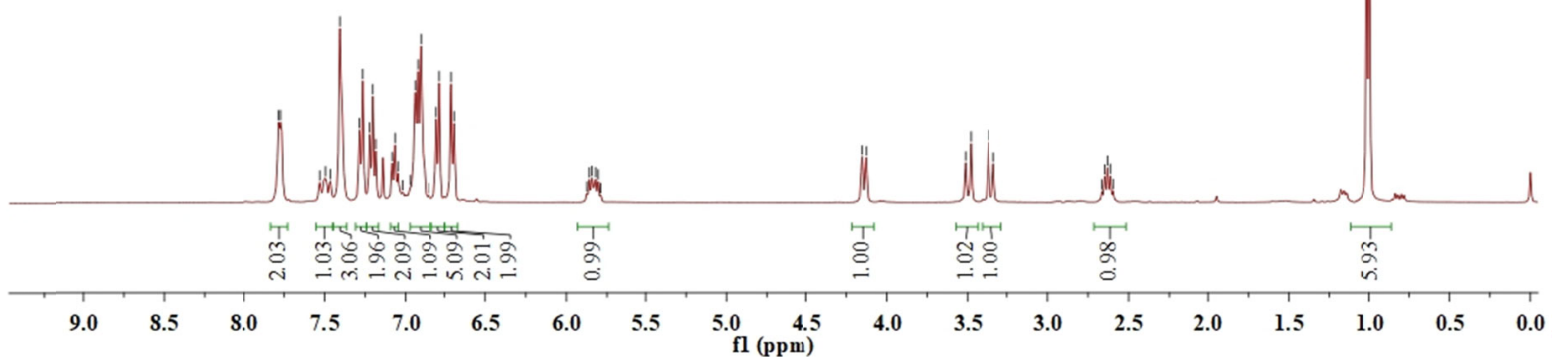

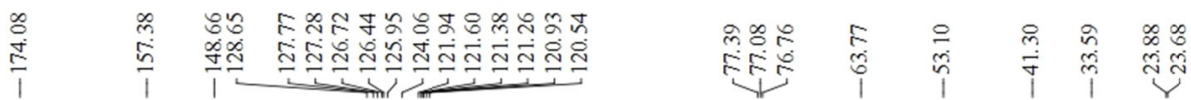

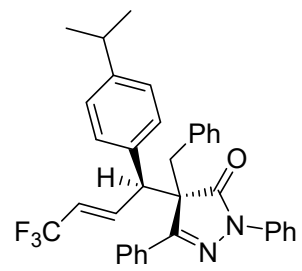

4e ${ }^{13} \mathrm{C}$ NMR $\left(\mathrm{CDCl}_{3}, 101 \mathrm{MHz}\right)$

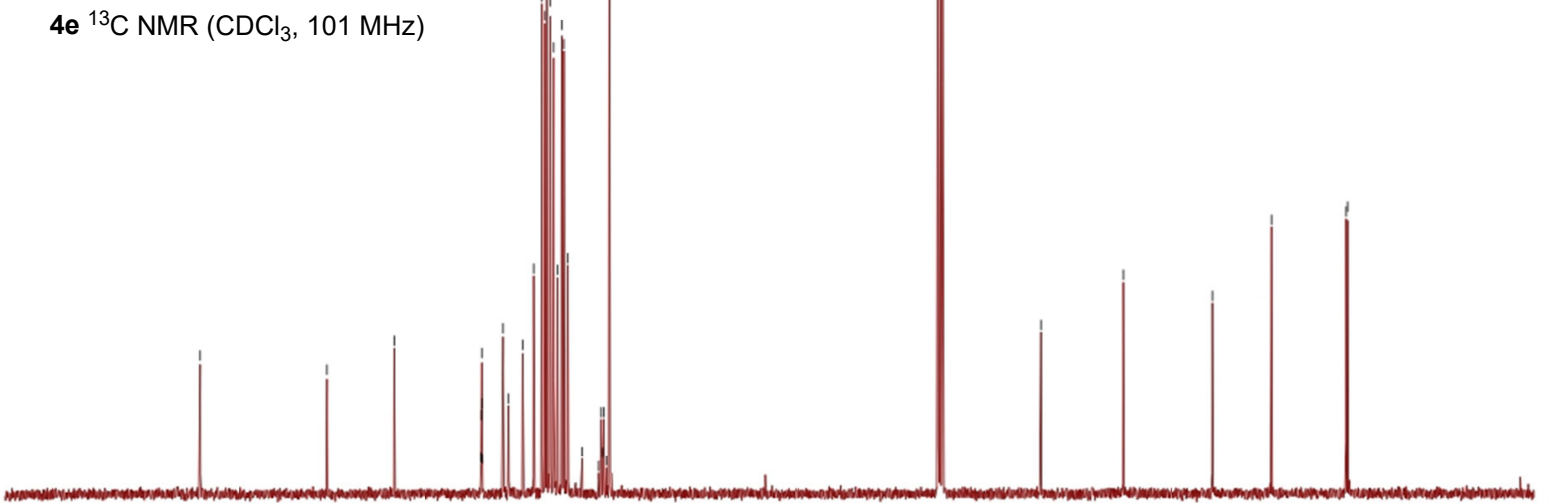

$\begin{array}{lllllllllllllllllllll}190 & 180 & 170 & 160 & 150 & 140 & 130 & 120 & 110 & \begin{array}{c}100 \\ \mathrm{fl}(\mathrm{ppm})\end{array} & 90 & \mathbf{8 0} & 70 & 60 & 50 & 40 & 30 & 20 & 10 & 0\end{array}$ 


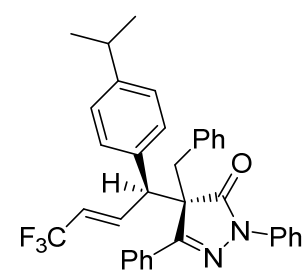

4e ${ }^{19} \mathrm{~F} \mathrm{NMR}\left(\mathrm{CDCl}_{3}, 377 \mathrm{MHz}\right)$

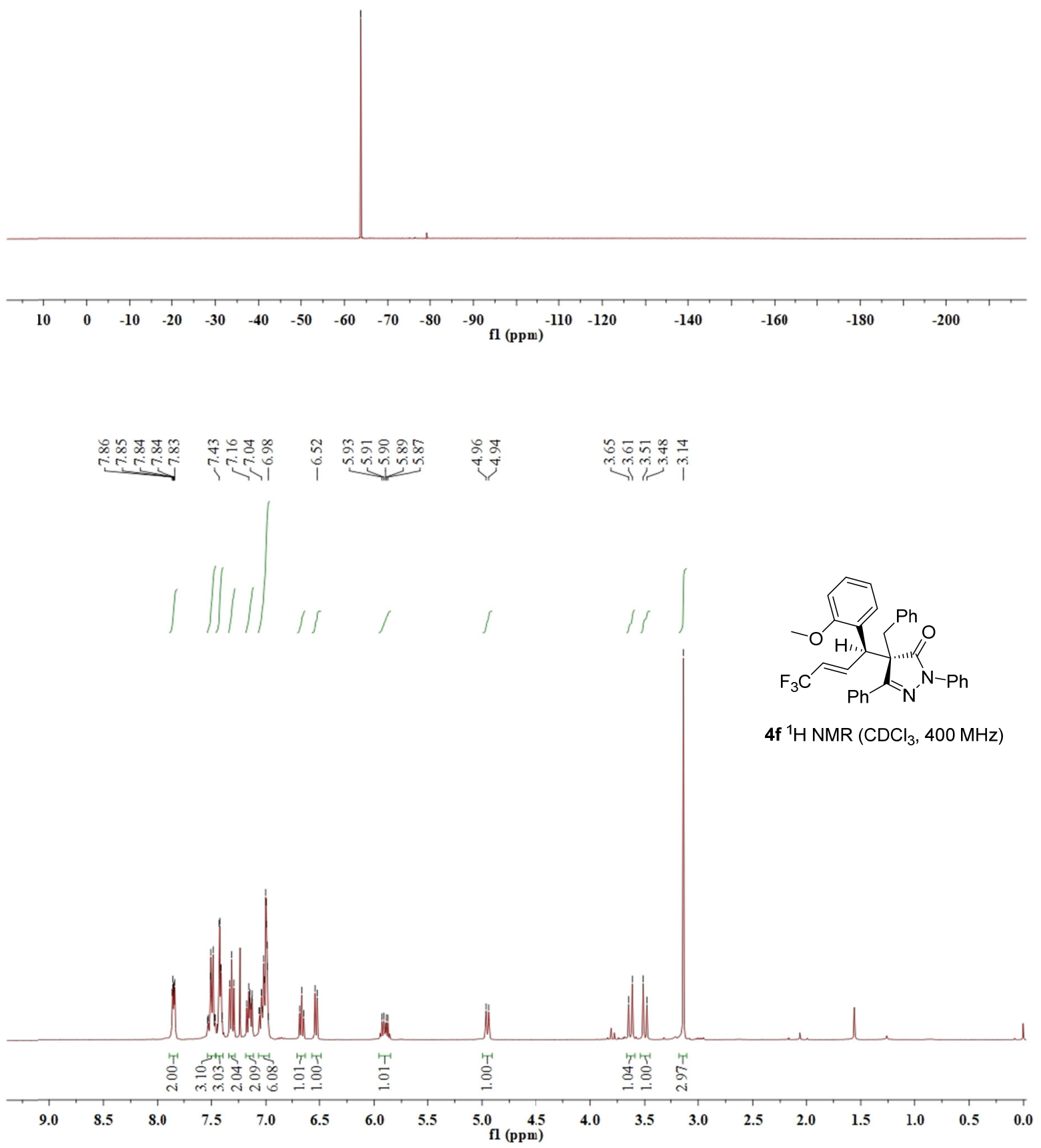




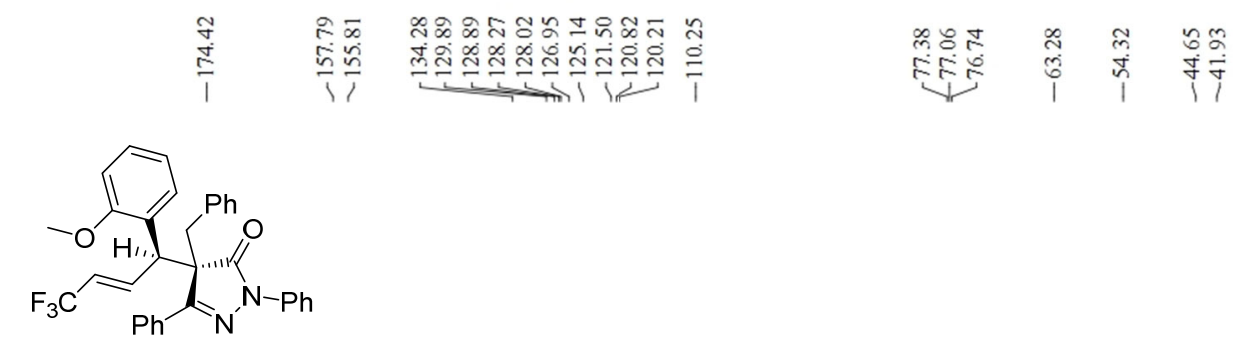

4f ${ }^{13} \mathrm{C} \mathrm{NMR}\left(\mathrm{CDCl}_{3}, 101 \mathrm{MHz}\right)$
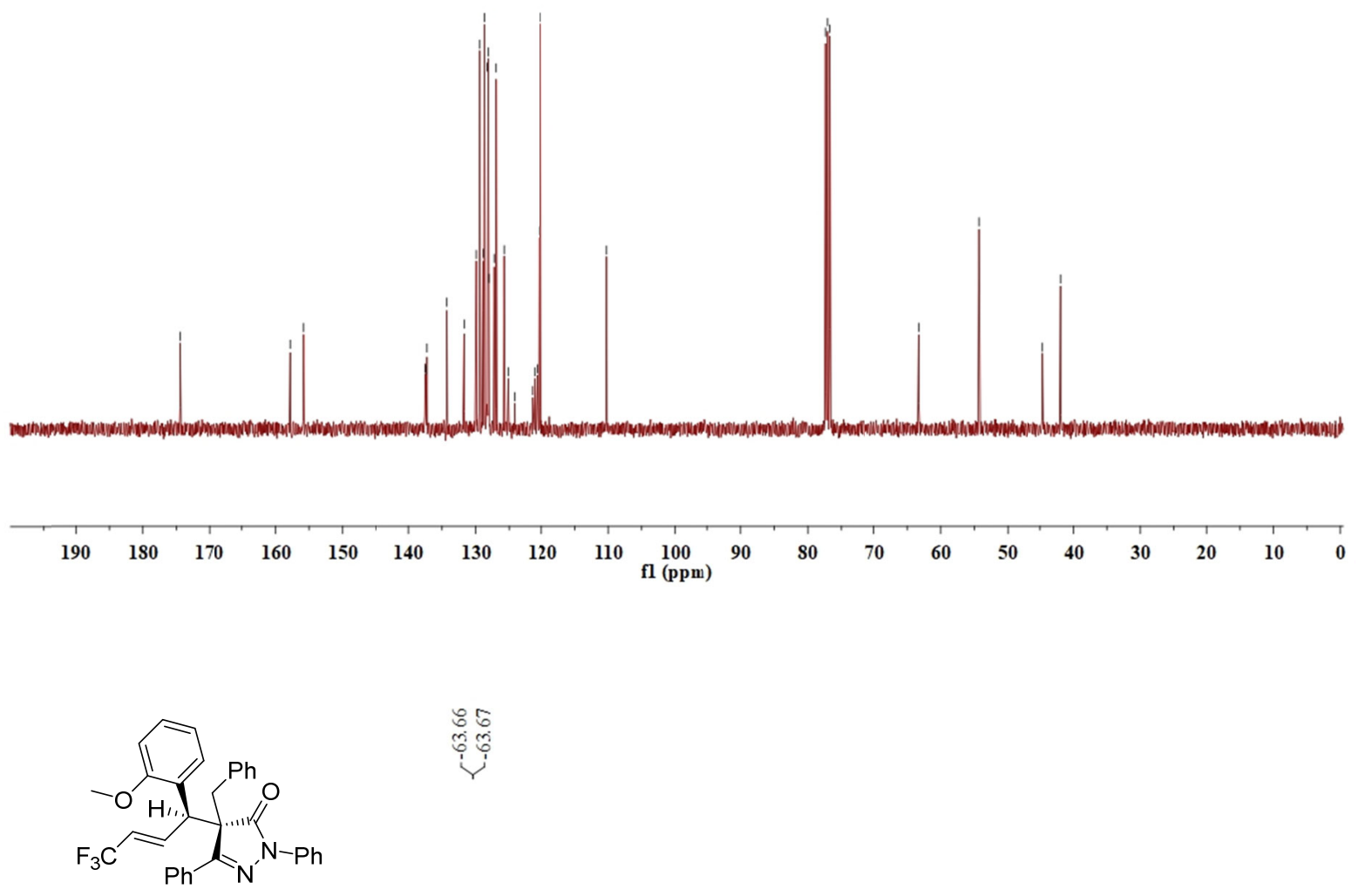

ن

4f ${ }^{19} \mathrm{~F} \mathrm{NMR}\left(\mathrm{CDCl}_{3}, 377 \mathrm{MHz}\right)$

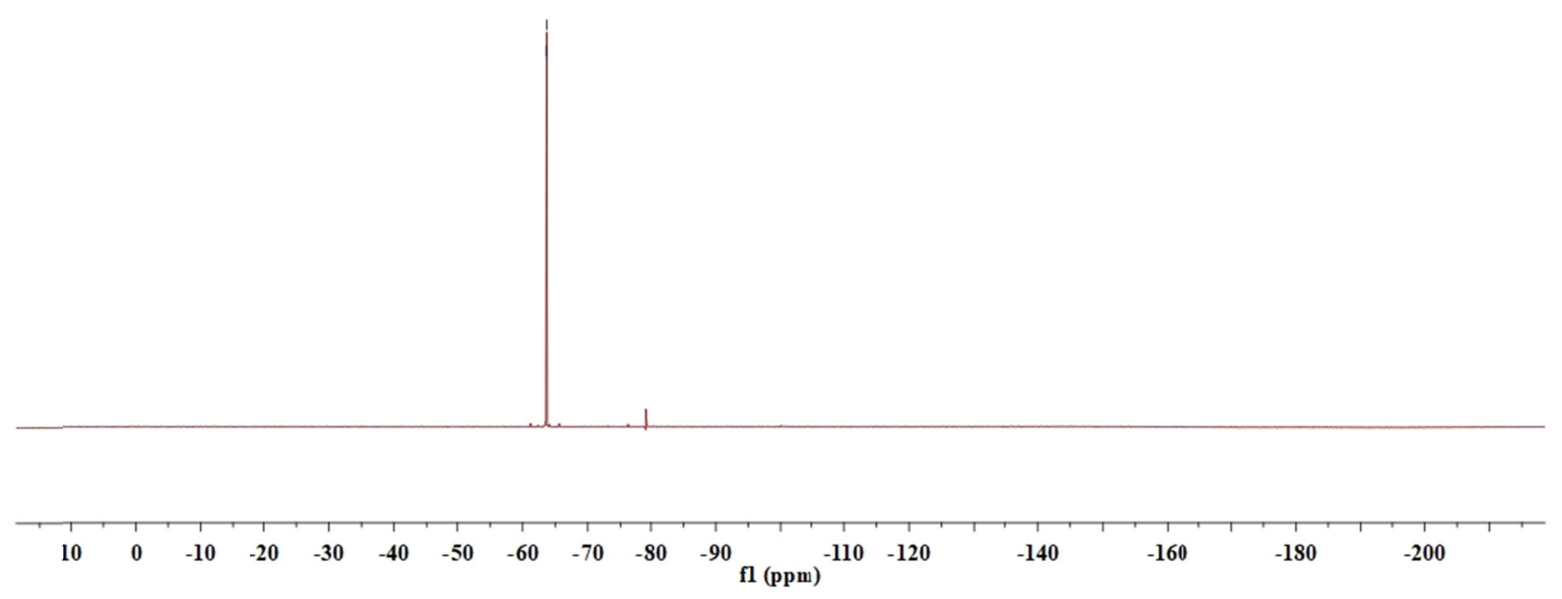




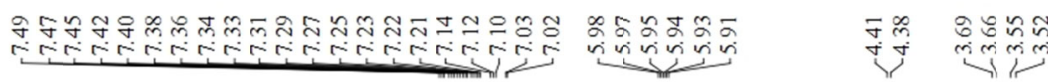

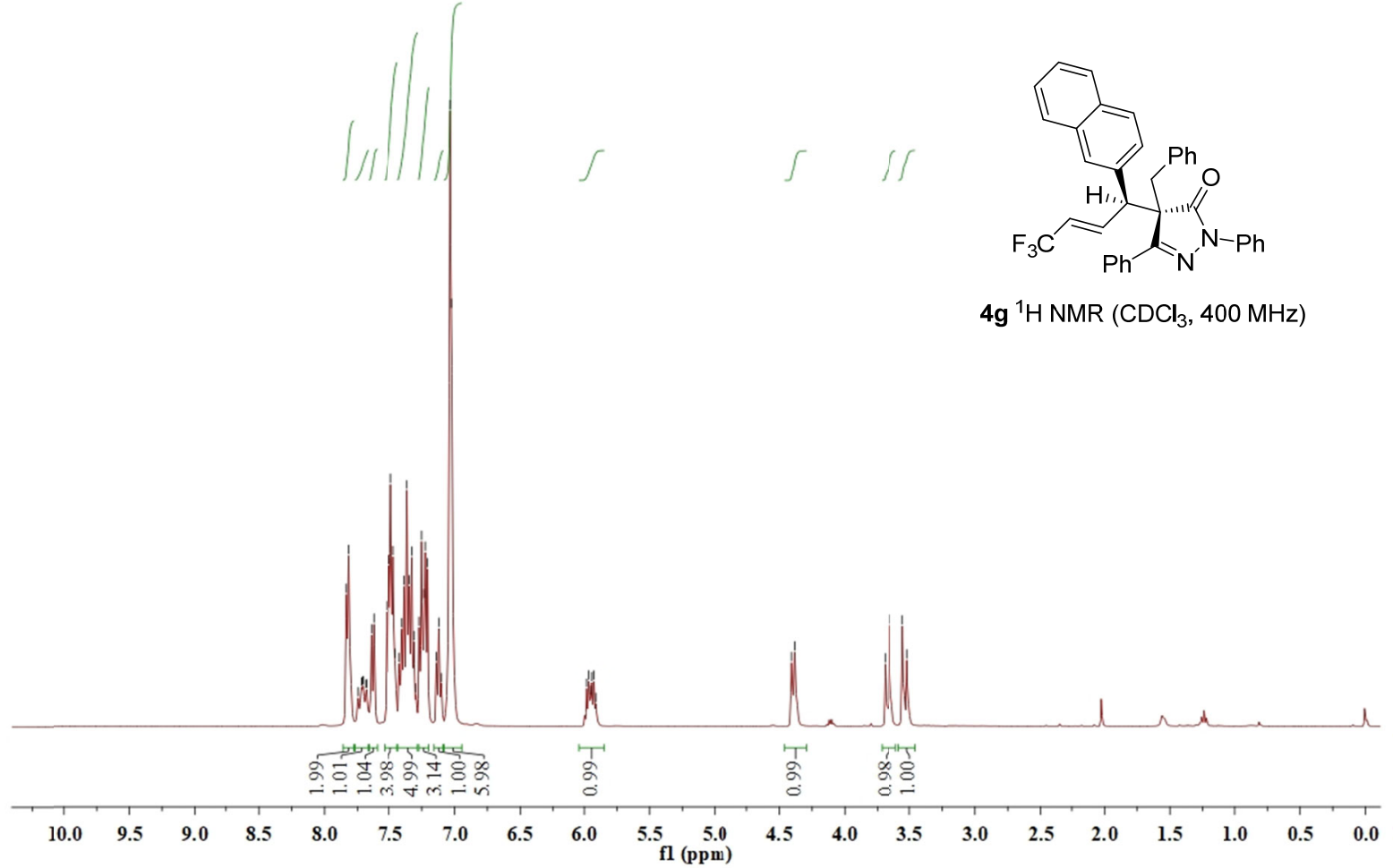

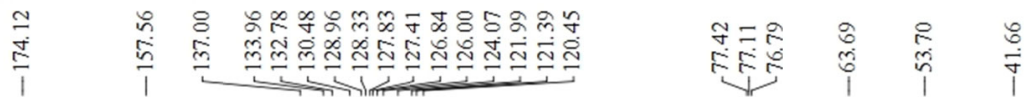

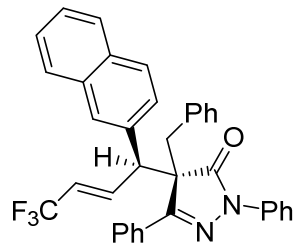

$\mathbf{4 g}{ }^{13} \mathrm{C} \mathrm{NMR}\left(\mathrm{CDCl}_{3}, 101 \mathrm{MHz}\right)$

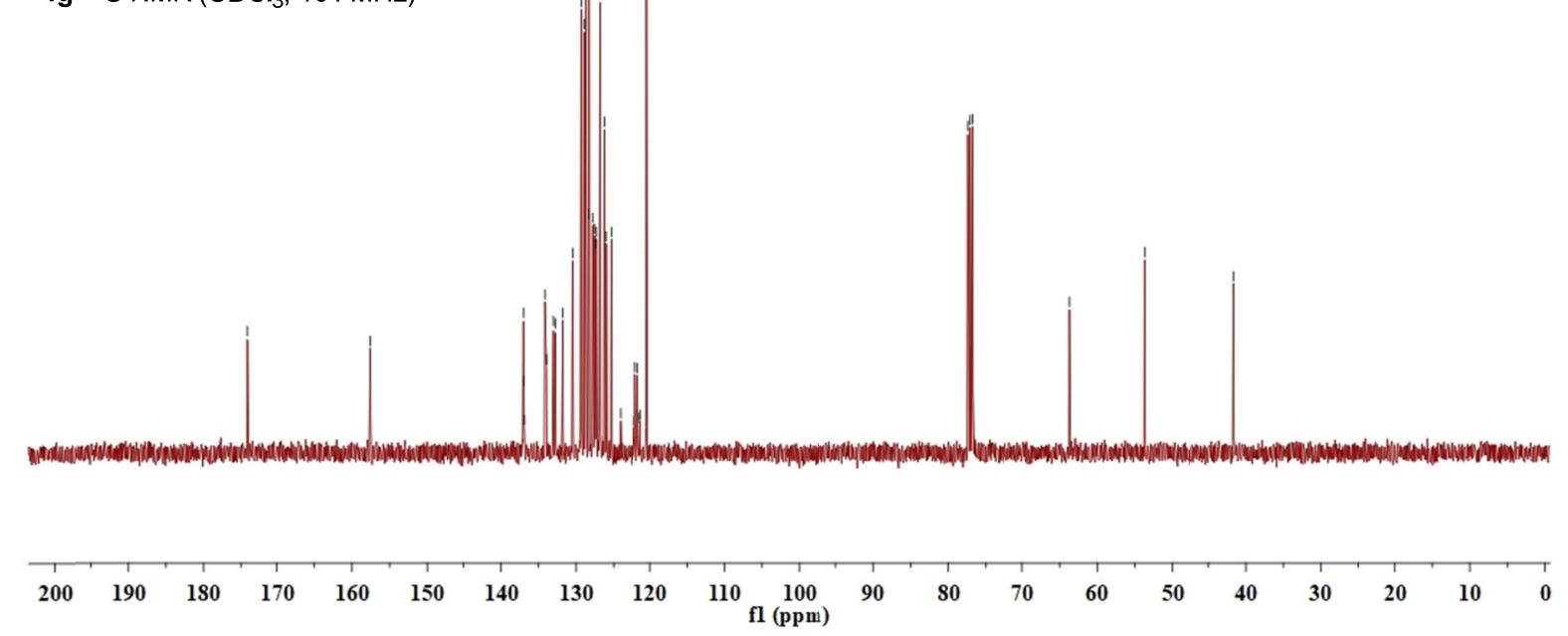




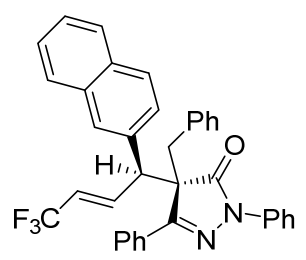

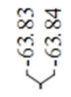

4g ${ }^{19} \mathrm{~F}$ NMR $\left(\mathrm{CDCl}_{3}, 377 \mathrm{MHz}\right)$

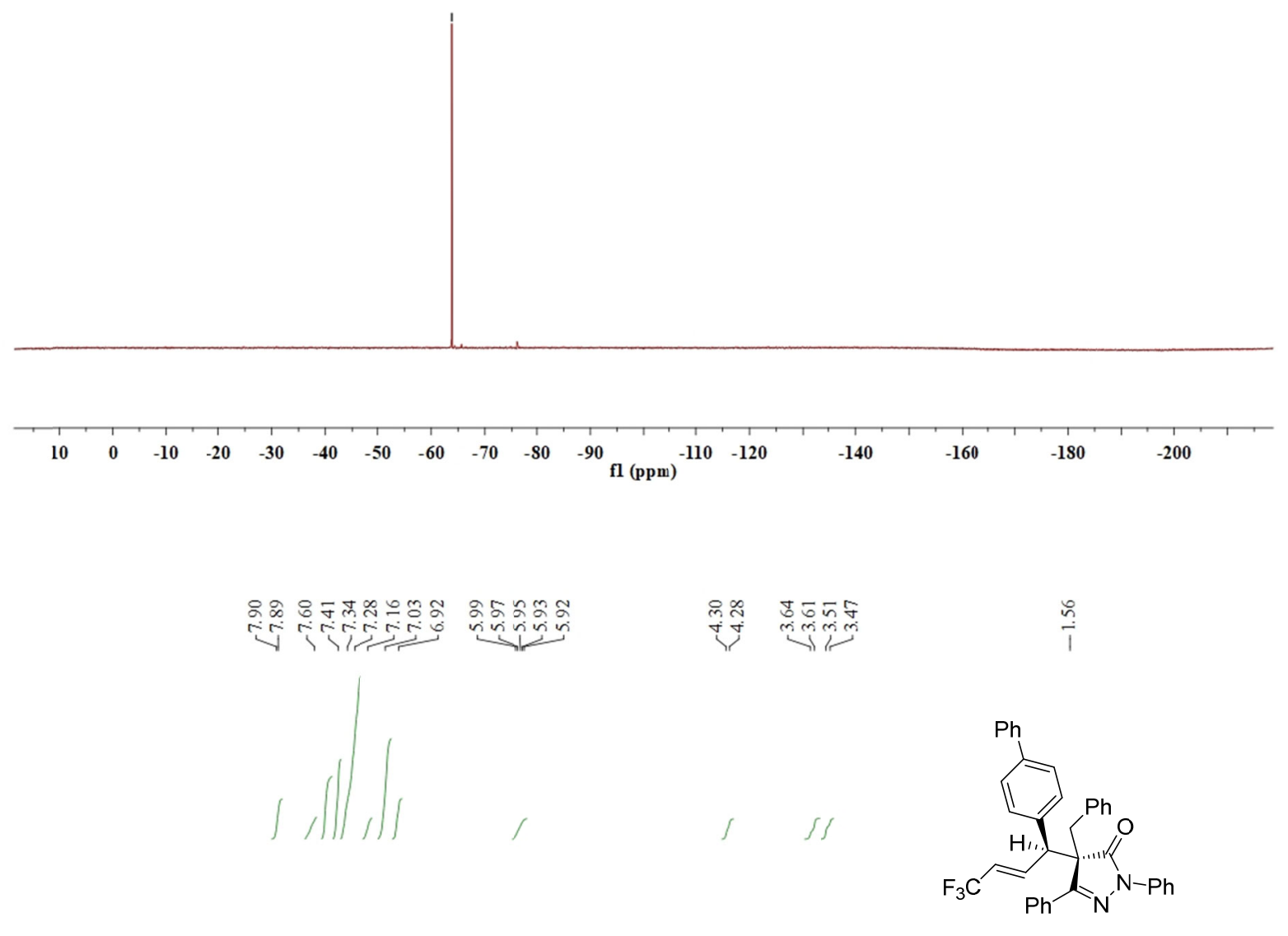

4h ${ }^{1} \mathrm{H} \mathrm{NMR}\left(\mathrm{CDCl}_{3}, 400 \mathrm{MHz}\right)$

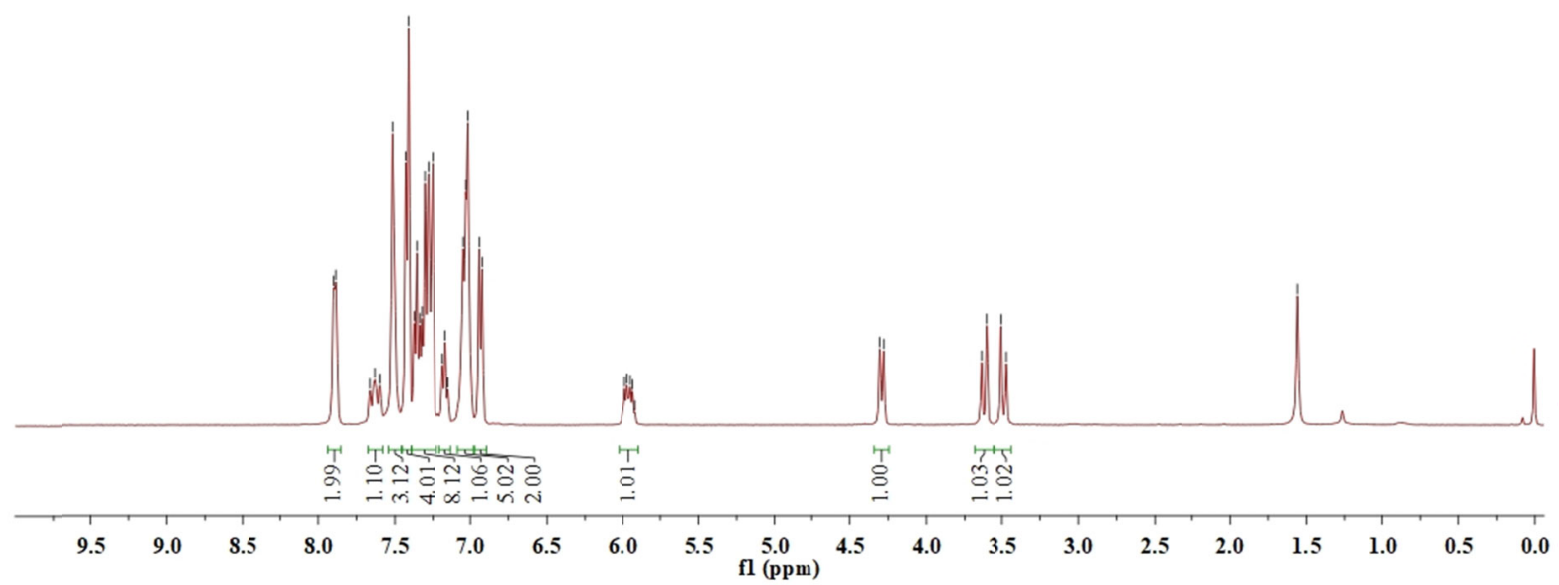




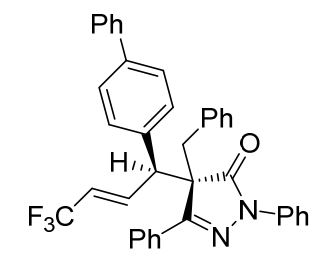

4h ${ }^{13} \mathrm{C} \mathrm{NMR}\left(\mathrm{CDCl}_{3}, 101 \mathrm{MHz}\right)$
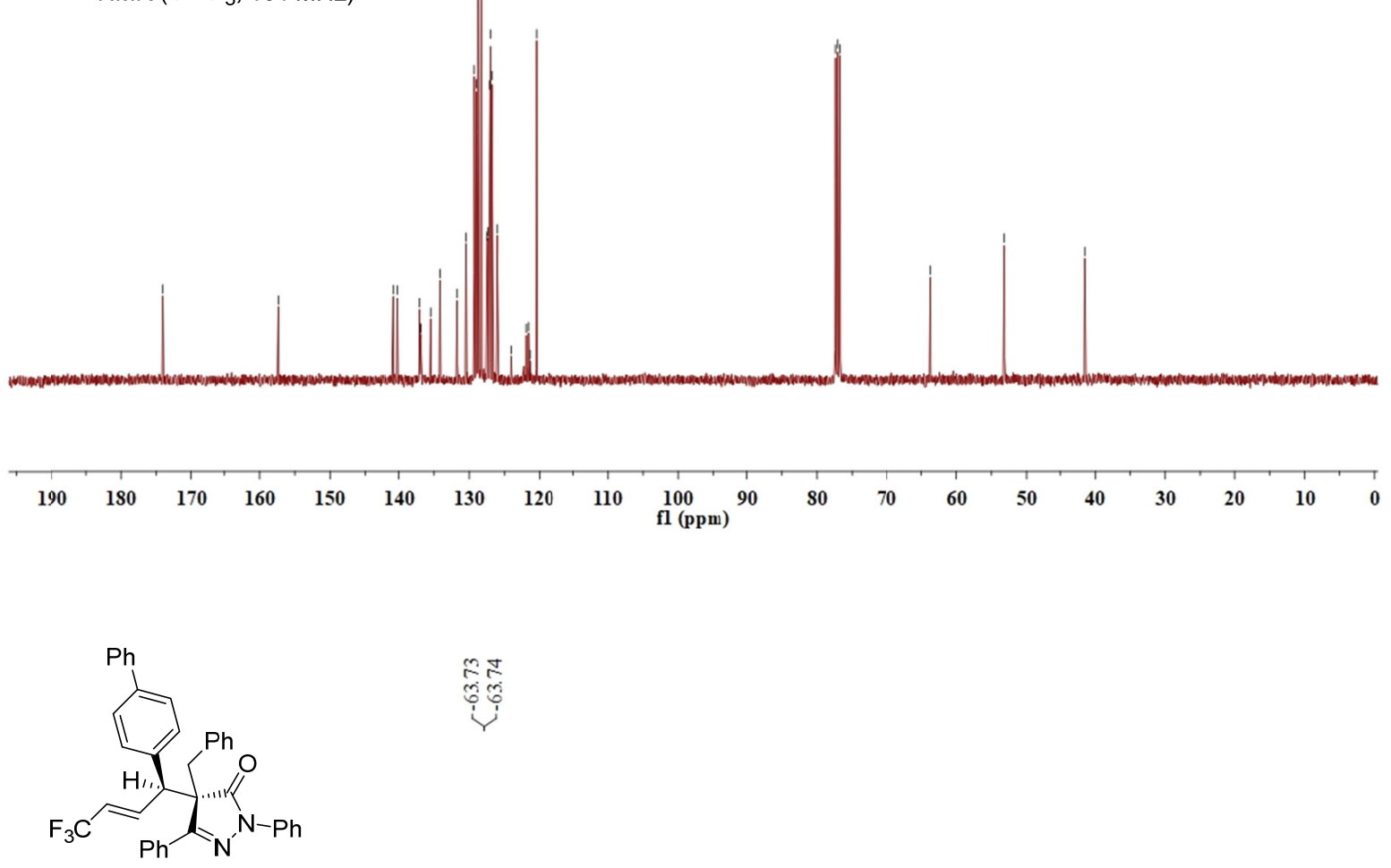

în

4h ${ }^{19} \mathrm{~F} \mathrm{NMR}\left(\mathrm{CDCl}_{3}, 377 \mathrm{MHz}\right)$

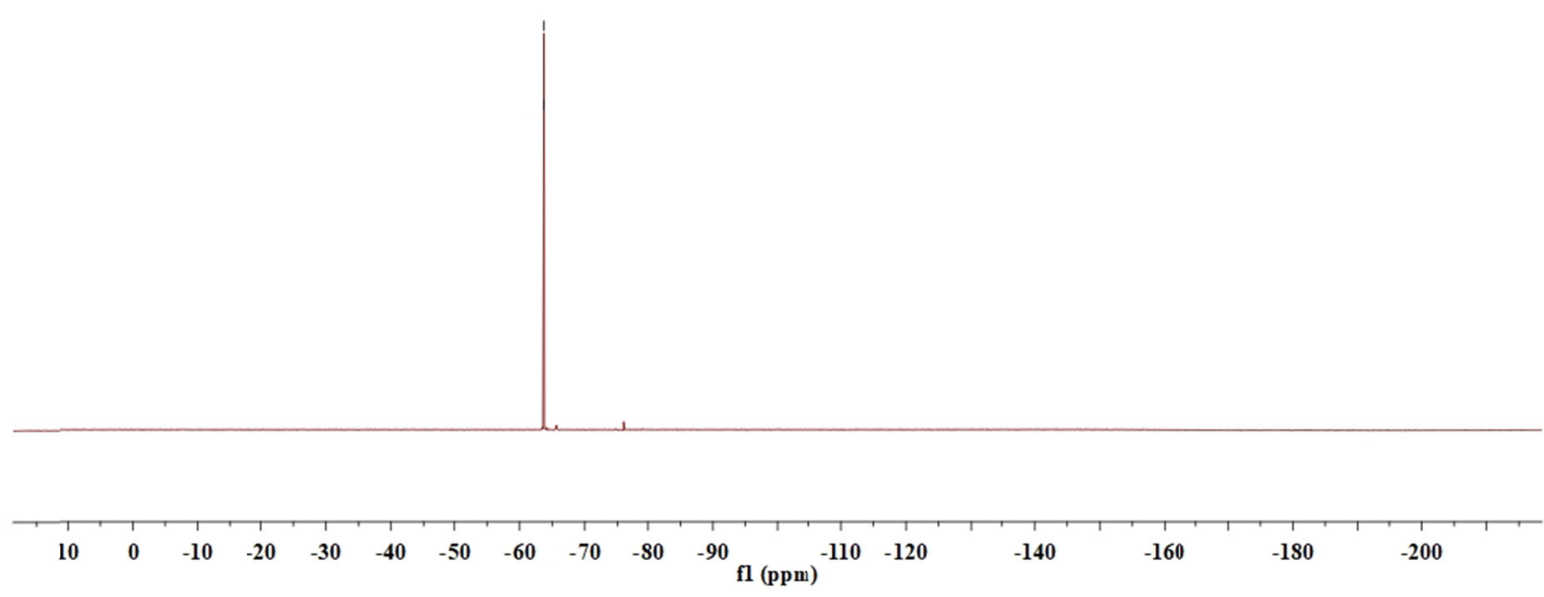


$\underbrace{\infty}$

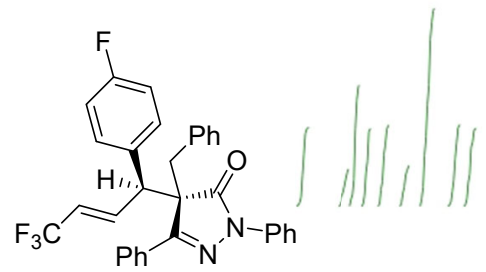

4i ${ }^{1} \mathrm{H}$ NMR $\left(\mathrm{CDCl}_{3}, 400 \mathrm{MHz}\right)$

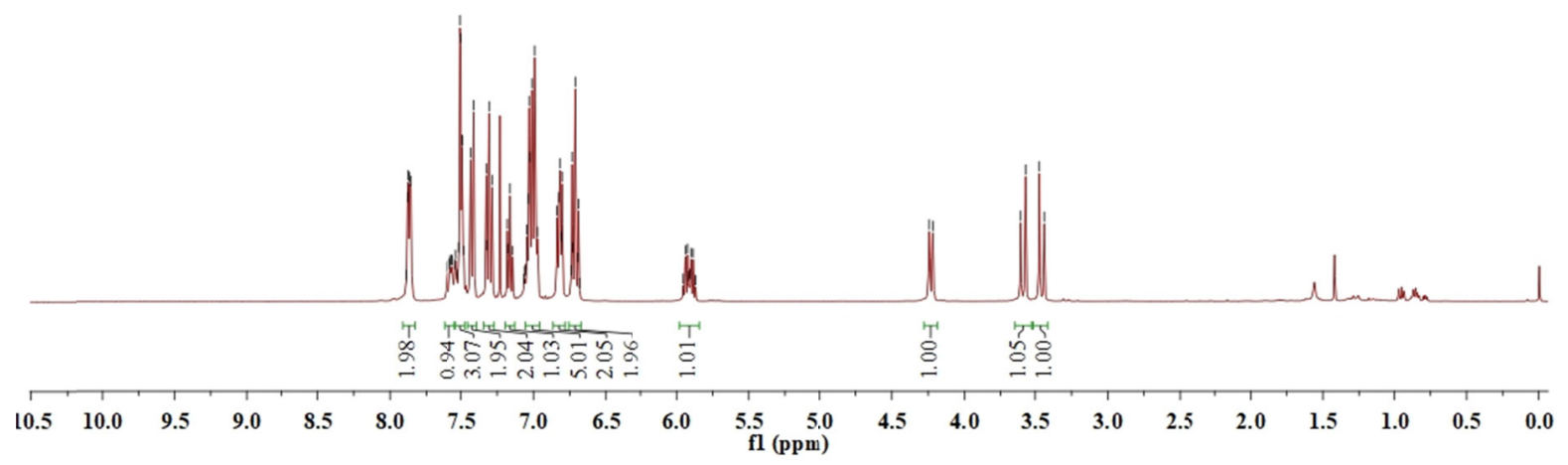

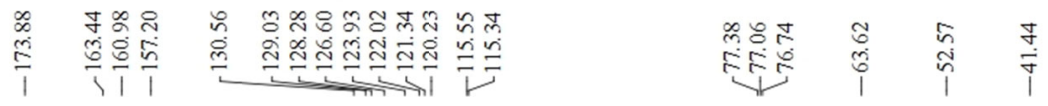

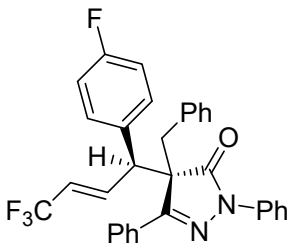

$4 \mathbf{i}^{13} \mathrm{C}$ NMR $\left(\mathrm{CDCl}_{3}, 101 \mathrm{MHz}\right)$

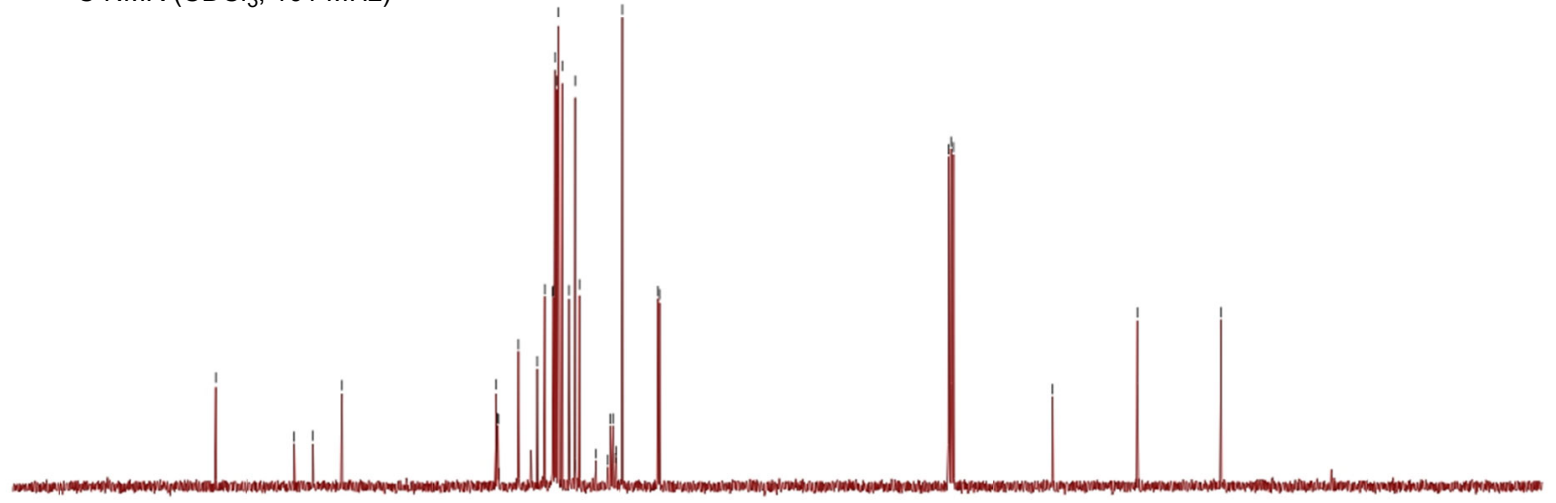

200

$190 \quad 180$

$170 \quad 160 \quad 150$

$\begin{array}{llll}50 & 140 & 130 & 120\end{array}$

$110 \underset{\mathrm{fl}(\mathrm{ppm})}{100} 9$

80

6050

$\begin{array}{lllll}40 & 30 & 20 & 10 & 0\end{array}$ 


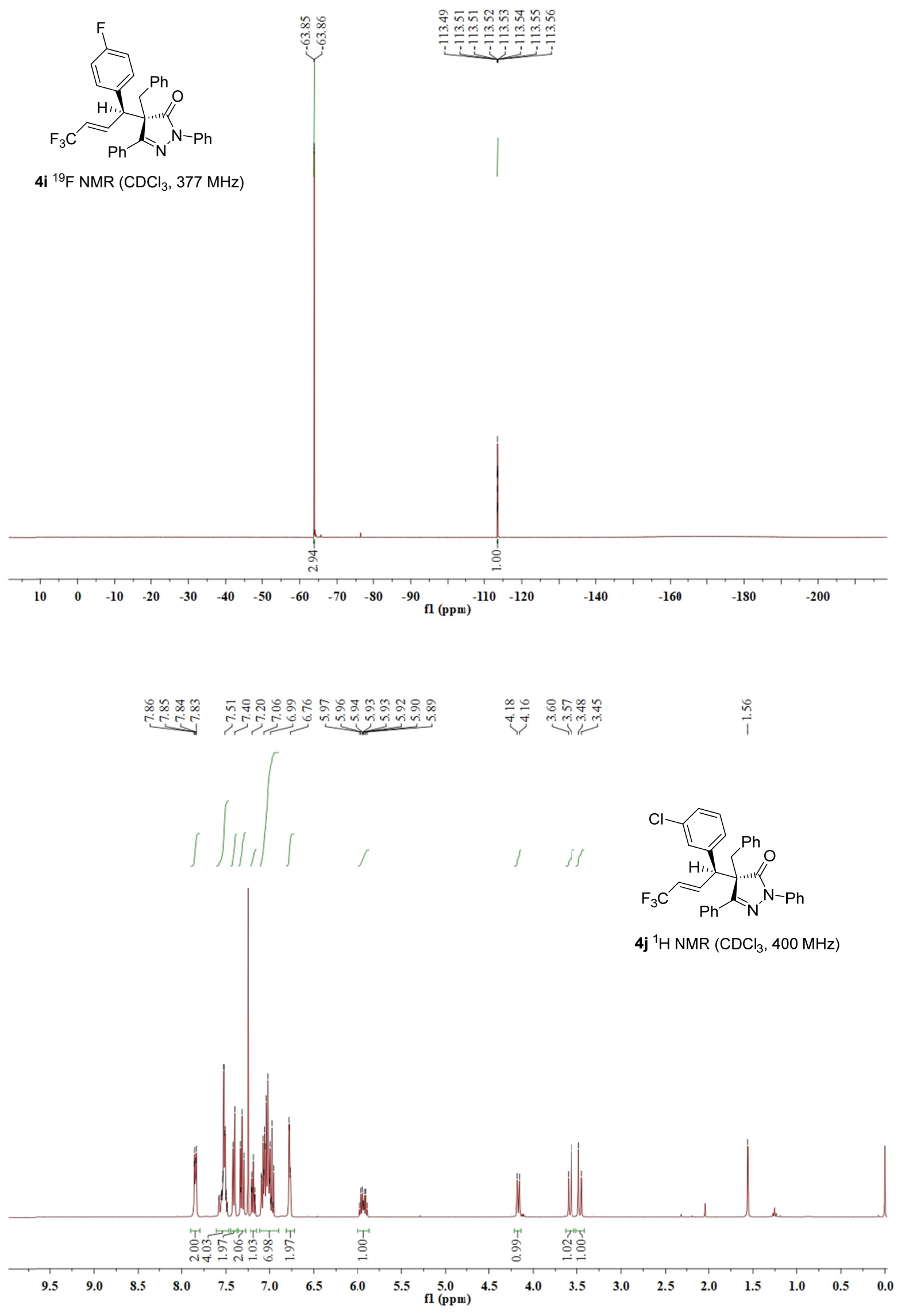




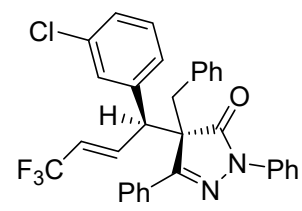

$4 \mathbf{j}^{13} \mathrm{C} \mathrm{NMR}\left(\mathrm{CDCl}_{3}, 101 \mathrm{MHz}\right)$
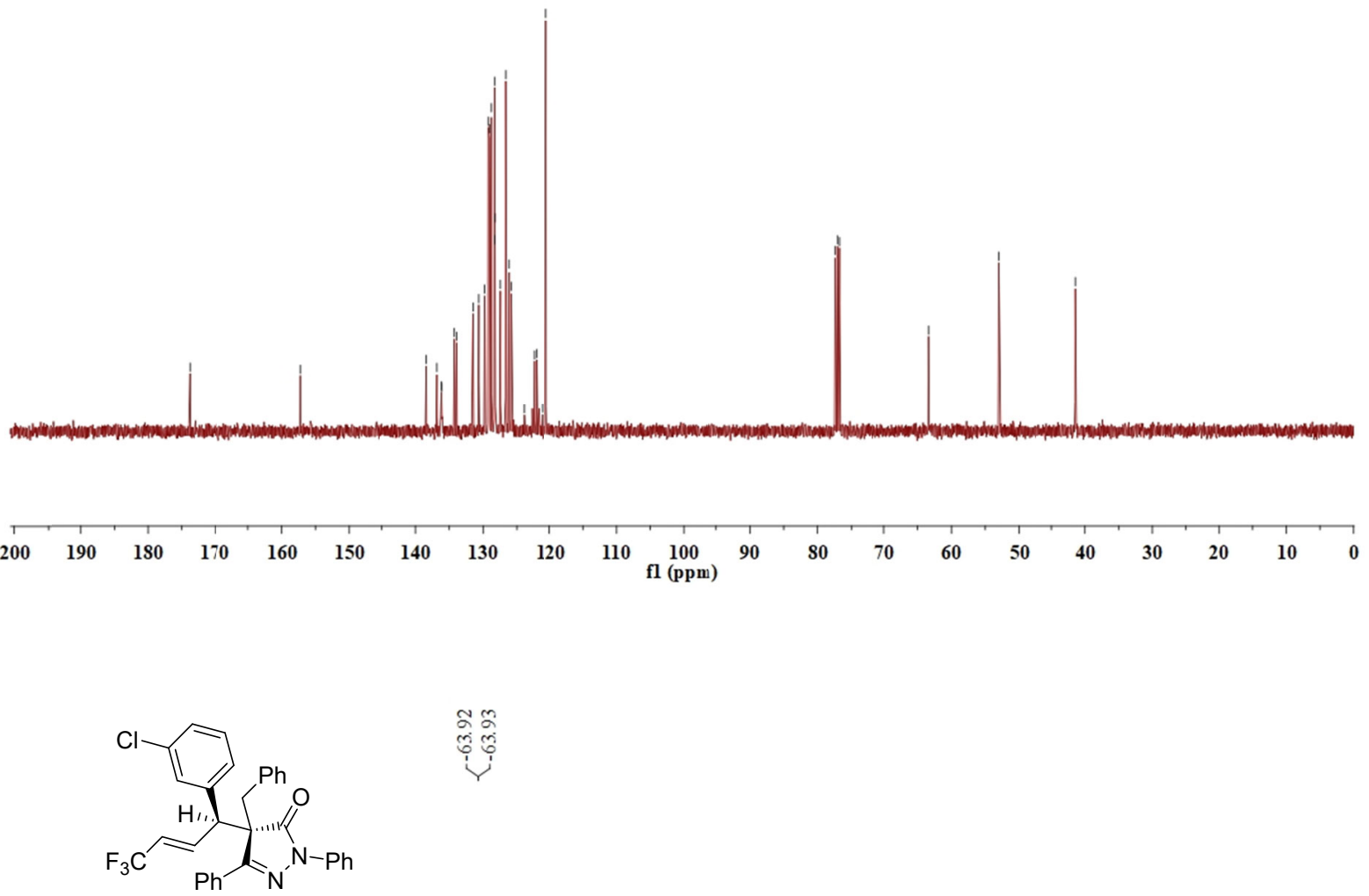

సूे

4j ${ }^{19} \mathrm{~F} \mathrm{NMR}\left(\mathrm{CDCl}_{3}, 377 \mathrm{MHz}\right)$

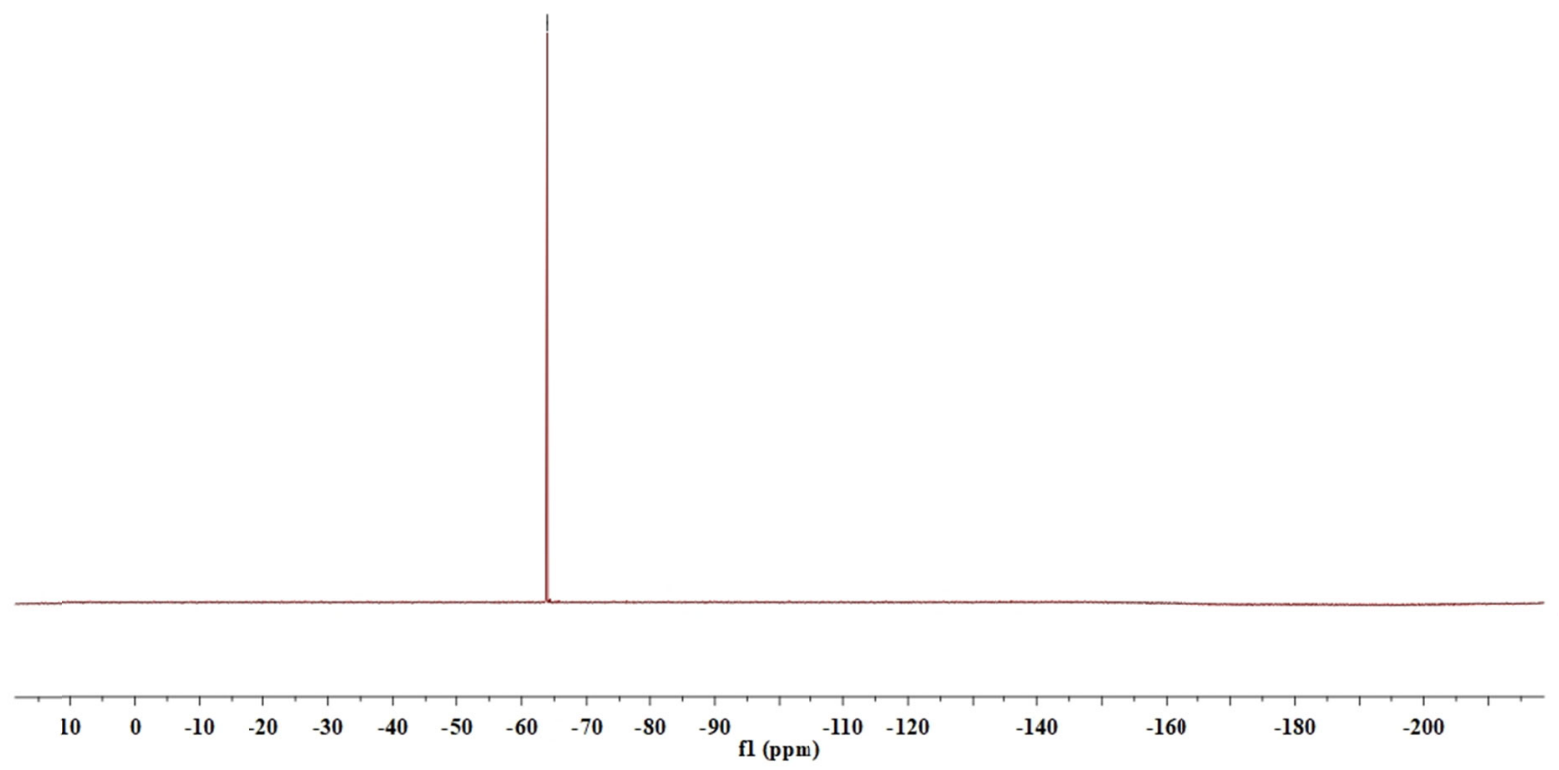




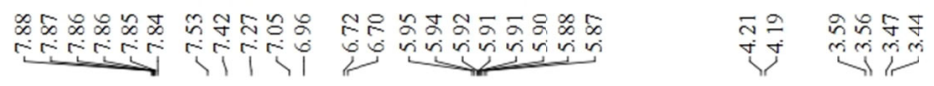
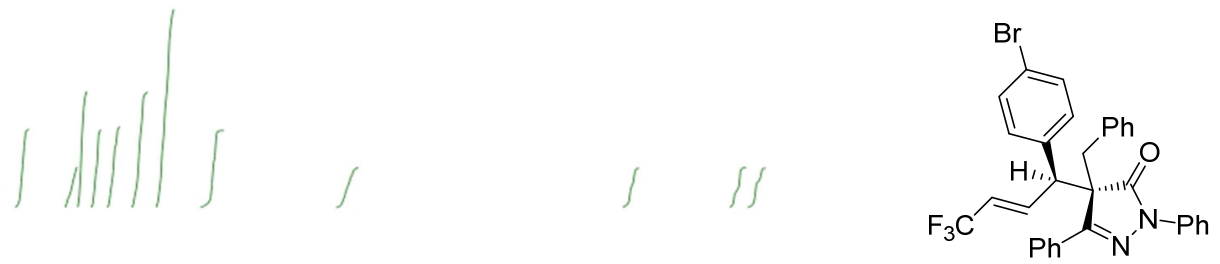

4k ${ }^{1} \mathrm{H}$ NMR $\left(\mathrm{CDCl}_{3}, 400 \mathrm{MHz}\right)$

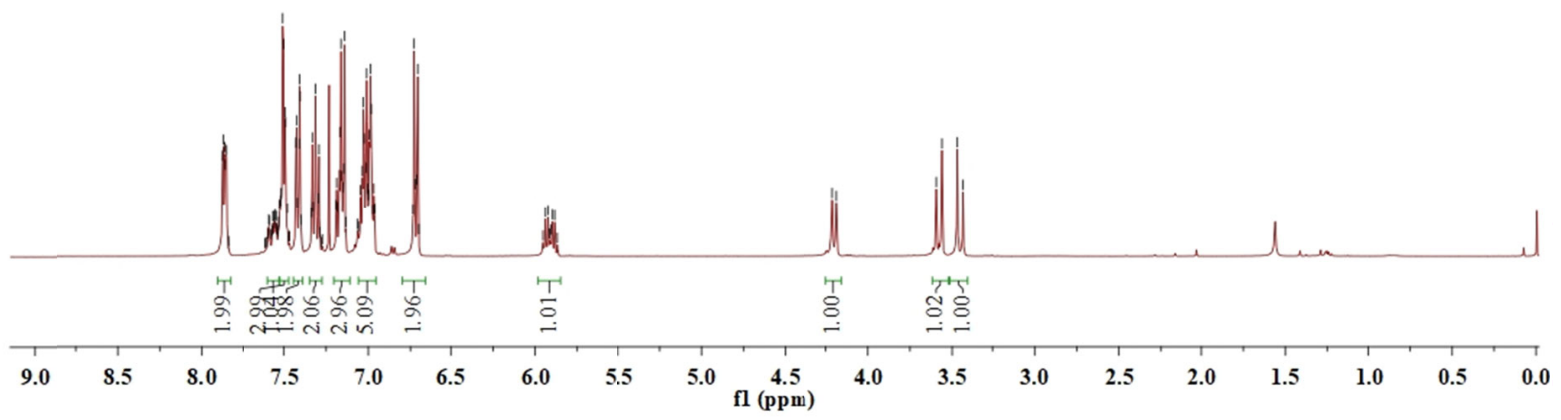

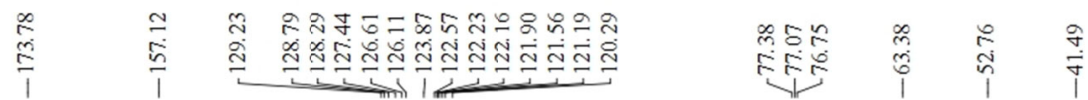

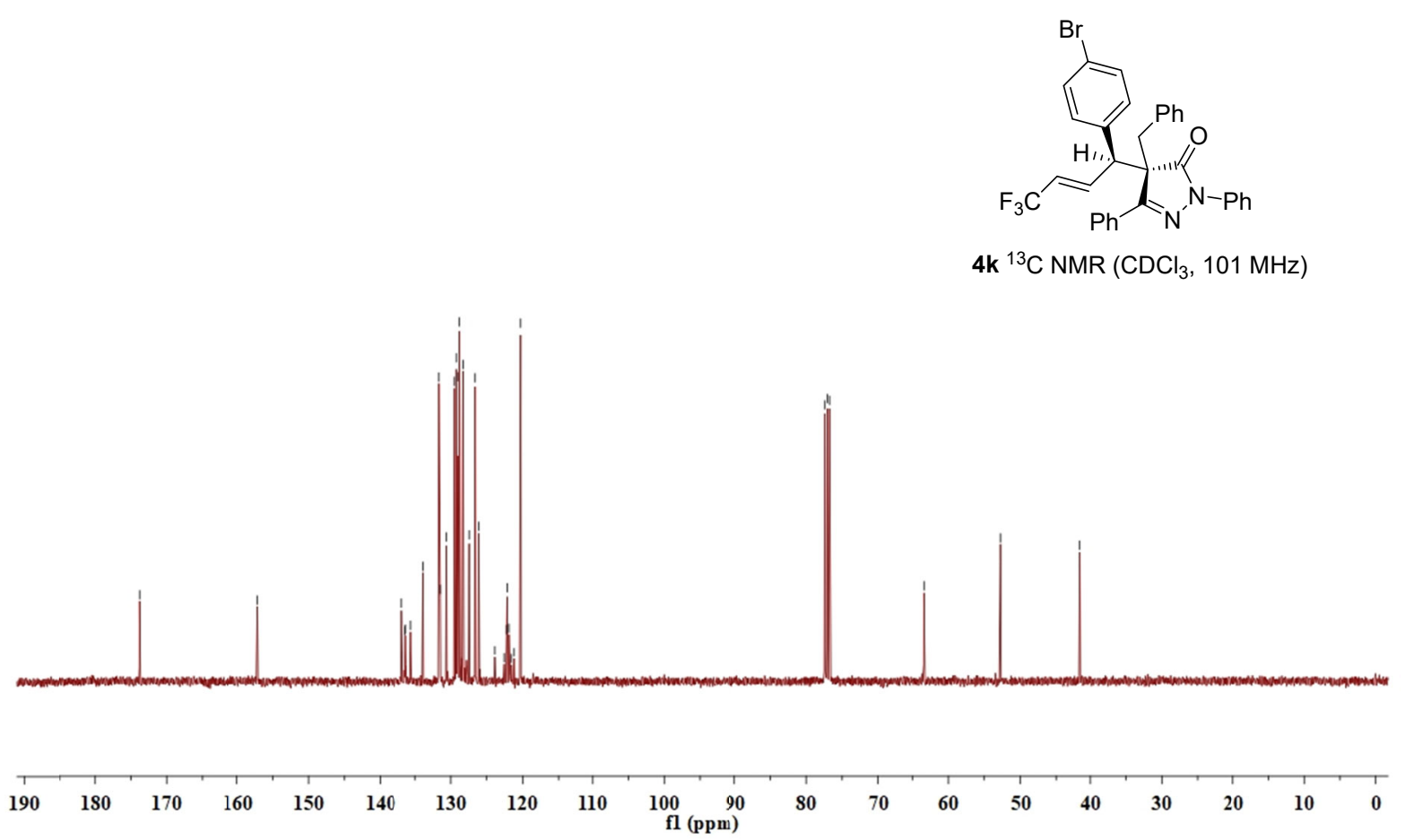




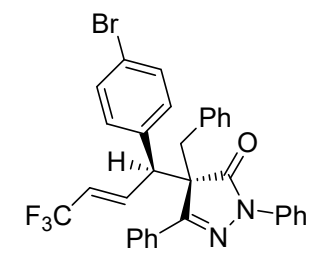

4k ${ }^{19} \mathrm{~F} \mathrm{NMR}\left(\mathrm{CDCl}_{3}, 377 \mathrm{MHz}\right)$
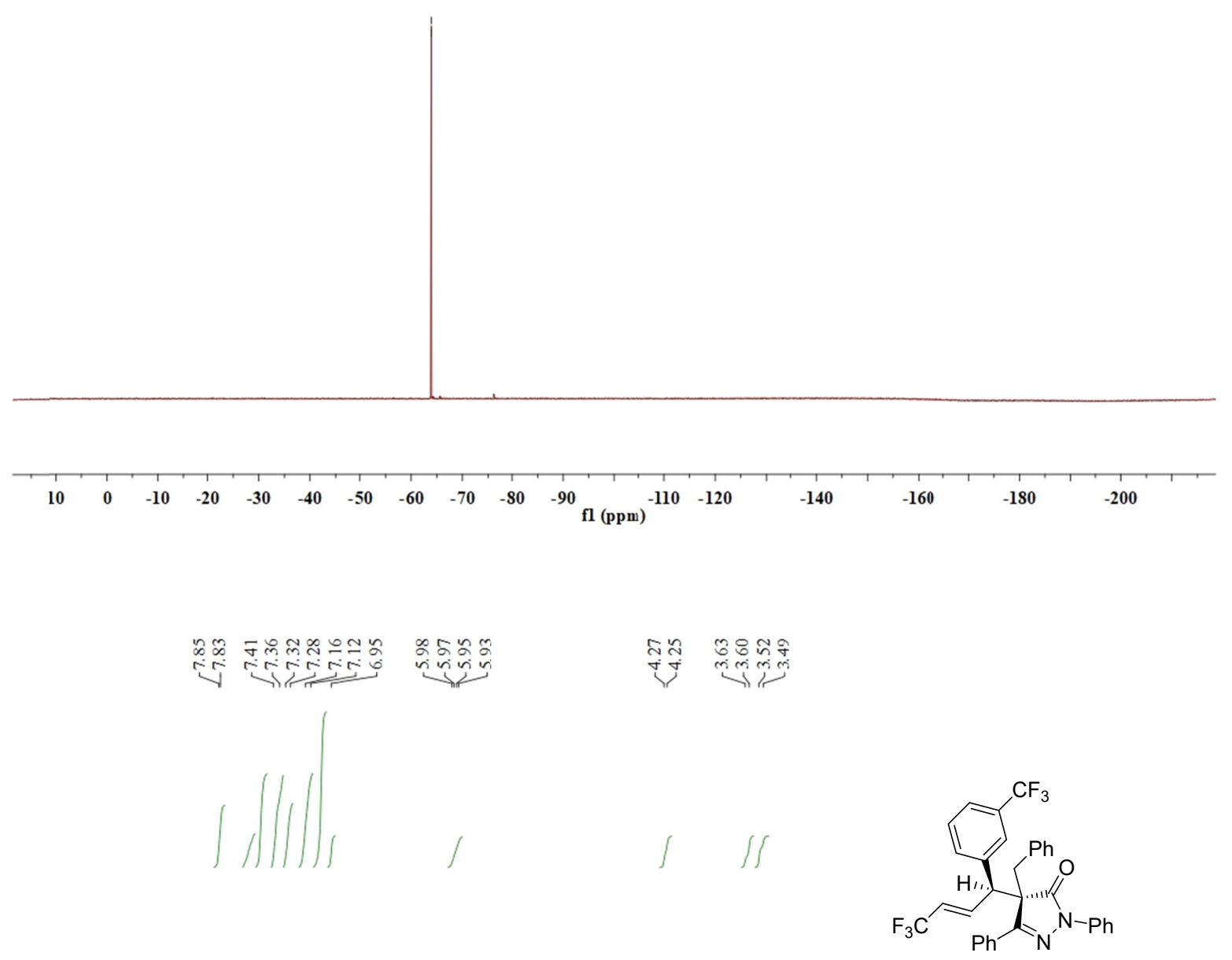

4I ${ }^{1} \mathrm{H}$ NMR $\left(\mathrm{CDCl}_{3}, 400 \mathrm{MHz}\right)$

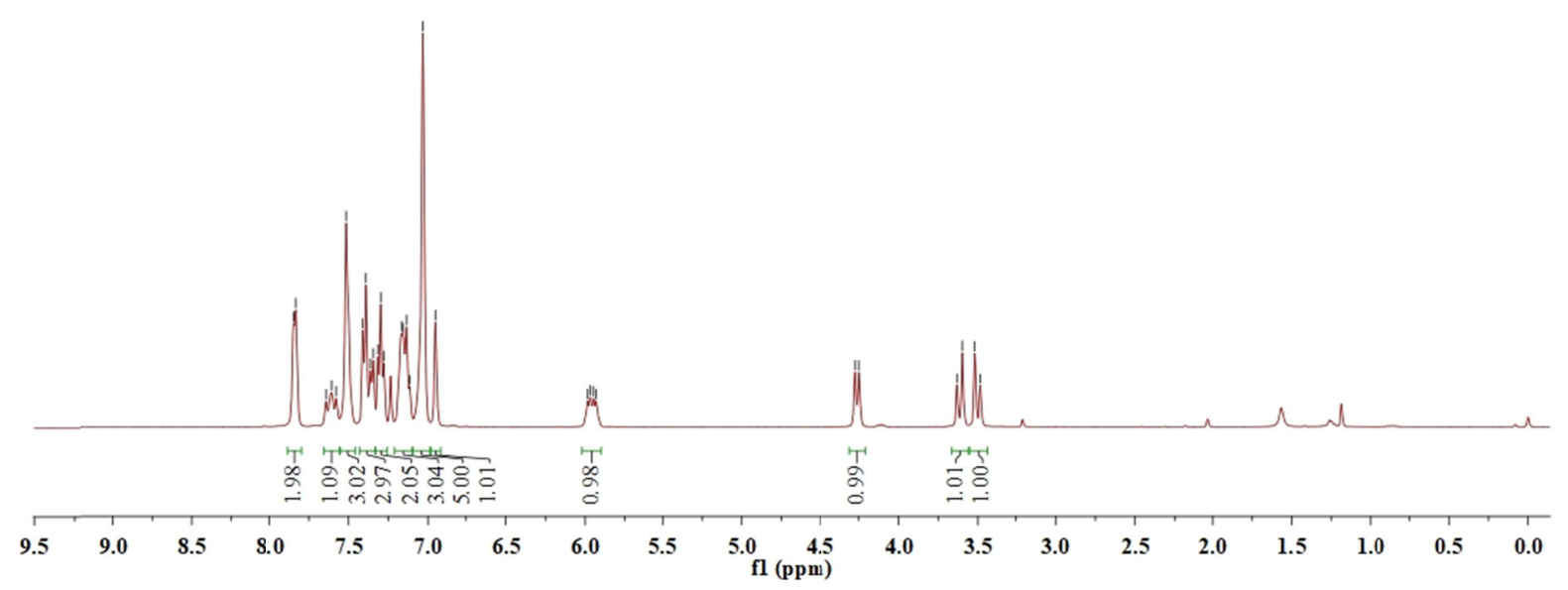




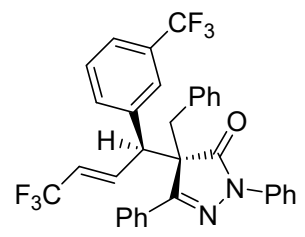

$4 \mathrm{I}^{13} \mathrm{C}$ NMR $\left(\mathrm{CDCl}_{3}, 101 \mathrm{MHz}\right)$
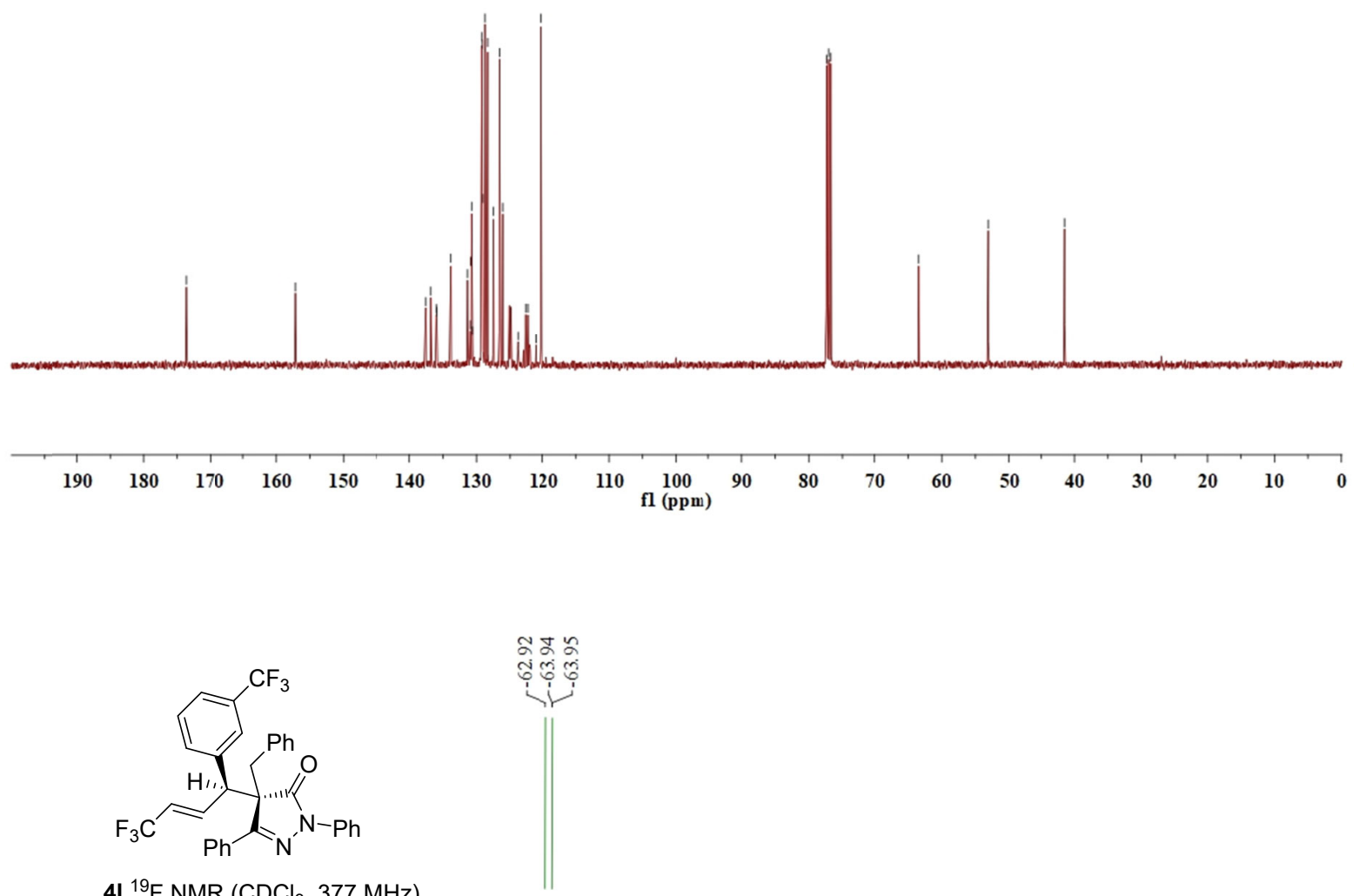

4I ${ }^{19} \mathrm{~F} \mathrm{NMR}\left(\mathrm{CDCl}_{3}, 377 \mathrm{MHz}\right)$

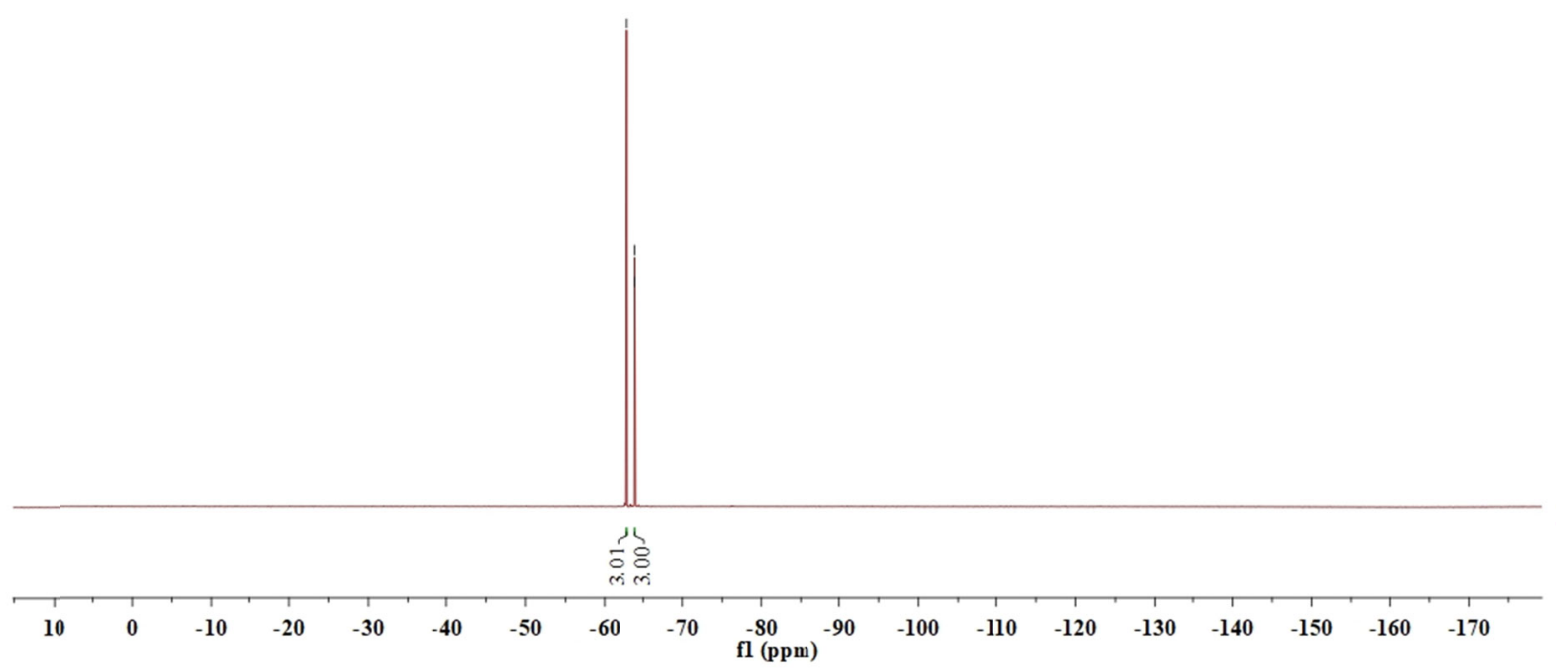




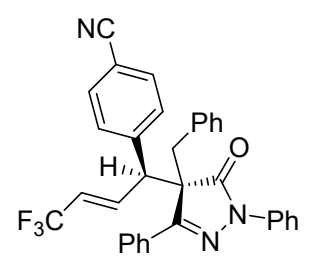

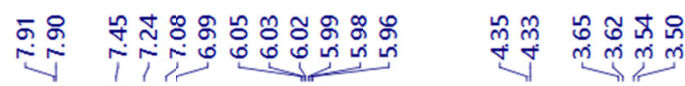

$4 \mathrm{~m}^{1} \mathrm{H}$ NMR $\left(\mathrm{CDCl}_{3}, 400 \mathrm{MHz}\right)$
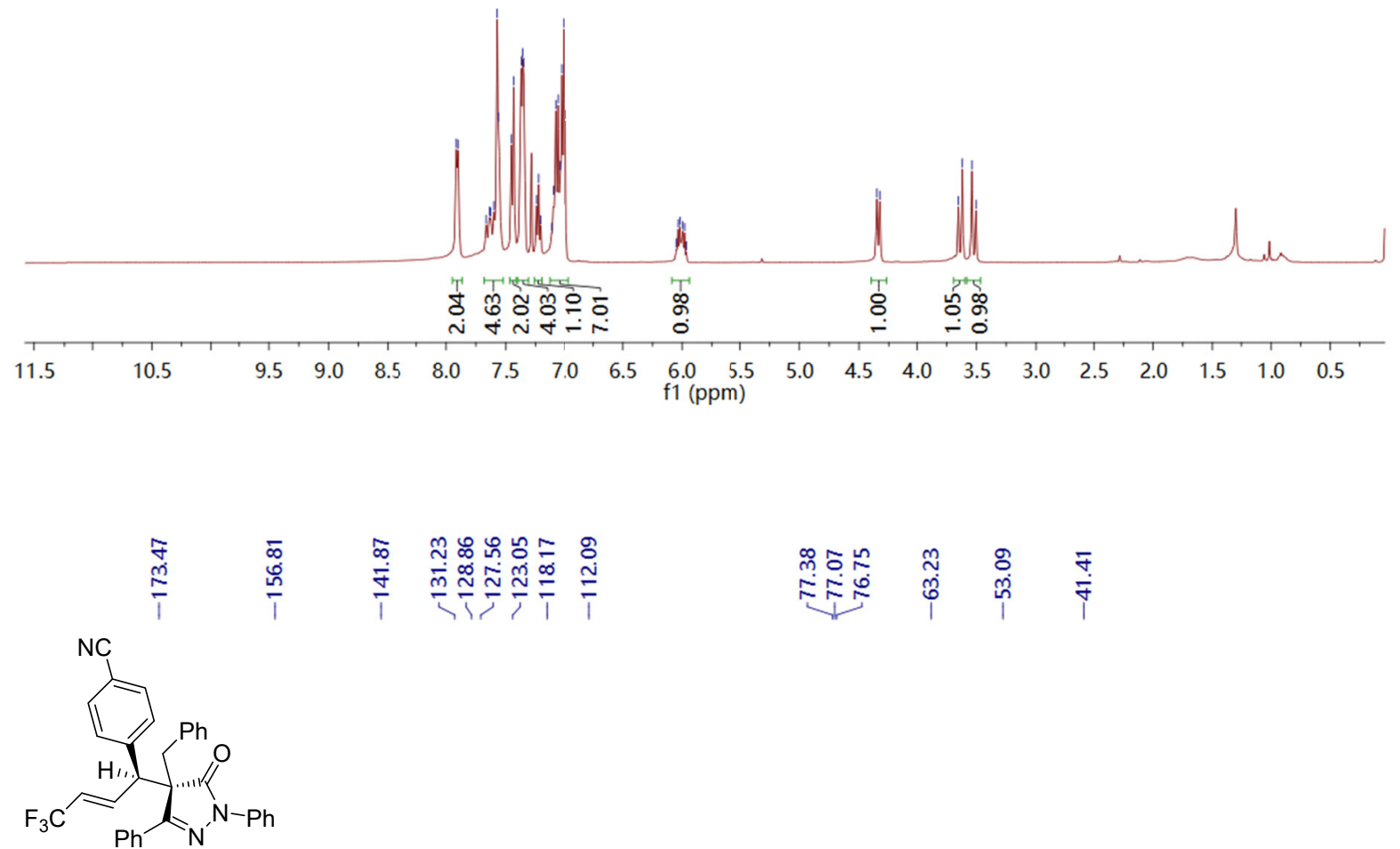

$4 \mathrm{~m}^{13} \mathrm{C}$ NMR $\left(\mathrm{CDCl}_{3}, 101 \mathrm{MHz}\right)$

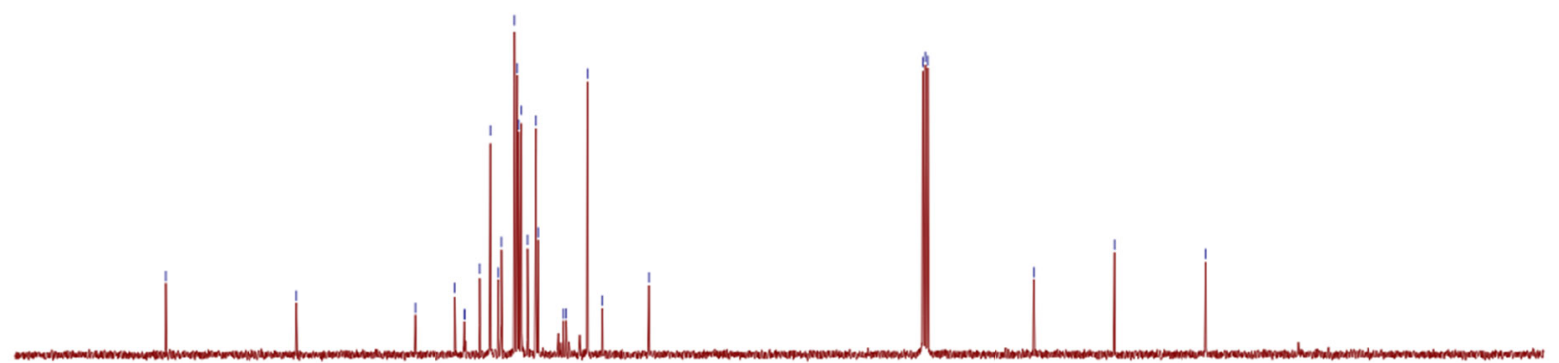

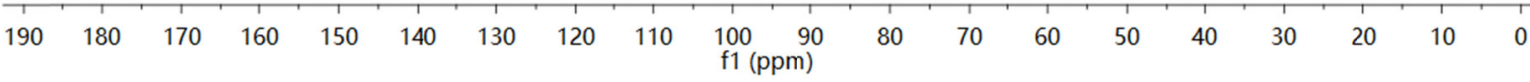




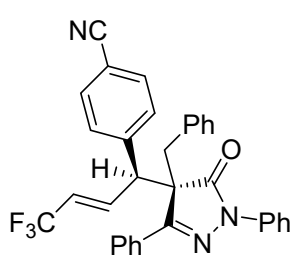

4m ${ }^{19} \mathrm{~F} \mathrm{NMR}\left(\mathrm{CDCl}_{3}, 377 \mathrm{MHz}\right)$
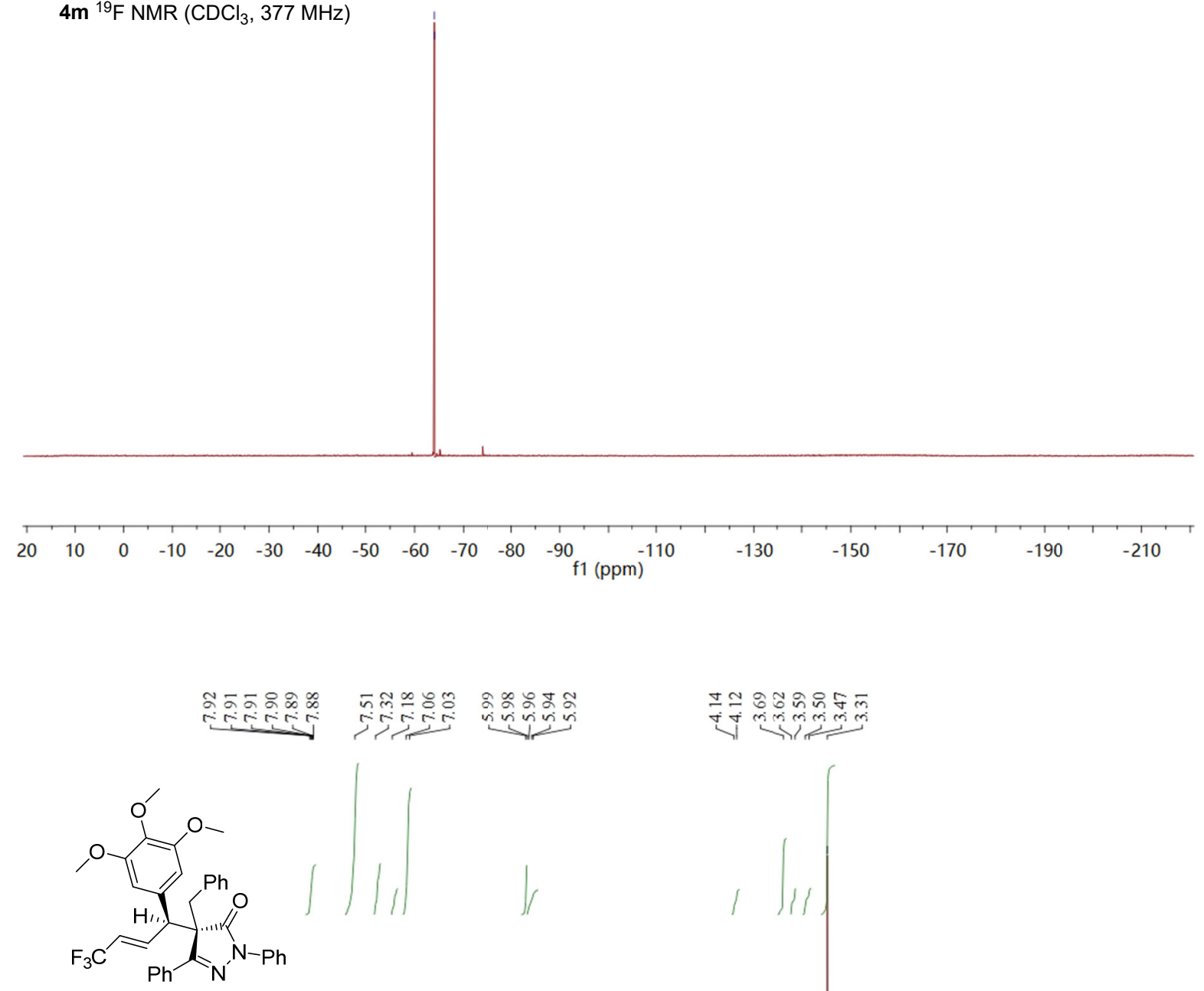

4n ${ }^{1} \mathrm{H}$ NMR $\left(\mathrm{CDCl}_{3}, 400 \mathrm{MHz}\right)$

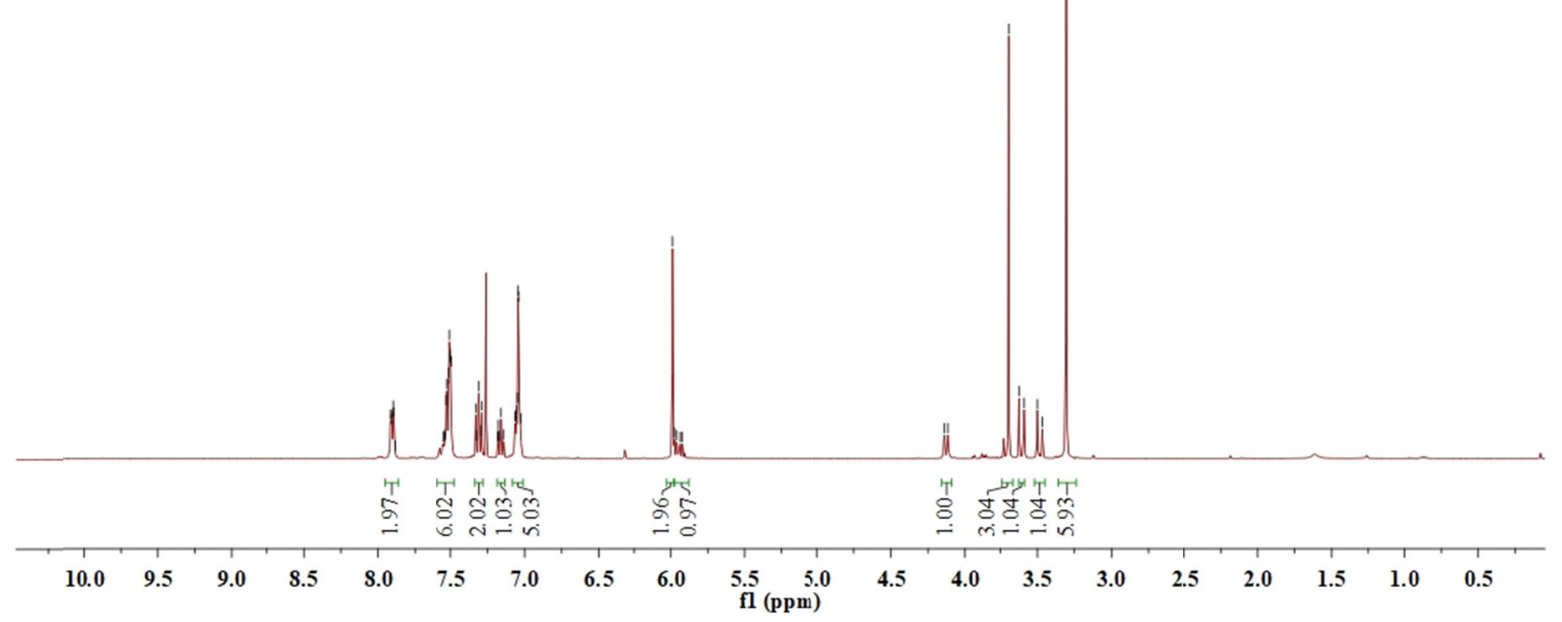




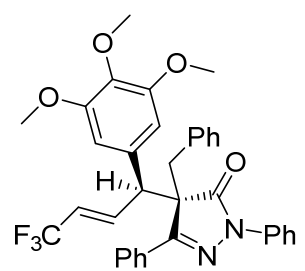

4n ${ }^{13} \mathrm{C} \mathrm{NMR}\left(\mathrm{CDCl}_{3}, 101 \mathrm{MHz}\right)$
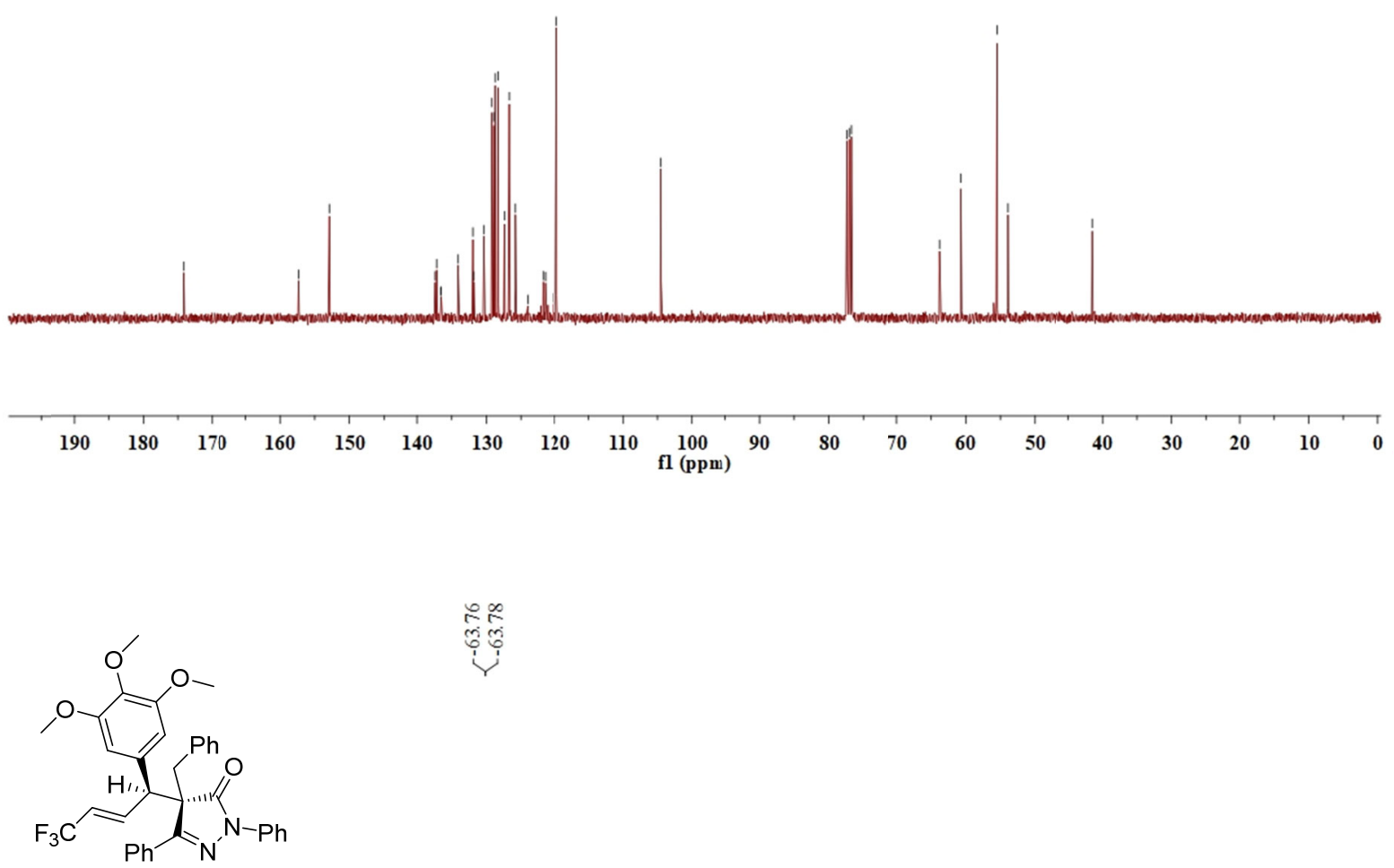

4n ${ }^{19} \mathrm{~F} \mathrm{NMR}\left(\mathrm{CDCl}_{3}, 377 \mathrm{MHz}\right)$

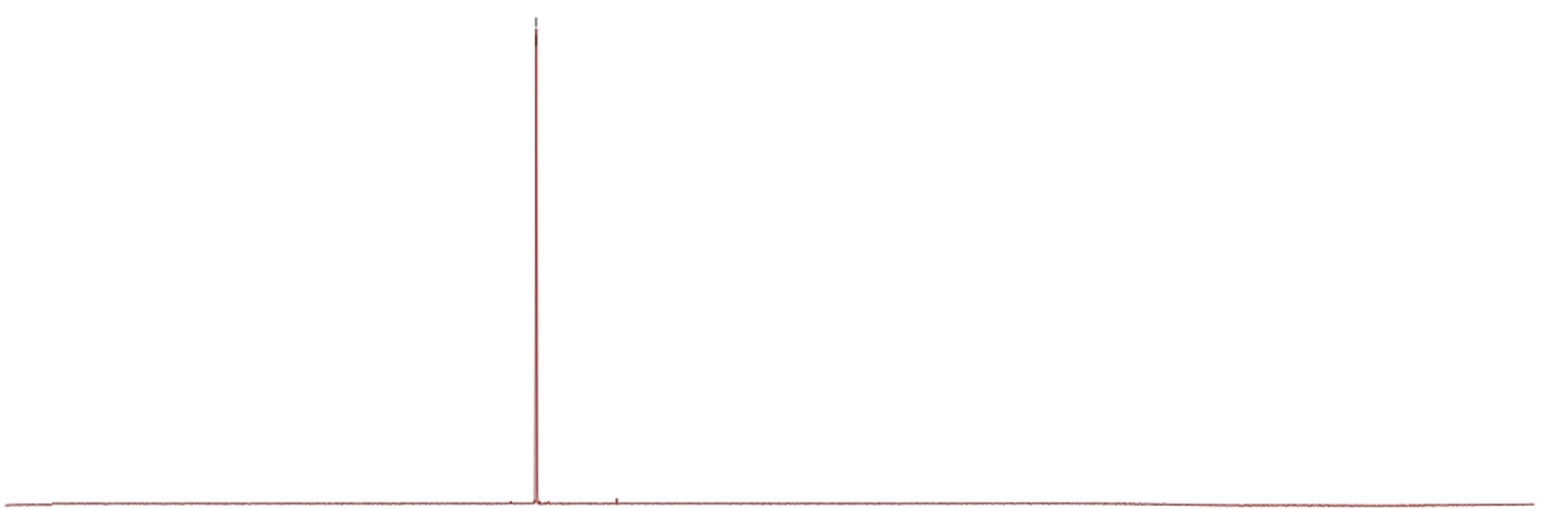

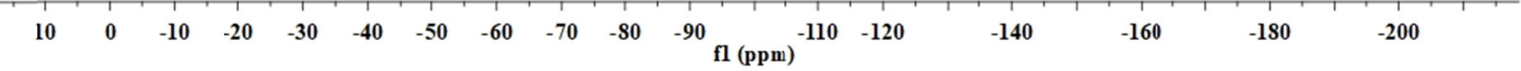




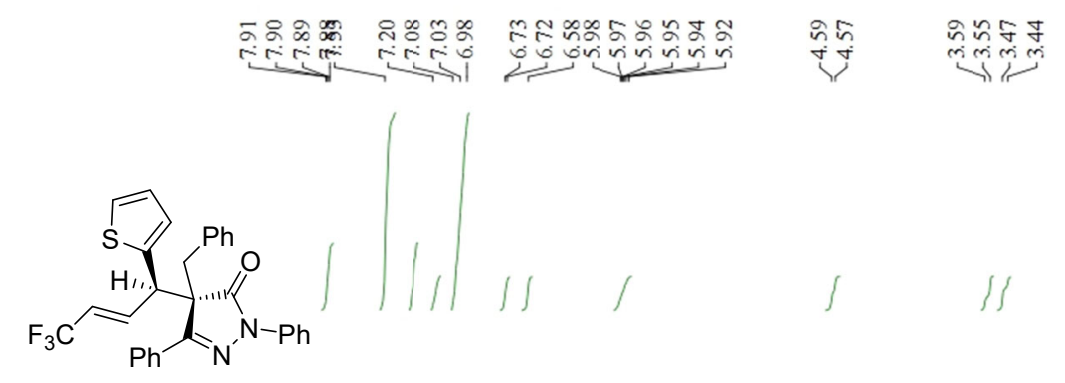

40 ${ }^{1} \mathrm{H}$ NMR $\left(\mathrm{CDCl}_{3}, 400 \mathrm{MHz}\right)$

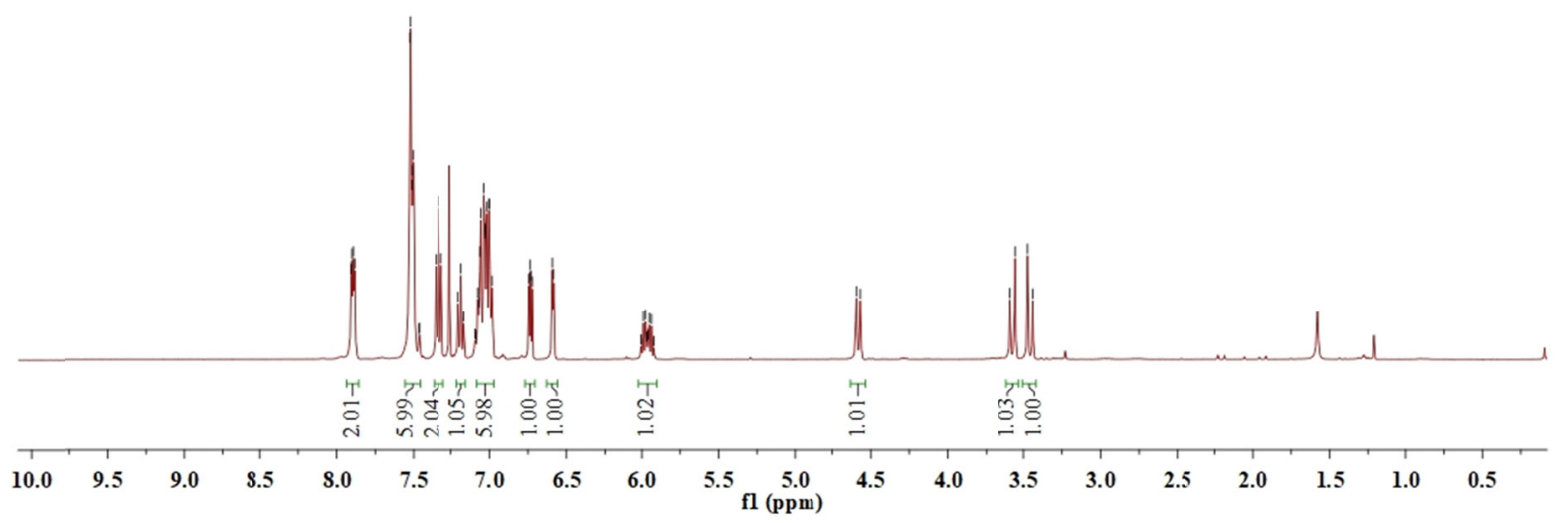

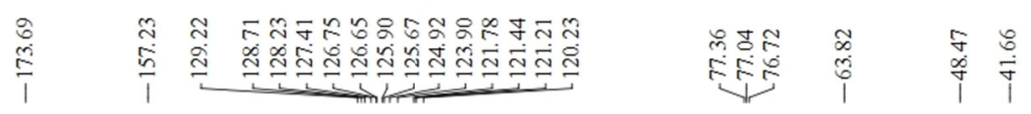

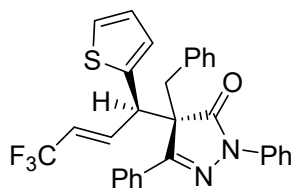

4o ${ }^{13} \mathrm{C} \mathrm{NMR}\left(\mathrm{CDCl}_{3}, 101 \mathrm{MHz}\right)$

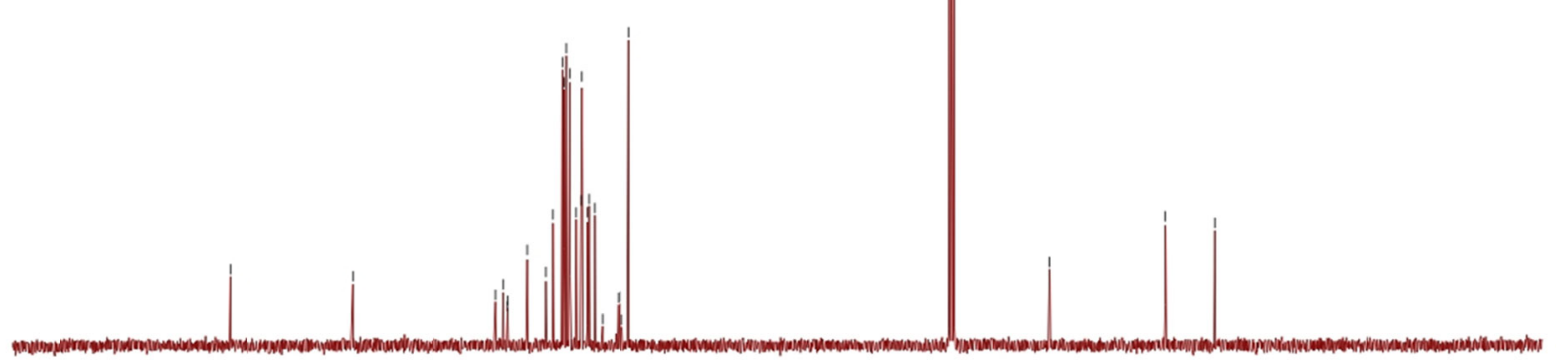

200

$\begin{array}{llllllll}190 & 180 & 170 & 160 & 150 & 140 & 130 & 120\end{array}$

$110 \underset{\mathrm{fl}(\mathrm{ppm})}{100} 90$

80

$60 \quad 50$

$\begin{array}{lllll}40 & 30 & 20 & 10 & 0\end{array}$ 


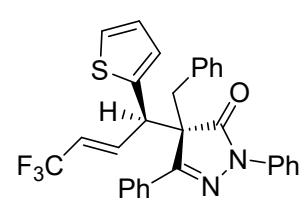

4o ${ }^{19} \mathrm{~F} \mathrm{NMR}\left(\mathrm{CDCl}_{3}, 377 \mathrm{MHz}\right)$
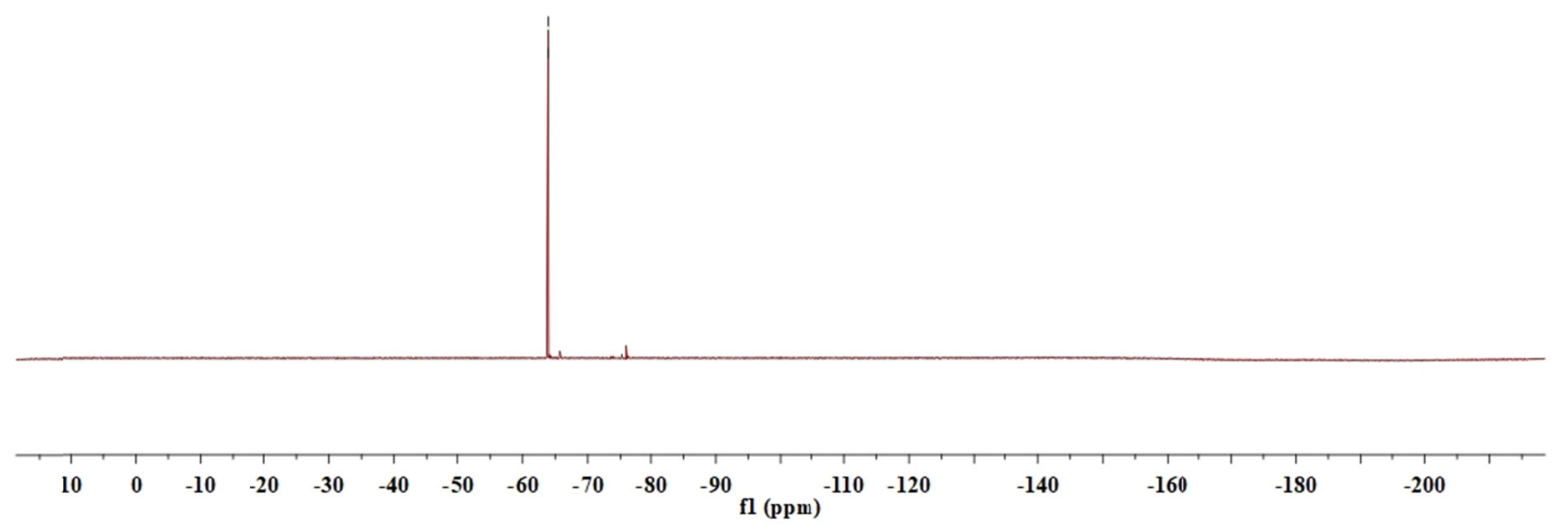

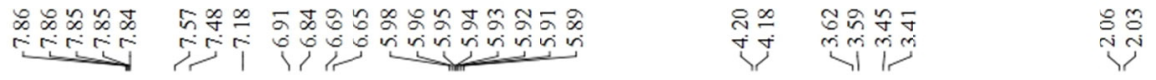
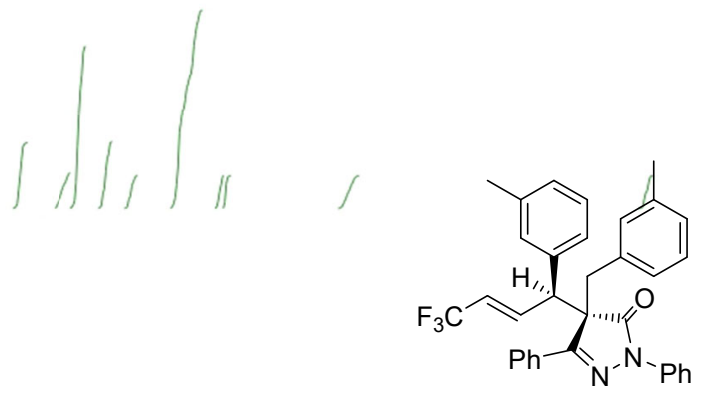

4q ${ }^{1} \mathrm{H} \mathrm{NMR}\left(\mathrm{CDCl}_{3}, 400 \mathrm{MHz}\right)$

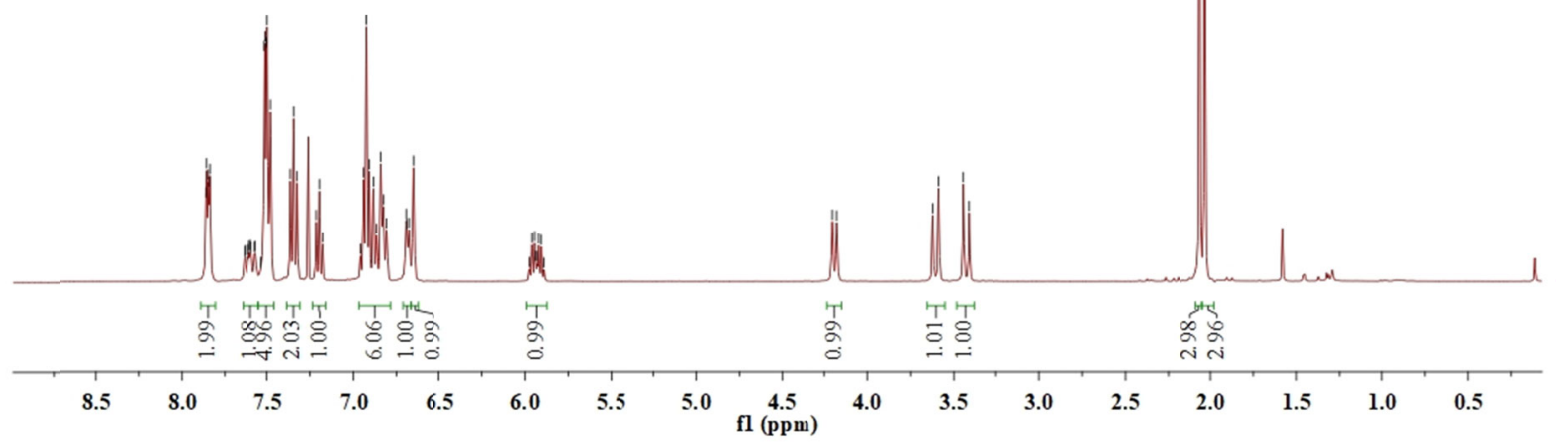




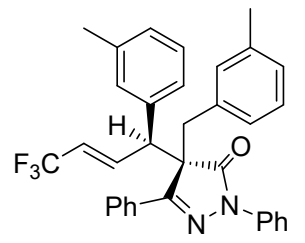

$4{ }^{13} \mathrm{C} \mathrm{NMR}\left(\mathrm{CDCl}_{3}, 101 \mathrm{MHz}\right)$
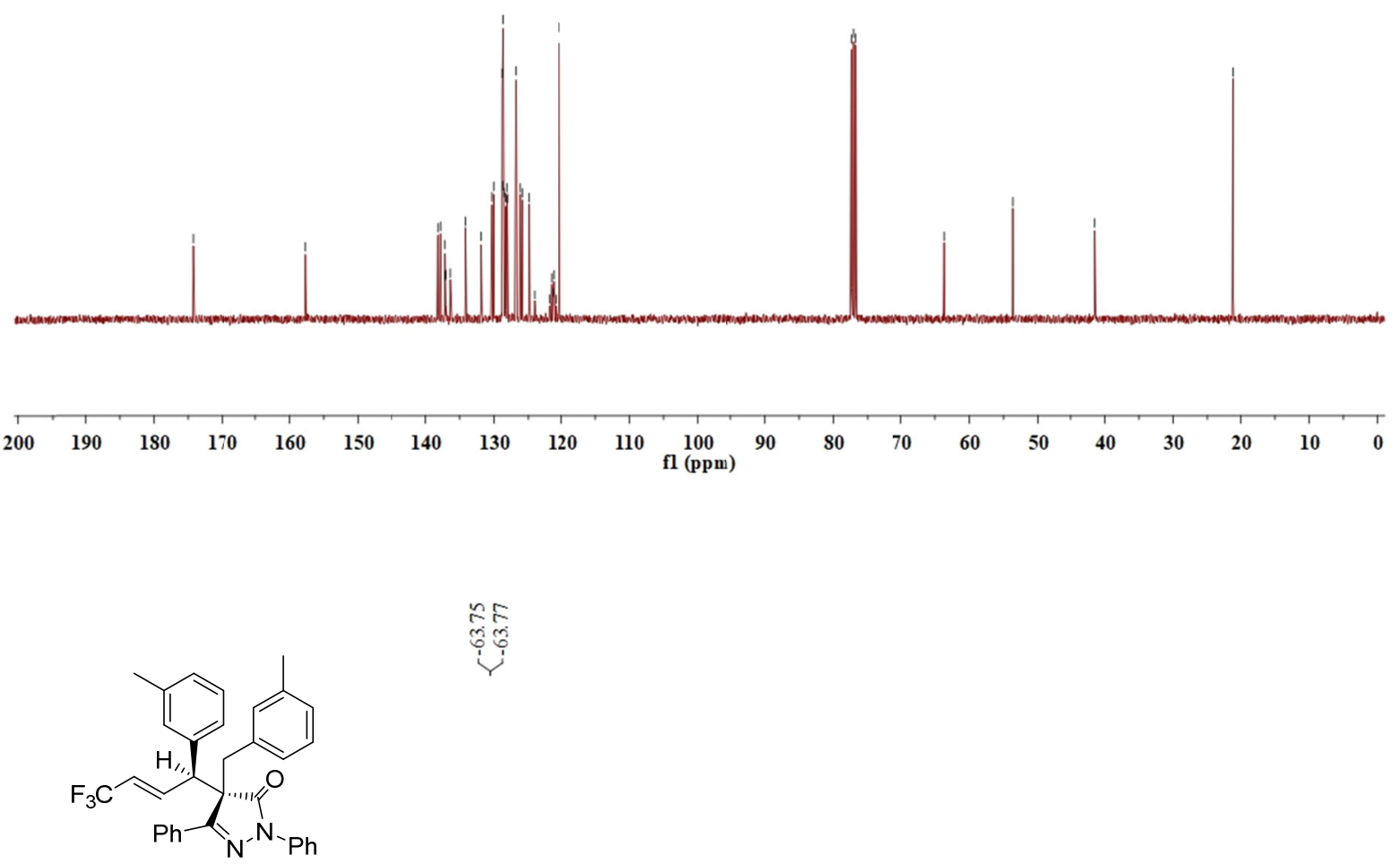

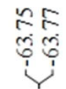

$4 \mathbf{q}^{19} \mathrm{~F} \mathrm{NMR}\left(\mathrm{CDCl}_{3}, 377 \mathrm{MHz}\right)$

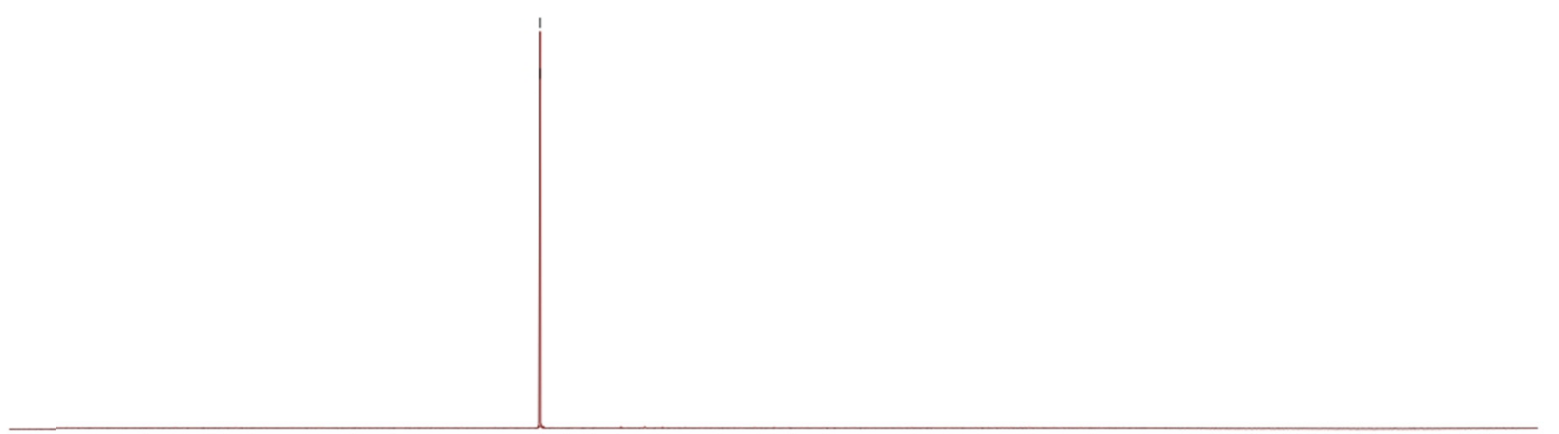

$\begin{array}{llllllllllllllllllllllllll}10 & 0 & -10 & -20 & -30 & -40 & -50 & -60 & -70 & -80 & -90 & & -110 & -120 & -140 & -160 & -180 & -200 & \end{array}$ 


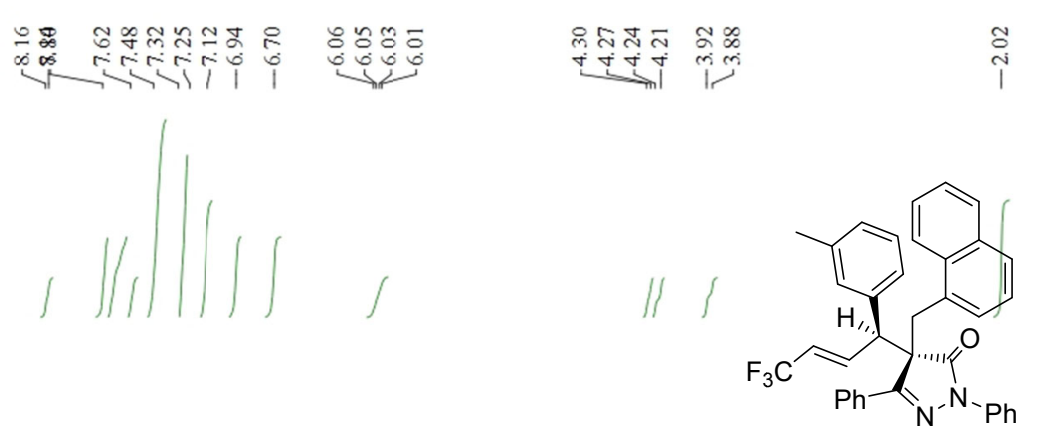

$4 \mathbf{r}^{1} \mathrm{H} \mathrm{NMR}\left(\mathrm{CDCl}_{3}, 400 \mathrm{MHz}\right)$

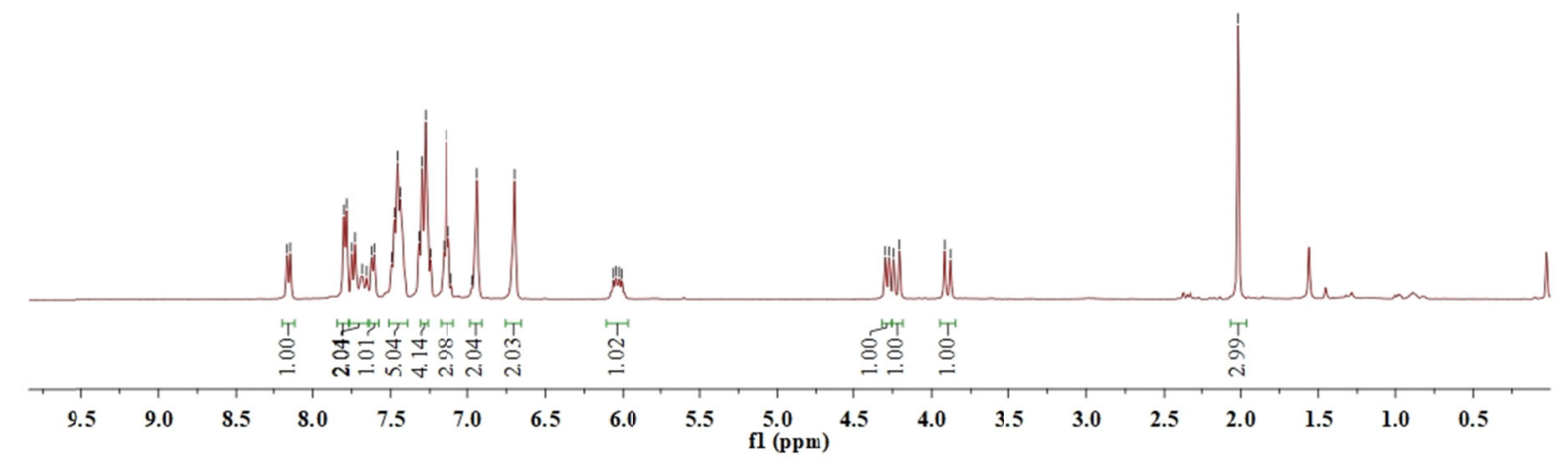

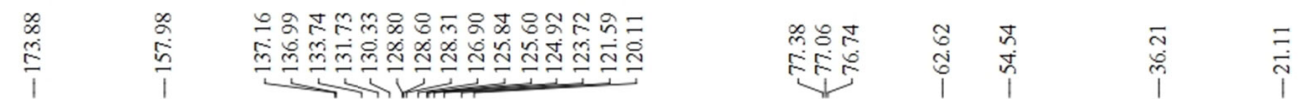

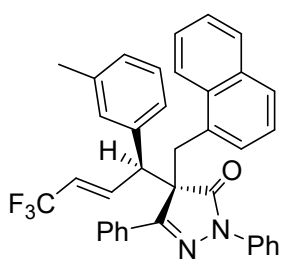

$4 \mathbf{r}^{13} \mathrm{C} \mathrm{NMR}\left(\mathrm{CDCl}_{3}, 101 \mathrm{MHz}\right)$

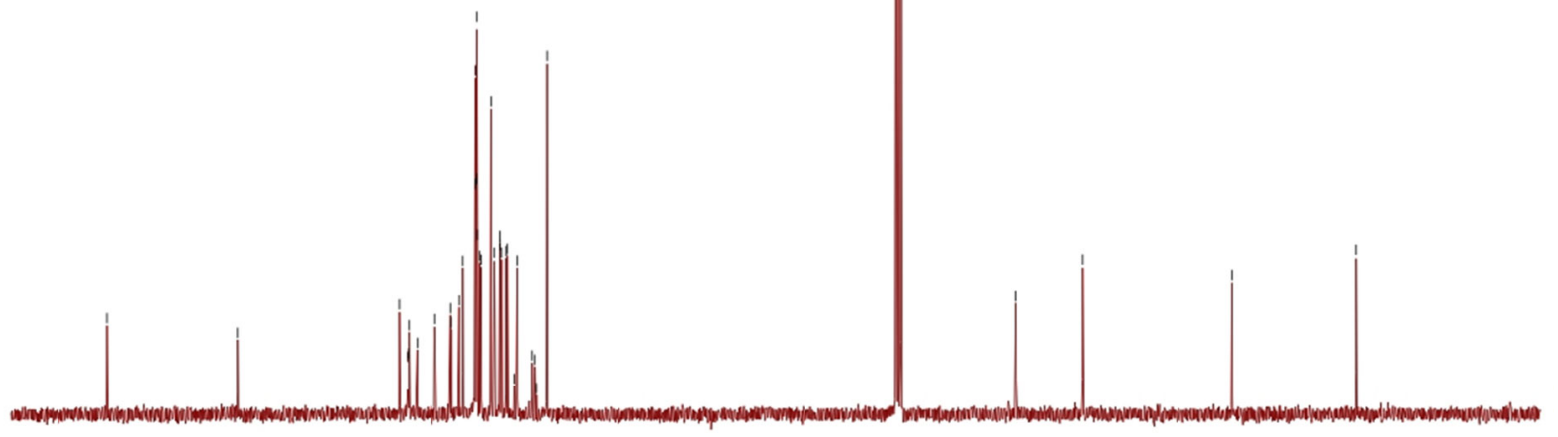

$190 \quad 170$ 120 $100 \underset{\mathrm{fl}(\mathrm{ppm})}{90} \mathrm{~s}$ 60 50 $\begin{array}{lllll}1 & 30 & 20 & 10 & 0\end{array}$ 


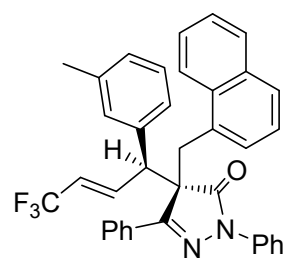

$4 \mathbf{r}^{19} \mathrm{~F} \mathrm{NMR}\left(\mathrm{CDCl}_{3}, 377 \mathrm{MHz}\right)$

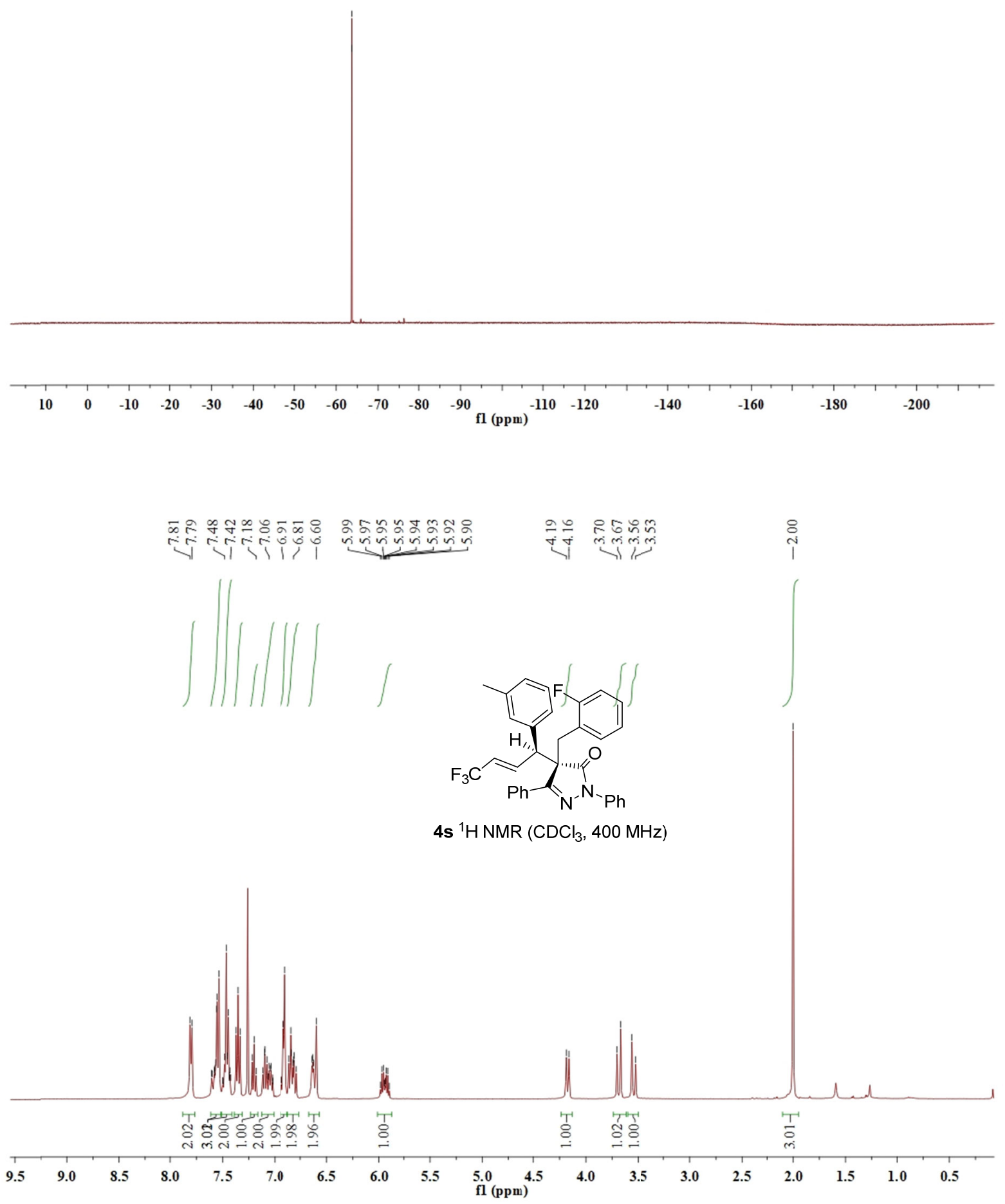



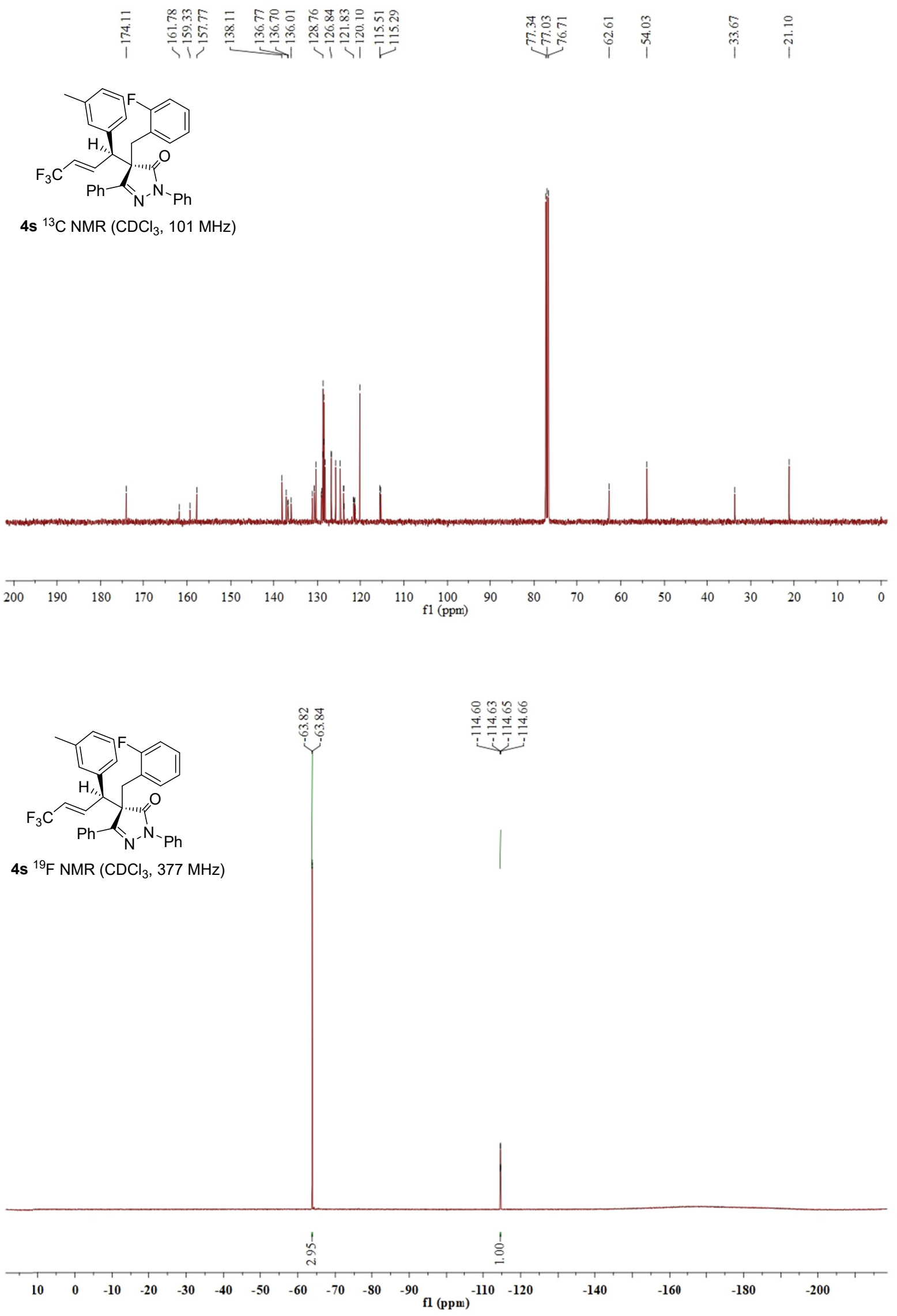

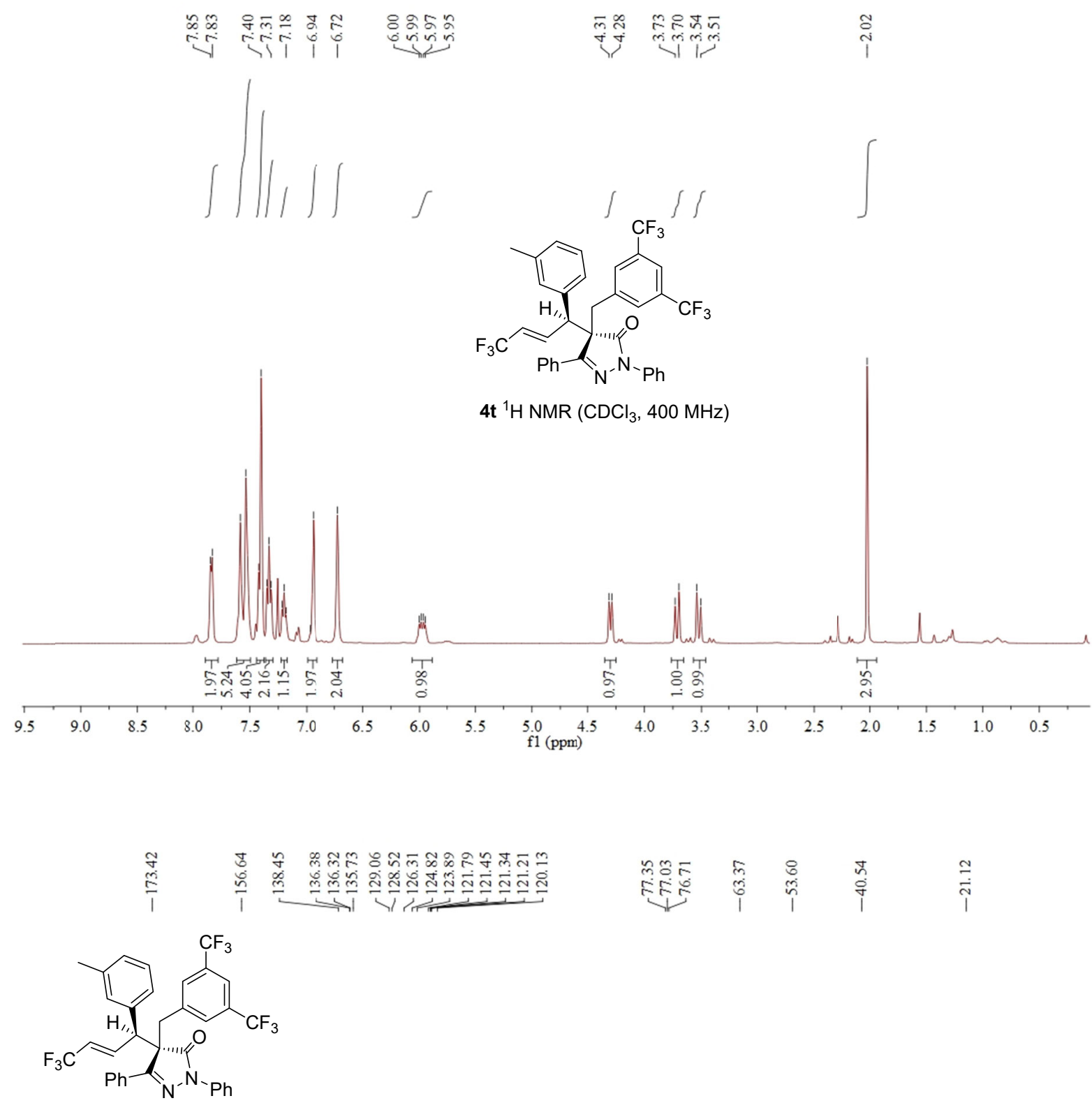

4t ${ }^{13} \mathrm{C} \mathrm{NMR}\left(\mathrm{CDCl}_{3}, 101 \mathrm{MHz}\right)$

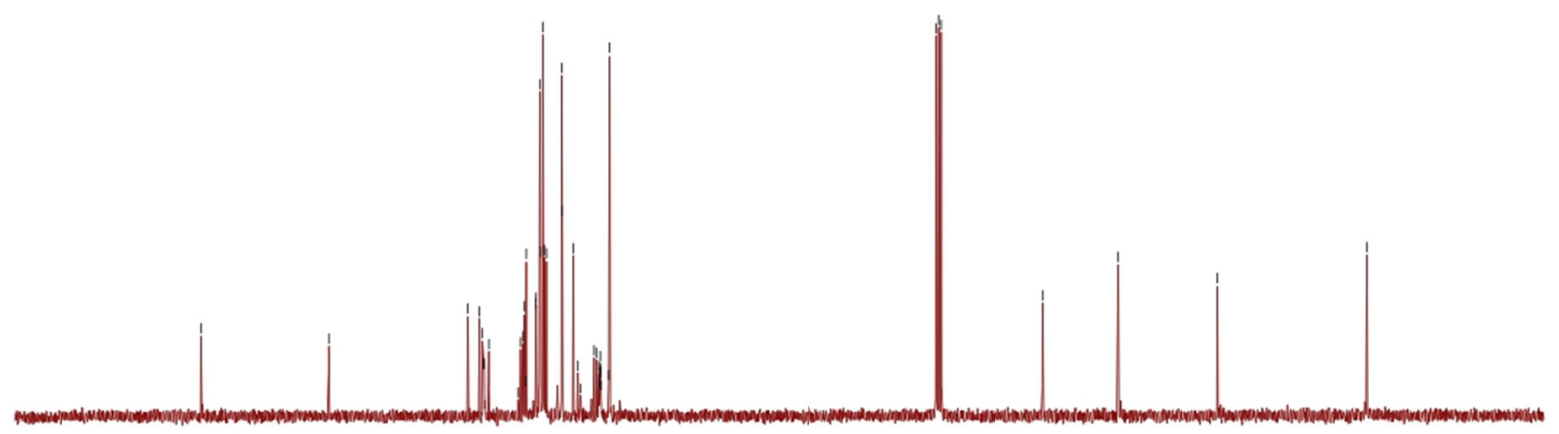

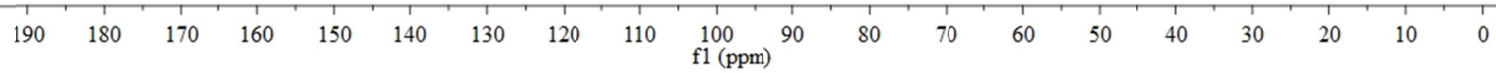




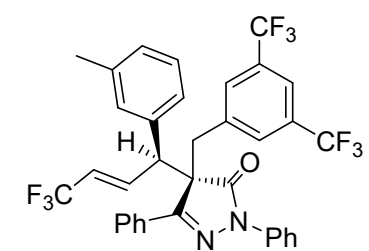

4t ${ }^{19} \mathrm{~F} \mathrm{NMR}\left(\mathrm{CDCl}_{3}, 377 \mathrm{MHz}\right)$

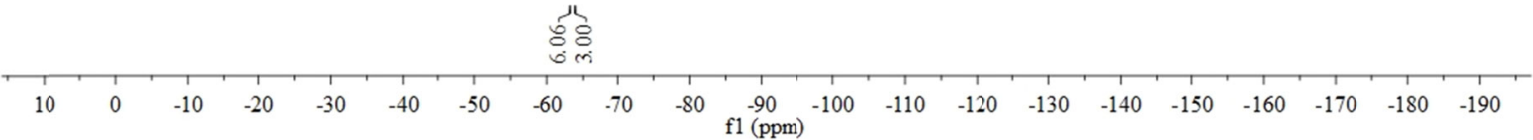

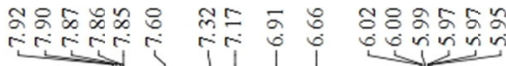

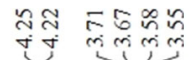

$\stackrel{\bar{\sigma}}{i}$
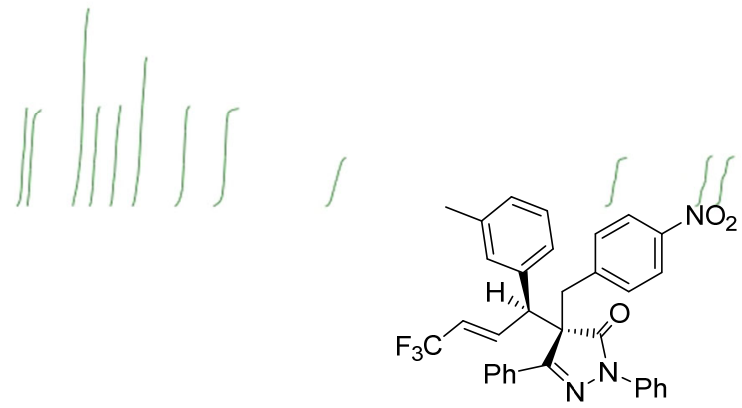

4u ${ }^{1} \mathrm{H}$ NMR $\left(\mathrm{CDCl}_{3}, 400 \mathrm{MHz}\right)$

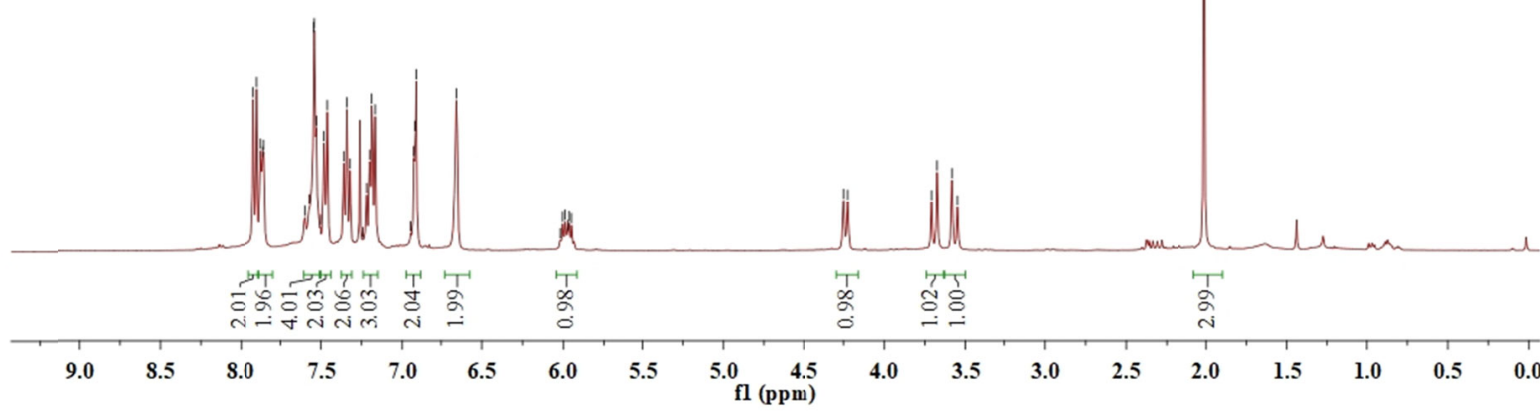




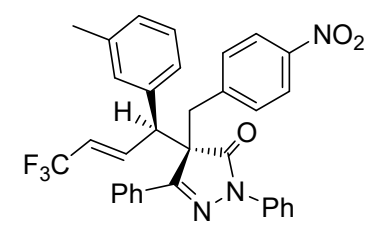

$4 \mathrm{u}^{13} \mathrm{C}$ NMR $\left(\mathrm{CDCl}_{3}, 101 \mathrm{MHz}\right)$
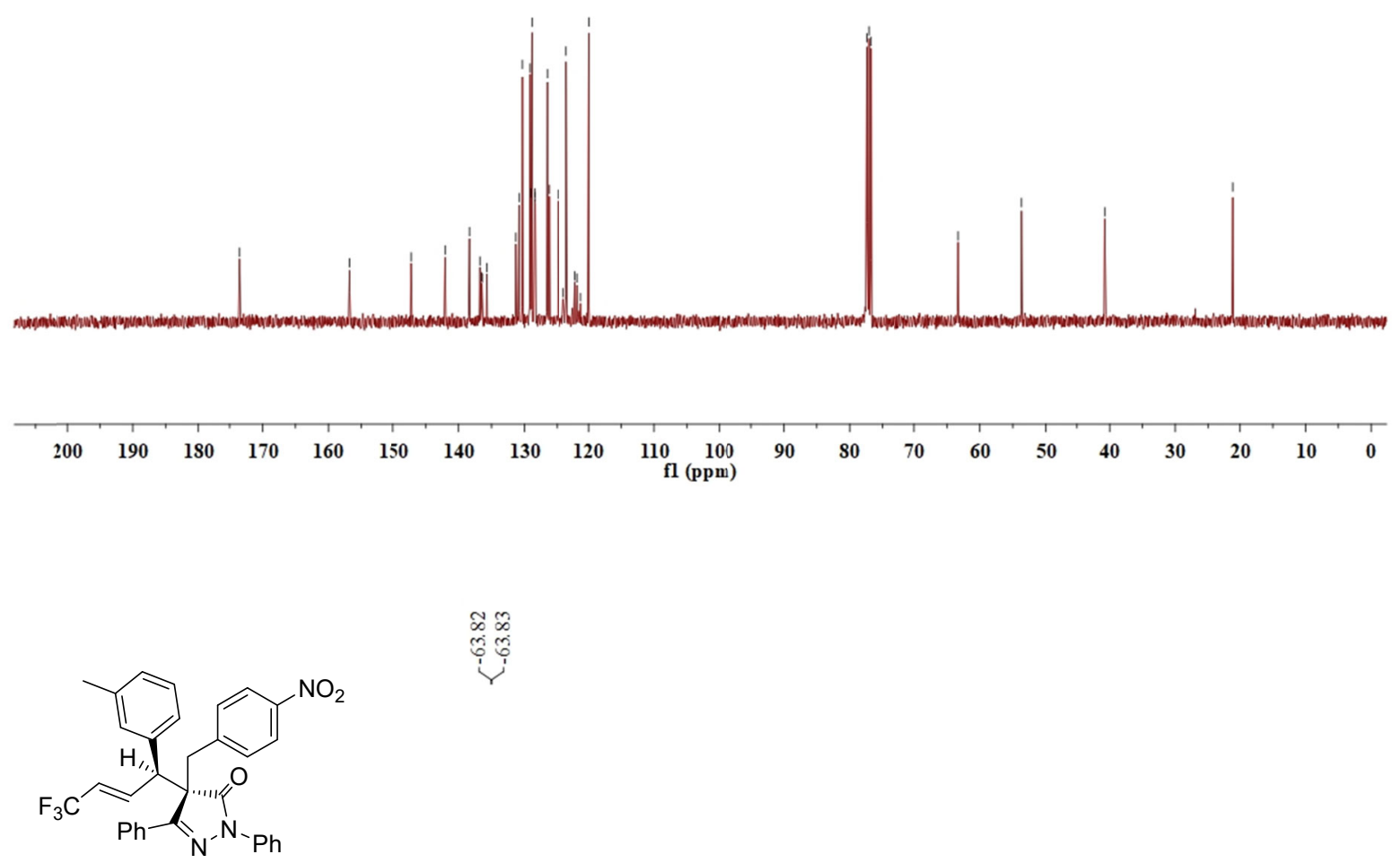

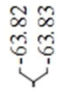

4u ${ }^{19} \mathrm{~F} \mathrm{NMR}\left(\mathrm{CDCl}_{3}, 377 \mathrm{MHz}\right)$

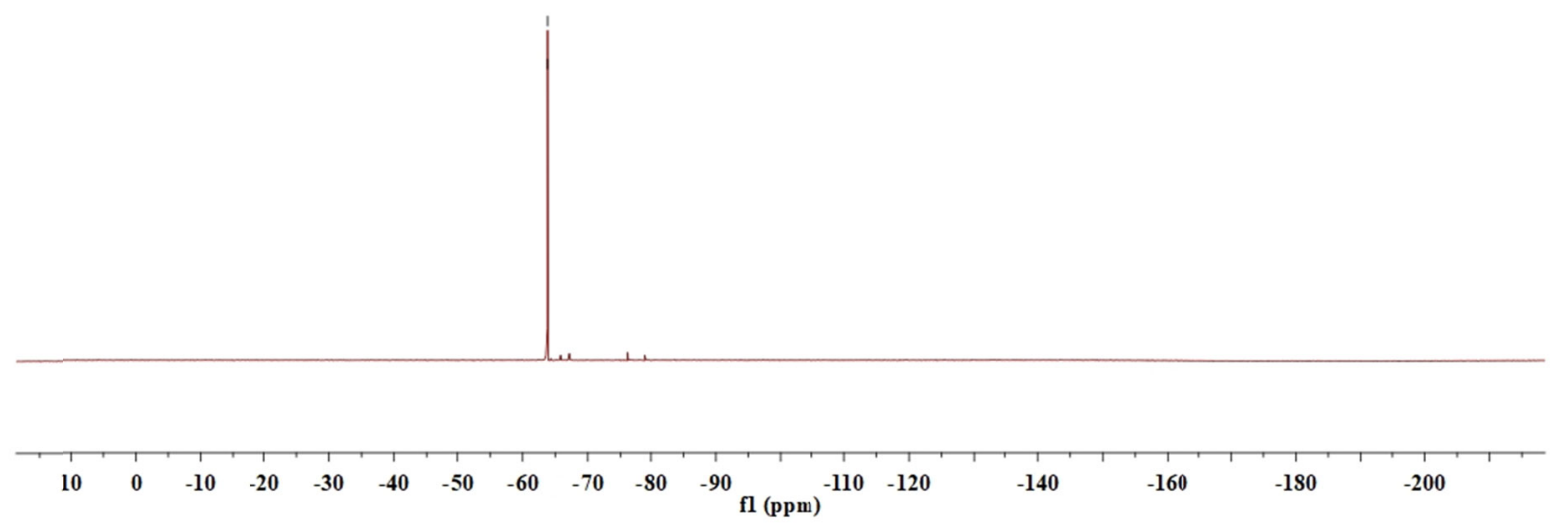




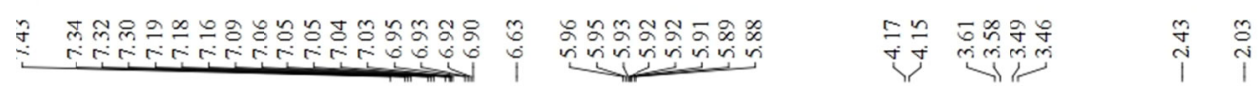
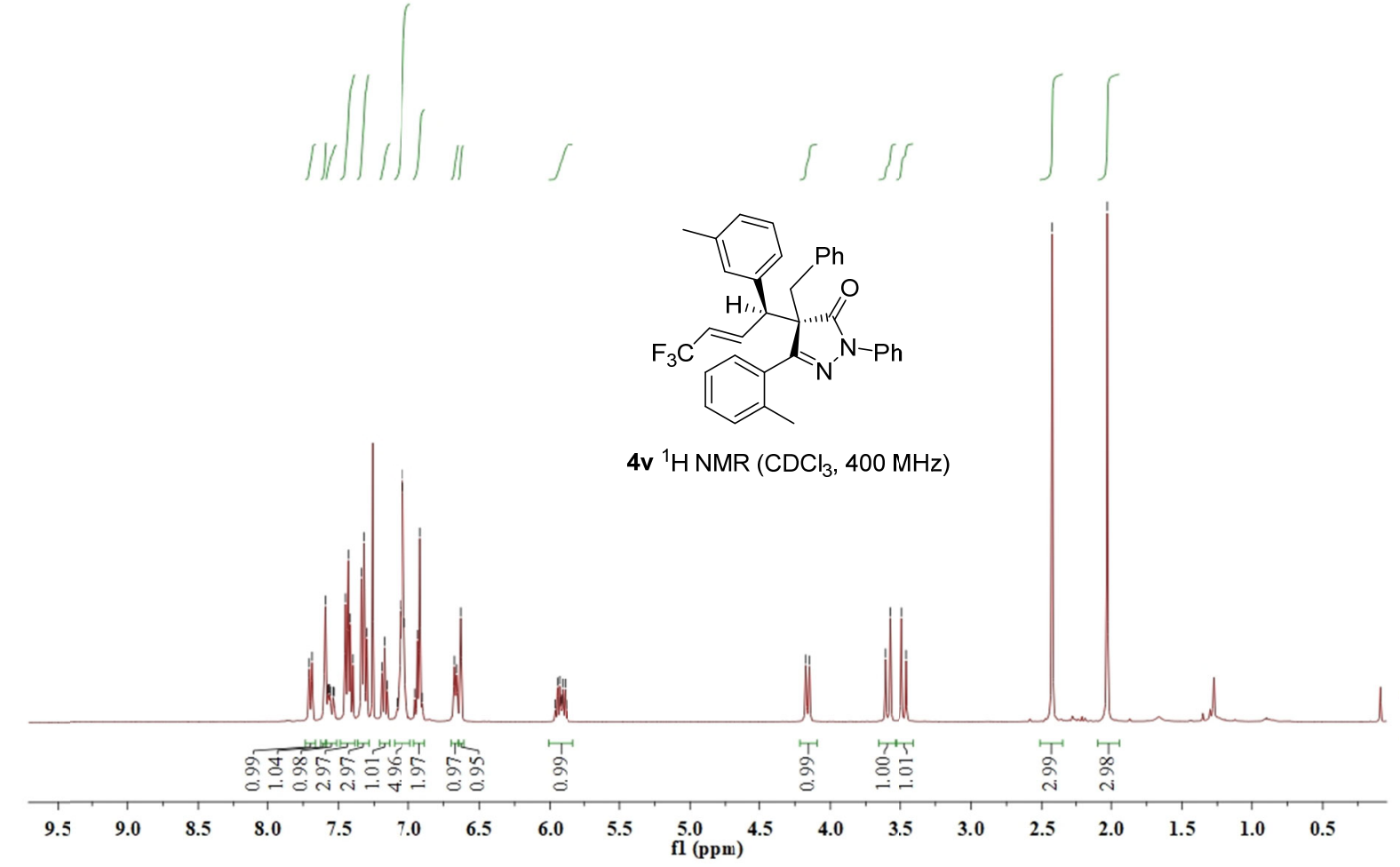

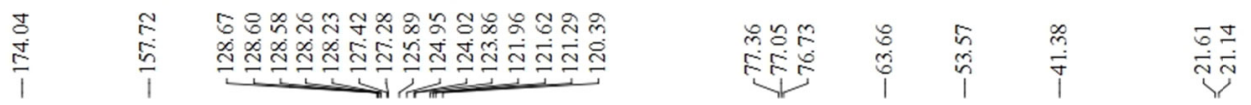

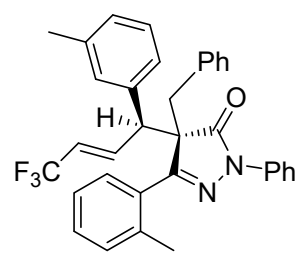

4v ${ }^{13} \mathrm{C}$ NMR $\left(\mathrm{CDCl}_{3}, 101 \mathrm{MHz}\right)$

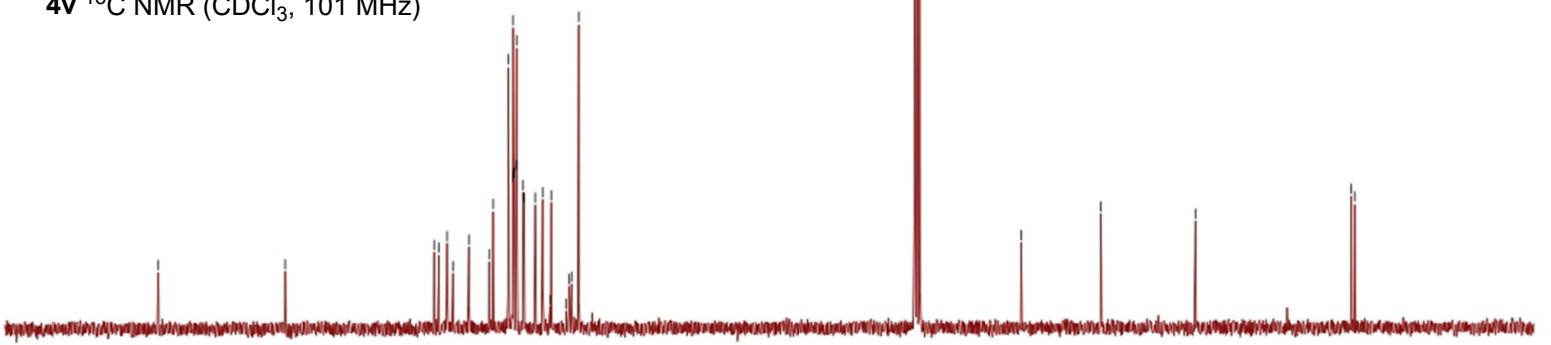




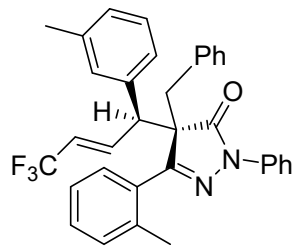

4v ${ }^{19} \mathrm{~F} \mathrm{NMR}\left(\mathrm{CDCl}_{3}, 377 \mathrm{MHz}\right)$

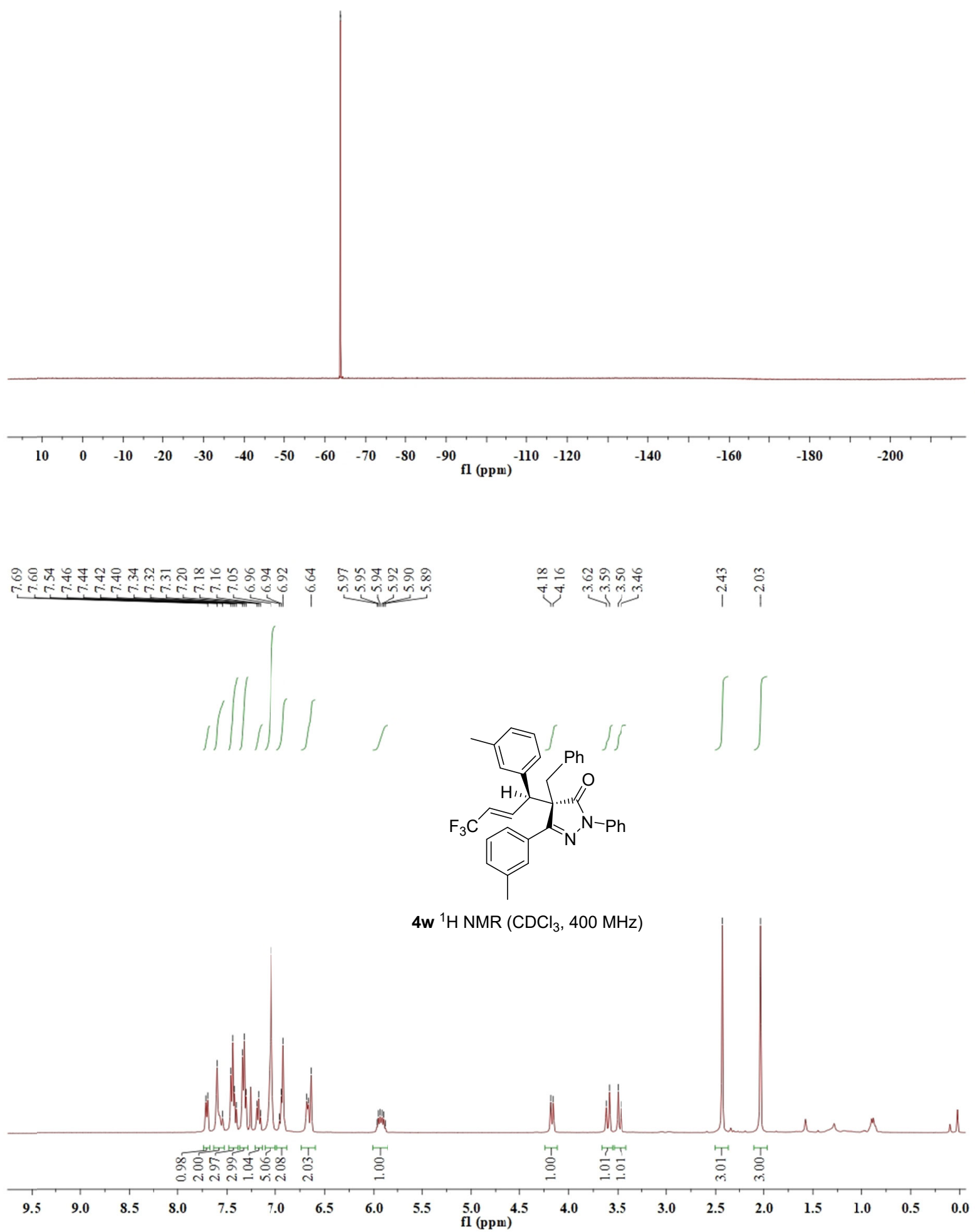




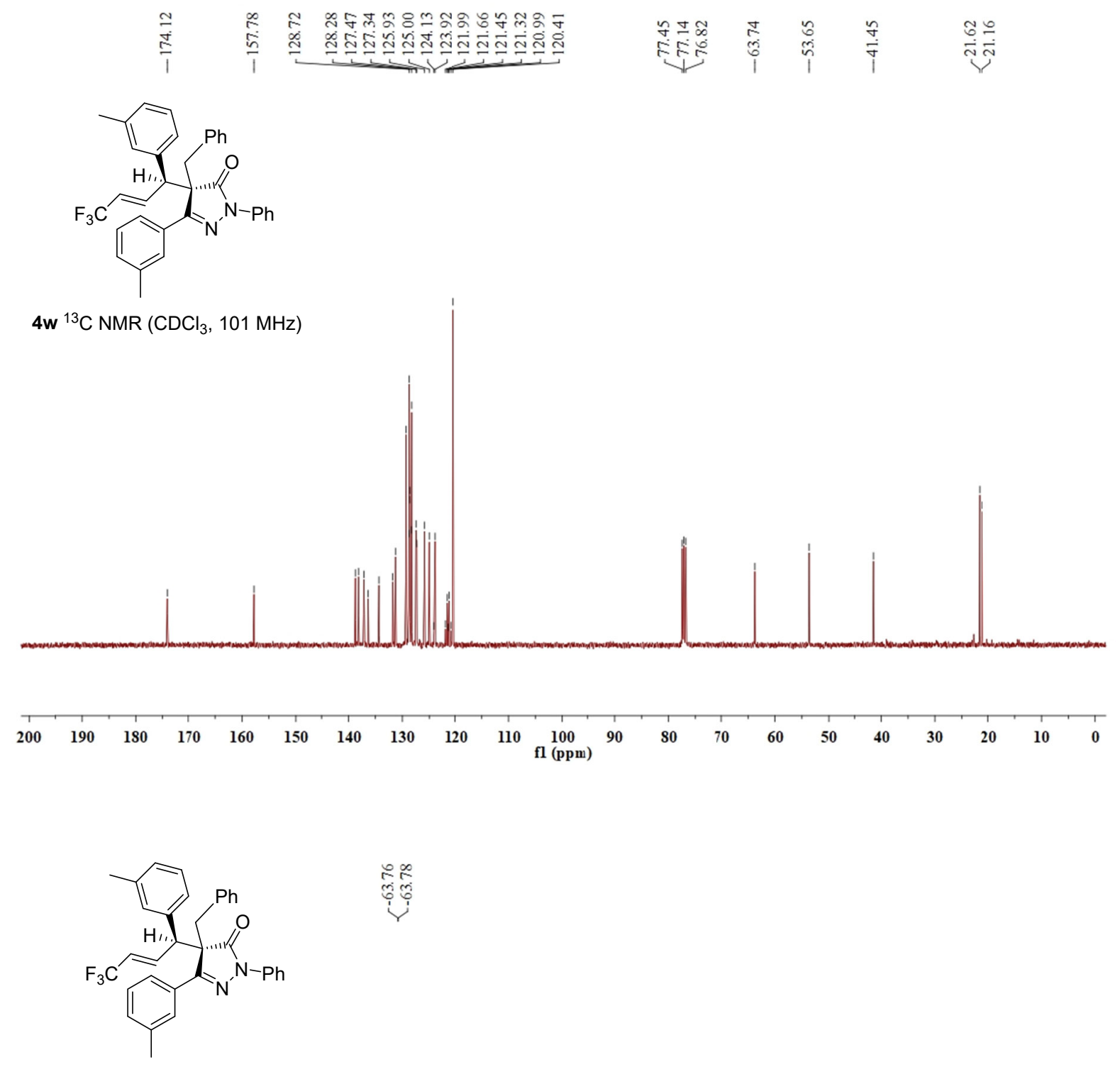

4w ${ }^{19} \mathrm{~F} \mathrm{NMR}\left(\mathrm{CDCl}_{3}, 377 \mathrm{MHz}\right)$

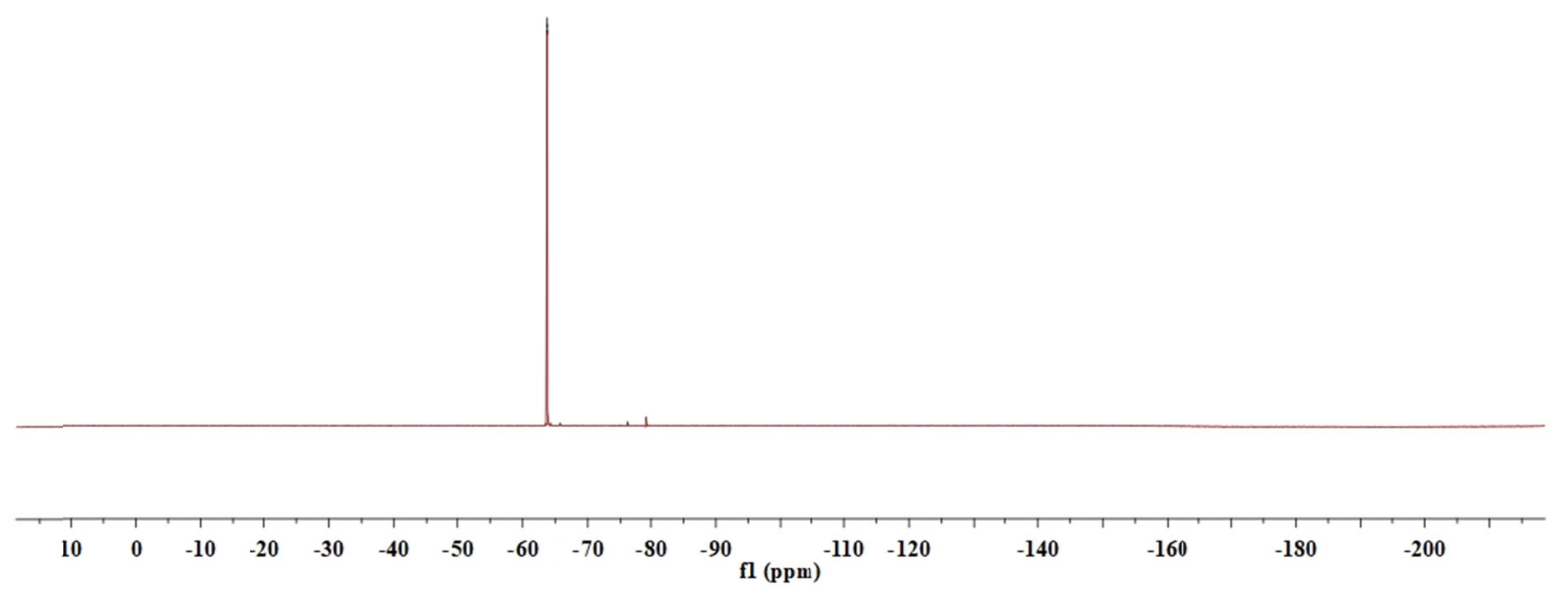



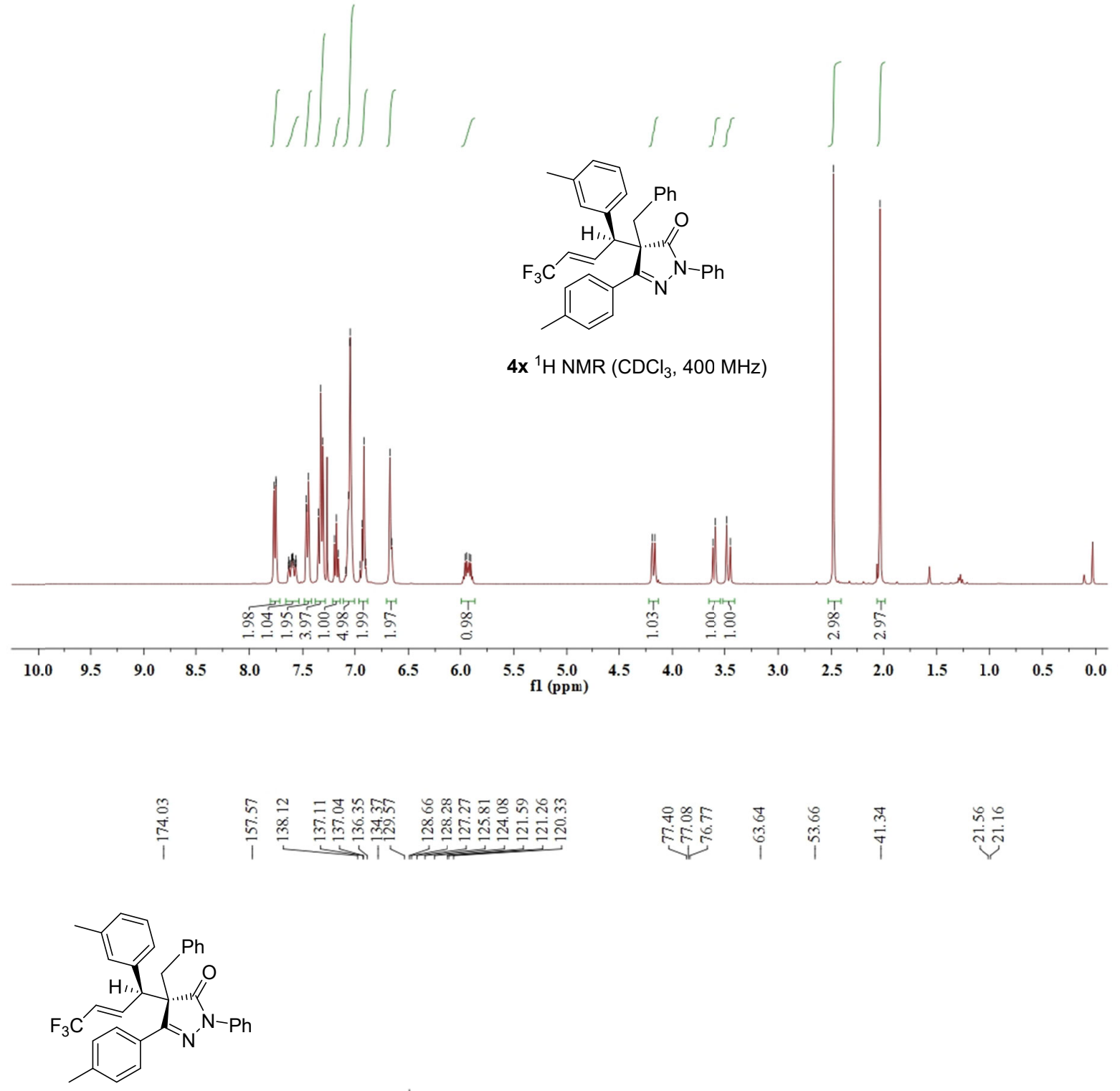

4x ${ }^{13} \mathrm{C} \mathrm{NMR}\left(\mathrm{CDCl}_{3}, 101 \mathrm{MHz}\right)$

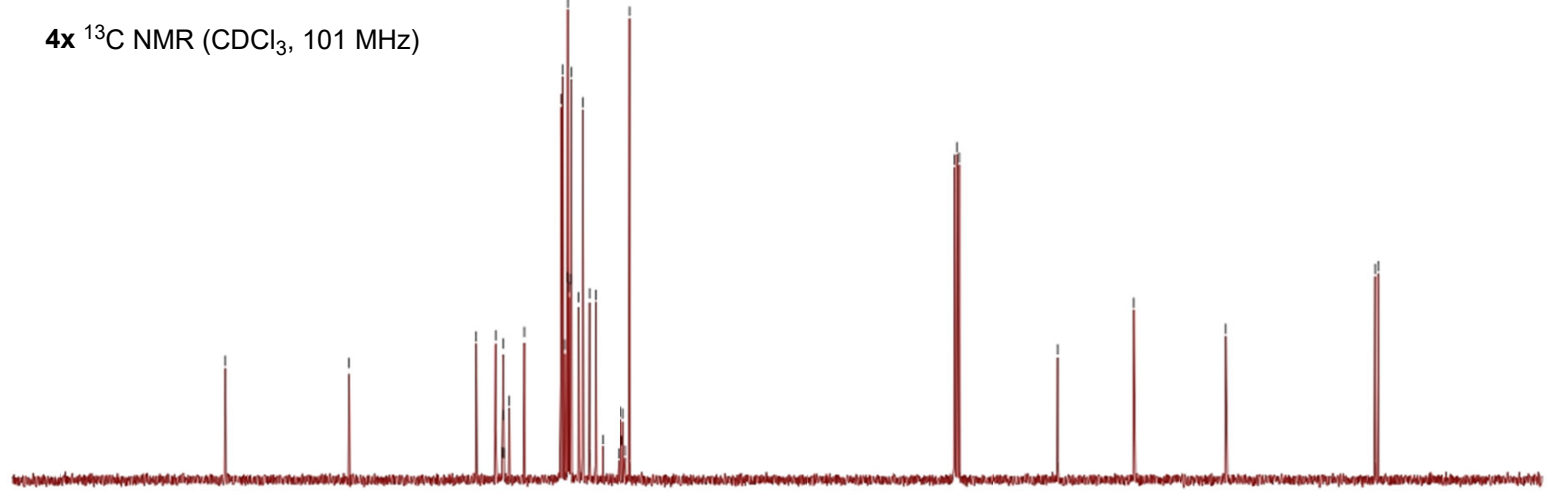




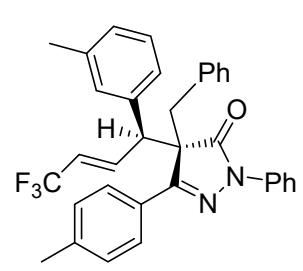

4x ${ }^{19} \mathrm{~F} \mathrm{NMR}\left(\mathrm{CDCl}_{3}, 377 \mathrm{MHz}\right)$

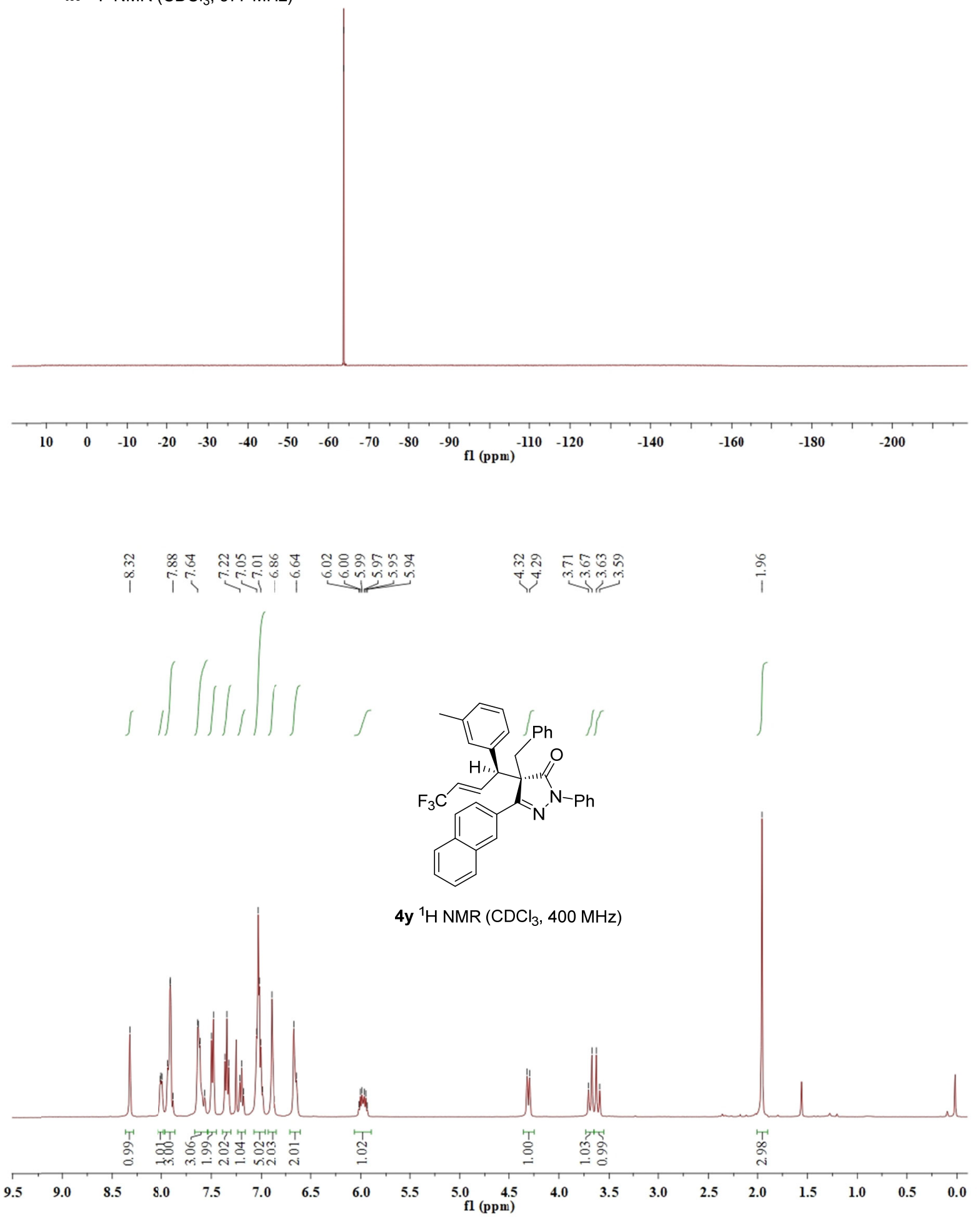



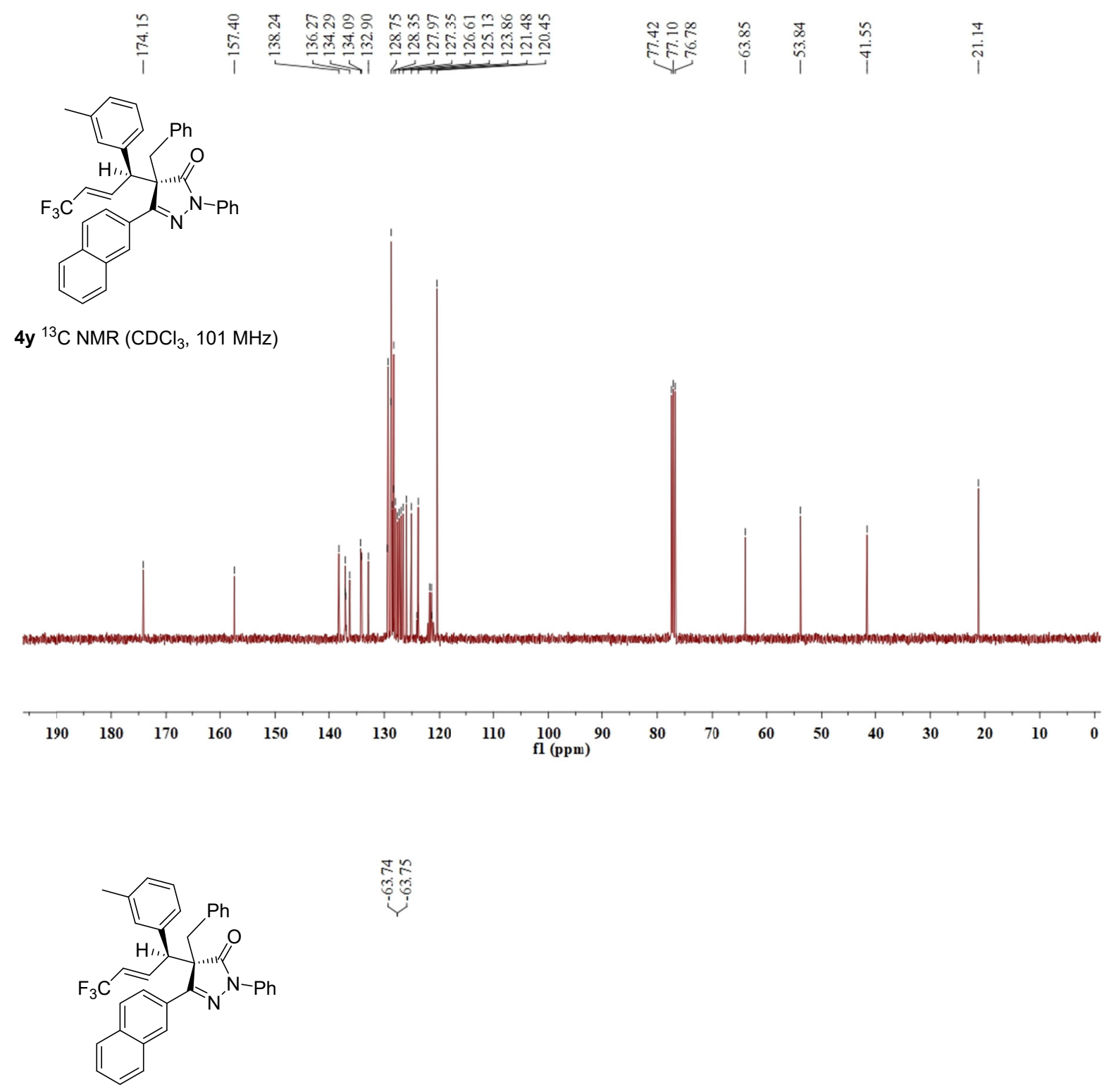

商

4y ${ }^{19} \mathrm{~F} \mathrm{NMR}\left(\mathrm{CDCl}_{3}, 377 \mathrm{MHz}\right)$

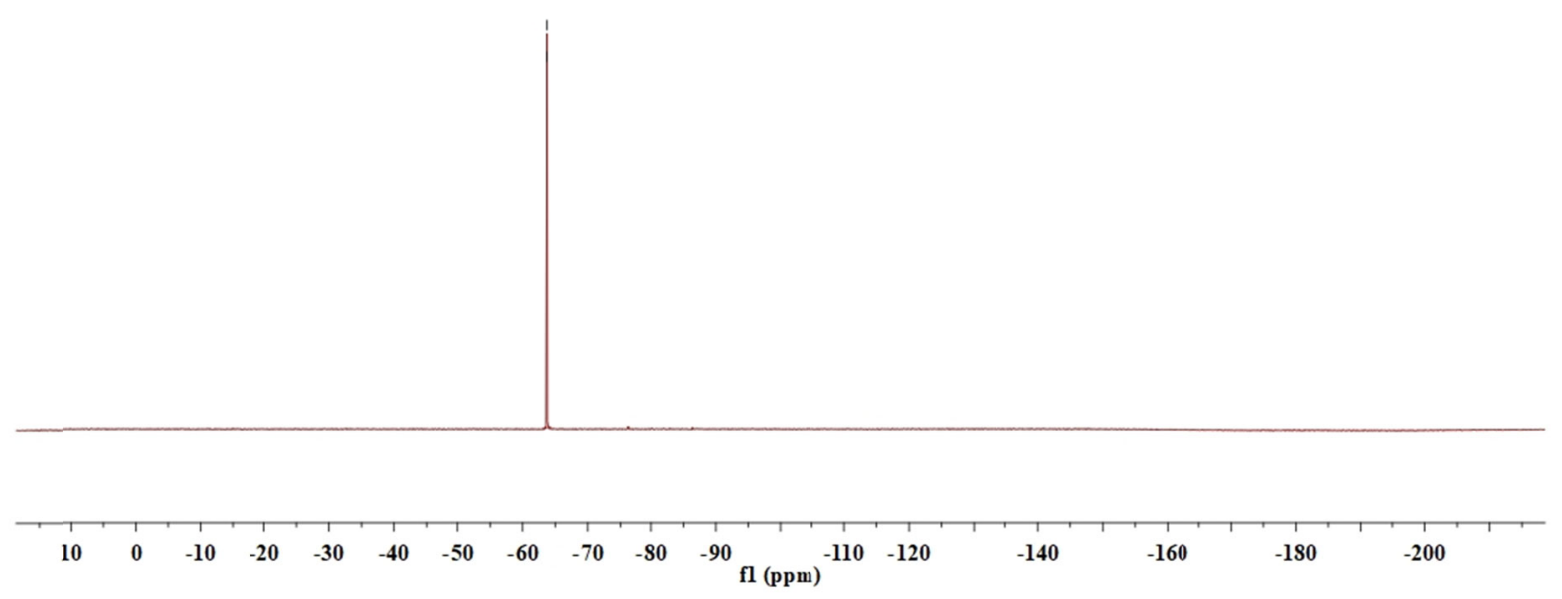



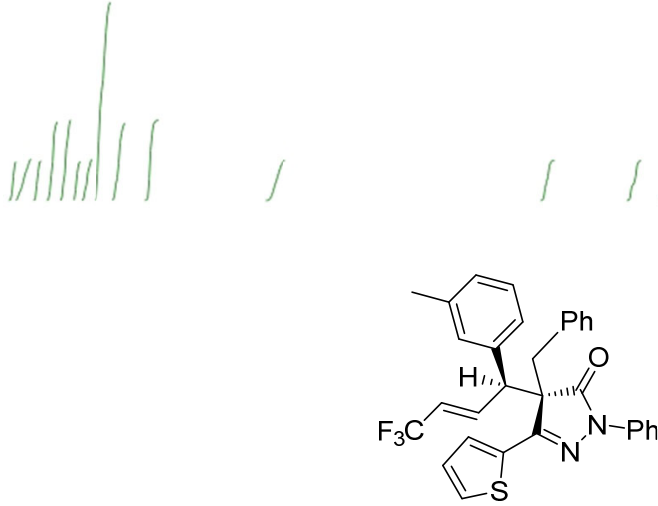

$4 \mathrm{z}^{1} \mathrm{H} \mathrm{NMR}\left(\mathrm{CDCl}_{3}, 400 \mathrm{MHz}\right)$

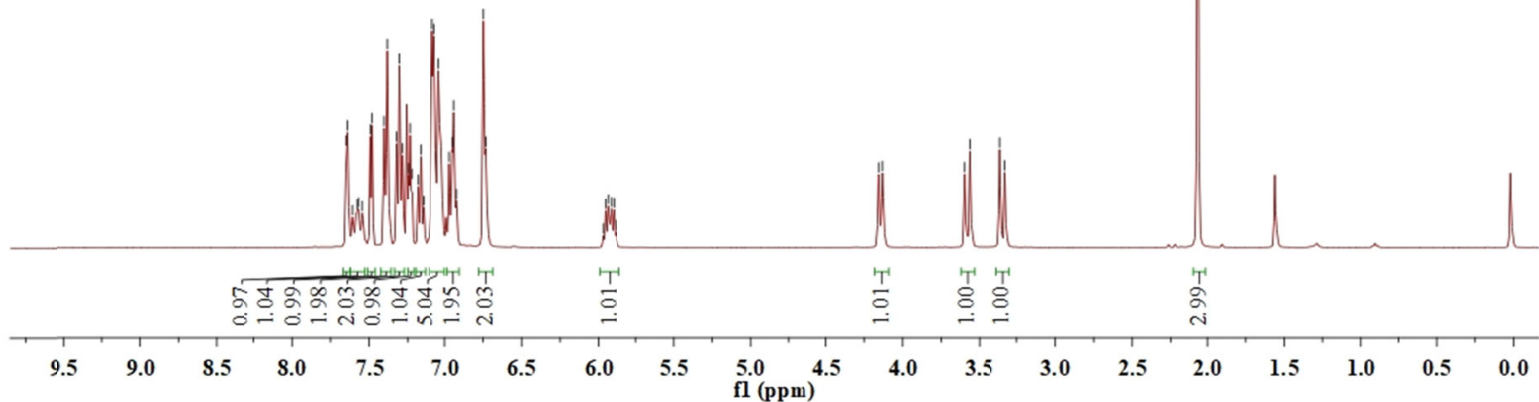

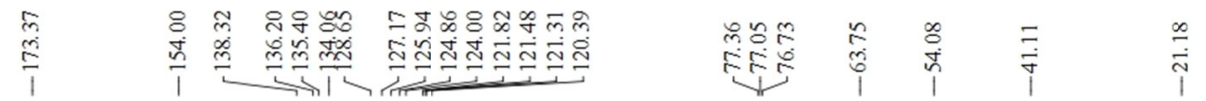

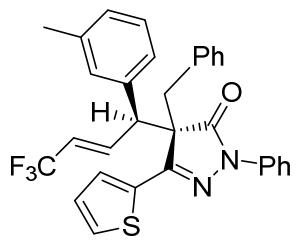

$4 z^{13} \mathrm{C} \mathrm{NMR}\left(\mathrm{CDCl}_{3}, 101 \mathrm{MHz}\right)$

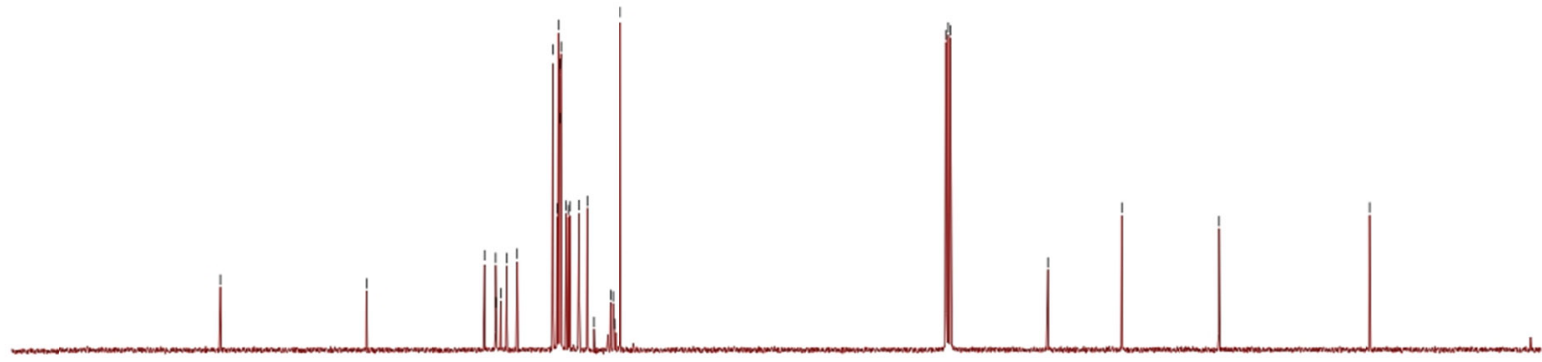




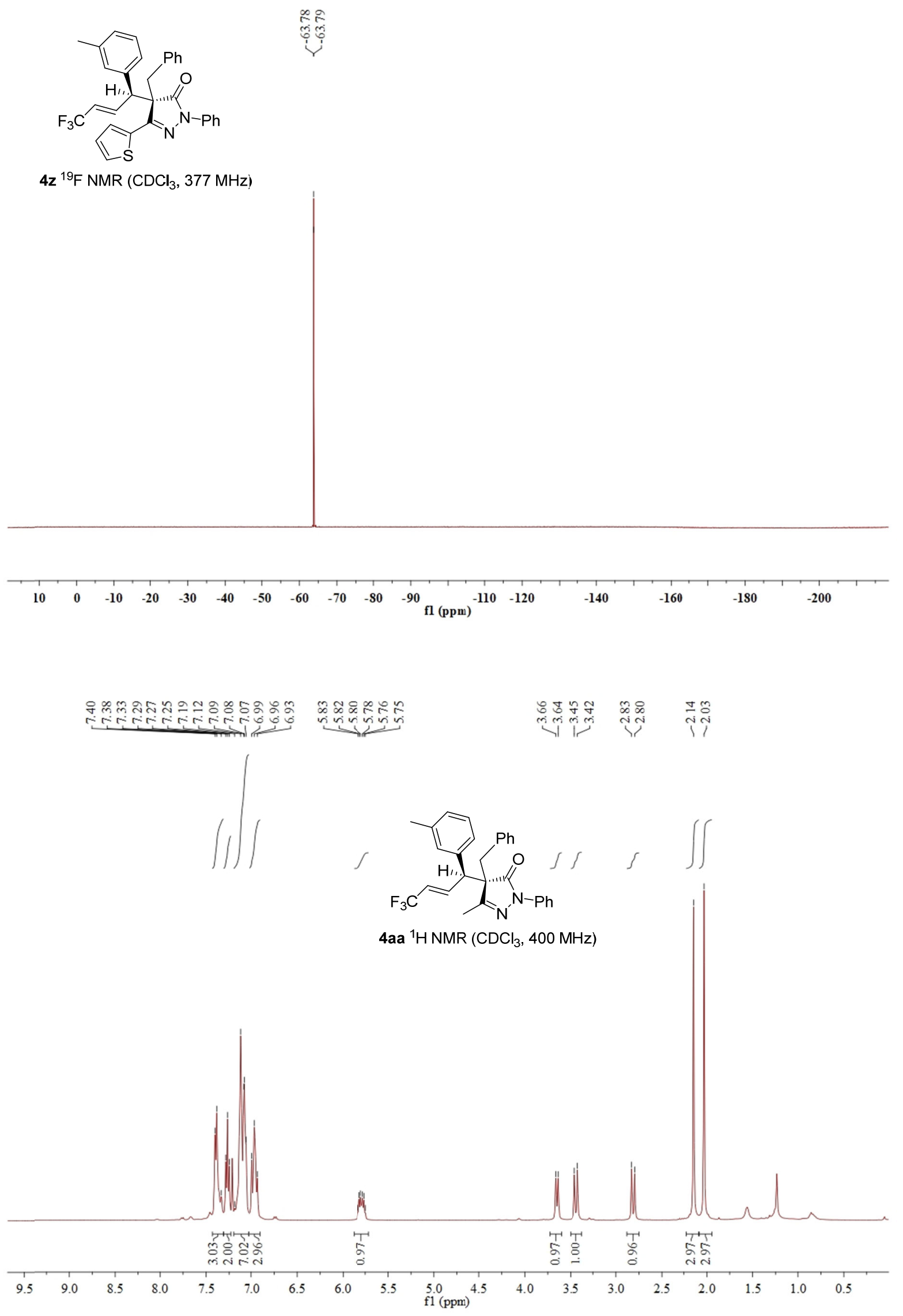




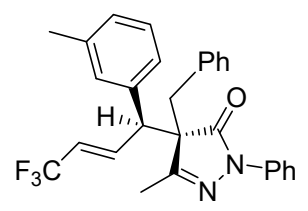

4aa ${ }^{13} \mathrm{C}$ NMR $\left(\mathrm{CDCl}_{3}, 101 \mathrm{MHz}\right)$
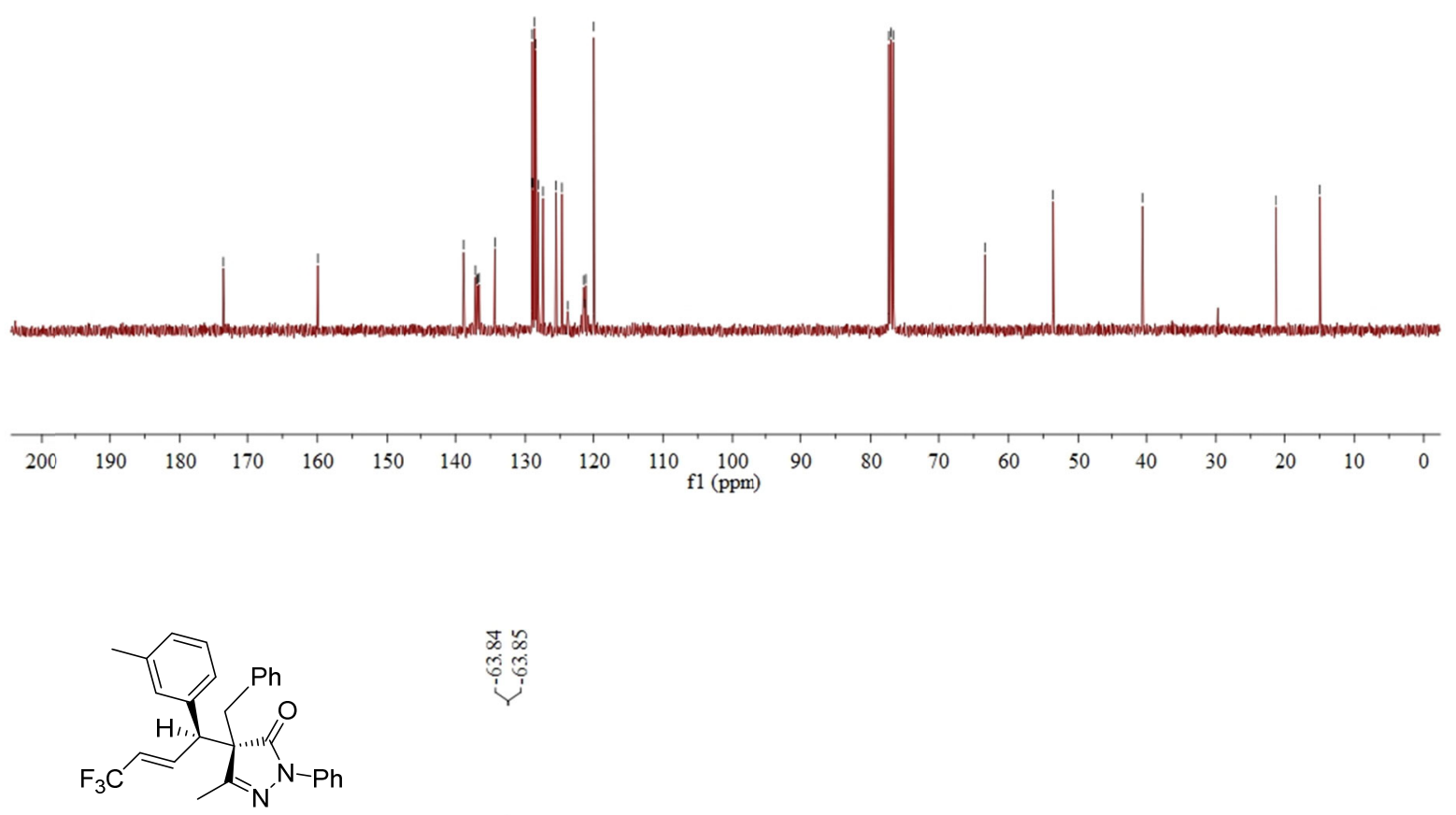

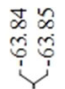

4aa ${ }^{19} \mathrm{~F} \mathrm{NMR}\left(\mathrm{CDCl}_{3}, 377 \mathrm{MHz}\right)$

$\begin{array}{llllllllllllllllllllll}1 & 0 & 0 & -10 & -20 & -30 & -40 & -50 & -60 & -70 & -80 & -90 & & -110 & -120 & -140 & -160 & -180 & -200\end{array}$




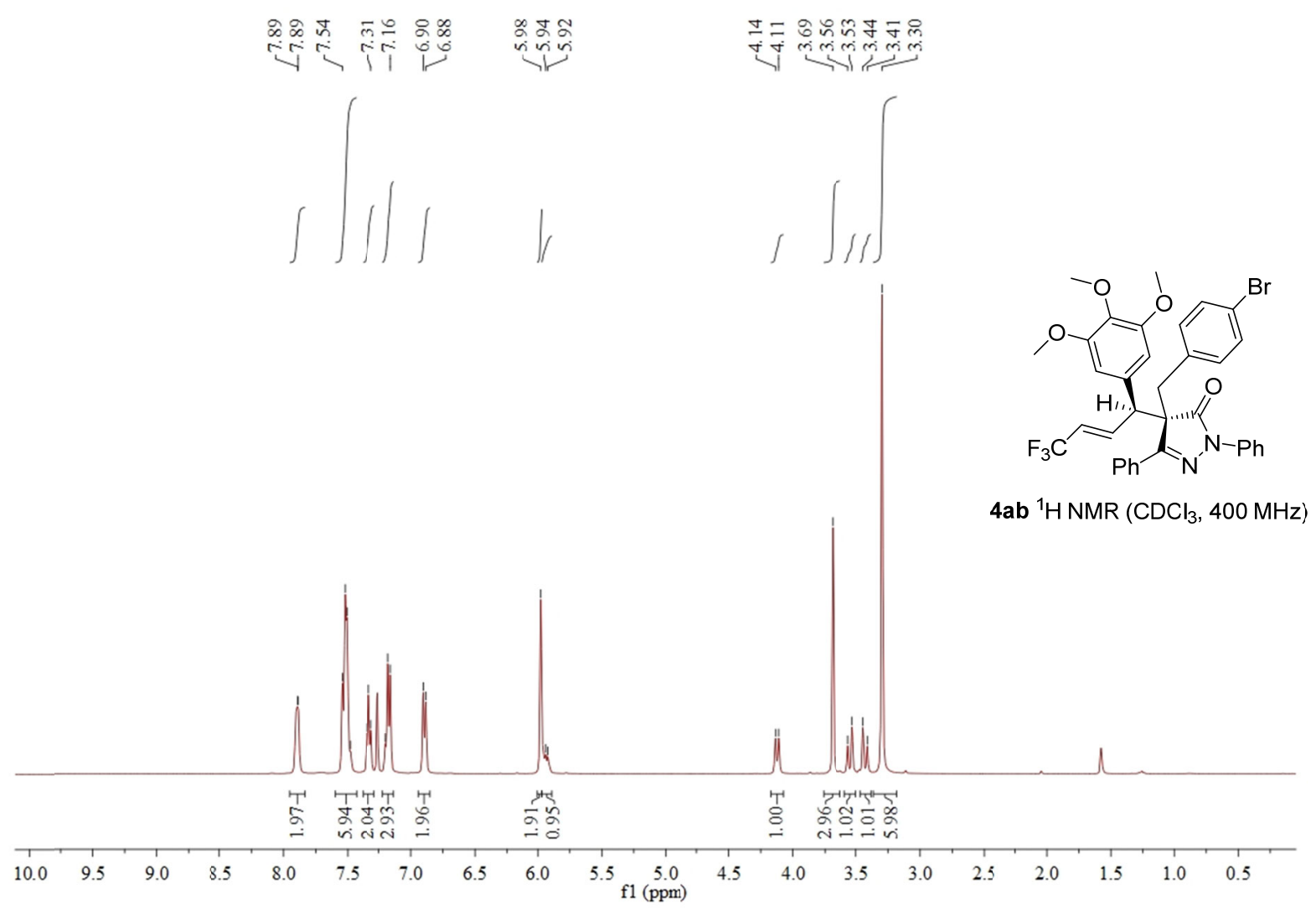

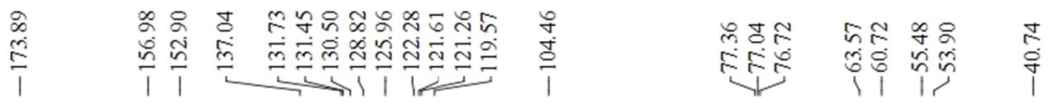

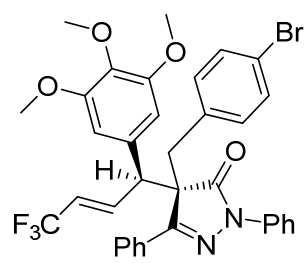

4ab ${ }^{13} \mathrm{C}$ NMR $\left(\mathrm{CDCl}_{3}, 101 \mathrm{MHz}\right)$

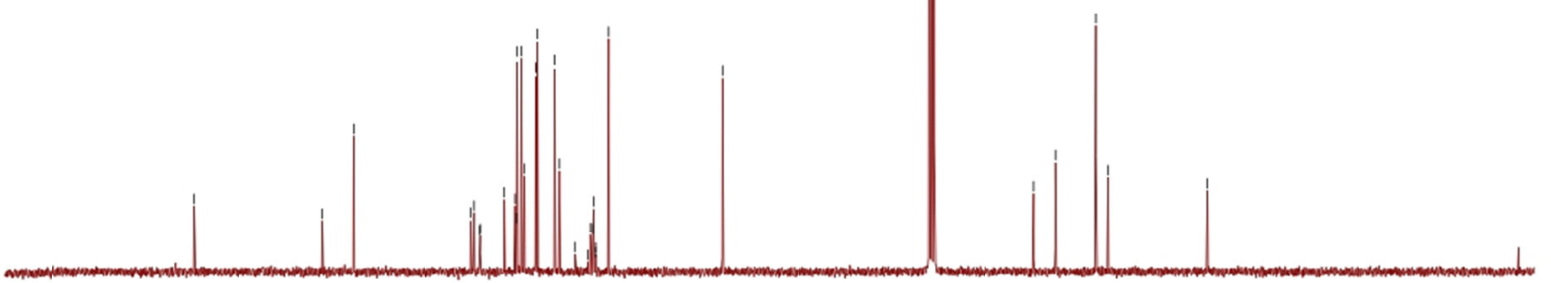

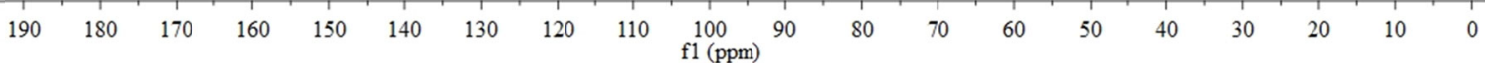




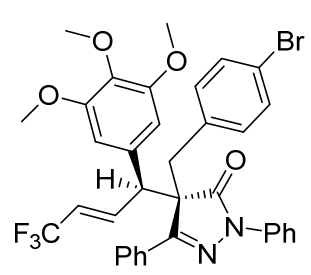

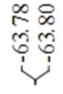

4ab ${ }^{19} \mathrm{~F}$ NMR $\left(\mathrm{CDCl}_{3}, 377 \mathrm{MHz}\right)$
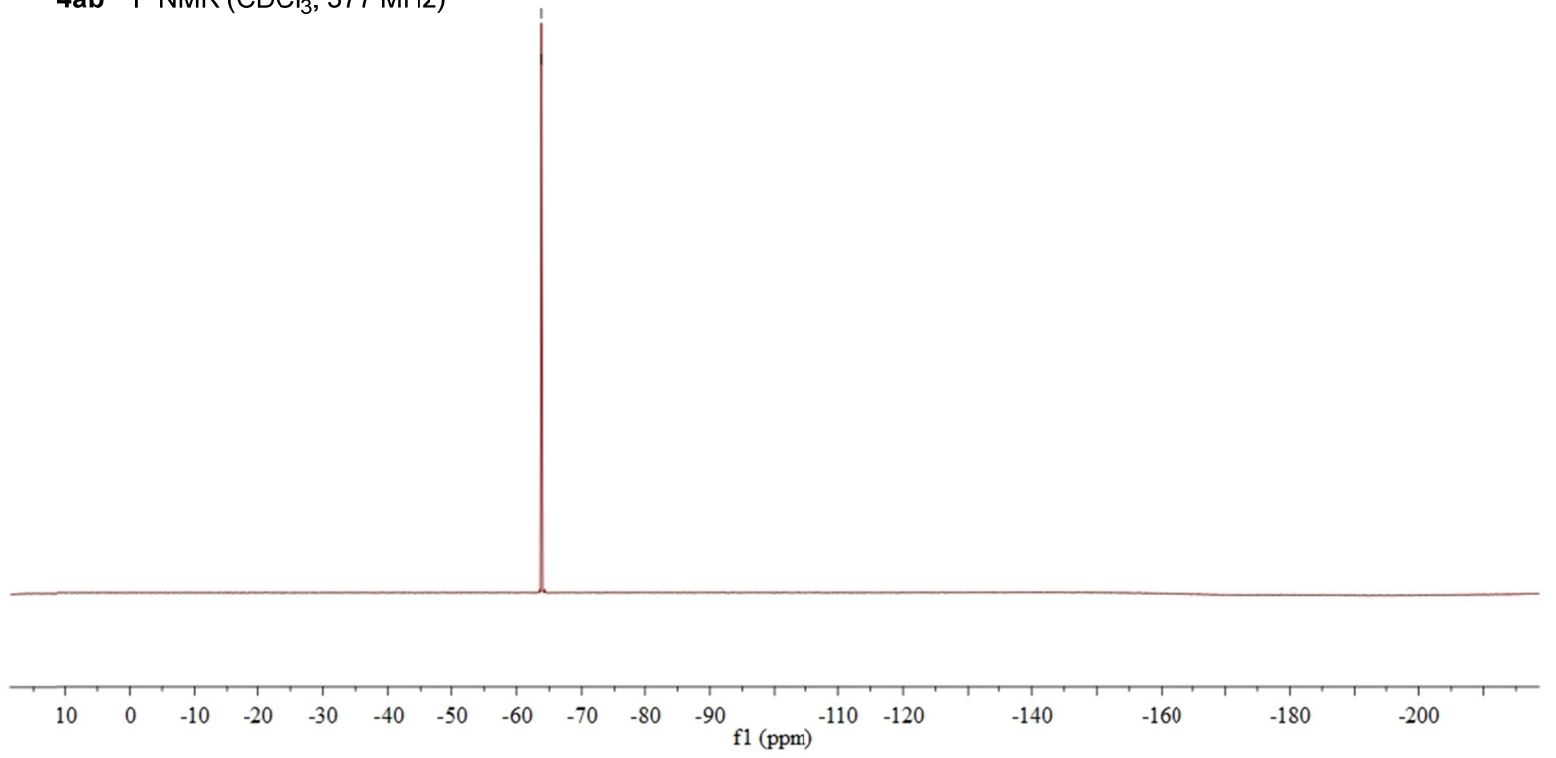

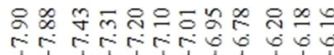

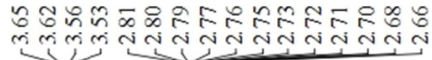
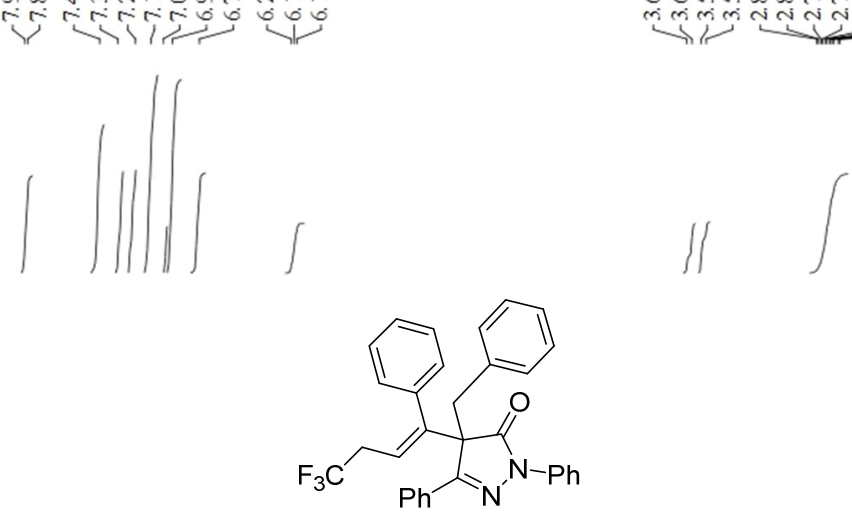

5a ${ }^{1} \mathrm{H}$ NMR $\left(\mathrm{CDCl}_{3}, 400 \mathrm{MHz}\right)$

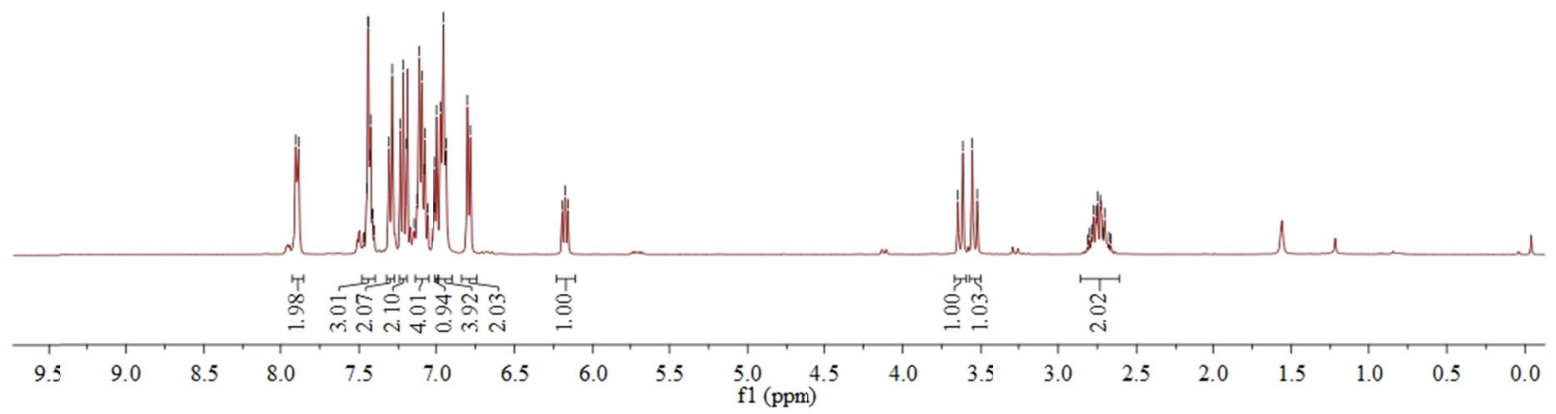




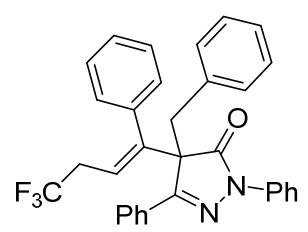

$5 \mathbf{a}^{13} \mathrm{C} \mathrm{NMR}\left(\mathrm{CDCl}_{3}, 101 \mathrm{MHz}\right)$

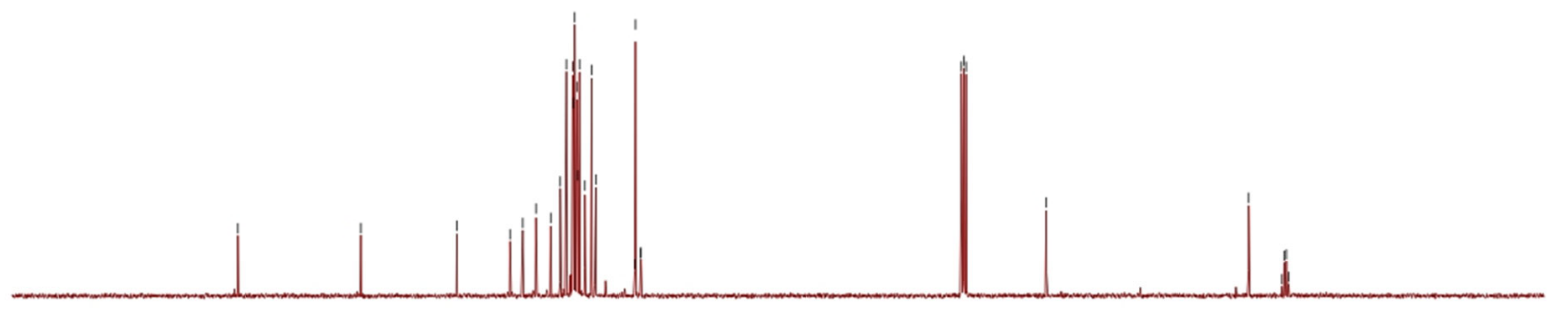

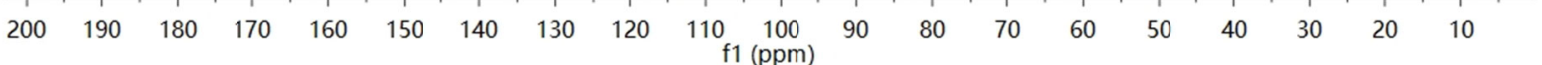

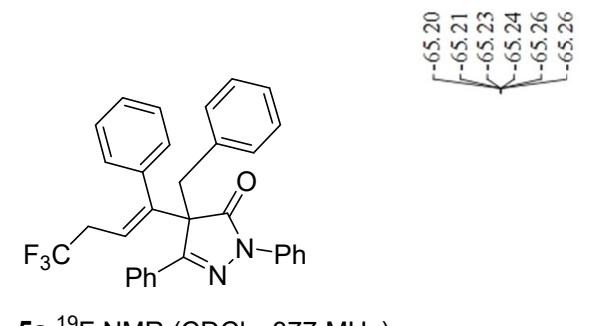

5a ${ }^{19} \mathrm{~F}$ NMR $\left(\mathrm{CDCl}_{3}, 377 \mathrm{MHz}\right)$

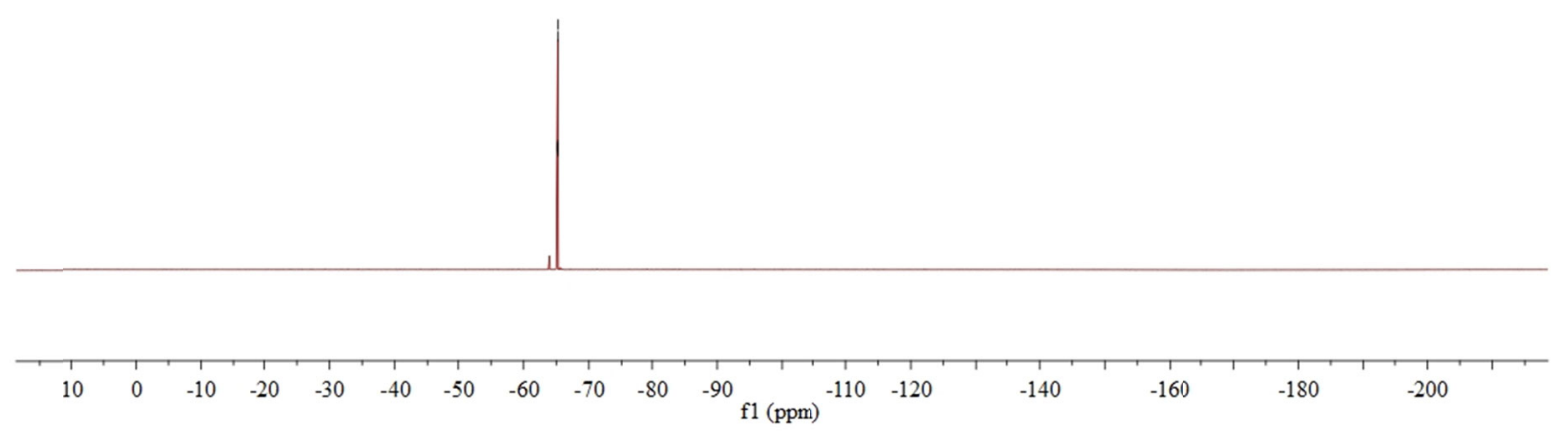




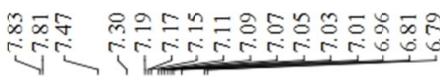

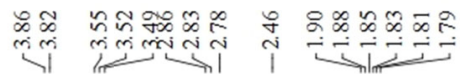
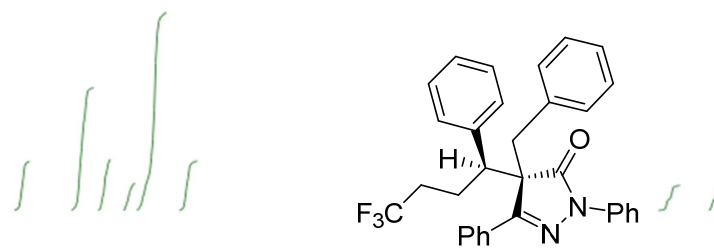

6a ${ }^{1} \mathrm{H} \mathrm{NMR}\left(\mathrm{CDCl}_{3}, 400 \mathrm{MHz}\right)$

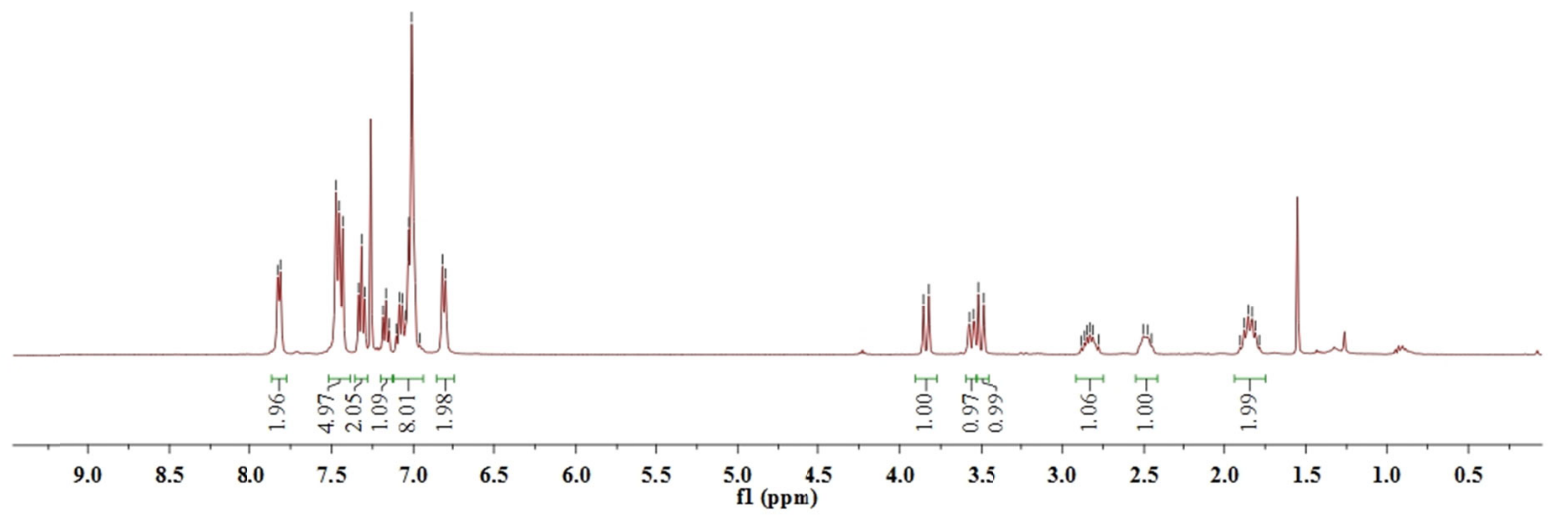

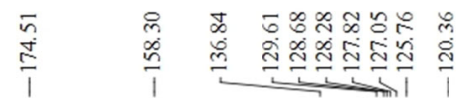

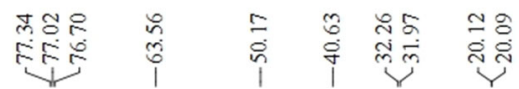

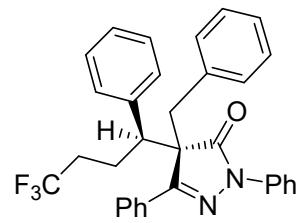

6a ${ }^{13} \mathrm{C} \mathrm{NMR}\left(\mathrm{CDCl}_{3}, 101 \mathrm{MHz}\right)$

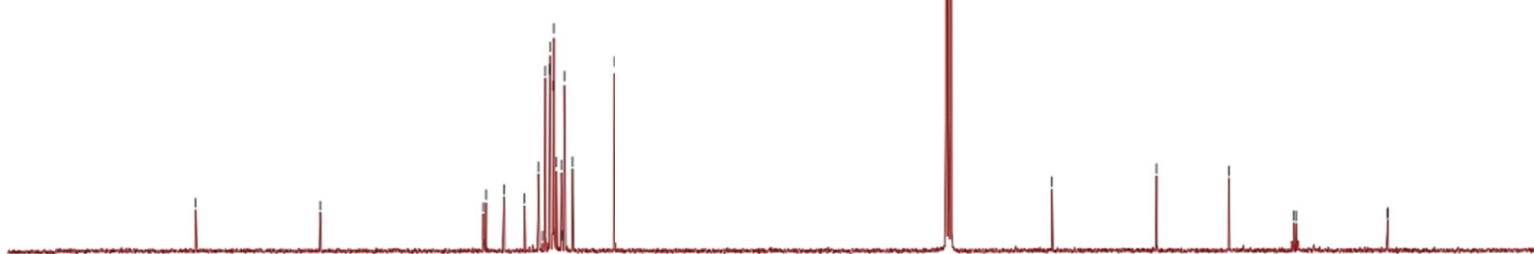

$\begin{array}{lllllll}170 & 160 & 150 & 140 & 130 & 120\end{array}$

100
$\mathrm{f} 1(\mathrm{ppm})$ 


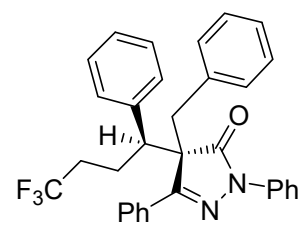

6a ${ }^{19} \mathrm{~F} \mathrm{NMR}\left(\mathrm{CDCl}_{3}, 377 \mathrm{MHz}\right)$

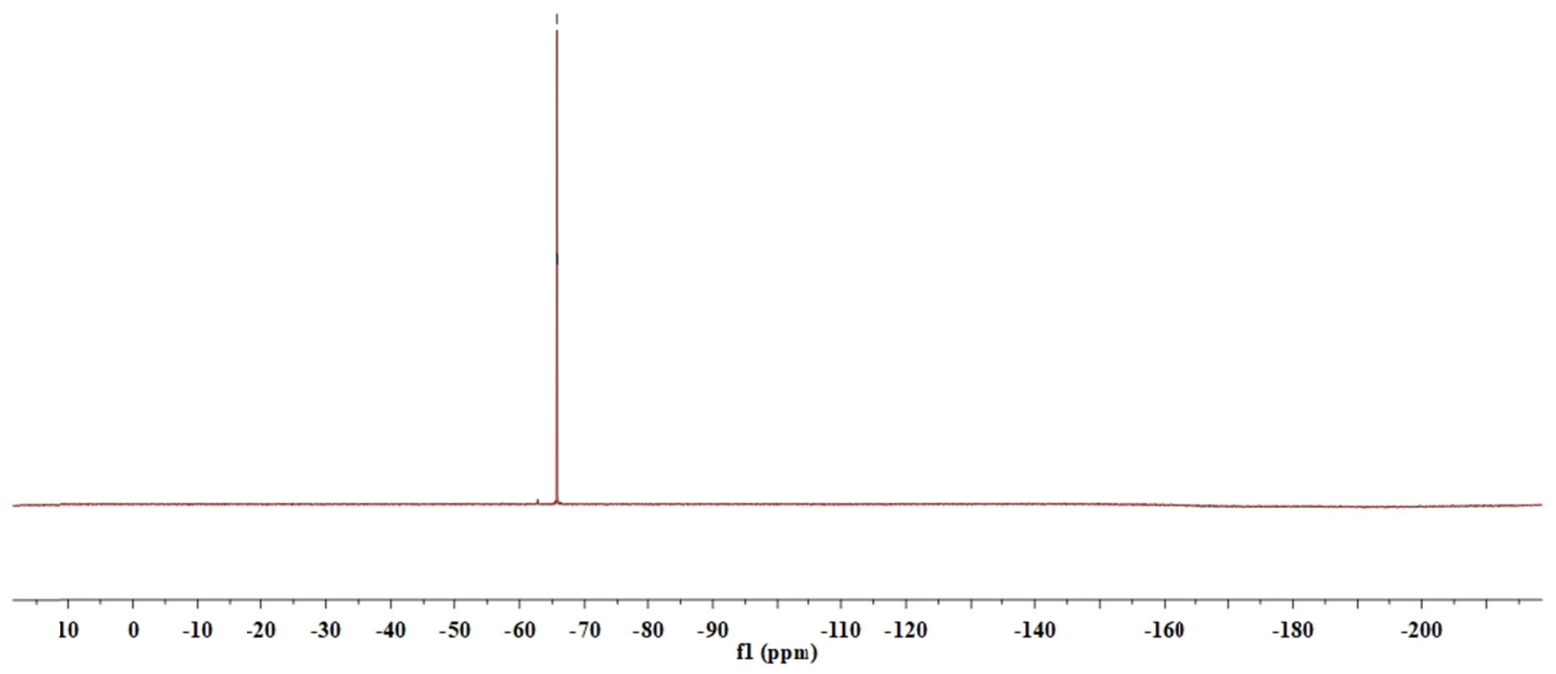

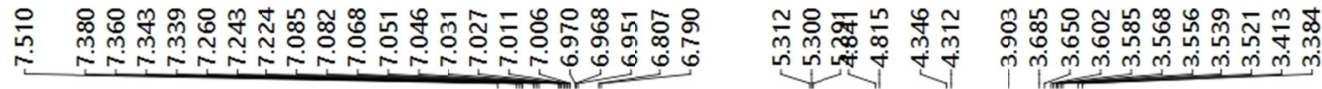

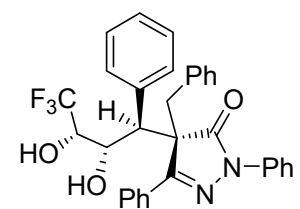

$7 \mathrm{a}{ }^{1} \mathrm{H} \mathrm{NMR}\left(\mathrm{CDCl}_{3}, 400 \mathrm{MHz}\right)$

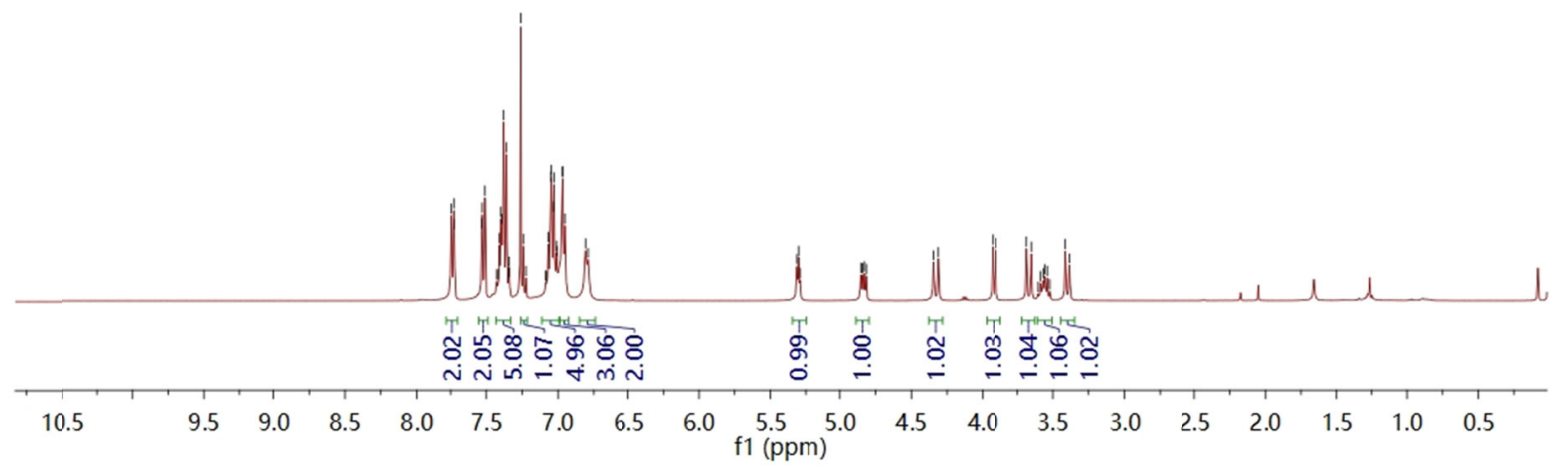




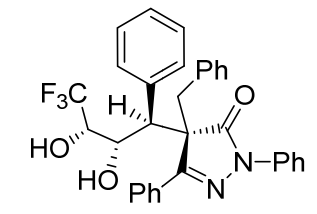

7a Hydrogen-deuterium exchange

${ }^{1} \mathrm{H} \mathrm{NMR}\left(\mathrm{CDCl}_{3}, 400 \mathrm{MHz}\right)$
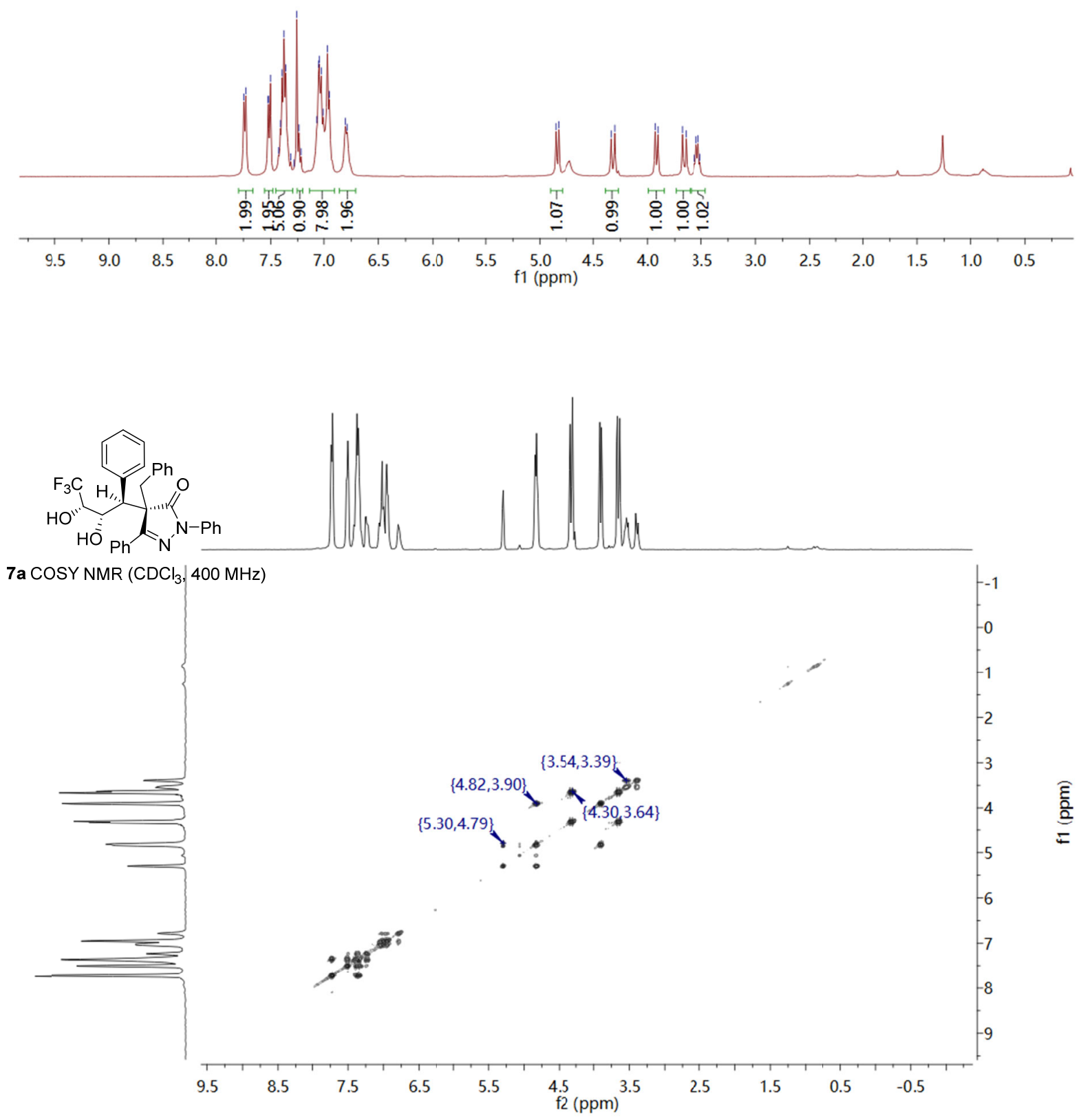


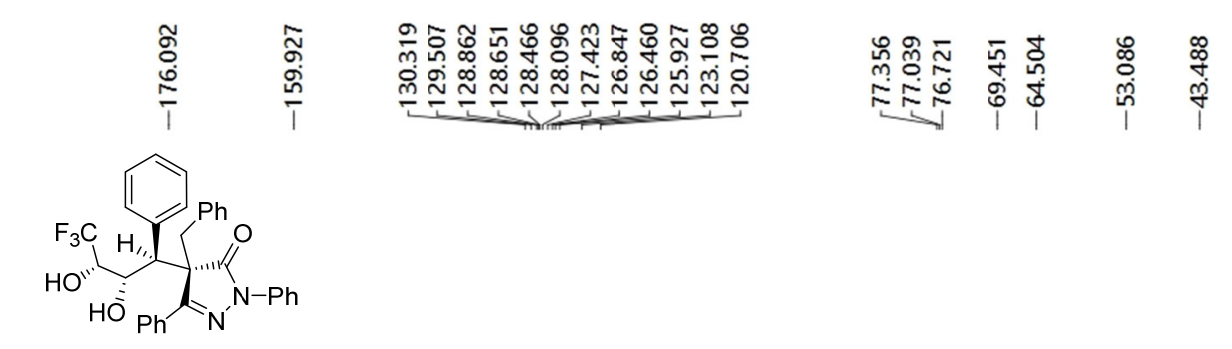

$\mathbf{7 a}^{13} \mathrm{C}$ NMR $\left(\mathrm{CDCl}_{3}, 101 \mathrm{MHz}\right)$
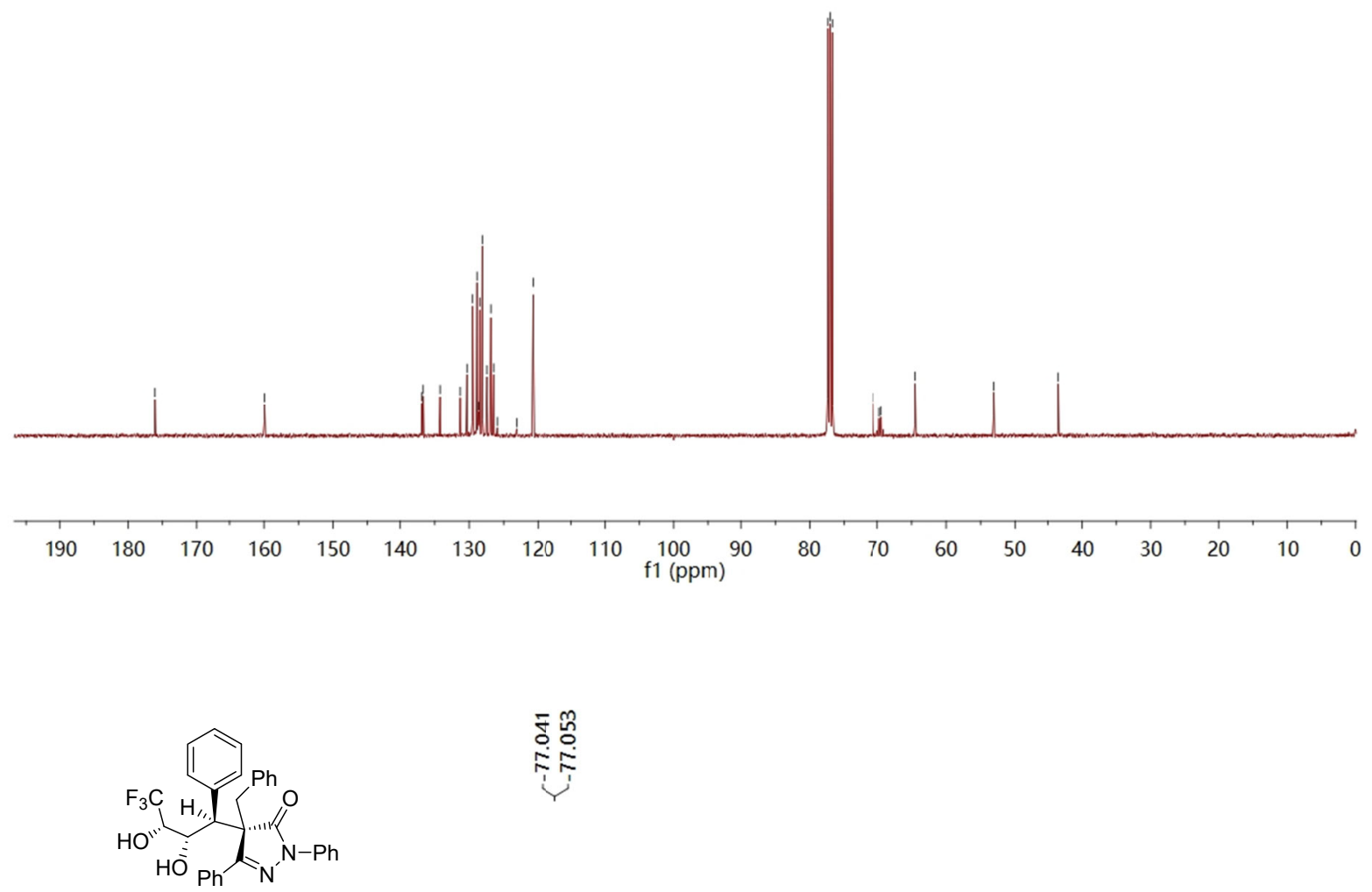

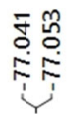

7a ${ }^{19} \mathrm{~F} \mathrm{NMR}\left(\mathrm{CDCl}_{3}, 377 \mathrm{MHz}\right)$

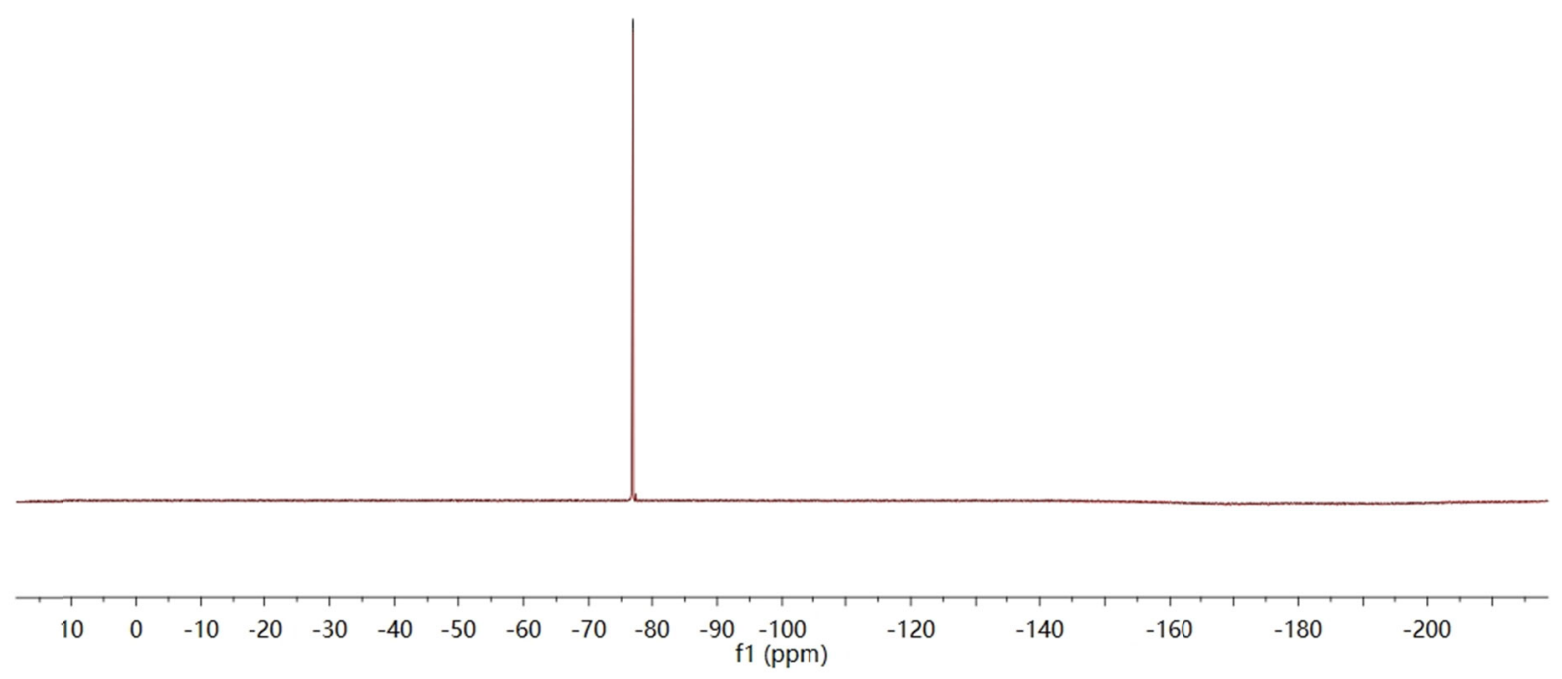




\section{Copies of HPLC spectrum}
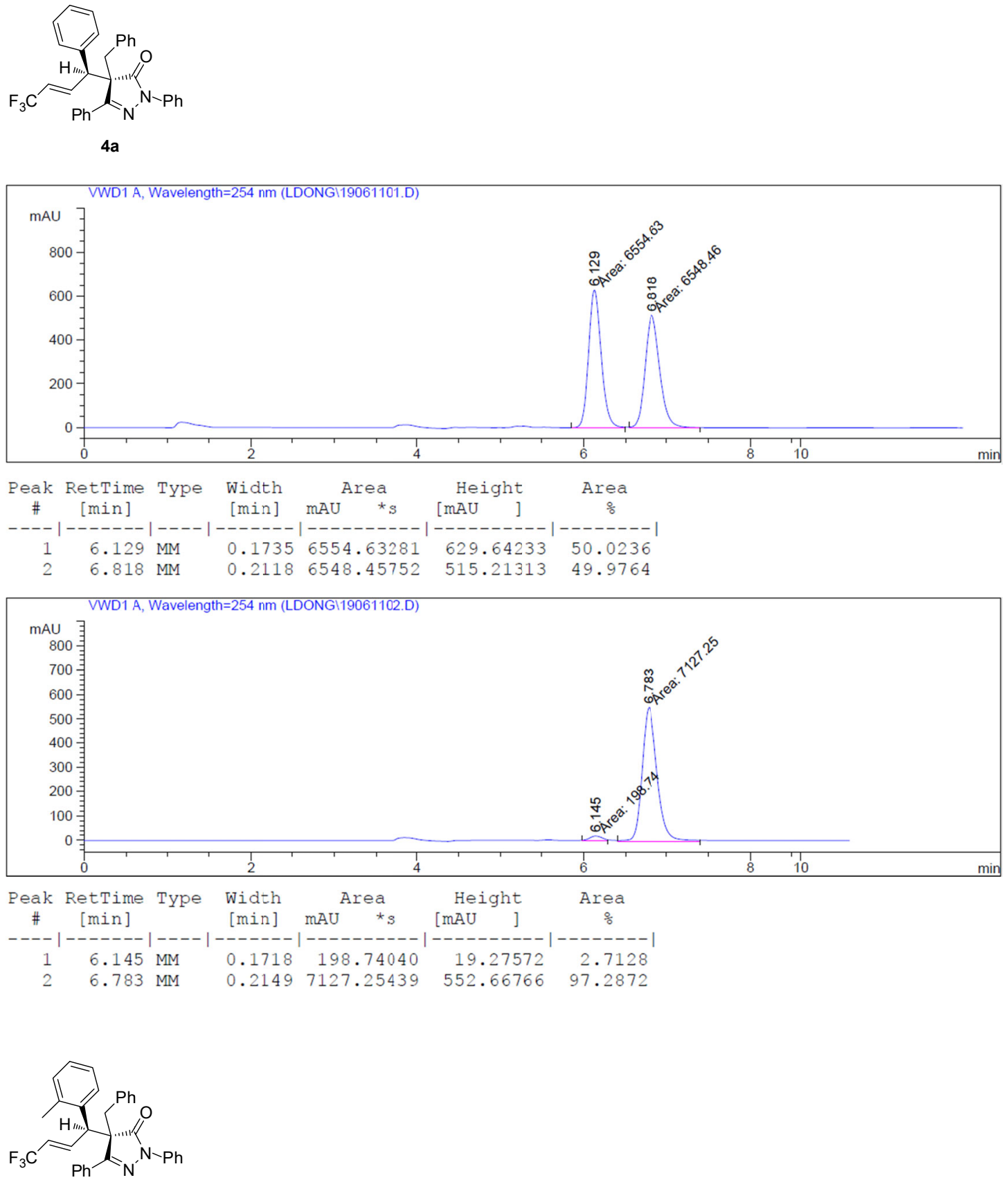

4b

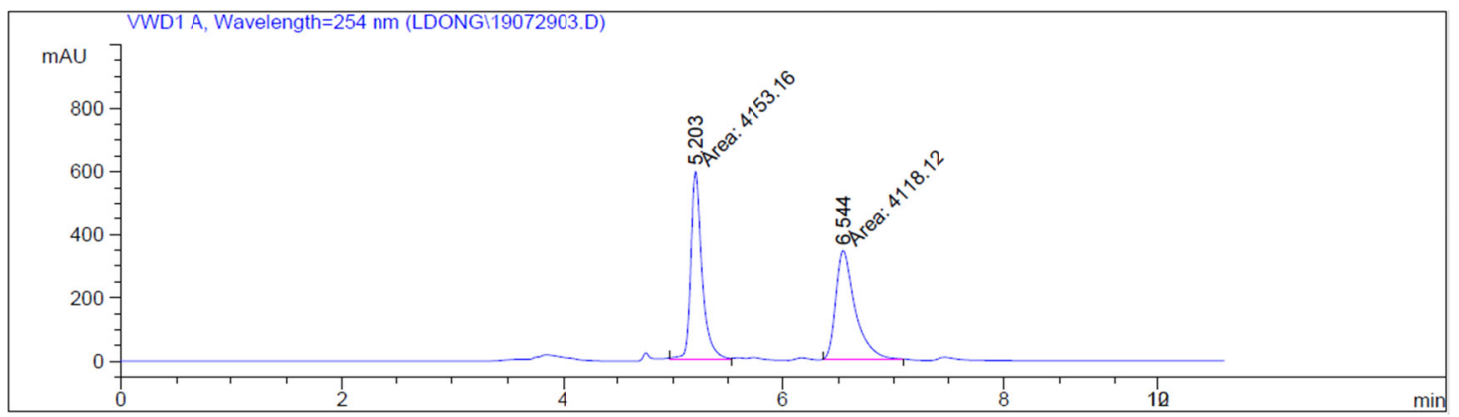



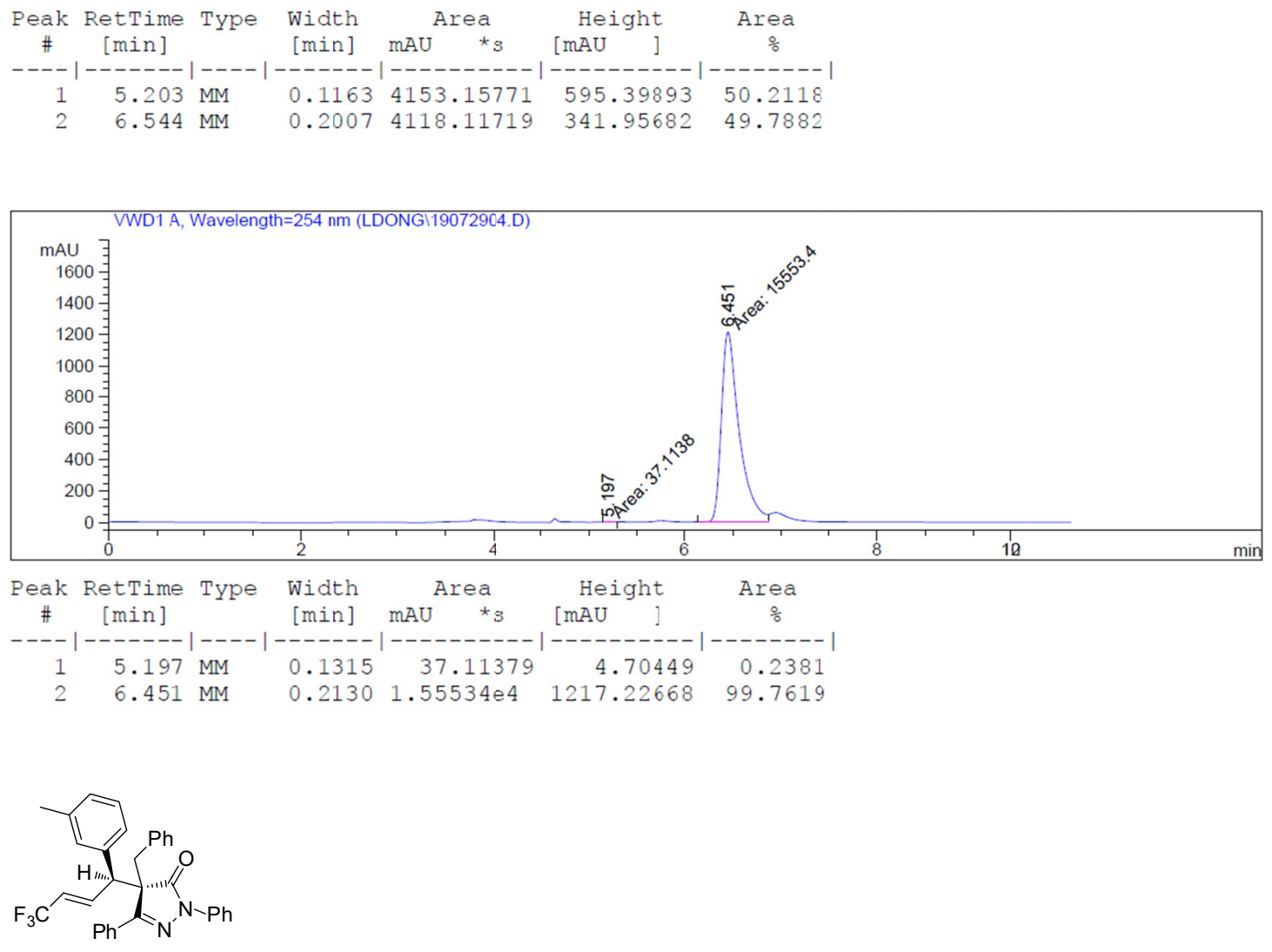

4c
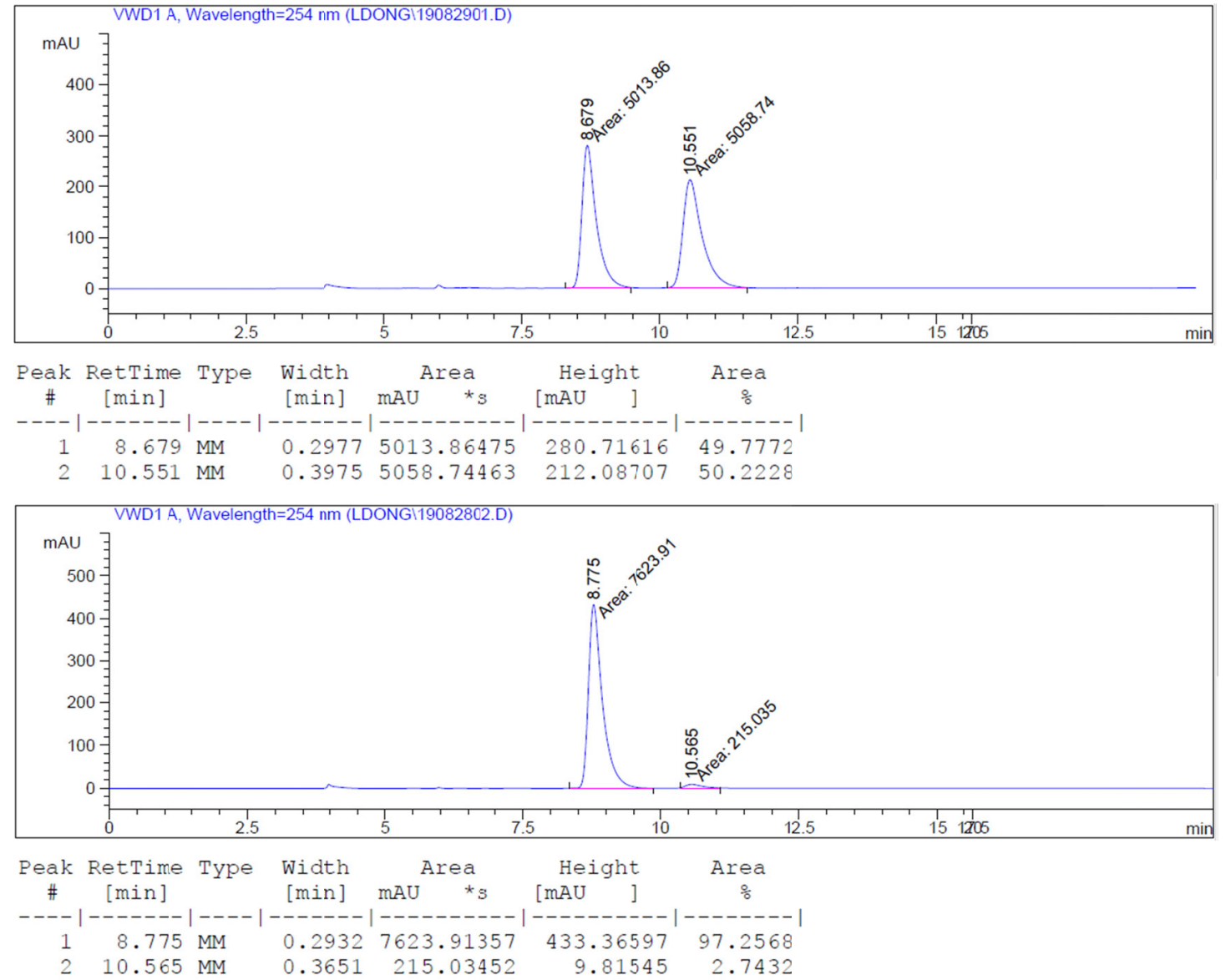

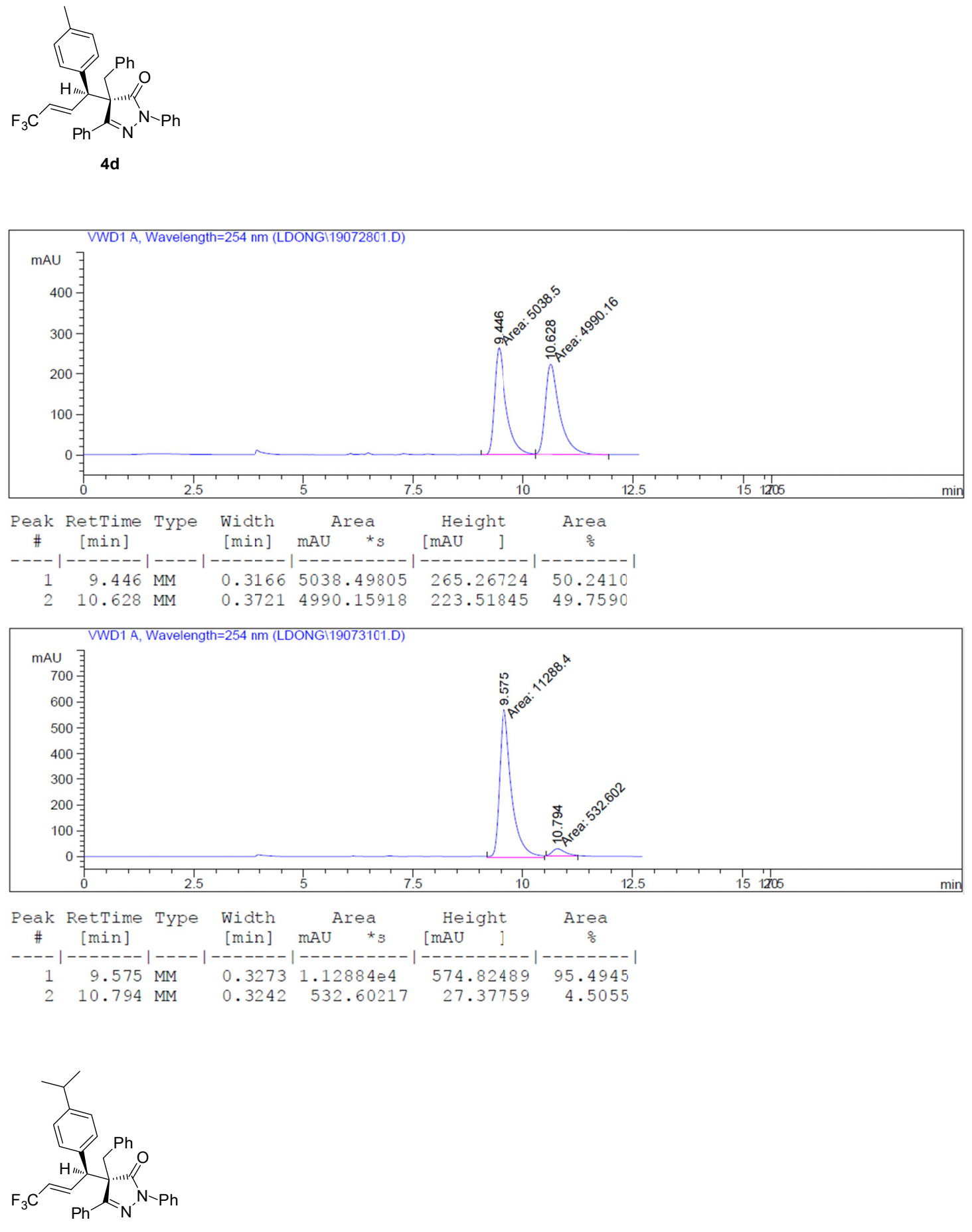

$4 e$

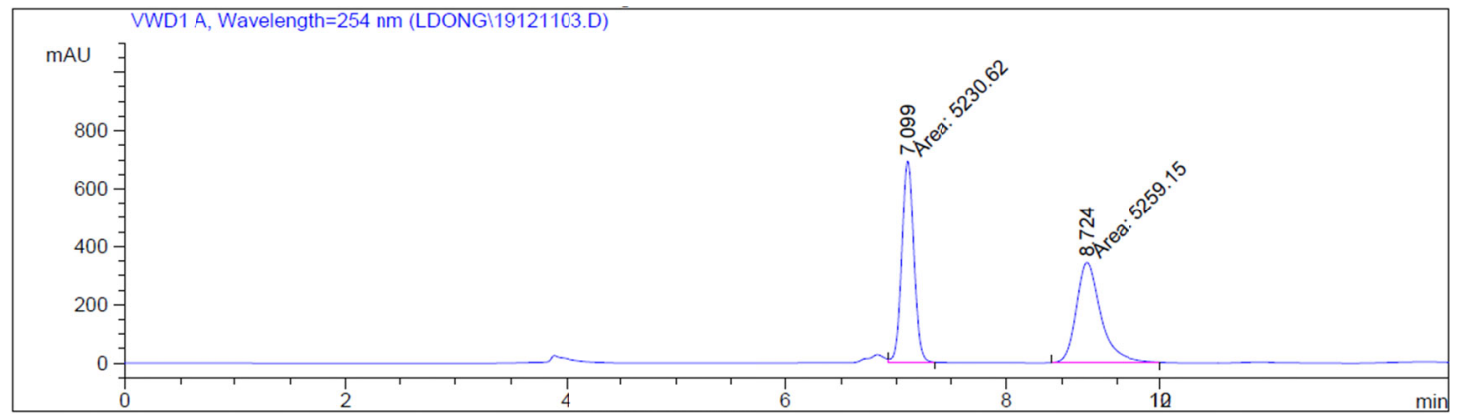



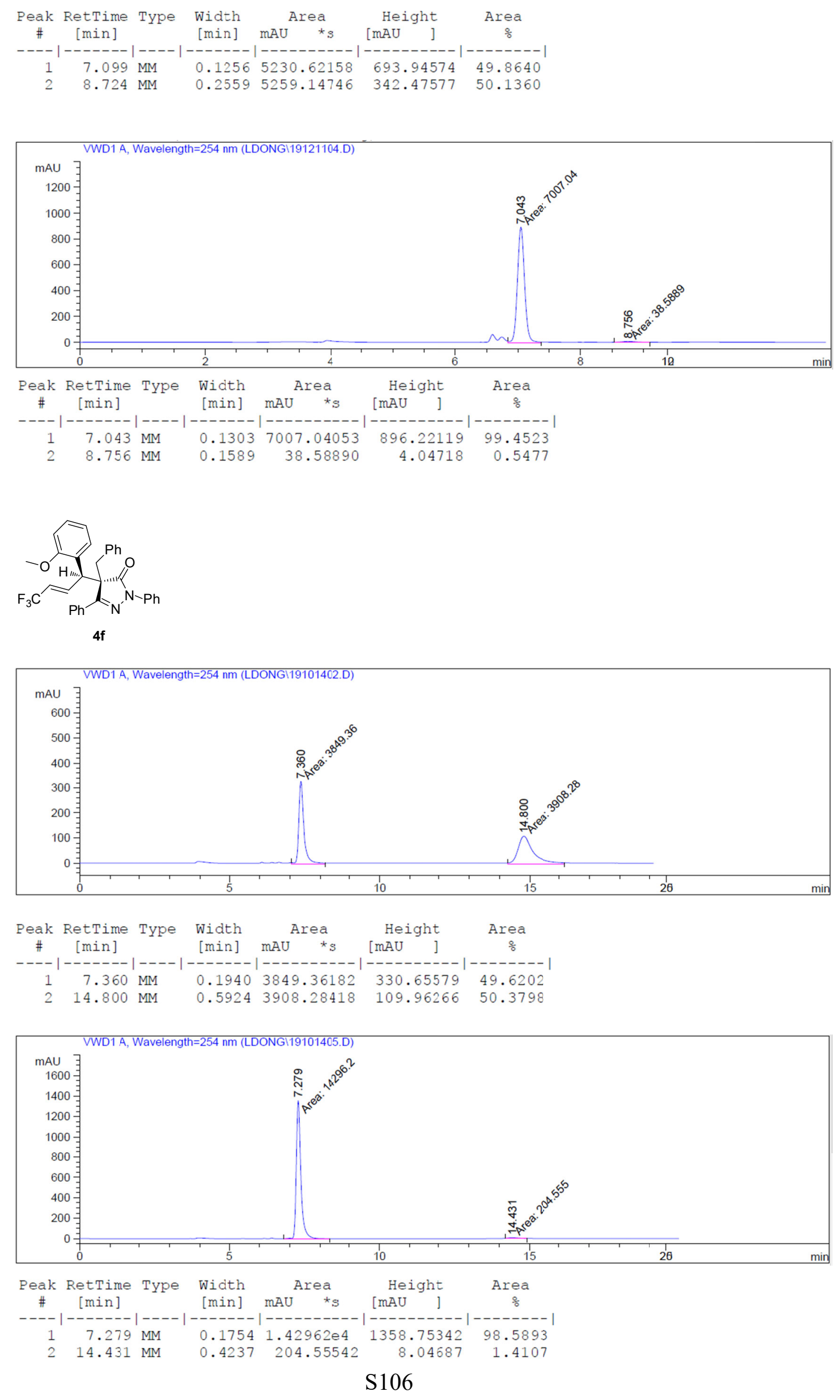


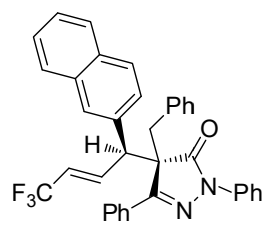

$4 \mathrm{~g}$
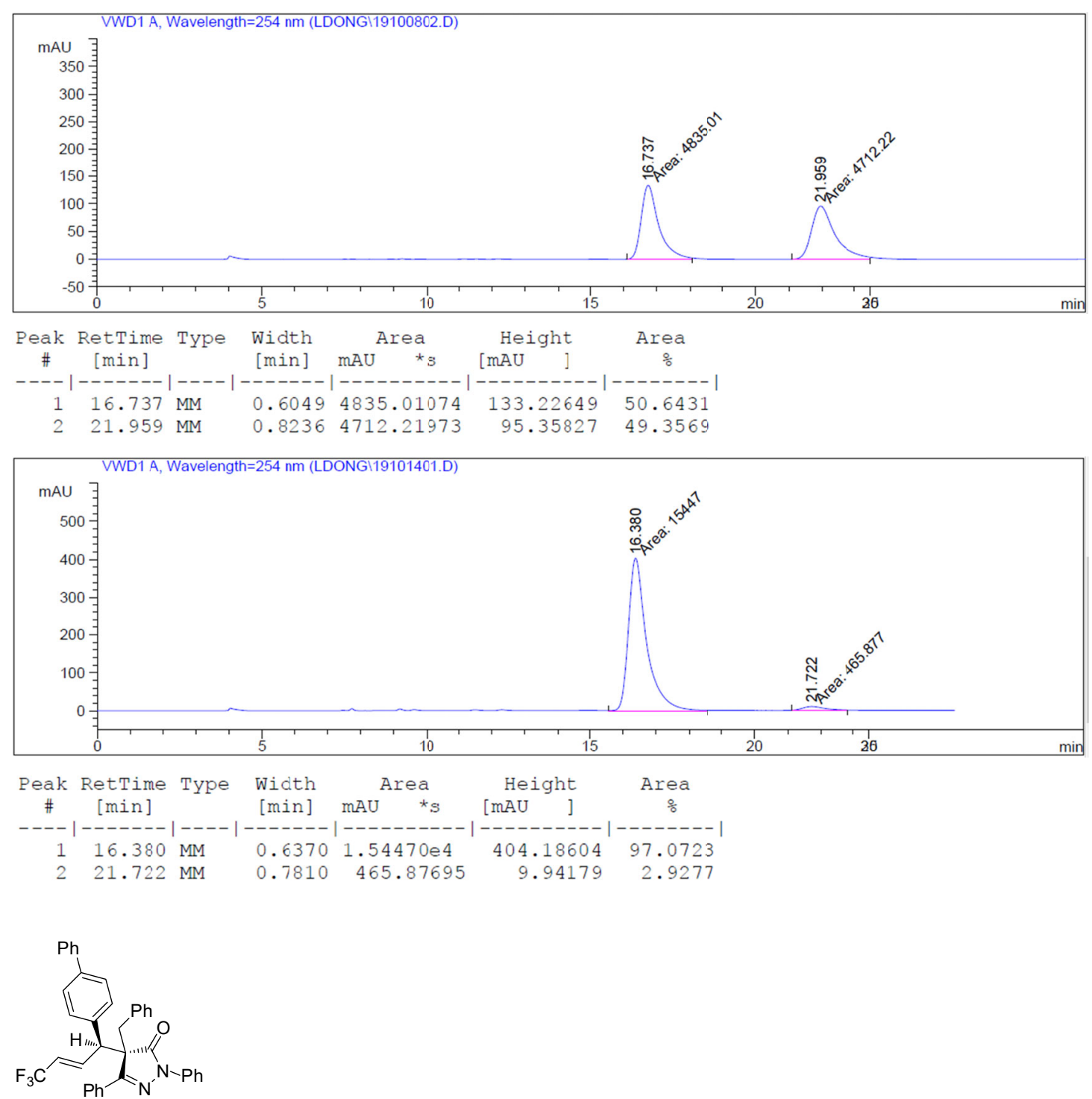

4h

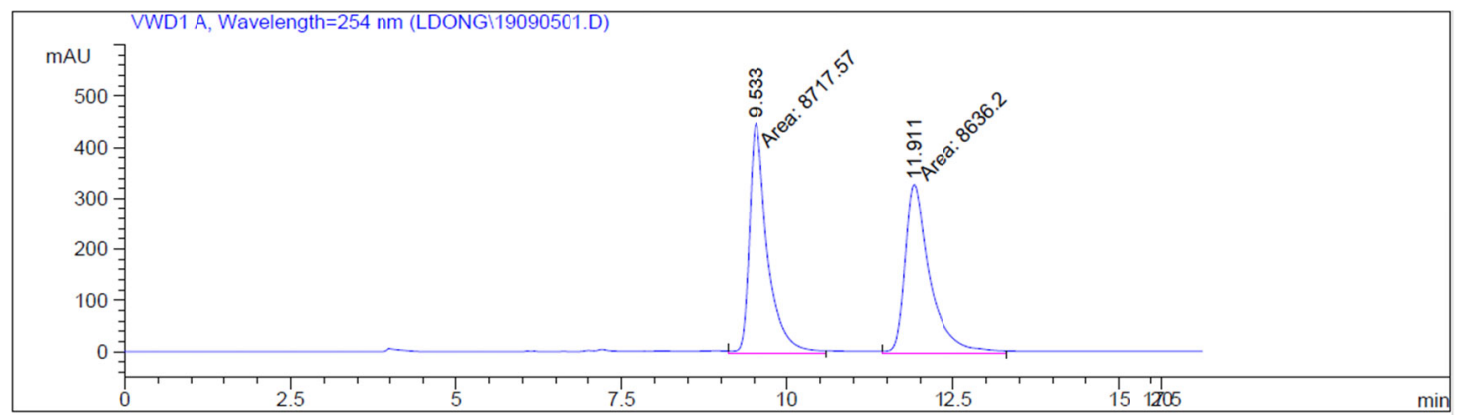



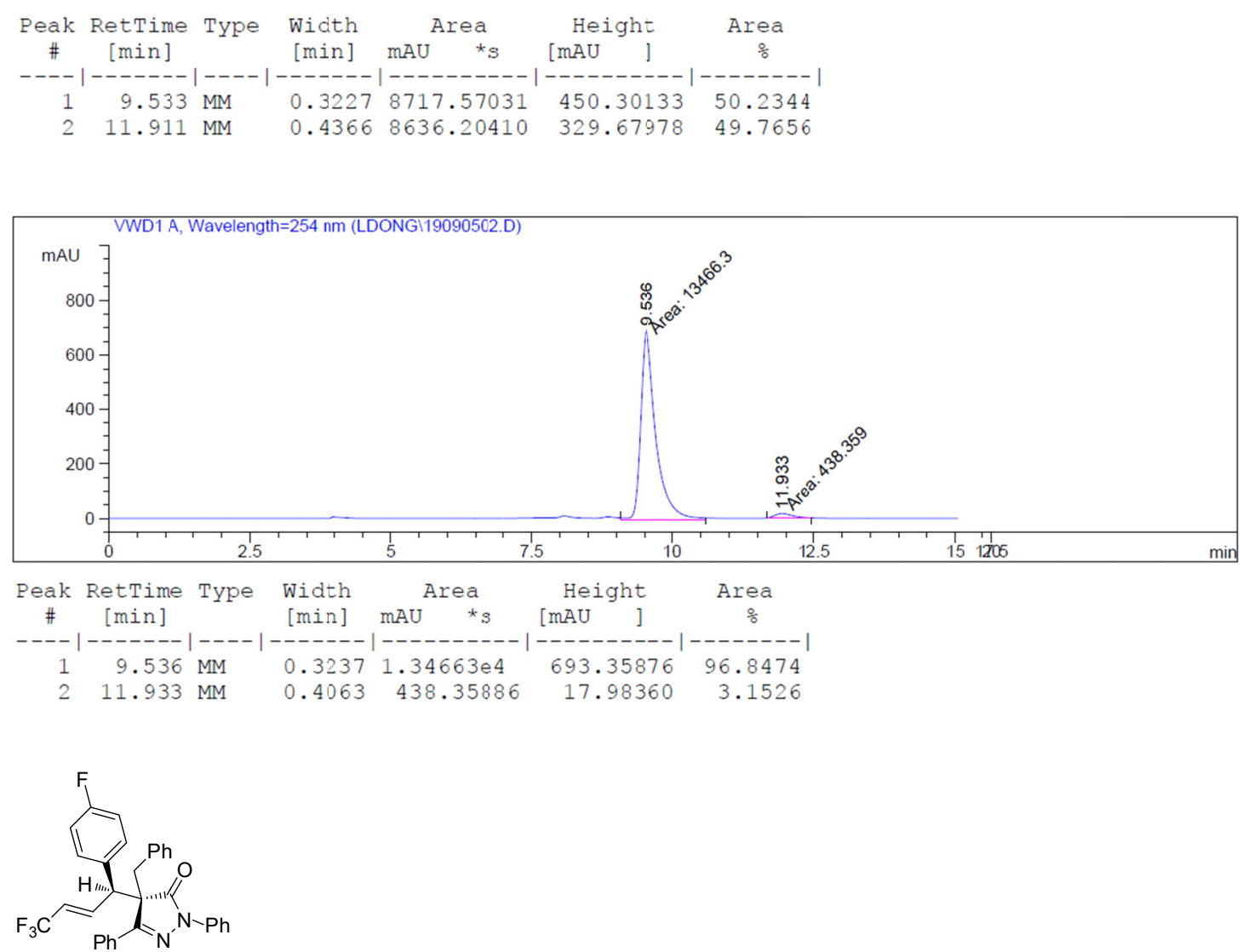

$4 \mathbf{i}$

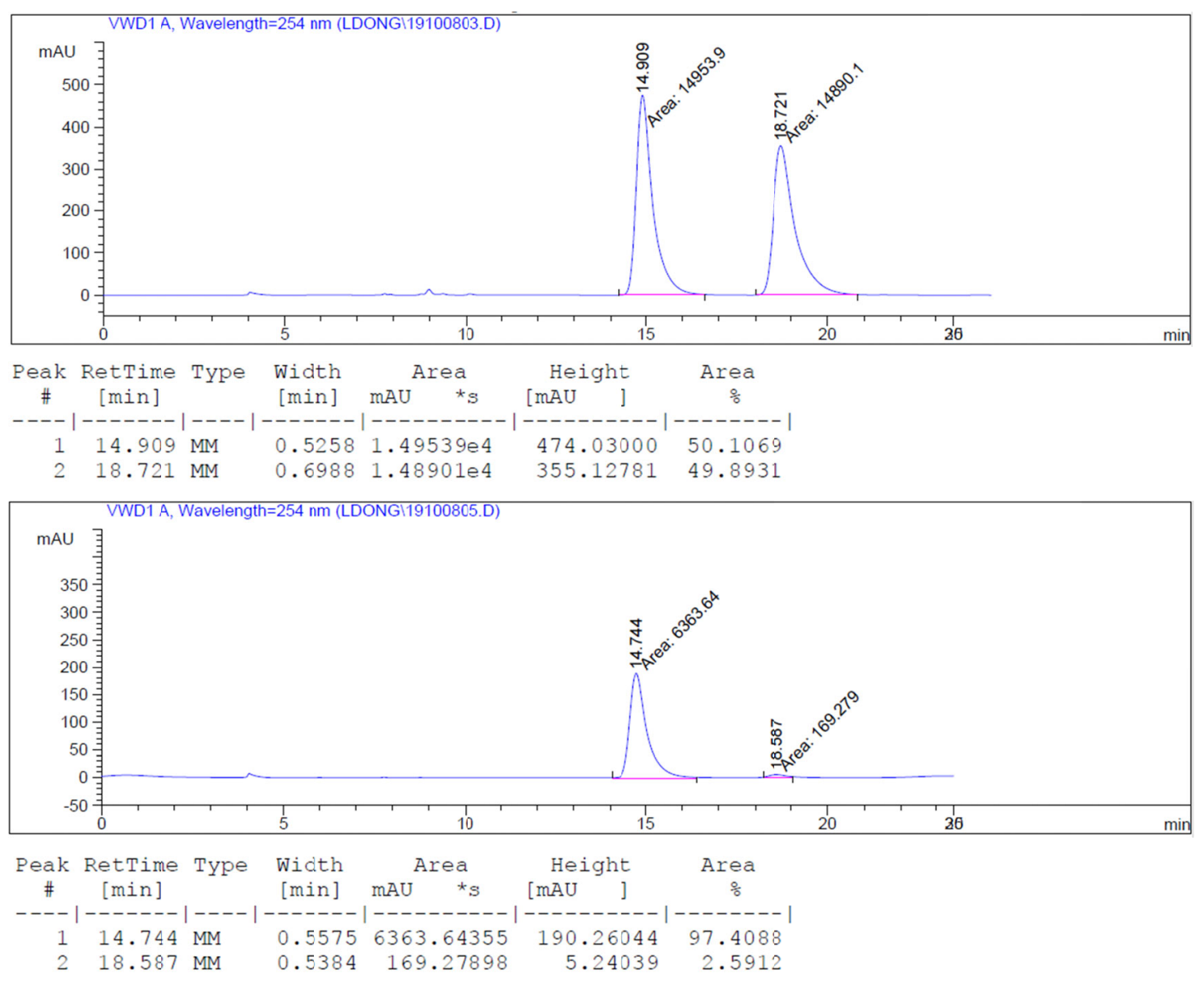

S108 


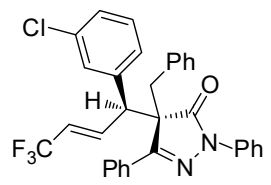

4j
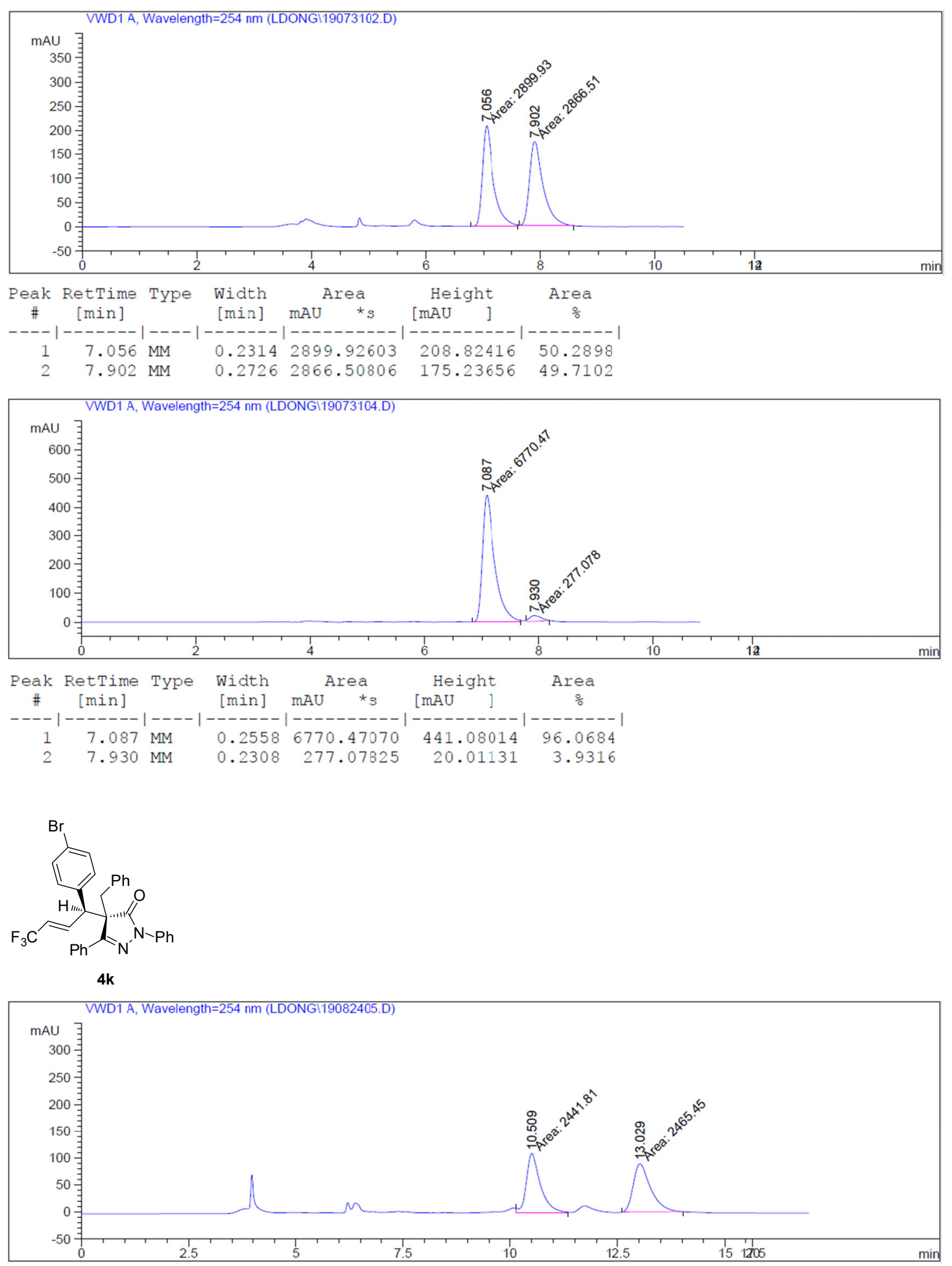

S109 

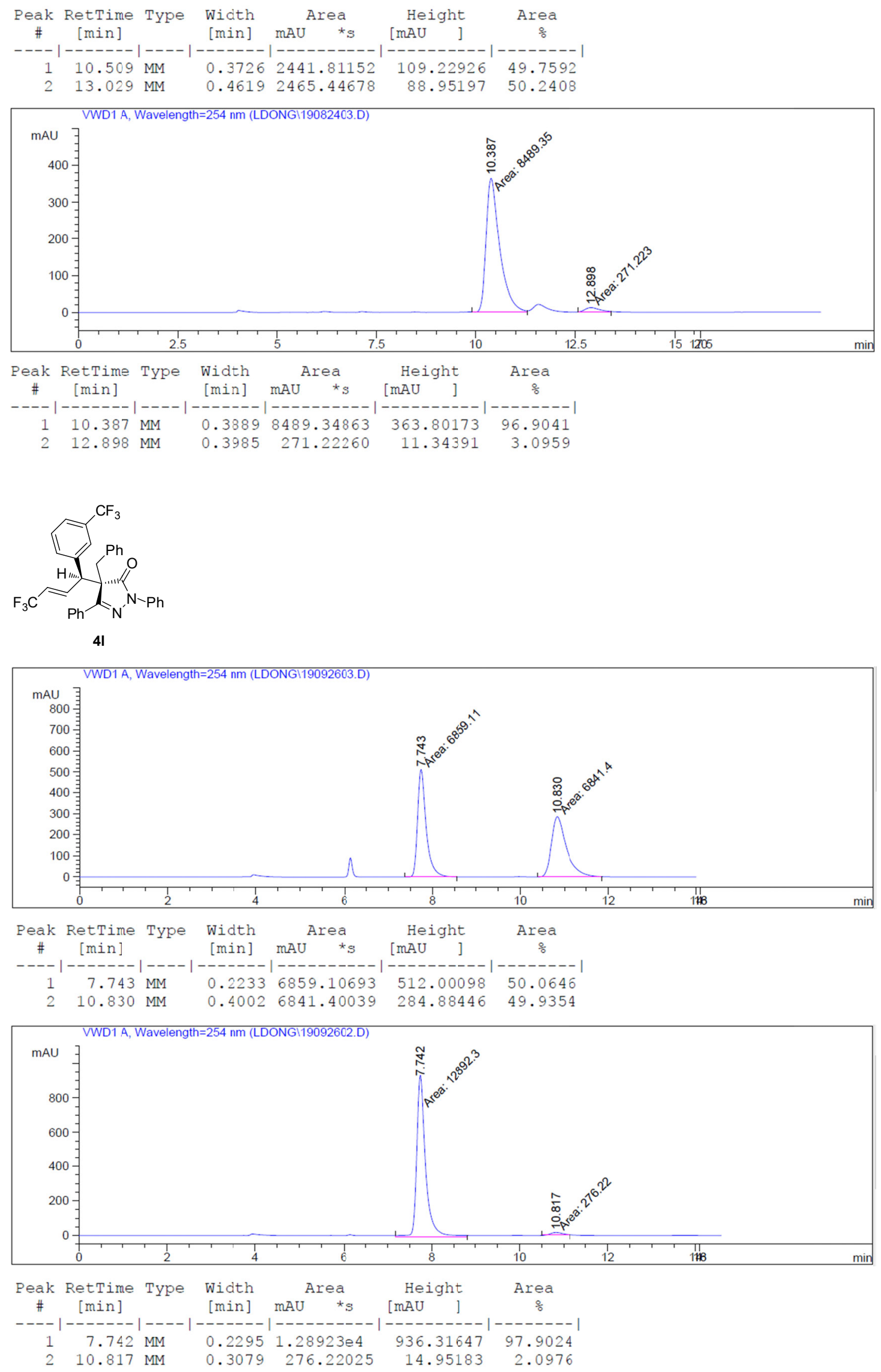

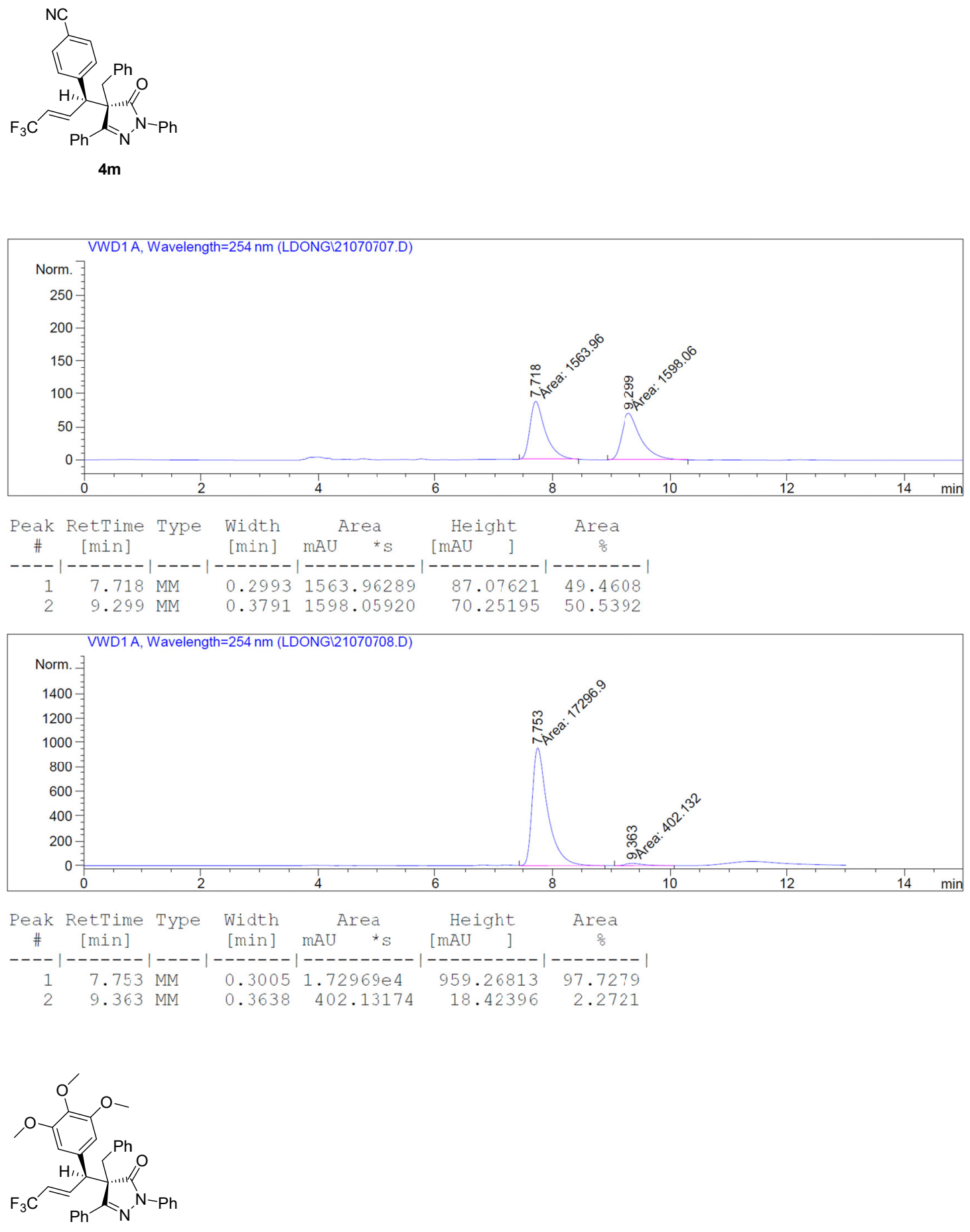

$4 n$

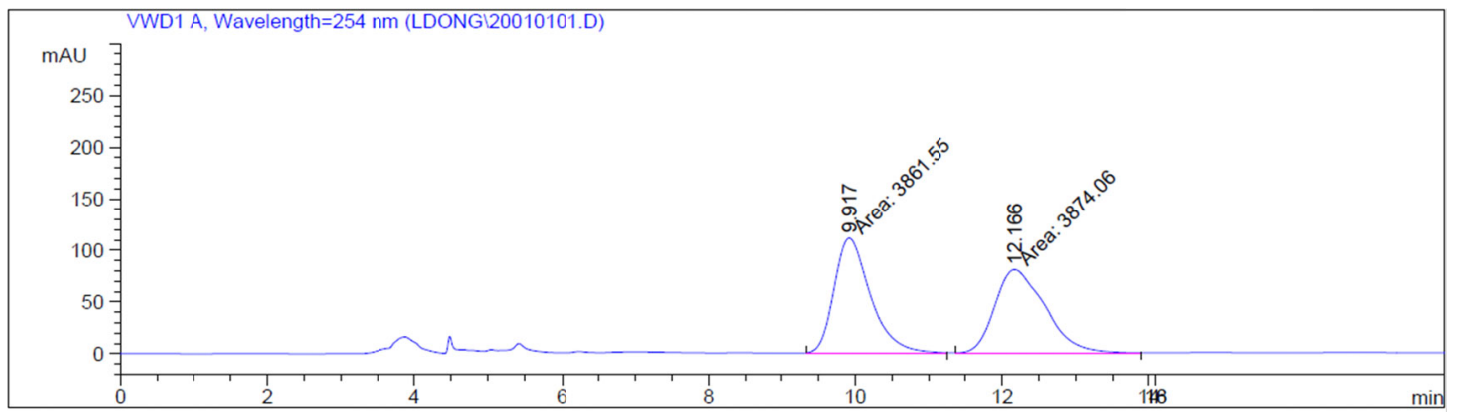



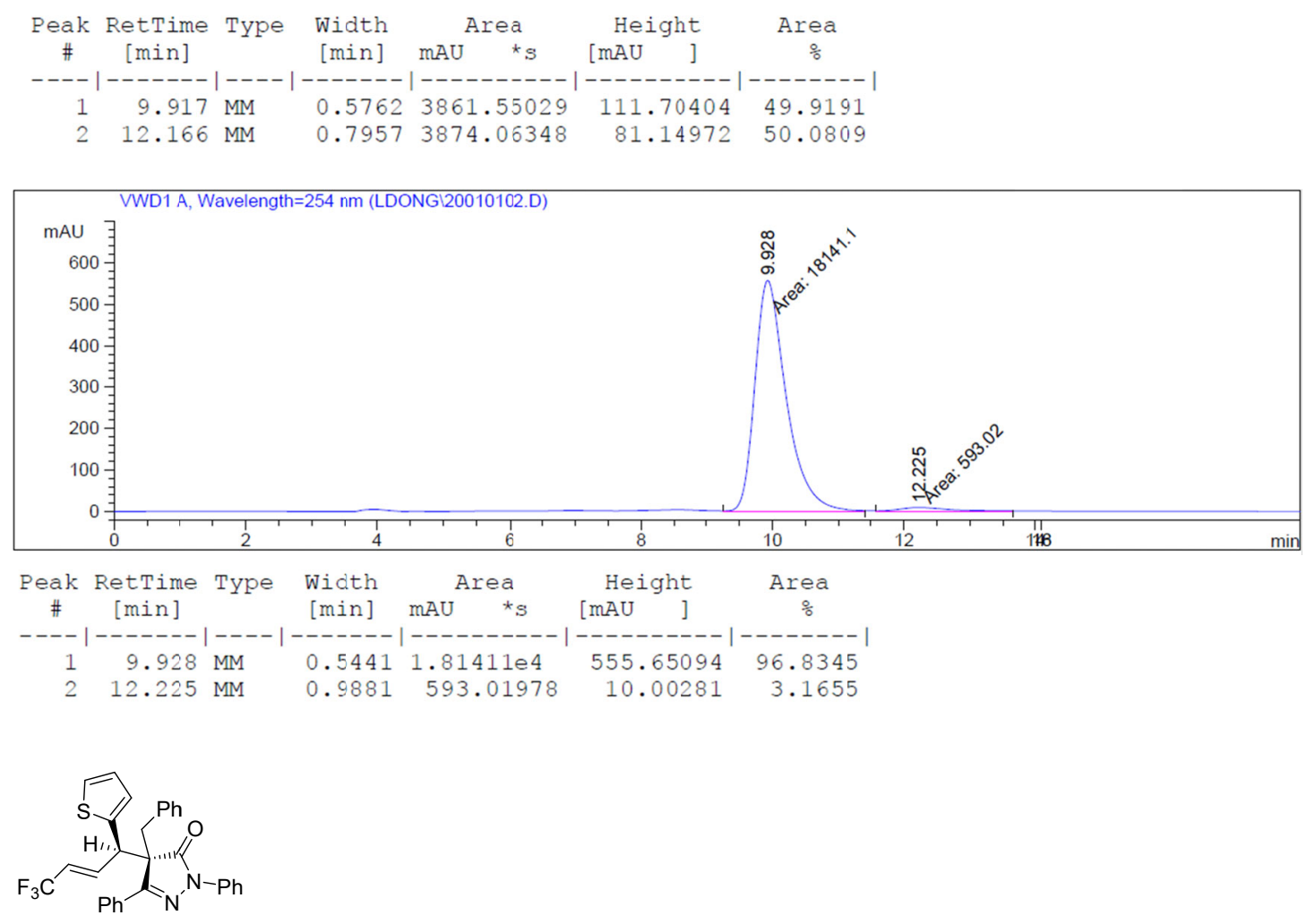

40
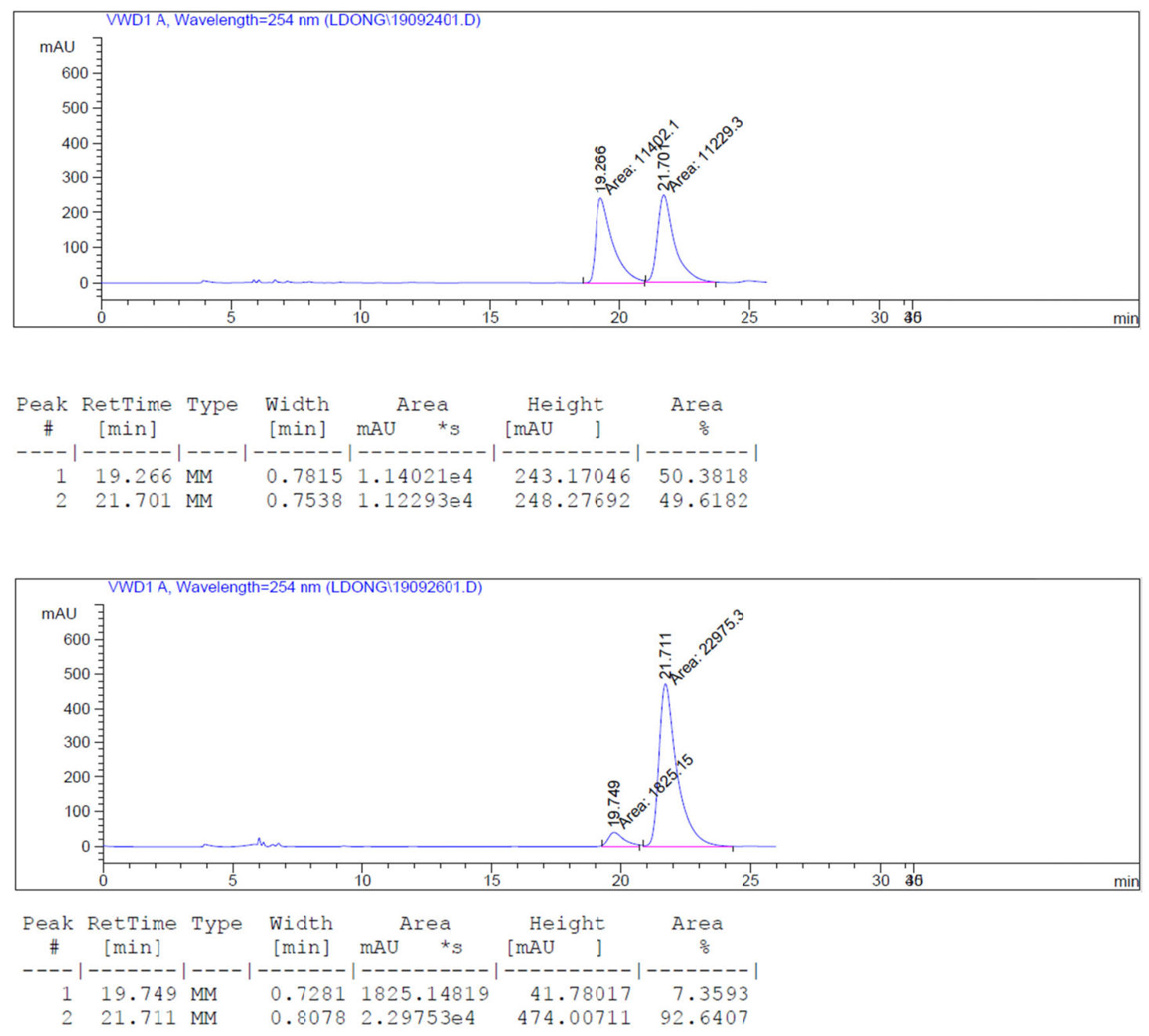

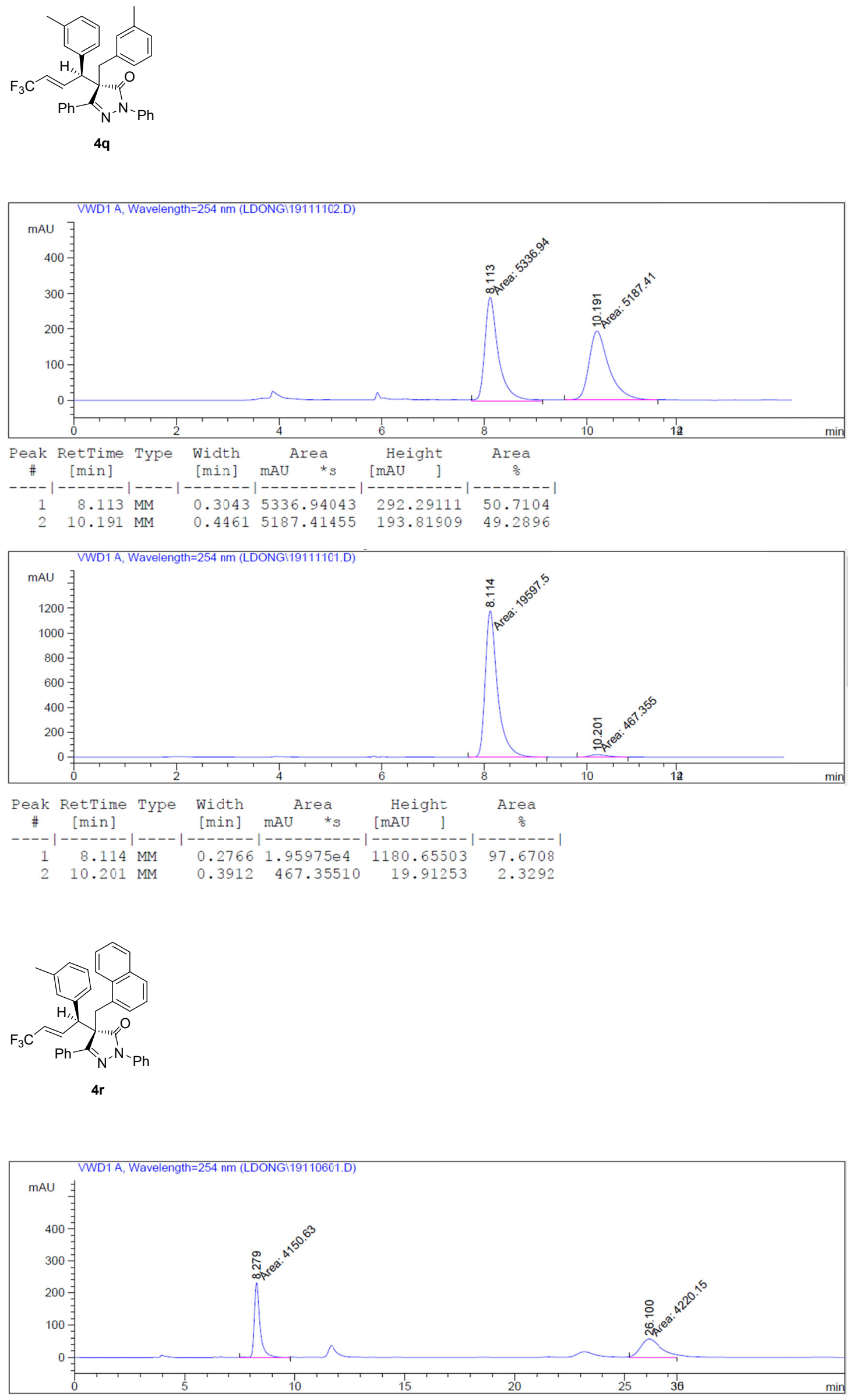

S113 

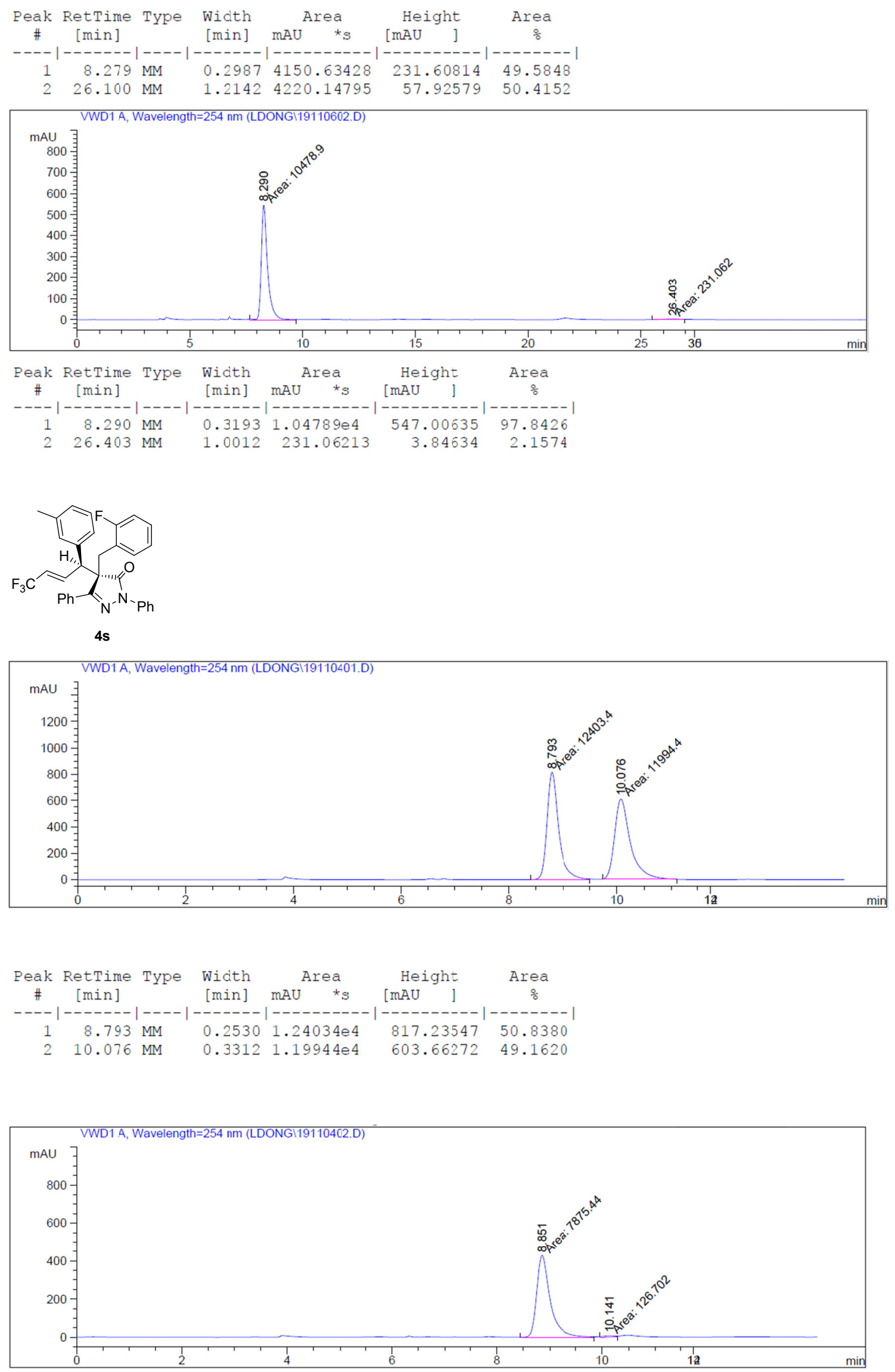

S114 

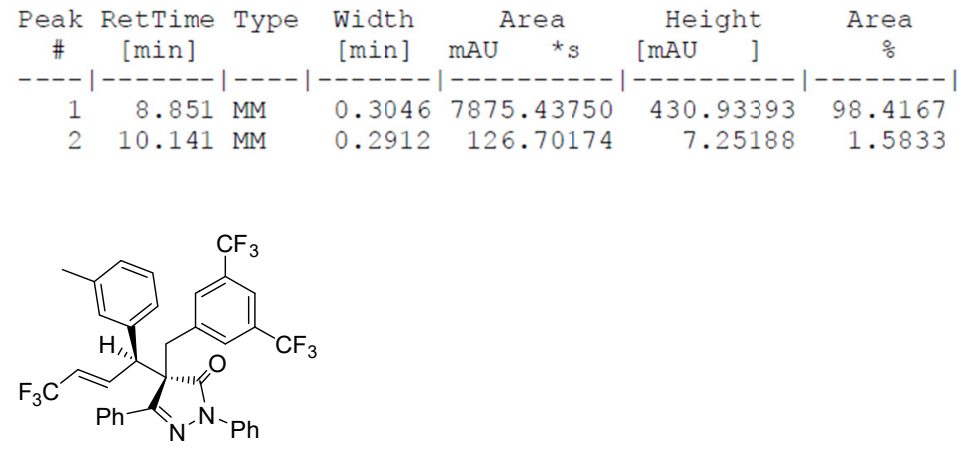

$4 t$
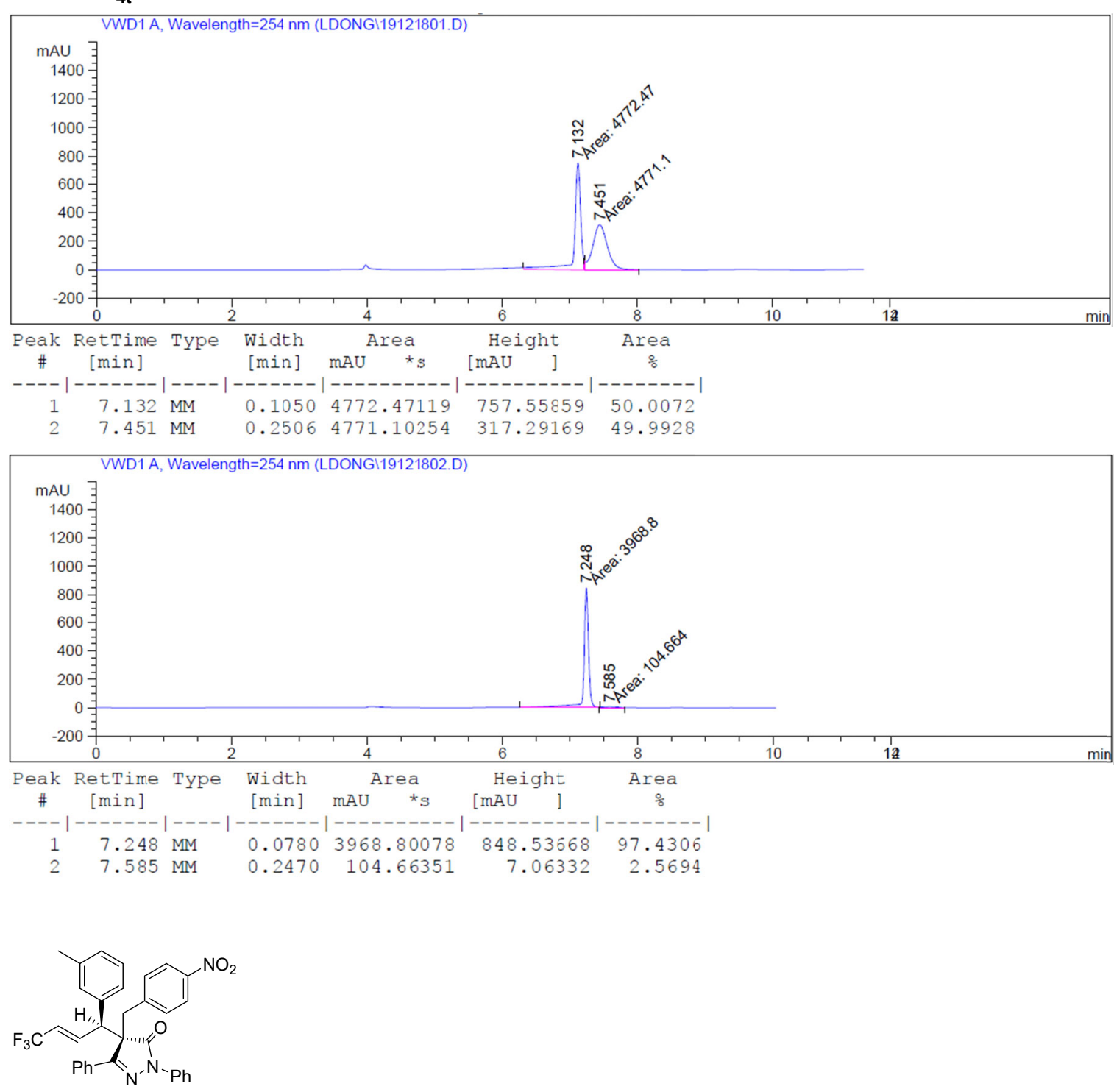

$4 u$ 


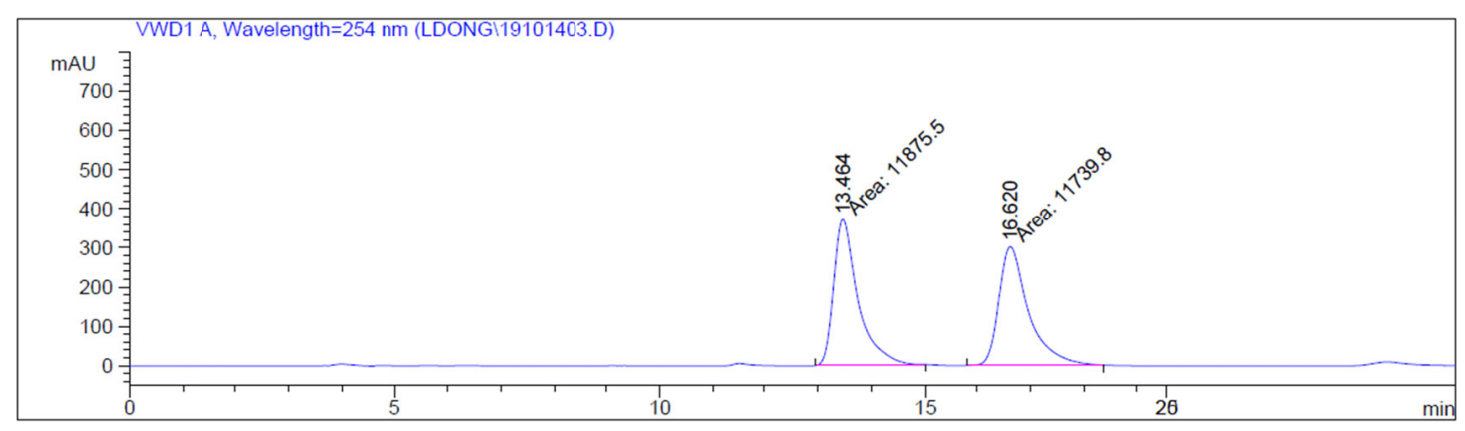

\begin{tabular}{|c|c|c|c|c|c|c|}
\hline Peak & RetTime & Type & Width & Area & Height & Area \\
\hline \# & [min] & & {$[\min ]$} & $\mathrm{mAU} \quad{ }^{*} \mathrm{~s}$ & {$[\mathrm{mAU} \quad]$} & 옹 \\
\hline 1 & 13.464 & MM & 0.5320 & $1.18755 e 4$ & 372.02515 & 50.2872 \\
\hline 2 & 16.620 & MM & 0.6476 & $1.17398 \mathrm{e} 4$ & 302.14951 & 49.7128 \\
\hline
\end{tabular}
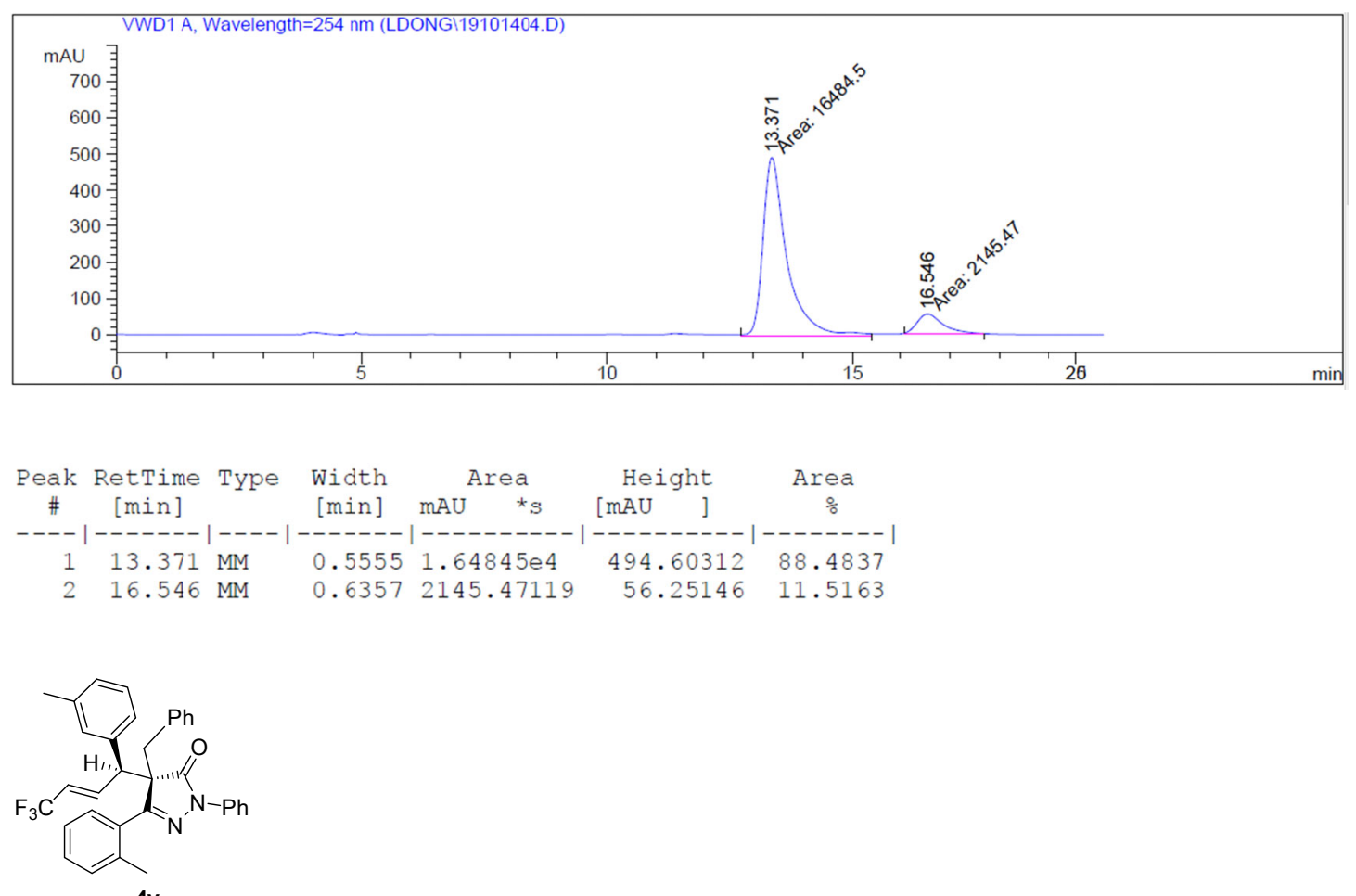

$4 v$
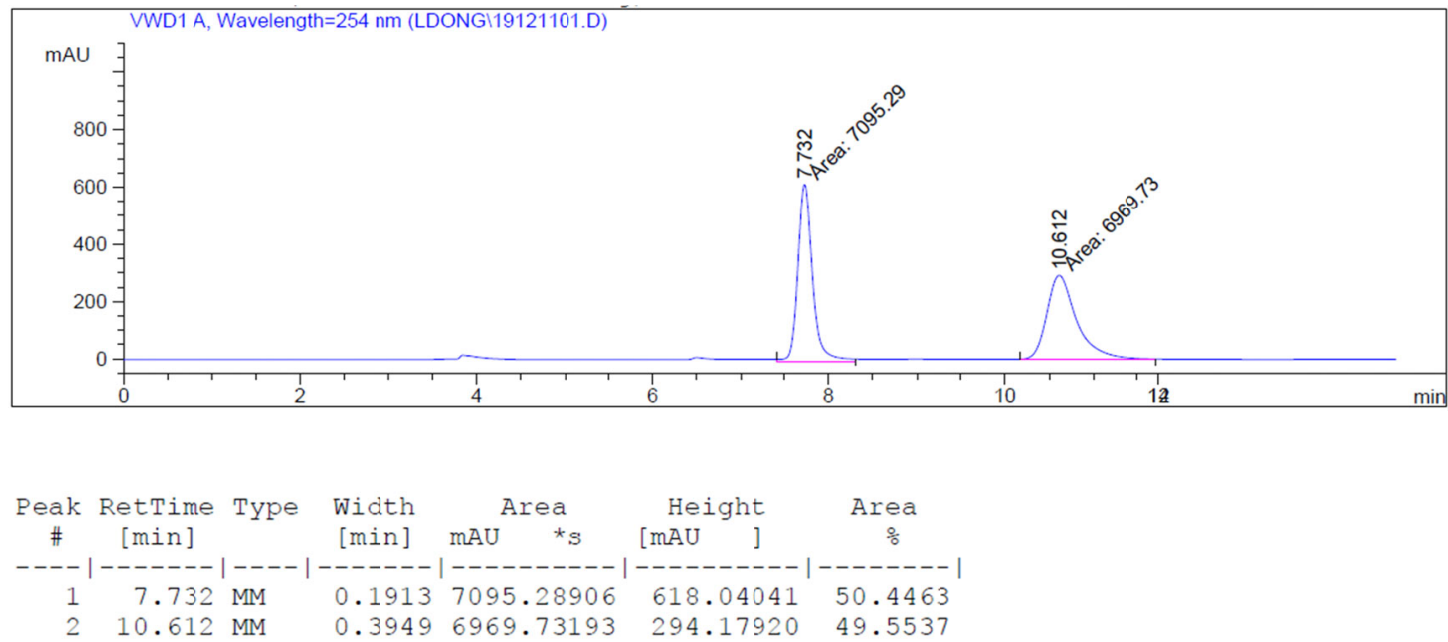

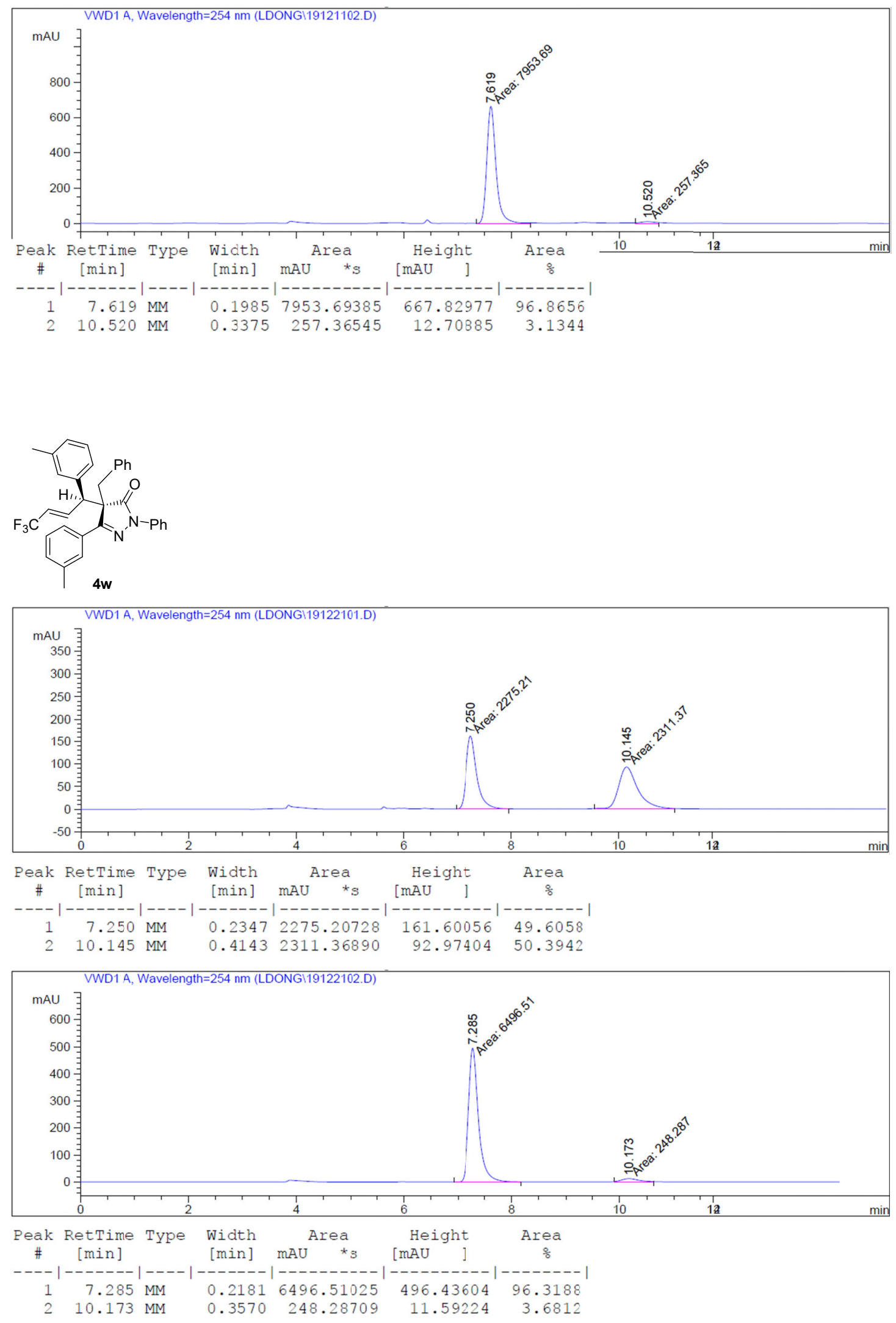


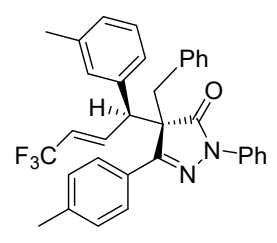

$4 x$
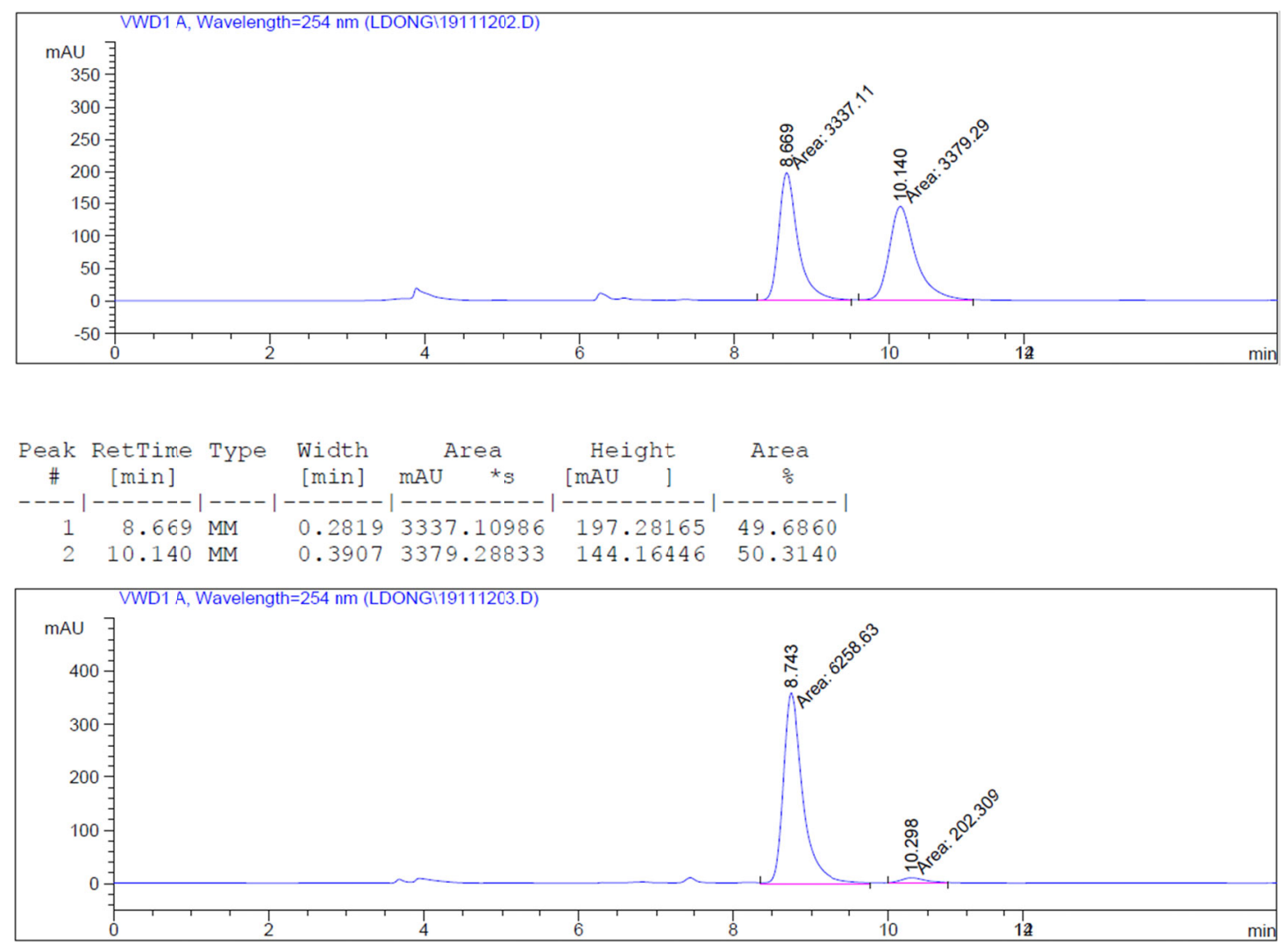

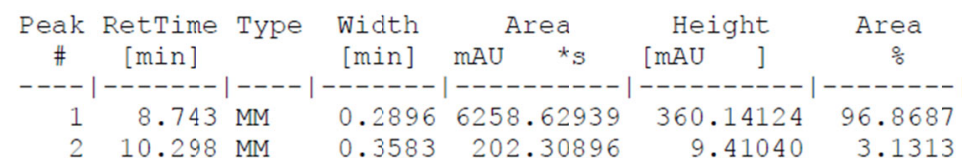

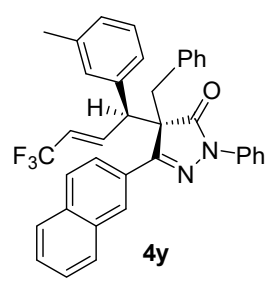



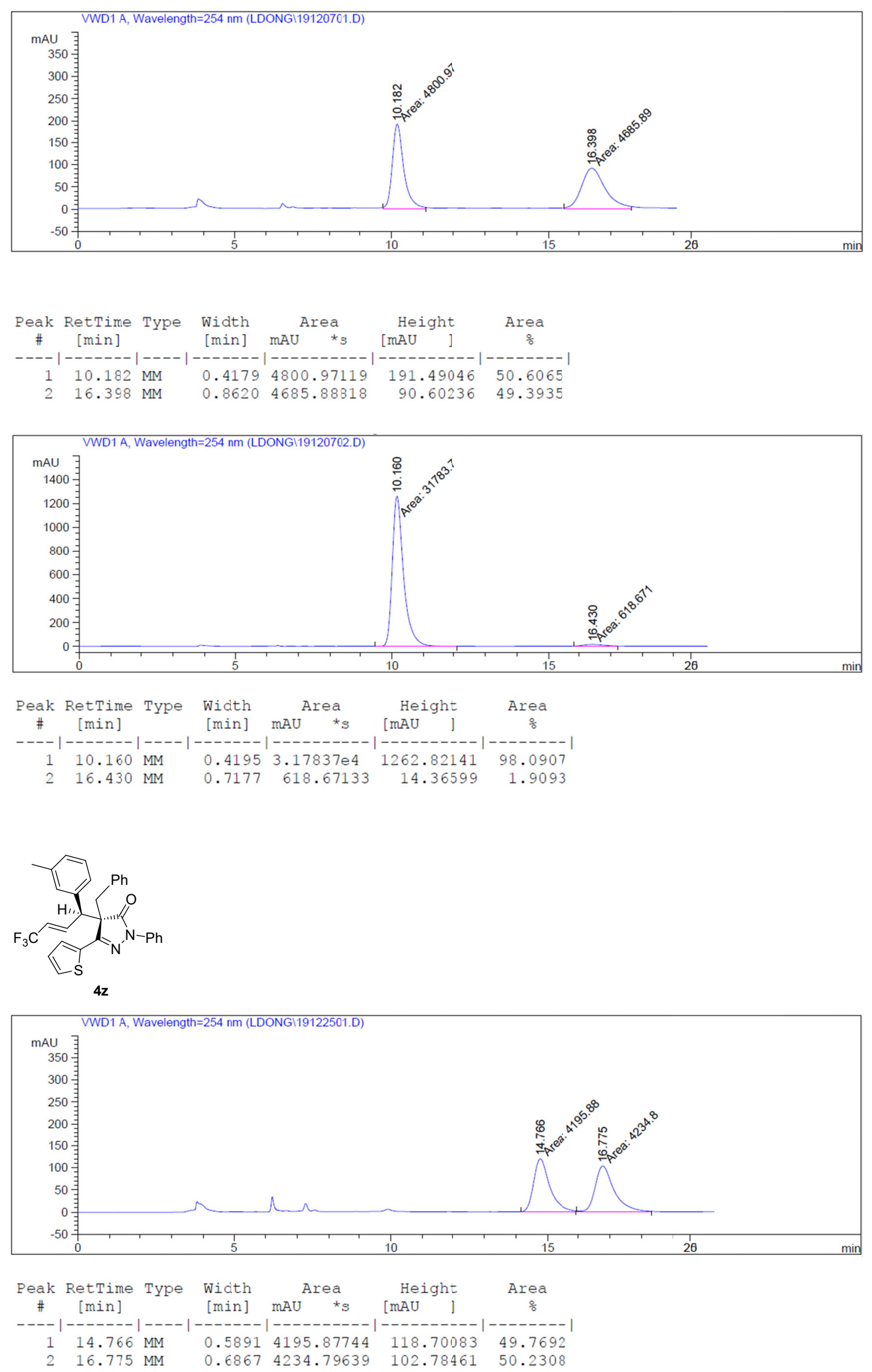

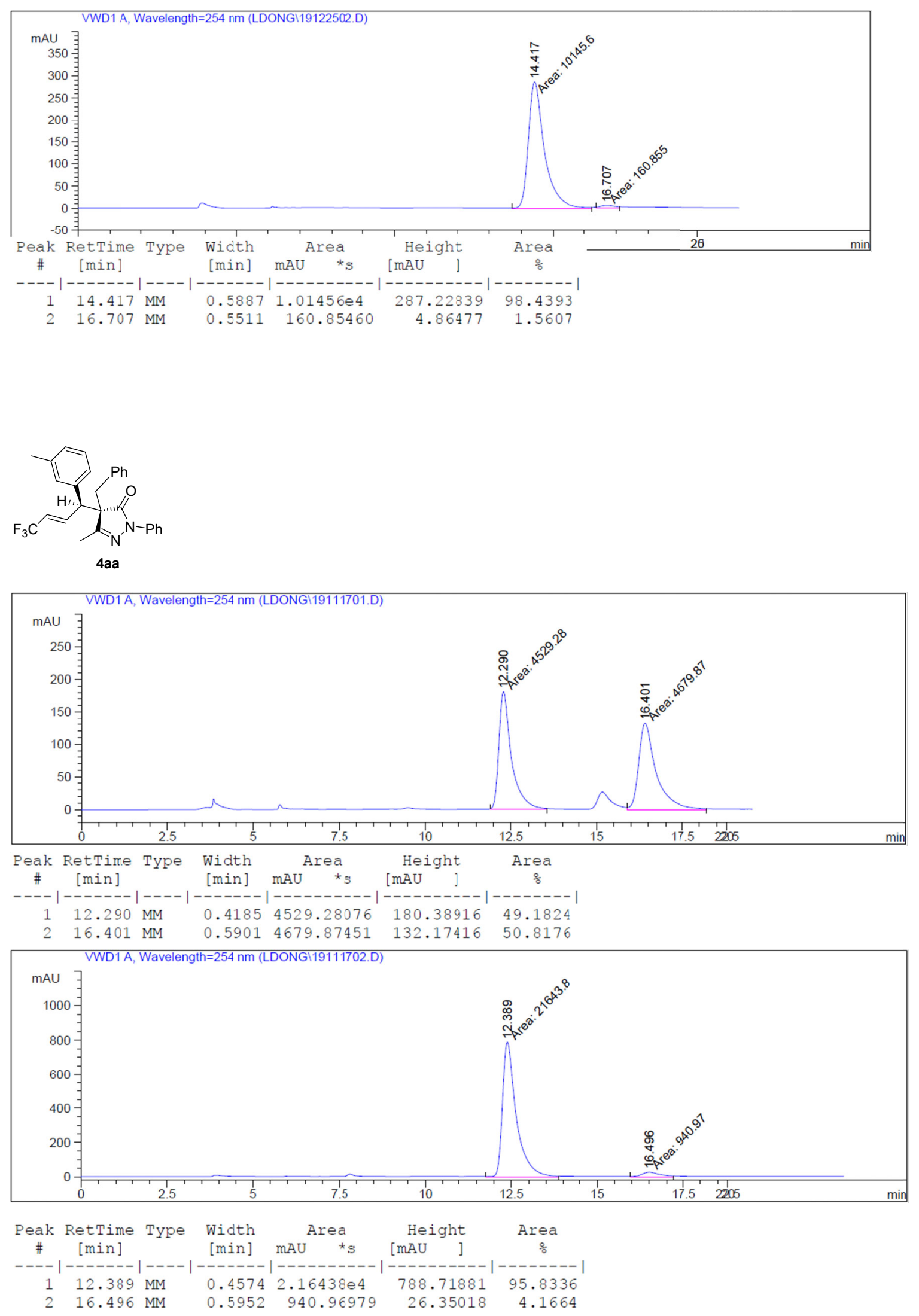

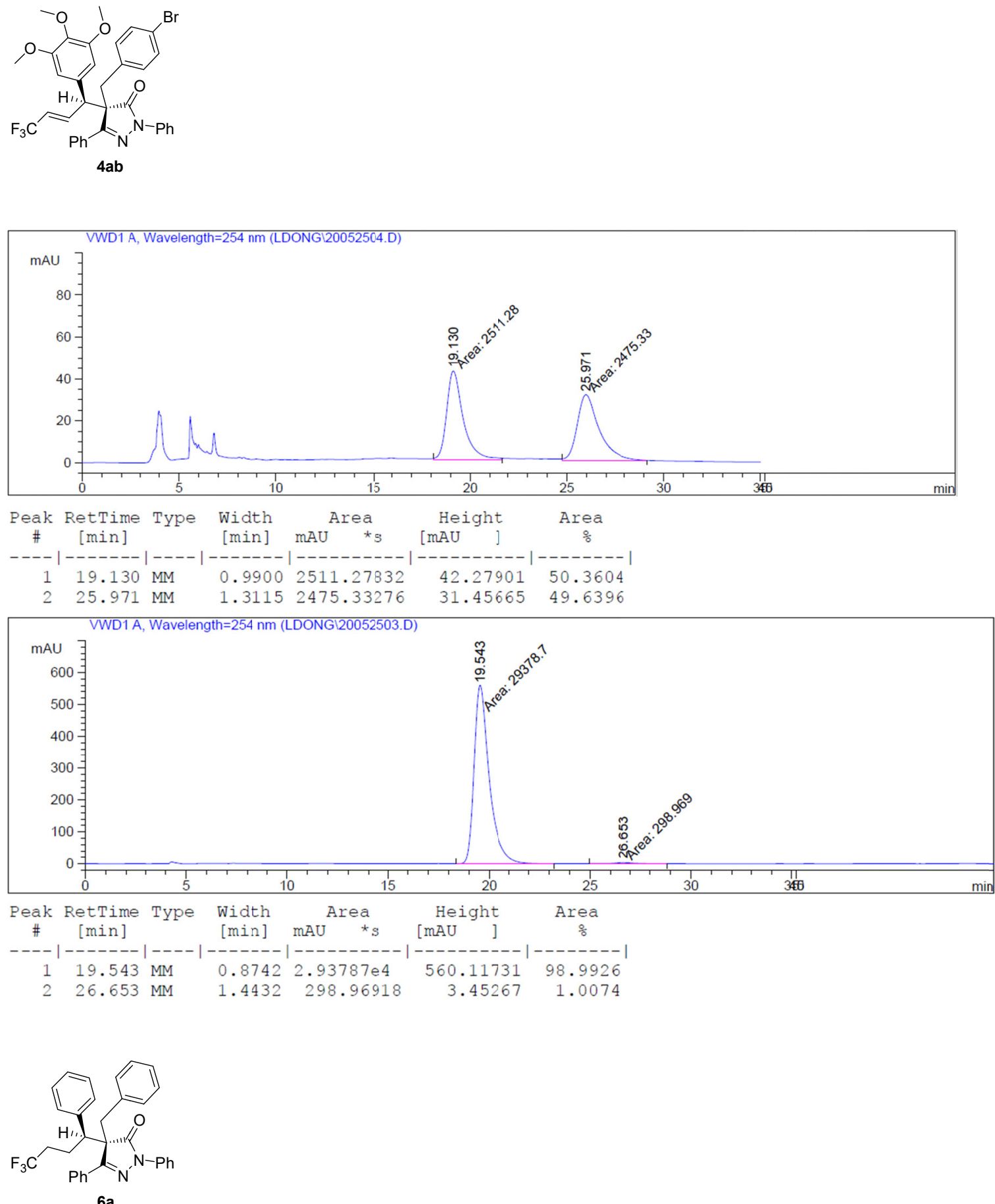

$6 a$

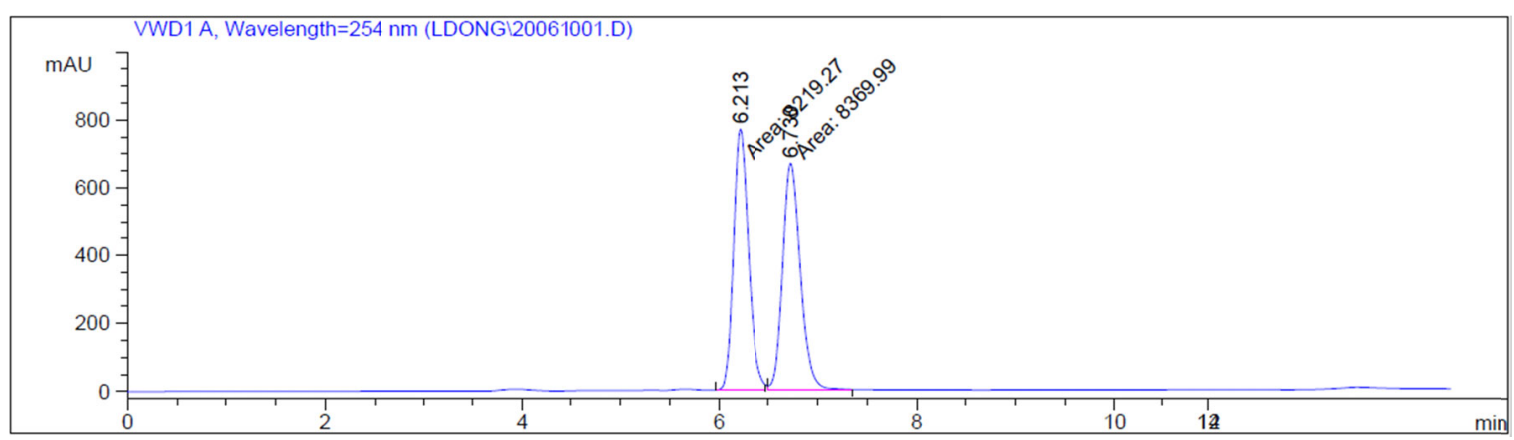



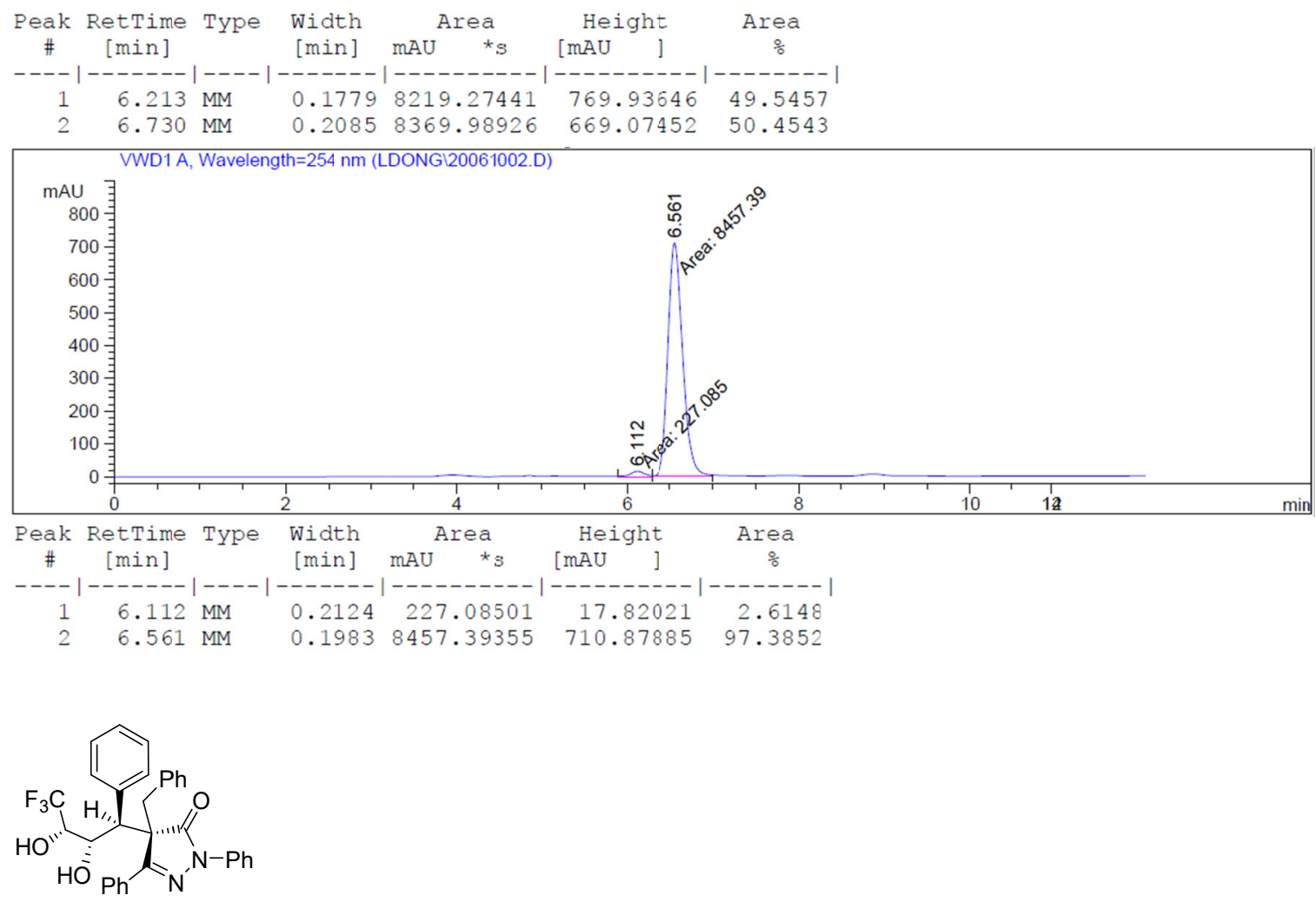

$7 a$
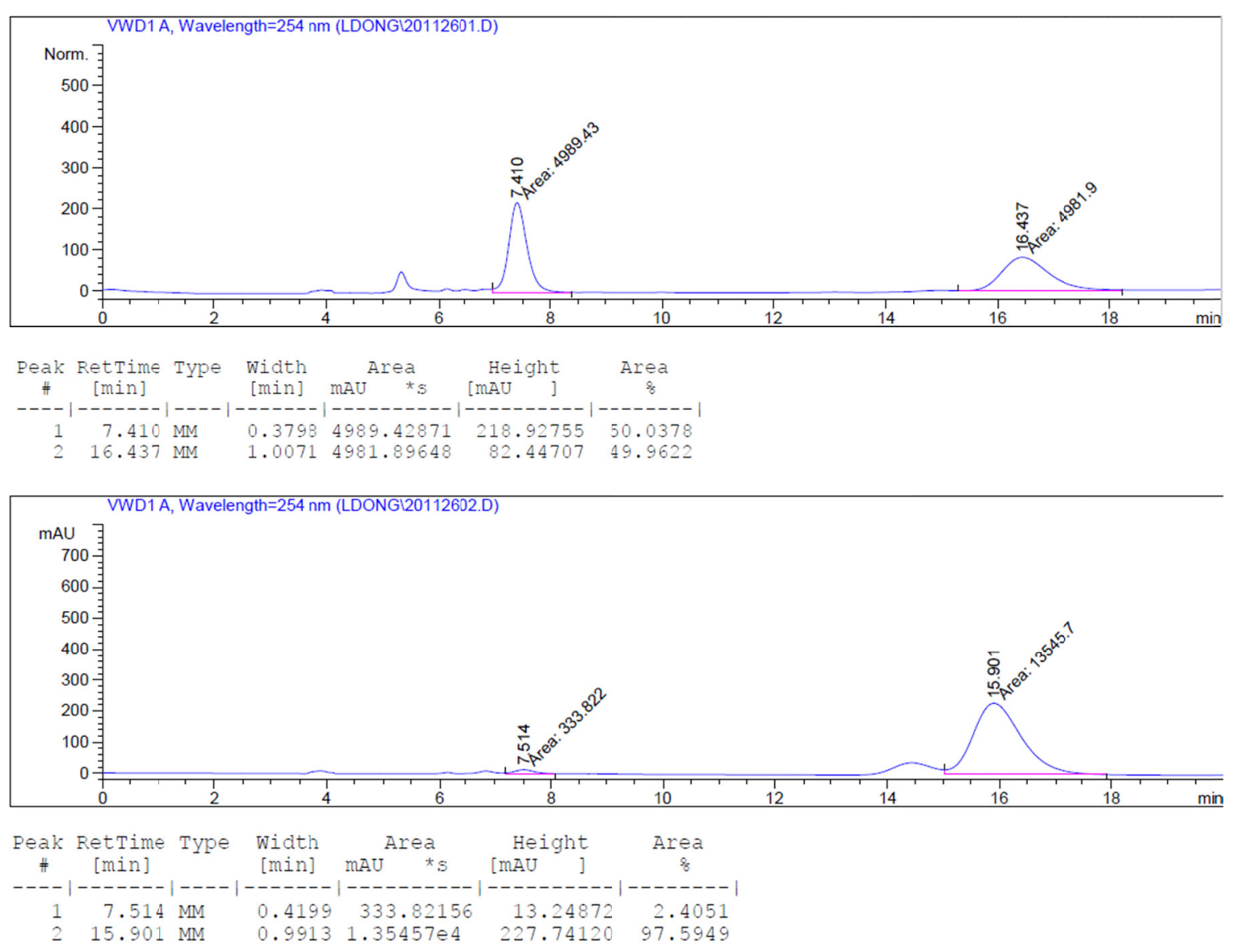